\title{
Estimação dos Parâmetros da máquina síncrona e seu sistema de excitação
}

\author{
Elmer Pablo Tito Cari
}

Dissertação de Mestrado apresentada à Escola de Engenharia de São Carlos, da Universidade de São Paulo, como parte dos requisitos para obtenção do Título de Mestre em Engenharia Elétrica.

ORIENTADOR: Prof. Dr. Luís Fernando Costa Alberto

São Carlos

2005 


\section{Dedicatória}

Com muito amor dedico este trabalho: aos meus pais Julian e Juana, aos meus irmãos e irmãs Hugo, Miguel e Cesar, Beatriz, Angélica, Leonarda e Olga, as minhas sobrinhas e sobrinhos Cyntia, Ana, Sofia e Anthony, aos meus cunhados e cunhadas Marcelino, Raul, Edwin e Aide, e a todos os meus familiares. 


\section{Agradecimentos}

Ao Cordeiro eterno: Cristo Jesus, porque fizeste em mim a tua morada e tornou-se a minha força, meu canto, minha salvação e esperança que nunca findará. Obrigado por mostrar-me o sentido da vida, e desfrutar da riqueza de um país amistoso e dentre outras coisas de escrever esta dissertação.

- Ao Professor Luís Fernando Costa Alberto pela sua orientação, seu excelente exemplo como pessoa e sua amizade durante o presente trabalho.

- Ao Professor Newton Geraldo Bretas por dar-me a oportunidade de fazer o Mestrado, pela sua confiança depositada, sua ajuda na parte final deste trabalho e sobretudo, sua amizade, pela qual estou muito agradecido.

- Aos Professores João Bosco e Rodrigo, pela amizade compartilhada no LACO.

- Aos companheiros do laboratório LACO, aqueles que encontrei ao chegar, aqueles que já foram e ainda aqueles que estão chegando: George, Augusto, Cleito, Lucas, Ivo, Renato, André, Ricardo, Flavio, Fernando, Tais, Eduardo, Lizandra, Ana Paula e Madeleine. A todos vocês, muito obrigado pelos tempos vividos e a amizade que compartilhamos.

- Aos meus companheiros do departamento: Silvio, Elis, Marco, Julia, Rodrigo, Thiago, Luís, Murilo, Odilon, Everthon, Enio, Elcio, Fernanda, Liliane, Anderson, Claudia, Lilian, Glaucia Nilson, Rivail, Carla, entre outros.

- Aos meus amigos da minha ex-república pelos tempos vividos: Basílio, Evandro, Paulo, Luís, Gustavo e João.

- Aos amigos Peruanos, Jose Luís, Enrique, Ivan, Jean, Henry, Cesar, Waldo, Rosamel, Maribel e Roxana, entre outros.

- A Keila e ao Renato amigos que conheci na Unicamp, obrigado pela sua 
ajuda e amizade.

Aos meus amigos da África: Samuel, Mamadú, Paulo e Baptista, irmão na fé, pela amizade compartilhada.

- Ao irmão Mathias e ao Pastor por terem-me mostrado quão doce e maravilhoso é o Senhor.

- Ao Julio por brindar-me sua ajuda como verdadeiro irmão e desejo que possamos crescer juntos nestes tempos de pós-graduação.

- Aos irmãos do projeto Entendendo a Bíblia nas universidades: Melisa, Patrícia, Ana Carolina, Rebeca, Devora, Nívia, Priscila, Elisabeth, Thiago, Timóteo, Diego, Daniel, Lucas, Gesner, Natanael, Rafael, Bruno, Fabiano, Felipe, Diogo, Silas, Vinicius, Henrique, Chin, Mathias, Fernandinho e a todos os que participam este ano.

- A os irmãos da ABU 'Aliança Bíblica Universitária', pela sua amizade.

- Aos irmãos das Igrejas no Perú, no Brasil e no mundo, em especial aos irmãos e irmãs da Igreja em São Carlos, pelas orações e por acima de tudo terem sido minha família, uma única e universal família; ao César e família; Cidinho e família; Zezinho e família; Osório e família; Martins e família; Jurandir e família; Dona Antonieta; Robson e família; Israel e família; Nivaldo e família; Christian e Kelly; João e Família; Cristina e família; Eder e família; Abdalan e família; Daniel e Família; Patracón e Família; Silvio Luporini e família; Leda e Família; André e família; Eduardo e Família; Carlos Iara e Heitor; Mauriza, Álvaro, Ellen, Luciana, Caio, Daniel, Eder, Maurílio e Emerson. Obrigado irmãos, pois aprendi a desfrutar o amor do Senhor cada dia da minha vida.

- Aos irmãos do CEAPE 'Centro de aperfeiçoamento para pregação do Evangelho' pelos momentos compartilhados no ano que passou.

- Aos amigos da Guarda Universitária do PCASC, em especial aos irmãos na fé: Pedro, Gilmar, Marcos e João, o meu mais fraterno 'Amém'.

- À Fundação de Amparo a Pesquisa, FAPESP, pela bolsa de estudos concedida.

- E muito em especial, ao cidadão Brasileiro, que cada dia tem-me mostrado 
a sua férrea vontade de ser amistoso e hospitaleiro, e para quem peço a Deus que derrame todas as suas bênçãos. 


\section{Resumo}

Cari, Elmer Pablo Tito,(2005). Estimação dos Parâmetros da máquina síncrona e seu sistema de excitação. Dissertação (Mestrado) - Escola de Engenharia de São Carlos, Universidade de São Paulo, São Carlos, 2005.

Neste trabalho propõe-se uma metodologia de estimação de parâmetros da máquina síncrona e do sistema de excitação baseada na técnica de sensibilidade de trajetória e sincronização de sistemas. Diversas metodologias de estimação de parâmetros foram propostas na literatura, cada uma com suas vantagens e desvantagens. Entretanto, poucas são adequadas para a estimação de parâmetros com o gerador em operação ('on-line'). A metodologia de sensibilidade de trajetória foi escolhida por permitir uma completa representação da máquina síncrona e do sistema de excitação incluindo as não linearidades como, por exemplo, os limites do sistema de excitação e a saturação do gerador. Foram propostas algumas alternativas para esta técnica visando uma futura aplicação 'on-line'. Dentre as propostas destaca-se o uso da teoria de sincronização no processo de estimação de parâmetros. A metodologia desenvolvida é aplicada para identificar os parâmetros do gerador síncrono (modelo clássico, modelo de um eixo, e modelo de dois eixos). Também são relatados alguns dos problemas encontrados no momento de estimar vários parâmetros da máquina síncrona simultaneamente. A metodologia também é aplicada com sucesso para estimar os parâmetros do sistema de excitação (tipo ST1A e DC1). Por fim, analisa-se a influência dos parâmetros estimados na estabilidade transitória. Para tal objetivo são realizados testes de estabilidade em um pequeno sistema de potência sujeito a incertezas nos parâmetros.

Palavras-Chave: Sensibilidade de trajetória, estimação de parâmetros, máquina síncrona, sistema de excitação. 


\section{Abstract}

Cari, Elmer Pablo Tito(2005). Estimation of the parameter of synchronous machine and its excitation system. Dissertation (Master study)-Escola de Engenharia de São Carlos, Universidade de São Paulo, São Carlos, 2005.

In this work a methodology to estimate parameters of synchronous machines and their excitation system based on trajectory sensitivity technique and system synchronization is proposed. Many methodologies for parameter estimation have been proposed in the literature, each one with advantages and desvantagens. However, only some of them are adequate to estimate parameters with generator in operation ('on-line'). The trajectory sensitivity methodology has been chosen as it allows a complete representation of generator and excitation system including non-linearities as, for instance, the limits of excitation system and saturation of generator. Some alternatives were proposed for this technique regarding a future 'on-line' application. Among them, the use of synchronization theories in parameter estimation process is highlighted. The methodology developed is used to estimate the parameters of generators (classical model, one-axis model and two axis-model). Some problems to simultaneously estimate several parameters of a synchronous machine are also presented. The methodology is successfully used to estimate the parameters of the excitation system (type ST1A and DC1). Finally, the influence of the estimated parameters on transient stability is analyzed. For this aim, stability tests were performed in a small power system with uncertain parameters.

Key-words: Trajectory sensitivity, parameter estimation, synchronous machine, excitation system. 


\section{Lista de Figuras}

$1.1 \quad$ Estrutura Metodológica da dissertação $\ldots$. . . . . . . . . . . . . . 6

2.1 Corrente de curto circuito na fase a $I_{a}$, e no enrolamento de campo

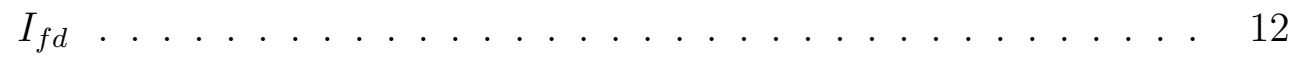

2.2 Resposta da corrente da fase 'a' após um curto circuito no estator. 13

2.3 Componentes da corrente de curto circuito em escala semi-logarítmica 14

2.4 Determinação das constantes de tempo da máquina síncrona . . . 15

2.5 Diagrama fasorial no teste de rejeição de carga. $\ldots$. . . . . . . . . 17

2.6 Tensão terminal da máquina síncrona após a rejeição de carga no eixo direto . . . . . . . . . . . . . . . . . . . . . . . . . . . 17

2.7 Tensão terminal da máquina síncrona após a rejeição de carga no eixo em quadratura. . . . . . . . . . . . . . . . . . . 18

2.8 Métodologia com tese de inclução de perturbações PRBS no AVR. 19

2.9 Representação dos terminais no eixo direto e no eixo em quadratura baseado na equação refequa:freqfluxdq. . . . . . . . . . . . . . . . 22

2.10 Posição do rotor para o teste em eixo direto. . . . . . . . . . . . . 23

2.11 Teste para a medida de $Z_{d} . \ldots \ldots \ldots$. . . . . . . . . . 23

2.12 Impedância em eixo direto de $Z_{d}$ em função da freqüência. . . . . 24

2.13 Teste para a medida de $\mathrm{Z}(\mathrm{q})$. . . . . . . . . . . . . . 25

2.14 Comparação de algumas metodologias de estimação de parâmetros. 29

3.1 Saída e funções de sensibilidade para o exemplo 1. . . . . . . . . . 33

3.2 Metodologia de sensibilidade da trajetória para determinação de parâmetros. . . . . . . . . . . . . . 36 
3.3 Comparação da saída do 'modelo matemático' e do 'sistema real'. 37

3.4 Diagrama esquemático da metodologia de Sensibilidade da trajetória. 40

3.5 Representação física do sistema massa mola. . . . . . . . . . . . . . 41

3.6 Diagrama esquemático para a estimação dos para o sistema massamola. . . . . . . . . . . . . . . . . . . . . 42

3.7 Saída inicial e final no teste de determinação de 1 parâmetro. . . . 43

3.8 Entradas e saídas para estimação de 'k' e 'm' simultaneamente. . 45

3.9 Saída do 'modelo matemático' e 'sistema real' antes e após a estimação dos dois parâmetros no sistema massa-mola. ..... 47

4.1 Processo de Estimação de parâmetros. . . . . . . . . . . . . . . 50

4.2 Continuidade com relação aos parâmetros. . . . . . . . . . . . . . 51

4.3 Estimação de parâmetros por sincronização. . . . . . . . . . . . . 53

4.4 Estimação com a técnica de sensibilidade de trajetória. . . . . . . 54

4.5 Estimação com a técnica de sensibilidade de trajetória mais sincronização. . . . . . . . . . . . . . . . . . . . . . . 54

5.1 Máquina síncrona de dois pólos. . . . . . . . . . . . . . . 59

5.2 Equivalente da máquina síncrona na referência abc. . . . . . . . . 60

5.3 Circuito Equivalente da máquina Síncrona (referência 0dq). . . . . 62

5.4 Circuito equivalente do eixo d. . . . . . . . . . . . . 63

5.5 Circuito equivalente do eixo q. . . . . . . . . . . . . 63

5.6 Modelo Equivalente (1) da Máquina Síncrona. . . . . . . . . . . . 64

5.7 Modelo Equivalente (2) da Máquina Síncrona. . . . . . . . . . . . 64

5.8 Modelo Equivalente (3) da Máquina Síncrona. . . . . . . . . . . . 64

5.9 Máquina Síncrona de dois pólos. . . . . . . . . . . . . . . 65

5.10 Sistema de excitação ST1A. . . . . . . . . . . . . . 73

5.11 Sistema de excitação DC1 . . . . . . . . . . . . . . . . 75

6.1 Processo de estimação de parâmetros. . . . . . . . . . . . . . . . . 79

6.2 Condições de Operação no processo de estimação. . . . . . . . . . 79

6.3 Modelo do sistema de potência . . . . . . . . . . . . . . . . 80 
6.4 Modelo clássico. . . . . . . . . . . . . . . . . . . . . . . . . 81

6.5 Transformação das equações originais do modelo clássico. . . . . . 83

6.6 Diagrama esquemático da aplicação da metodologia de estimação de parâmetros para o modelo clássico. . . . . . . . . . . . . . 84

6.7 Comparação da potência elétrica antes e depois a estimação dos parâmetro para o 'modelo clássico' . . . . . . . . . . . . . . 85

6.8 Velocidade angular antes e depois da estimação dos parâmetros para o 'modelo clássico'. . . . . . . . . . . . . . . . . . . 85

6.9 Sistema de potência reduzido aos barramentos terminais da máquina em falta e pós-falta. . . . . . . . . . . . . . . 87

6.10 Variáveis de entrada e saída para o modelo de 'um eixo'. . . . . . 88

6.11 Ângulo do rotor, antes e depois da estimação. . . . . . . . . . . . 90

6.12 Velocidade angular antes e depois da estimação dos parâmetros para o modelo de um eixo . . . . . . . . . . . . . . . . . . . . . . 90

6.13 Sensibilidades das saídas 1 e 2 com relação aos parâmetros. . . . . 91

6.14 Sensibilidades das saídas 1 e 2 respeito aos parâmetros, caso de

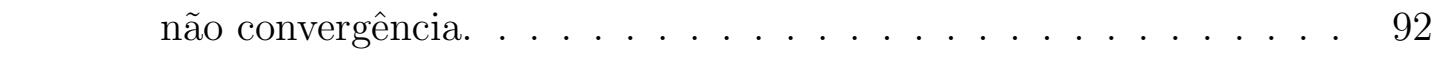

6.15 ângulo(ang), velocidade $(w)$, e $E_{q}^{\prime}$ antes da estimação dos parâmetros. 93

6.16 ângulo(ang), velocidade $(w)$, e $E_{q}^{\prime}$ ao final da $1^{r a}$ fase. . . . . . . . 94

6.17 ângulo(ang), velocidade $(w)$, e $E_{q}^{\prime}$ ao final da $2^{d a}$ fase. . . . . . . . 94

6.18 Sistema de potência para o a estimação de parâmetros do modelo de dois eixos . . . . . . . . . . . . . . . . . . . . . . . . . . 95

6.19 Sistema de referência da máquina e da rede externa . . . . . . . . 96

6.20 Sistema de potência reduzido nos terminais da máquina em falta e pós falta . . . . . . . . . . . . . . . . . . . . . 97

6.21 Determinação da condição inicial para o calculo da função de sensibilidade em relação a $E_{d o}^{\prime}$. . . . . . . . . . . . . . . . . . . 103

6.22 Diagrama esquemático para o teste 1 com $I_{d}$ e $I_{q}$ como entradas . 104

6.23 Velocidade angular antes e depois da estimação dos parâmetros do eixo d no teste $n^{\circ} 1 \ldots \ldots \ldots$. . . . . . . . . . . . 105 
6.24 Velocidade angular $\mathrm{w}$ antes e depois da estimação dos parâmetros do eixo em quadratura no teste $n^{\circ} 1 \ldots$. . . . . . . . . . 105

6.25 Funções de sensibilidade da saída 'w' em relação aos parâmetros para a primeira iteração. . . . . . . . . . . . . . . . . . . . 106

6.26 Velocidade angular antes e depois da estimação dos parâmetros com $x_{d}^{\prime}$ fixo . . . . . . . . . . . . . . . . . 108

6.27 Diagrama esquemático de entradas e saídas no teste $\mathrm{n}^{\circ} 2$. . . . 110

6.28 Diagrama esquemático considerando duas saídas . . . . . . . . . . 112

6.29 Diagrama esquemático considerando duas saídas . . . . . . . . . . 114

6.30 Ângulo do rotor antes e depois do processo de estimação dos parâmetros no teste $n^{\circ} 4 \ldots \ldots \ldots \ldots$

6.31 Velocidade do rotor antes e depois a estimação dos parâmetros no teste $n^{\circ} 4 \ldots \ldots \ldots \ldots \ldots$

6.32 Diagrama esquemático para a estimação de H e D . . . . . . . . . 122

6.33 Diagrama esquemático para estimação dos parâmetros do sistema excitação ST1A. . . . . . . . . . . . . . . . . . . . . . 124

$6.34 E_{f d}$ antes e depois a estimação dos parâmetros . . . . . . . . . . . 124

6.35 Diagrama esquemático para estimação dos parâmetros do sistema de excitação DC1. . . . . . . . . . . . . . . . . . . . . . . 128

6.36 Tensão de campo antes e depois a estimação dos parâmetros . . . 129

A.1 Parte do Sistema Elétrico Sul do Peru. . . . . . . . . . . . . . . . . 144

A.2 Comparação entre a potências de transferência registrada e a simulada computacionalmente. . . . . . . . . . . . . . . . . 145

A.3 Sistema de potência para a aplicação do testes de estabilidade transitória . . . . . . . . . . . . . . . . . . . 146

A.4 Resposta do ângulo do rotor dos geradores com parâmetros verdadeiros . . . . . . . . . . . . . . . . . . . . . 148 


\section{Lista de Tabelas}

3.1 Teste para estimação de um parâmetro do sistema massa mola. . . 44

3.2 Teste para estimação de dois parâmetros do sistema massa-mola. . 47

4.1 Resultados com a técnica de sensibilidade de trajetória tradicional (diverge) . . . . . . . . . . . . . . . . . 55

4.2 Resultados com a técnica de sensibilidade de trajetória tradicional mais sincronização de sistemas (converge). . . . . . . . . . . . . 55

6.1 Estimação dos parâmetros do modelo clássico. . . . . . . . . . . . 86

6.2 Estimação dos parâmetros do modelo de 'um eixo'. . . . . . . . . 91

6.3 Estimação dos parâmetros do modelo de 'um eixo' incluindo $E_{q o}^{\prime}$. .94

6.4 Estimação dos parâmetros de eixo direto no teste $\mathrm{n}^{\circ} 1$ 1. . . . . . . 104

6.5 Estimação dos parâmetros do eixo em quadratura no teste $\mathrm{n}^{\circ}$ 1. . 106

6.6 Estimação de parâmetros da máquina síncrona com mantendo $x_{d}^{\prime}=$ 0.3 no teste $n^{\circ} 1 \ldots \ldots \ldots \ldots$

6.7 Estimação dos parâmetros da máquina síncrona no teste $\mathrm{n}^{\circ} 2$, incluindo $E_{d o}^{\prime}$ e $E_{q o}^{\prime}$. . . . . . . . . . . . . . . . . . 110

6.8 Estimação dos parâmetros da máquina síncrona no teste $\mathrm{n}^{\circ} 3$.

6.9 Estimação de parâmetros da máquina síncrona no teste $\mathrm{n}^{\circ} \quad$ 4. . . . 120

6.10 Estimação dos parâmetros mecânicos da máquina síncrona em cada iteração . . . . . . . . . . . . . . . . . . . 122

6.11 Resumo dos resultados da estimação dos parâmetros da máquina síncrona . . . . . . . . . . . . . . . . . . 122

6.12 Estimação dos parâmetros do sistema de excitação ST1A. . . . . . 124 
6.13 Parâmetros do sistema de excitação DC1. . . . . . . . . . . . . . . 129

A.1 Tensões nas barras terminais dos geradores na condição pré-falta. 147

A.2 Dados das cargas do sistema de potência. . . . . . . . . . . . . . . 147

A.3 Valores dos parâmetros dos geradores. . . . . . . . . . . . . . . . . 147

A.4 Valores dos parâmetros do sistema de excitação. . . . . . . . . . . 147

A.5 Parâmetros do PSS. . . . . . . . . . . . . . . . . . . . . . . . . . . 148

A.6 Influência dos parâmetros do sistema de excitação ST1A do Gerador G1 no cálculo do tempo crítico. . . . . . . . . . . . . . . . . . 149

A.7 Influência dos parâmetros elétricos da máquina síncrona no tempo crítico de abertura. . . . . . . . . . . . . . . . . . . . . . . . . . . 150

A.8 Influência dos parâmetros mecânicos da máquina síncrona no tempo crítico de abertura. . . . . . . . . . . . . . . . . . . . . . . . 151 


\title{
Lista de Abreviaturas e Siglas
}

\author{
AVR Automatic Voltage Regulator \\ AC Alternate Current \\ DC Direct Current \\ IEEE Institute of Electrical and Electronic Engineer \\ GLS Generalized Least Squares \\ LS Least Squares \\ OCFR Open Circuit Frequency Response \\ OLFR On Line Frequency Response \\ PRBS Pseudo Random Binary Sequence \\ SEP Sistemas Elétricos de Potência \\ SSFR Stand Still Frequency Response \\ SVD Singular Value Decomposition \\ WLS Weighted Least Squares \\ PSS Power System Stabilizer
}




\title{
Lista de Símbolos
}

\author{
$e_{f d} \quad$ Tensão de campo \\ $e_{d} \quad$ Tensão de armadura de eixo direto \\ $e_{q} \quad$ Tensão de armadura de eixo em quadratura \\ $E_{o} \quad$ Valor eficaz da tensão em vazio da máquina síncrona \\ $G_{s} \quad$ Função de transferência de Campo a Armadura \\ $L_{l} \quad$ Indutância de dispersão (por fase) do estator \\ $L_{a d} \quad$ Indutância de magnetização da armadura segundo \\ o eixo direto \\ $L_{a q} \quad$ Indutância de magnetização da armadura segundo \\ o eixo em quadratura \\ $L_{f d} \quad$ Indutâncias de dispersão do enrolamento de campo \\ $L_{1 q}, L_{2 q}$ Indutância de dispersão dos enrolamentos amortecedores \\ $R_{1 q}, R_{2 q}$ Resistências dos enrolamentos amortecedores segundo \\ o eixo em quadratura \\ $R_{a} \quad$ Resistência de armadura \\ $R_{f d} \quad$ Resistência do circuito de campo \\ $s=j w \quad$ Operador de laplace \\ w Freqüência elétrica em rad/s \\ $Z_{d}(s) \quad$ Impedância operacional do eixo direto \\ $Z_{q}(s) \quad$ Impedância operacional do eixo em quadratura \\ $Z_{a f o}(s) \quad$ Impedância de transferência de campo a armadura no teste SSFR
}




\section{Conteúdo}

Dedicatória

\begin{tabular}{|ll}
\hline Agradecimentos & v \\
\hline
\end{tabular}

Resumo ix

Abstract xi

Lista de Figuras xii

$\begin{array}{ll}\text { Lista de Tabelas } & \text { xvi }\end{array}$

Lista de Abreviaturas e Siglas xix

Lista de Símbolos $\quad$ xxi

\begin{tabular}{llr}
\hline 1 & Introdução & 1
\end{tabular}

1.1 Importância da Estimação de Parâmetros . . . . . . . . . . . . . . 1

1.2 Características desejáveis do processo de estimação de parâmetro 2

1.3 Objetivos do Trabalho . . . . . . . . . . . . . . . . . . . 3

$1.3 .1 \quad$ Objetivo Geral . . . . . . . . . . . . . . . . . . 3

1.3 .2 Objetivos Específicos . . . . . . . . . . . . . . . . . 3

1.4 Estrutura Metodológica do Trabalho . . . . . . . . . . . . . . . 4

1.5 Organização da dissertação . . . . . . . . . . . . . . . . . . . . 4

$\begin{array}{lll}2 & \text { Revisão Bibliográfica } & 7\end{array}$

2.1 Introdução . . . . . . . . . . . . . . . . . . . . . . . . 7 
2.2 Classificação dos testes para estimação de parâmetros em geradores elétricos . . . . . . . . . . . . . . . . 8

$2.2 .1 \quad$ Em relação a dinâmica no qual o teste é realizado . . . . . 8

$2.2 .2 \quad$ Em relação ao procedimento do teste . . . . . . . . . . . . 9

2.2.3 Em relação ao domínio no qual o teste é realizado . . . . . 9

2.2.4 Em relação a técnica na estimação de parâmetros utilizada 10

2.3 Testes em regime transitório no domínio do tempo . . . . . . . . . 11

2.3 .1 Testes de Curto Circuito . . . . . . . . . . . . . . . . . 11

2.4 Testes de Rejeição de Carga . . . . . . . . . . . . . . . . . . . . . 16

2.4 .1 Testes Usando Perturbações $\ldots . . . \quad \ldots$

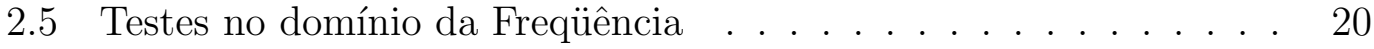

2.5 .1 Definições $\ldots \ldots \ldots \ldots$

2.5 .2 Resposta em freqüência com rotor parado (SSFR) . . . . . 22

2.5.3 Resposta em Freqüência em circuito aberto (OCFR). . . . 27

2.5 .4 Resposta de Freqüência 'on-line' (OLFR) . . . . . . . . . . 27

2.5 .5 Outras técnicas . . . . . . . . . . . . . . . . . 28

2.6 Quadro Comparativo . . . . . . . . . . . . . . . . . 28

3 Estimação de parâmetros baseada na técnica de sensibilidade de

trajetória $\quad 31$

$3.1 \quad$ Introdução . . . . . . . . . . . . . . . . . . . . . . . . 31

3.2 Funções de Sensibilidade de Trajetória $\ldots$. . . . . . . . . . . . . . 32

3.2.1 Funções de Sensibilidade de Trajetória aplicadas a um sistema linear invariante no tempo (LTI) . . . . . . . . . . 34

3.2.2 Funções de Sensibilidade de Trajetória para modelos não lineares . . . . . . . . . . . . . . . 35

3.3 Esquema de Aplicação da metodologia de sensibilidade da trajetória 36 $3.3 .1 \quad$ Processo de Minimização de Erro . . . . . . . . . . . . . . 36

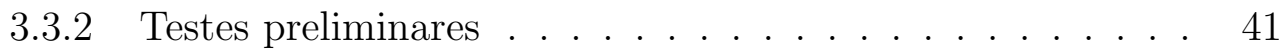


4.1 Introdução . . . . . . . . . . . . . . . . . . . . . . . . . . 49

4.1 .1 Sincronização de Sistemas $\ldots \ldots \ldots$. . . . . . . . . . 51

5 Modelagem da Máquina Síncrona e dos Controladores Associados

$5.1 \quad$ Modelagem da Máquina Síncrona . . . . . . . . . . . . . . . 57

$5.1 .1 \quad$ Equações Elétricas da Máquina Síncrona . . . . . . . . . . 59

5.1 .2 Equações Mecânicas . . . . . . . . . . . . . . . . 65

$5.1 .3 \quad$ Modelos Simplificados . . . . . . . . . . . . . . . . 68

5.1 .4 Modelo de dois eixos $\ldots \ldots \ldots$. . . . . . . . . . . . . . 69

5.1 .5 Modelo de um eixo . . . . . . . . . . . . . . . . 70

5.1 .6 Modelo Clássico . . . . . . . . . . . . . . . . . . . . . . 70

5.1 .7 Modelagem final da máquina síncrona . . . . . . . . . . 70

5.2 Modelagem do Sistema de Excitação $\ldots$. . . . . . . . . . . . . . . 73

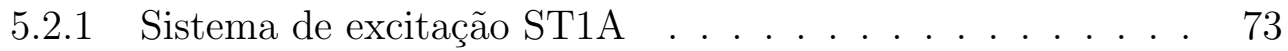

$5.2 .2 \quad$ Sistema de excitação DC1 . . . . . . . . . . . . . . . . 74

6 Aplicação da técnica de sensibilidade de trajetória para a estimação de parâmetros do gerador síncrono e do sistema de ex$\begin{array}{ll}\text { citação } & 77\end{array}$

6.1 Introdução . . . . . . . . . . . . . . . . . . . . . . . 77

6.1 .1 Considerações práticas para a realização dos testes . . . . 78

6.1 .2 Modelo do Sistema de Potência . . . . . . . . . . . . . 80

6.2 Estimação de parâmetros da máquina síncrona . . . . . . . . . . . 80

6.2 .1 Parâmetros do modelo clássico . . . . . . . . . . . . . . . . 80

6.2 .2 Estimação dos parâmetros do modelo de 'um eixo' . . . . . 86

6.3 Determinação dos parâmetros do modelo de dois eixos . . . . . . . 95

6.3.1 Redução da rede para a representação do modelo de 'dois eixos' . . . . . . . . . . . . . . . . . . 95

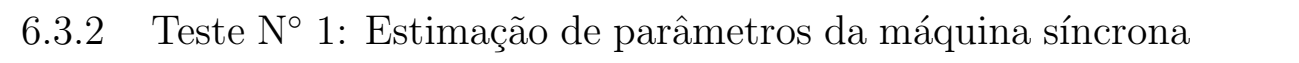
considerando como entradas $I_{d}$ e $I_{q}$ e $E_{f d}$, e como saida $w \quad 100$ 
6.3.3 Teste $\mathrm{N}^{\circ}$ 2: Estimação de parâmetros da máquina síncrona considerando $E_{f d}$ como entrada e $w$ como saída . . . . . . 109

6.3.4 Teste $\mathrm{N}^{\circ}$ 3: Estimação de parâmetros da máquina síncrona considerando $E_{f d}$ como entrada e $\delta$ e $w$ como saída . . . . 112

6.3.5 Teste $\mathrm{N}^{\circ}$ 4: Estimação considerando com o conceito de sincronização . . . . . . . . . . . . . . . . . . . . 113

6.3 .6 Estimação dos parâmetros mecânicos H e D . . . . . . . . 121

6.4 Parâmetros do Sistema de Excitação . . . . . . . . . . . . . . . . 123

6.4 .1 Estimação do sistema de excitação ST1A . . . . . . . . . . 123

6.4.2 Estimação dos parâmetros do sistema de excitação DC1. . 125

\begin{tabular}{lll}
\hline 7 & Conclusões & 131 \\
\hline
\end{tabular}

A Influência dos parâmetros da máquina síncrona e do sistema de excitação na estabilidade transitória 143

A.1 Introdução . . . . . . . . . . . . . . . . . . . . . . . . 143

A.2 Testes Realizados . . . . . . . . . . . . . . . . . . . . . 146

A.2.1 Sistema de Potência com parâmetros verdadeiros . . . . . 148

A.2.2 Influência dos parâmetros do sistema de excitação . . . . . 149

A.2.3 Influência dos parâmetros elétricos da máquina síncrona 149

A.2.4 Influência dos parâmetros mecânicos da máquina síncrona 151

\begin{tabular}{|ll|l}
\hline B Publicações Originadas desta Pesquisa & 153
\end{tabular} 


\section{Capítulo 1}

\section{Introdução}

\subsection{Importância da Estimação de Parâmetros}

Nos estudos de análise de contingência, proteção, e estabilidade de sistemas elétricos de potência (SEP) diversas simulações numéricas são realizadas para prever o comportamento em regime transitório do sistema. Com base nestas simulações, os engenheiros tomam decisões, tais como a imposição de limites de operação, ajuste dos esquemas de proteção e alívio de carga. Para que estas decisões sejam tomadas de forma a garantir a operação segura e eficiente do SEP, é necessário que as simulações descrevam satisfatoriamente o comportamento real do sistema. Para isto, os parâmetros dos modelos utilizados nestas simulações devem estar identificados corretamente. Entretanto, verificam-se incertezas em relação ao conhecimento dos parâmetros (gerador, sistema de excitação, regulador de velocidade, turbina). Estas incertezas existem por diversas razões, como: perda dos dados (dispositivos antigos), reajuste do ganho de algum componente não documentado, variação de parâmetros devido ao envelhecimento de componentes do sistema, etc. Além disso, os parâmetros fornecidos pelos fabricantes são obtidos de testes com a máquina fora de serviço ('off-line'), assim os parâmetros não levam em consideração a influência da condição de operação.

O uso de parâmetros inadequados pode fazer que o sistema opere fora da margem de segurança. Por outro lado, o uso de parâmetros mais exatos (nos modelos) pode aumentar a margem de transferência de potência trazendo benefícios 
econômicos ao sistema. A referência (Zhengming et al., 1995), por exemplo, indica que adotando novos parâmetros (após a aplicação de uma metodologia de estimação) a margem de estabilidade do gerador pode melhorar em até $5 \%$, resultando uma potência extra entre 30-50 MVA em um sistema de capacidade instalada de 675 MVA.

Outra importante aplicação do processo de estimação de parâmetros encontrase na sintonia dos controladores do gerador elétrico. Um exemplo de controlador é o estabilizador de sistema de potência, PSS (do inglês, Power System Stabilizer). O conhecimento impreciso dos parâmetros do sistema de excitação pode resultar em uma sintonia dos PSS's ineficiente ou até prejudicial à operação do SEP.

Além disso, a identificação correta dos parâmetros da máquina síncrona pode ser utilizada para a detecção de defeitos no sistema, pois a variação no valor de algum parâmetro pode ser um indicativo da existência de alguma anomalia no seu funcionamento (sendo necessário o monitoramento de alguns dos parâmetros).

\subsection{Características desejáveis do processo de es- timação de parâmetro}

Existem diversas metodologias de estimação de parâmetros propostas na literatura, cada uma com suas vantagens e desvantagens para aplicação em sistemas reais. Entretanto, poucas são adequadas para a estimação de parâmetros do gerador (e controladores associados) em operação, ou seja, sem isolar o gerador do resto do sistema ('on-line'). A parada do gerador é indesejável porque traz perdas econômicas para a empresa geradora. Partindo-se deste ponto de vista, as seguintes características são desejadas de um processo de estimação de parâmetros.

1. Realização de testes 'on-line': testes com o gerador em operação, isto é, que não requeiram o desligamento da unidade geradora do resto do sistema.

2. Seleção de variáveis para medidas de fácil acesso: os parâmetros devem 
ser determinados com as medidas disponíveis. Em geral, é necessário fazer transformações nas equações diferenciais originais para encontrar outras novas equações com variáveis mais fáceis de serem medidas.

3. Robustez em relação aos modelos: considerando que o modelo matemático é apenas uma representação aproximada do sistema real (dinâmicas desprezadas), a metodologia deve ser robusta e capaz de aproximar os parâmetros estimados aos valores que mais se aproximem aos valores verdadeiros.

4. Tratamento adequado das não linearidades: o processo de estimação deve ser capaz de representar as não linearidades presentes nos sistemas, como a saturação na máquina síncrona, os limites do sistema de excitação, entre outros.

\subsection{Objetivos do Trabalho}

\subsubsection{Objetivo Geral}

O presente trabalho tem como objetivo elaborar uma metodologia de estimação de parâmetros do gerador síncrono e seu sistema de excitação baseado na técnica de sensibilidade de trajetória e que na medida do possível atenda os requisitos desejados mencionados anteriormente.

\subsubsection{Objetivos Específicos}

- Verificar quais parâmetros podem ser estimados a partir das medidas disponíveis.

- Propor testes adequados para estimação dos parâmetros desejados.

- Estimar os parâmetros do gerador síncrono e do sistema de excitação, quando se têm incertezas nos dados.

- Estudar a viabilidade da realização de testes 'on-line' que permitam a estimação de parâmetros do gerador sem a necessidade de desconectá-lo da 
rede.

- Ter um conhecimento mais exato do comportamento transitório do sistema elétrico de potência, depois da estimação dos parâmetros.

\subsection{Estrutura Metodológica do Trabalho}

O trabalho consiste na elaboração de uma metodologia para estimação de parâmetros. Com este objetivo diferentes técnicas para estimação de parâmetros, tanto do gerador síncrono como do sistema excitação e de outros componentes (PSS e Turbinas) relatadas na literatura, foram estudadas. Dentre elas a técnica de sensibilidade da trajetória foi escolhida por permitir representar as não linearidades como, por exemplo, os limites do sistema de excitação, e a saturação da máquina síncrona assim como a possibilidade de ser utilizada em testes 'on-line'.

É importante mencionar que a metodologia de estimação de parâmetros estudada neste trabalho é adequada não apenas na área de engenharia elétrica, mas também na engenharia mecânica, hidráulica, etc. Em principio, os parâmetros de qualquer sistema que possua entradas e saídas e que seja modelado por um conjunto de equações diferenciais conhecidas podem ser estimados pela metodologia proposta.

Por fim, uma vez determinados os parâmetros, faz-se uma análise comparativa da influência da estimação dos parâmetros na estabilidade transitória. Para isto, simulações computacionais com os parâmetros originais (antes da estimação) e com os parâmetros estimados são realizadas. Esta análise servirá para determinar a influência e a importância de alguns dos parâmetros no comportamento dinâmico do sistema.

\subsection{Organização da dissertação}

Este trabalho está estruturado em sete capítulos, conforme pode ser observado na figura (1.1). 
No capítulo 2 apresenta-se uma ampla revisão bibliográfica sobre as diferentes metodologias de estimação de parâmetros relatadas na literatura. Neste capítulo, realiza-se uma classificação de algumas metodologias de estimação de parâmetros da máquina síncrona. São analisadas as vantagens e desvantagens de cada um delas.

No capítulo 3 apresenta-se a base teórica da técnica de sensibilidade de trajetória para a aplicação em sistemas lineares e não lineares. Um exemplo simples consistindo de um sistema massa-mola é utilizado para ilustrar de maneira clara o funcionamento desta técnica.

No capítulo 4 apresenta-se uma proposta para melhorar a metodologia de estimação de parâmetros. A proposta está baseada na teoria de sincronização de sistemas. Neste capítulo é dada a base teórica e as vantagens de seu uso no processo de estimação.

No capítulo 5 são feitas algumas considerações sobre máquinas síncronas, circuitos equivalentes e modelos usualmente utilizados na análise de estabilidade transitória. São detalhadas as equações diferenciais da máquina síncrona (modelo clássico, modelo de 1 eixo, e modelo de 2 eixos) e do sistema de excitação (tipo ST1A e DC1, segundo (IEEE, 1992)).

No capítulo 6 são apresentados os testes realizados para estimar os parâmetros da máquina síncrona e do sistema de excitação utilizando a técnica de sensibilidade de trajetória. No caso da máquina síncrona, relatam-se alguns testes realizados para estimação simultânea de vários parâmetros. Também apresenta-se a aplicação da técnica de sincronização de sistemas no processo de estimação de parâmetros.

No capítulo 7, são apresentados as conclusões da dissertação.

Por fim, no apêndice 1, analisa-se a influência dos parâmetros da máquina síncrona e seu sistema de excitação na estabilidade transitória. Para tal objetivo são realizados testes de estabilidade em um pequeno sistema de potência sujeito a incertezas nos parâmetros. No apêndice 2 são apresentados os programas computacionais desenvolvidos no presente trabalho. 


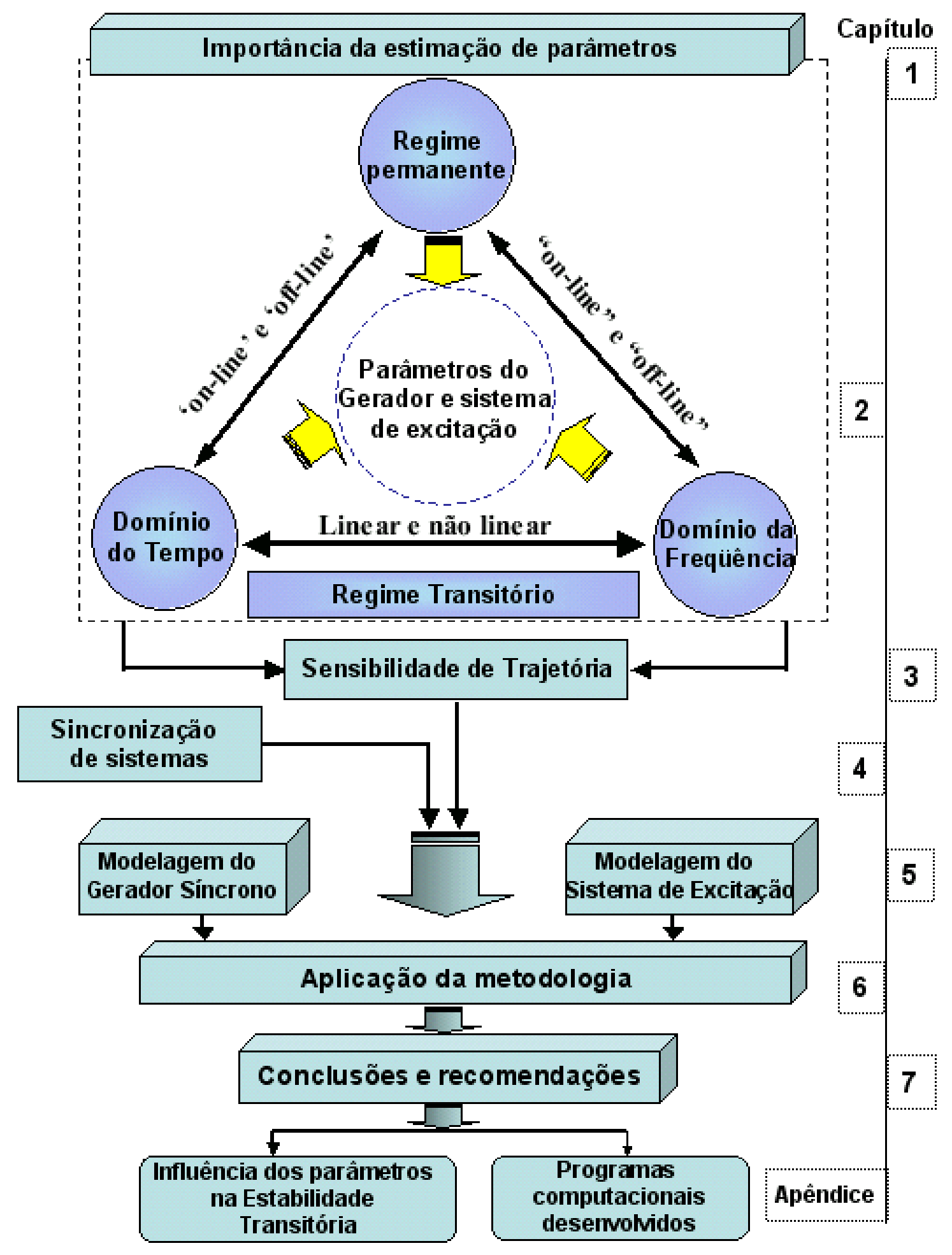

Figura 1.1: Estrutura Metodológica da dissertação 


\section{Capítulo 2}

\section{Revisão Bibliográfica}

\subsection{Introdução}

Neste capítulo apresentam-se as principais características de diferentes metodologias de estimação de parâmetros encontradas na literatura, algumas das quais foram padronizadas pelo IEEE (do inglês, Institute of Electrical and Electronic Engineer) em (IEEE, 1995) e (IEEE, 2002).

Embora as metodologias relatadas sejam para a estimação de parâmetros da máquina síncrona, por intermédio de testes e técnicas similares, muitos autores propuseram metodologias similares para a estimação de parâmetros do sistema de excitação e de turbinas.

Na próxima seção, realiza-se uma classificação de diversas metodologias de estimação de parâmetros segundo vários critérios. Pode-se mencionar que as metodologias de estimação de parâmetros constam basicamente de duas etapas. Na primeira etapa, realiza-se o teste para obter a resposta do gerador ante uma entrada. Isto é realizado no laboratório ou no campo (por exemplo, na usina elétrica). Na segunda etapa, os parâmetros são estimado com os dados obtidos na primeira etapa. Neste caso, utiliza-se geralmente de alguma técnica de minimização de erro. 


\subsection{Classificação dos testes para estimação de parâmetros em geradores elétricos}

As metodologias para estimação de parâmetros de máquinas síncrona podem ser classificadas segundo vários critérios, aqui consideram-se:

1. Em relação ao regime de operação no qual o teste é realizado: permanente ou transitório.

2. Em relação ao procedimento do teste: 'On-line' ou 'Off-line'.

3. Em relação ao domínio no qual o teste é realizado: domínio do tempo ou no domínio da freqüência.

4. Em relação à técnica utilizada na estimação de parâmetros: linear ou não linear.

\subsubsection{Em relação a dinâmica no qual o teste é realizado}

As metodologias podem ser classificadas em metodologias com testes em regime permanente ou em regime transitório.

\section{Testes no regime permanente}

Os testes em regime permanente são úteis para estabelecer a ordem de grandeza dos parâmetros e estimar parâmetros associados com a operação em regime permanente. Estes testes também são necessários para analisar o desempenho do gerador sob condições de regime permanente. Podem ser divididos em duas categorias: medições da curva de saturação em circuito aberto e medições da curva 'V'. A curva de saturação em circuito aberto fornece as tensões induzidas em função da excitação da máquina operando em vazio e mantida sob rotação constante. As medições da curva 'V' são realizadas com a unidade ligada à rede elétrica com uma determinada carga. Nessas condições, varia-se a excitação da máquina 
para variar a potência reativa. Através destes testes os seguintes parâmetros do gerador podem ser estimados:

- $X_{d}$ Reatância síncrona de eixo direto.

- $X_{a d}$ Reatância mútua de eixo direto, a qual é uma fração de $X_{d} . X_{d}=$ $X_{a d}+X_{l}$, onde $X_{l}$ é a reatância de dispersão (por fase) da armadura.

- $X_{q}$ Reatância síncrona de eixo em quadratura.

- $X_{a q}$ Reatância mútua de eixo em quadratura, a qual é uma fração de $X_{q}$. $X_{q}=X_{a q}+X_{l}$

\section{Testes em regime transitório}

Os testes em regime transitório consistem em provocar uma perturbação (no sistema) simples e segura para obter a resposta transitória da máquina. A partir desta resposta obtém-se os ganhos e constantes de tempo da máquina síncrona muitas dos quais não podem ser obtidos a partir dos testes em regime permanente.

\subsubsection{Em relação ao procedimento do teste}

As metodologias são baseadas em testes 'on-line' e 'off-line'. Os testes 'on-line' são realizados com o gerador em operação (gerador ligado à rede). Isto é uma característica desejável pois a parada do gerador está associado a perdas econômicas. Além disso os parâmetros estimados levam em consideração o ponto de operação da máquina. Por sua vez, os testes 'off-line' são realizados com o gerador fora de operação (gerador desligado da rede).

\subsubsection{Em relação ao domínio no qual o teste é realizado}

As metodologias podem ser classificados em metodologias com testes no domínio do tempo e no domínio da freqüência. Esta classificação é considerada a mais abrangente. 


\subsubsection{Em relação a técnica na estimação de parâmetros utilizada}

As metodologias podem ser classificadas em metodologias com técnicas lineares

e técnicas não lineares.

As técnicas lineares transformam as equações originais em um conjunto de equações lineares. As técnicas não lineares utilizam métodos não lineares para realizar o ajuste dos parâmetros.

Dentre as técnicas tem-se:

1. Estimador de Mínimos Quadrados (técnica linear) utilizadas em (Le e Wilson, 1998) e as variações, mínimos quadrados ponderados utilizadas em (Liu et al., 1993) e mínimos quadrados generalizados utilizadas em (Guo et al., 1995)).

2. Ajuste de Curvas (técnica não linear) como o ajuste polinomial proposto em (IEEE, 1995).

3. Sensibilidade da Trajetória (técnica não linear) (Benchluch e Chow, 1993), (Sanchez et al., 1988) e (Hiskens e Koeman, 1998).

4. Utilização de séries (técnica não linear) como as séries Hartley (Melgoza et al., 2001).

5. técnica baseada em gradiente apresentada em (Zali et al., 2000).

A técnica mais utilizada é o estimador de mínimos quadrados LSE (do inglês, Least Squares Estimator). Em (Le e Wilson, 1998), o estimador mínimos quadrados foi utilizada para a estimação de parâmetros da máquina síncrona. O estimador de mínimos quadrados clássico, penaliza igualmente os erros cometidos para cada medida. Em algumas situações práticas deseja-se dar pesos diferentes para certos valores de erros. Pode ocorrer que por um determinado período de tempo o grau de incerteza das medições seja maior (presença de ruído). Neste caso, pode-se dar um peso maior aos erros cometidos nesse período de tempo. 
Nessa situação, utiliza-se o estimados de mínimos quadrados ponderados WLS (do inglês, Weight Least Squares). Em (Liu et al., 1993) o estimador de mínimos quadrados ponderados foi utilizado para determinar os parâmetros do sistema de excitação. Uma outra variação da técnica LSE é o estimador de mínimos quadrados generalizados GLS (do inglês, Generalization Least Squares), que fornece uma melhor precisão na presença de ruídos correlacionados (Guo et al., 1995). Esta técnica foi utilizada na determinação de parâmetros do sistema de excitação e do PSS em (Guo et al., 1995).

\subsection{Testes em regime transitório no domínio do tempo}

\subsubsection{Testes de Curto Circuito}

Os primeiros testes no domínio do tempo relatados na literatura foram os testes de curto circuito. Antes da aplicação do curto circuito, o gerador síncrono deve estar operando sem carga e à velocidade síncrona. Como a máquina está sem carga, apenas existirão correntes fluindo no enrolamento de campo.

Após aplicado o curto circuito, aparecem correntes no enrolamento de armadura que criam, por sua vez, uma força magnetomotriz $\left(F_{a}\right)$ opondo-se à força magnetomotriz original $\left(F_{f}\right)$. O surgimento das correntes de curto circuito no estator e o efeito desmagnetizante de $F_{a}$ são acompanhados de um incremento na corrente de campo, governado pela lei de Lenz, que tenta manter o fluxo concatenado em seus valores iniciais. Estas correntes criam um nova componente da força magnetomotriz que se adiciona à $F_{f}$ tendendo neutralizar a desmagnetização resultante de $F_{a}$. Se esta situação persistir indefinidamente, a máquina passará a uma nova condição de regime permanente, agora de curto circuito. Na figura 2.1 mostra-se o decaimento da corrente da armadura na fase 'a' e no enrolamento de campo depois de aplicado o curto circuito nos terminais da máquina.

Cada corrente de armadura consiste em componentes simétricas (alternadas) e assimétricas (componentes contínuas). A componente AC das três correntes de 


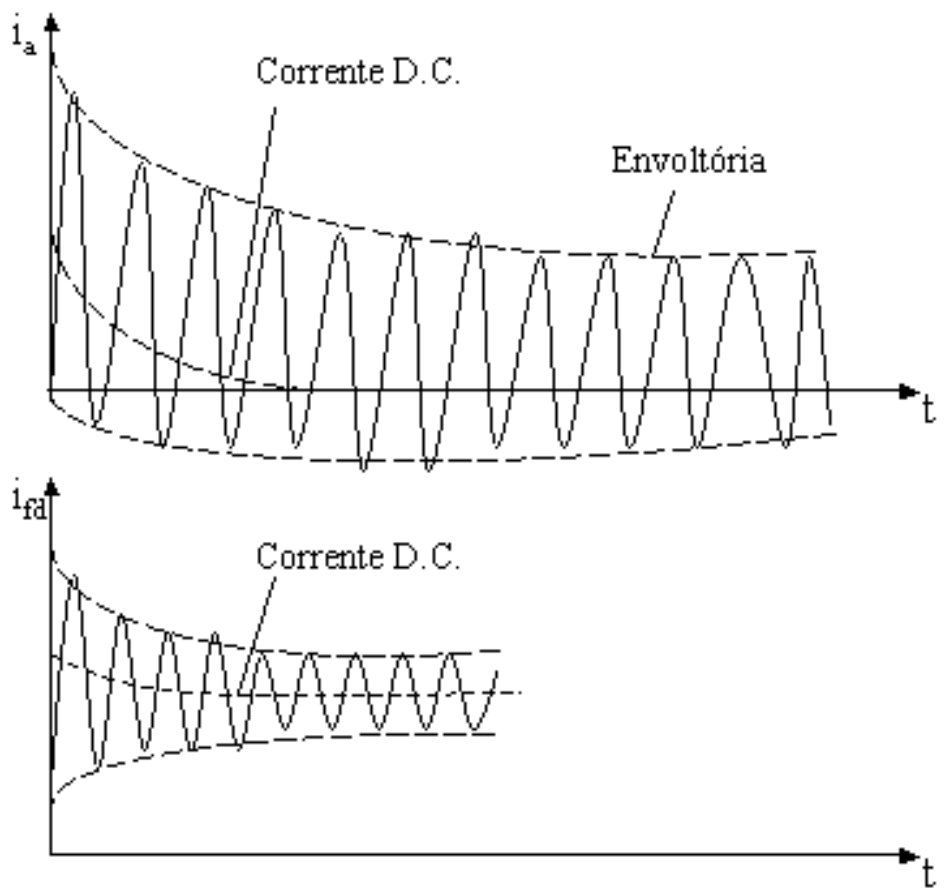

Figura 2.1: Corrente de curto circuito na fase a $I_{a}$, e no enrolamento de campo $I_{f d}$

armadura tem envoltórios idênticos como mostra a figura (2.2).

Estes envoltórios decaem de um valor inicial no instante do curto circuito a um valor final permanente $I_{s}$. Os valores $\mathrm{AC}$ (pico) e DC são obtidos como a metade da diferença e a metade da soma dos envoltórios superior e inferior. Os pontos correspondentes ao valor eficaz são obtidos dividindo-se o valor de pico por $\sqrt{2}$. Subtraindo $I_{s}$ da forma de onda AC, o restante consiste em duas componentes exponenciais: a componente transitória I', com uma constante de tempo $T_{d}^{\prime}$, e uma componente sub-transitória I", com uma constante de tempo muito pequena $T_{d}^{\prime \prime}$. Assim a corrente $\mathrm{AC}$ é dada pela seguinte equação:

$$
I_{a c}=I_{s}+I_{o}^{\prime} e^{-\frac{t}{T_{d}^{\prime}}}+I_{o}^{\prime \prime} e^{-\frac{t}{T_{d}^{\prime \prime}}}
$$

sendo:

- $I_{s}$ : corrente eficaz de curto-circuito em regime permanente.

- $I_{o}^{\prime}$ : o valor eficaz inicial da componente alternativa transitória da corrente 


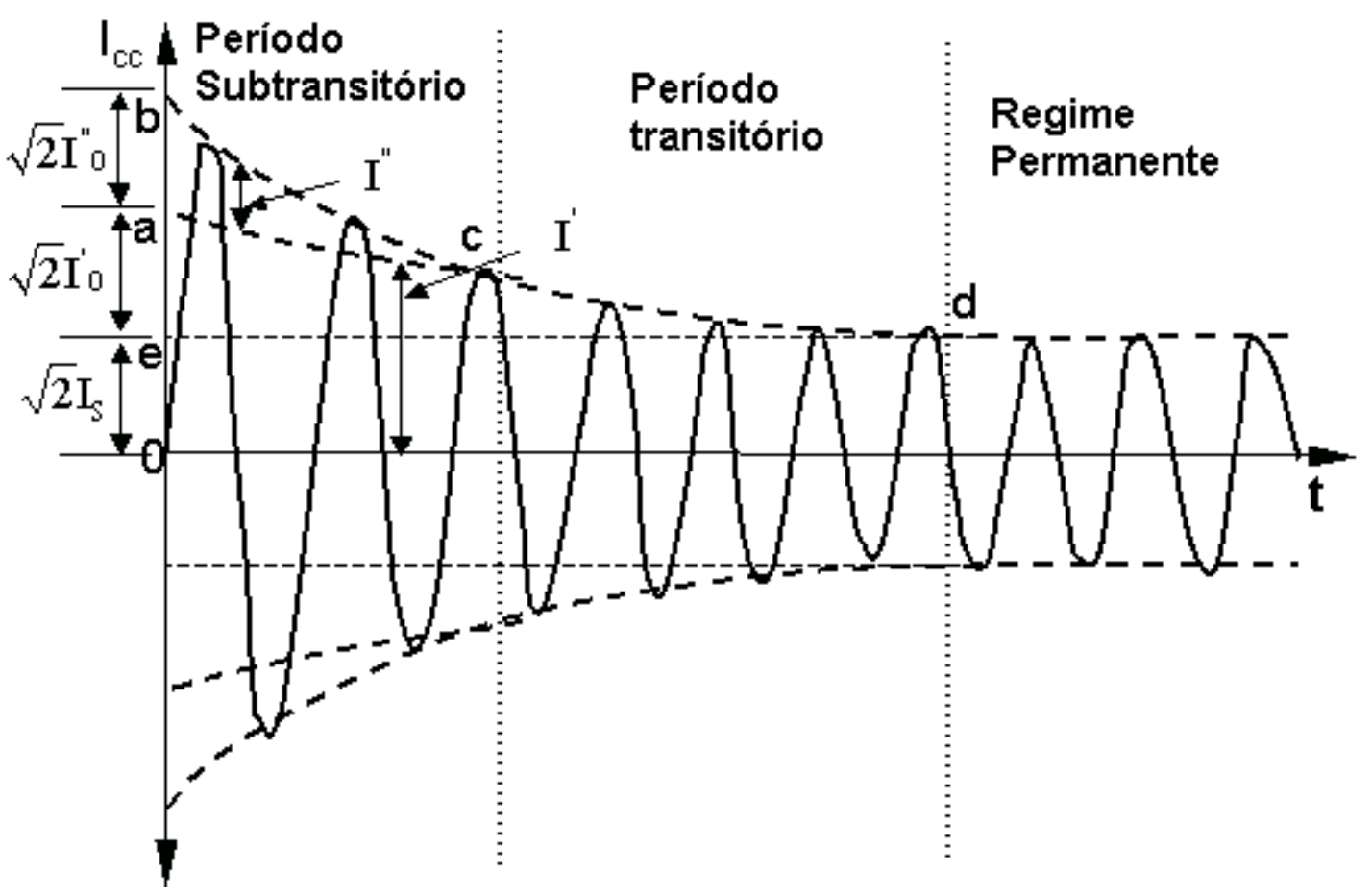

Figura 2.2: Resposta da corrente da fase 'a' após um curto circuito no estator.

de curto circuito.

- $I_{o}^{\prime \prime}$ : o valor eficaz inicial da componente alternativa subtransitória da corrente de curto circuito.

As amplitudes das componentes AC da corrente de armadura podem ser calculadas em termos das reatâncias de eixo direto $x_{d}, x_{d}^{\prime}$ e $x_{d}^{\prime \prime}$ pelo princípio de superposição. Assim tem-se

$$
\begin{aligned}
& x_{d}=\frac{E_{o}}{I_{s}} \\
& x_{d}^{\prime}=\frac{E_{o}}{I_{s}+I_{o}^{\prime}} \\
& x_{d}^{\prime \prime}=\frac{E_{o}}{I_{s}+I_{o}^{\prime}+I_{o}^{\prime \prime}} \\
& I_{a c}=E_{o}\left(\frac{1}{x_{d}}+\left(\frac{1}{x_{d}^{\prime}}-\frac{1}{x_{d}}\right) e^{-\frac{t}{T_{d}^{\prime}}}+\left(\frac{1}{x_{d}^{\prime \prime}}-\frac{1}{x_{d}^{\prime}}\right) e^{-\frac{t}{T_{d}^{\prime \prime}}}\right)
\end{aligned}
$$

sendo $E_{o}$ o valor eficaz da tensão em vazio da máquina síncrona antes do curto circuito. 
A componente DC das três correntes de armadura são, geralmente, de magnitudes diferentes, elas decrescem exponencialmente a zero com a mesma constante de tempo $T_{a}$. Estes são dadas por:

$$
I_{D C}=\frac{\sqrt{2} E_{f} \cos (\alpha)}{X_{d}^{\prime \prime}} e^{\frac{-t}{T_{a}}}
$$

sendo $\alpha$ o ângulo de corte no período AC no instante que ocorre o curto circuito (ângulo de incidência).

A componente transitória e a componente subtransitória podem ser desenhadas em uma escala semi-logarítmica. Desse modo, os envoltórios ac e bcd da figura (2.2) aproximam-se de retas como mostra a figura (2.3).

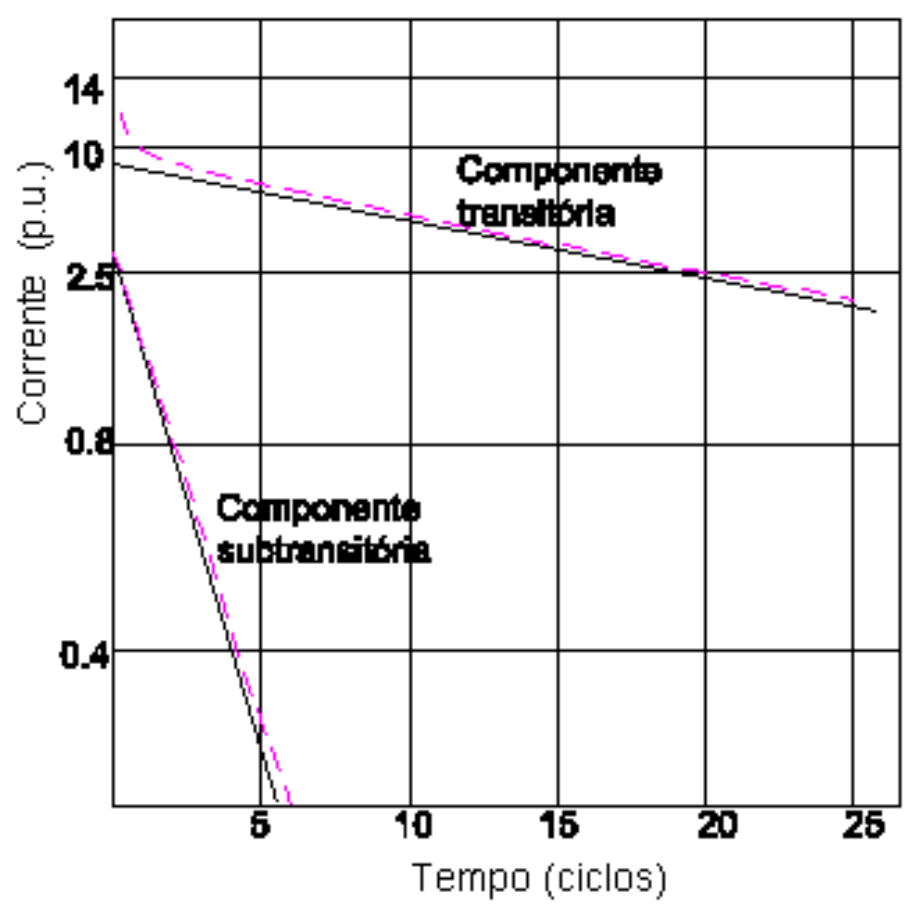

Figura 2.3: Componentes da corrente de curto circuito em escala semi-logarítmica

Dos diagramas logarítmicos da figura 2.4, as constantes de tempo em eixo direto $T_{d}^{\prime}, T_{d}^{\prime \prime}$, e a constante de tempo de armadura $T_{a}$ podem ser obtidas graficamente como segue:

- $T_{d}^{\prime}$ corresponde a duas vezes o tempo necessário para que I' decresça a $1 / e^{0.5}$ (ou 0.606) vezes seu valor inicial. 
- $T_{d}^{\prime \prime}$ corresponde ao tempo necessário para que I" decresça a 1/e (ou 0.368) vezes seu valor inicial.

- $T_{a}$ corresponde ao tempo necessário para que $I_{f d}$ decresça a $1 / e$ (ou 0.368) vezes seu valor inicial. $\mathrm{O}$ valor de $T_{a}$ pode ser calculado considerando as correntes de armadura DC e correspondem ao tempo necessário para que estas correntes decresçam $1 / e$ (ou 0.368 ) vezes seus valores iniciais.

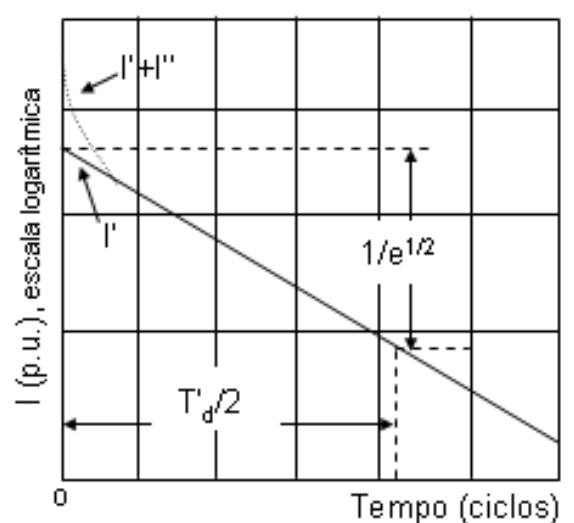

(a) Determinaçầo de $T^{\prime} d$

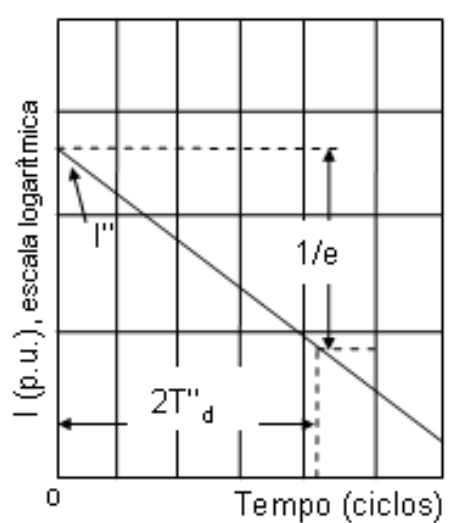

(b) Determinaçẫo de T"d

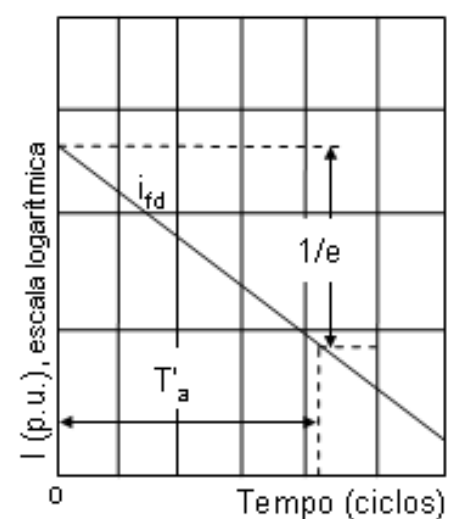

(c) Determinação de $T_{a}$

Figura 2.4: Determinação das constantes de tempo da máquina síncrona

O grande inconveniente dos testes em curto circuito é que a máquina síncrona é submetida a intenso esforço mecânico devido às correntes elevadas do teste. Além disso o método não dá informação sobre os parâmetros do eixo em quadratura e a unidade geradora tem que estar fora de operação para a realização do teste. Outro inconveniente do processo de estimação de parâmetros por testes de curto circuito é que os parâmetros são obtidos por uma análise gráfica, a qual tem um grau de imprecisão elevado. A obtenção dos envoltórios superior e inferior constitui uma aproximação que poderá acarretar grandes erros principalmente quando se utilizam modelos de ordens superiores, já que nestes casos, as constantes de tempo são muito pequenas. Um estudo visando melhorar o inconveniente da análise gráfica é apresentado em (Soliman e Alkandari, 1996). As fases deste procedimento são as seguintes: 
1. Primeiro, realiza-se o teste de curto circuito e registram-se os valores da corrente de curto circuito.

2. Em seguida, divide-se a equação da corrente de curto circuito em três componentes: estacionária, transitória, e subtransitória.

3. Estas equações são linearizadas reduzindo o problema a um conjunto de equações lineares.

4. Com os dados registrados e utilizando a técnica de otimização de mínimos quadrados determinam-se as constantes da linearização.

5. Finalmente, os parâmetros da máquina síncrona são determinados por intermédio das constantes obtidas no passo anterior.

Com esta aproximação a precisão dos parâmetros estimados é melhorada. Mesmo assim, a técnica ainda apresenta os inconvenientes do teste de curto circuito tradicional.

\subsection{Testes de Rejeição de Carga}

Outro método para identificação de parâmetros é utilizar dados de testes de rejeição de carga. Através de rejeições de carga em pontos específicos de operação, os parâmetros do eixo direto e do eixo em quadratura são obtidos (Feltes et al., 2002), (Hannett e Feltes, 1993), (Zali et al., 2000), (Bortoni e Jardini, 2002).

O teste de rejeição de carga em eixo direto requer que o gerador síncrono esteja conectado à rede com potência ativa zero e fornecendo potência reativa. Assim, existirá fluxo apenas no eixo direto. Nesta condição a corrente de armadura do gerador estará alinhada com o eixo direto $\left(i_{q}=0\right)$, como mostra a figura 2.5(a).

A máquina deve estar sub-excitada, de modo que a saturação não tenha influência. Sob essas condições a máquina é desligada do sistema. A tensão terminal e a corrente de campo decaem seguindo um comportamento similar ao teste em curto circuito e os parâmetros $x_{d}, x_{d}^{\prime}, x_{d}^{\prime \prime}, T_{d o}^{\prime}, T_{d o}^{\prime \prime}$ são obtidos. 


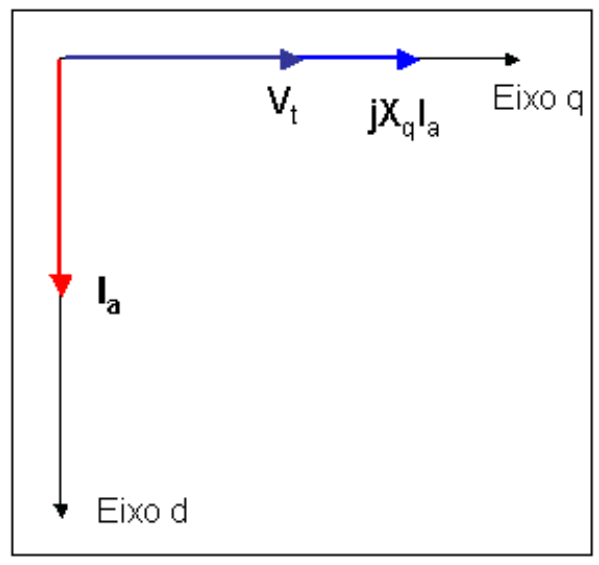

(a) Teste no eixo direto

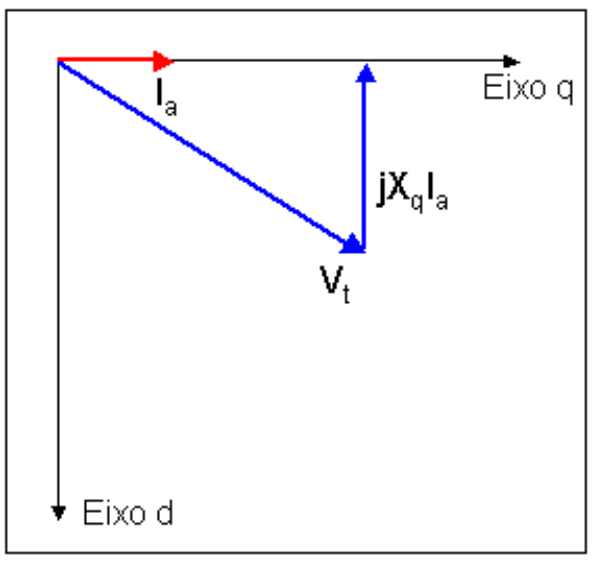

(b) Teste no eixo em quadratura

Figura 2.5: Diagrama fasorial no teste de rejeição de carga.

Na figura 2.6 mostra-se o decaimento da tensão terminal depois da rejeição de carga no eixo direto. Os parâmetros do eixo direto são calculados pelas seguintes

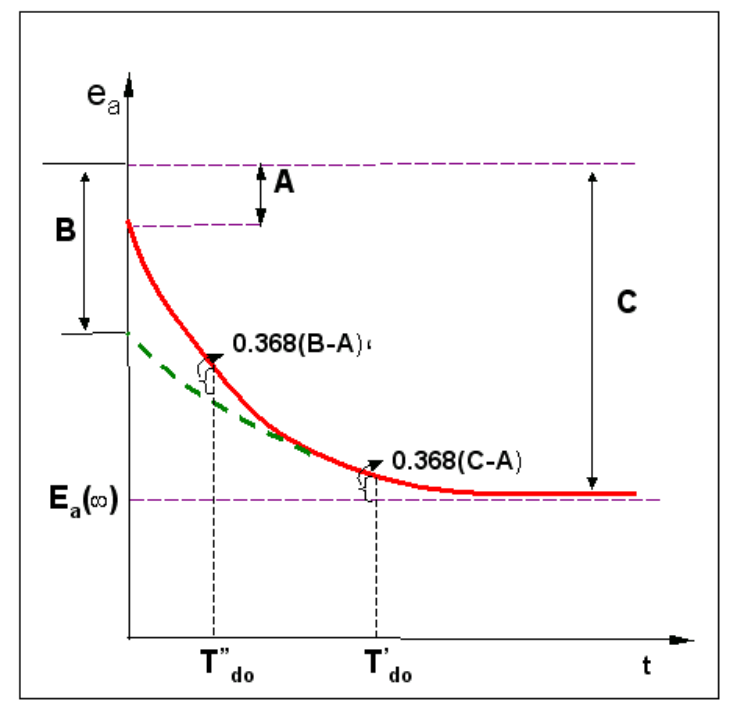

Figura 2.6: Tensão terminal da máquina síncrona após a rejeição de carga no eixo direto.

relações (Vas, 1993)

$$
x_{d}=\frac{C}{I_{d o}} \quad x_{d}^{\prime}=\frac{B}{I_{d o}} \quad x_{d}^{\prime \prime}=\frac{A}{I_{d o}}
$$

onde $I_{d o}$ é a corrente no eixo direto no instante da aplicação da perturbação. Os valores de A, B, C, podem ser obtidos da figura 2.6 . 
Para estimar os parâmetros do eixo em quadratura, a condição de operação deve ser tal que a corrente de armadura do gerador esteja completamente alinhada com o eixo em quadratura $\left(i_{d}=0\right)$ (figura $\left.2.5(\mathrm{~b})\right)$. A potência reativa é ajustada de tal maneira que o ângulo de fase entre a corrente e a tensão terminal (ângulo de fator de potência) seja igual ao ângulo de carga. Nestas condições o disjuntor é acionado e a tensão terminal e a corrente de campo são monitoradas. A figura 2.7 mostra o decaimento da tensão terminal depois da rejeição de carga no eixo em quadratura.

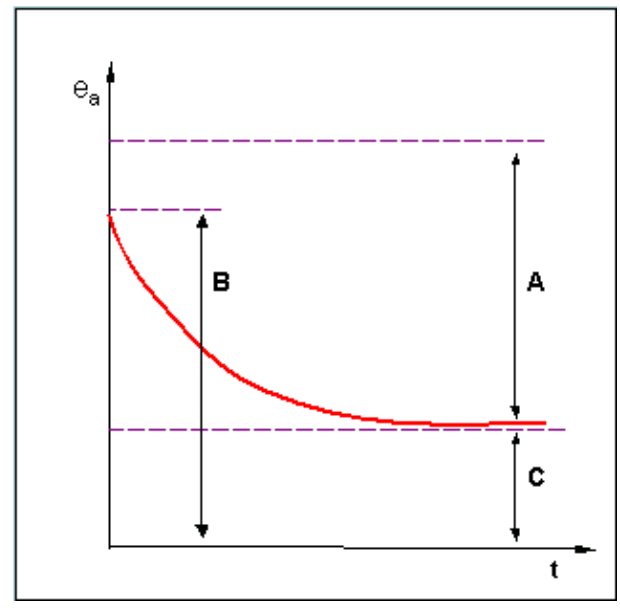

Figura 2.7: Tensão terminal da máquina síncrona após a rejeição de carga no eixo em quadratura.

Considerando as equações da máquina (Vas, 1993), as expressões de $x_{q}$ e $x_{q}^{\prime \prime}$ podem ser obtidas usando os valores medidos de A B e C da figura (2.7).

$$
x_{q}=\frac{\sqrt{A^{2}-C^{2}}}{I_{s q o}} \quad x_{q}^{\prime \prime}=\frac{\left(\sqrt{A^{2}-C^{2}}-\sqrt{B^{2}-C^{2}}\right)}{I_{s q o}}
$$

Ajustar a carga para uma condição de rejeição de carga em eixo de quadratura não é facil. Na prática, isto é conseguido por meio de sucessivas rejeições de carga com diferentes níveis de potência tentando minimizar as variações da corrente de campo. Um procedimento alternativo seria utilizar um medidor de ângulo de potência. Estas dificuldades dificultam a aplicação do teste. Considerando isto, (Bortoni e Jardini, 2002) propõe um novo teste de rejeição mas em um eixo 
arbitrário. Fazendo isto os parâmetros do eixo direto e eixo em quadratura podem ser identificados. A medição do ângulo de carga também não foi necessária pois a condição inicial da equação diferencial do ângulo da máquina foi considerado como parâmetro para ser estimado pela metodologia ali proposta.

O teste de rejeição de carga tem a vantagem de estimar parâmetros tanto do eixo direto quanto do eixo em quadratura. Entretanto, para a maioria das máquinas, é difícil alcançar condições não saturadas, o que complica o teste e a análise dos resultados. Os testes precisam de re-programação da geração e vários chaveamentos, o que não é muito prático.

\subsubsection{Testes Usando Perturbações}

Este teste consiste em aplicar perturbações na máquina síncrona para obter a sua resposta transitória. As perturbações podem ser aplicadas nos controladores como a inclusão de um degrau de tensão no sistema de excitação, ou diretamente no enrolamento de campo da máquina . Em (Vermeulen et al., 2002), (Touhami et al., 1994) e (Karrari e P., 2004), um tipo de perturbação em especial conhecida como PRBS (Pseudo Random Binary Sequence) foi utilizada. Os sinais PRBS consistem em estados binários de seqüência aleatória os quais são gerados através de um registrador realimentado. Em (Vermeulen et al., 2002), as perturbações foram aplicadas à entrada de referência do regulador automático de tensão (AVR) como mostra a figura 2.8, e em (Touhami et al., 1994) tais perturbações são aplicadas diretamente ao enrolamento de campo.

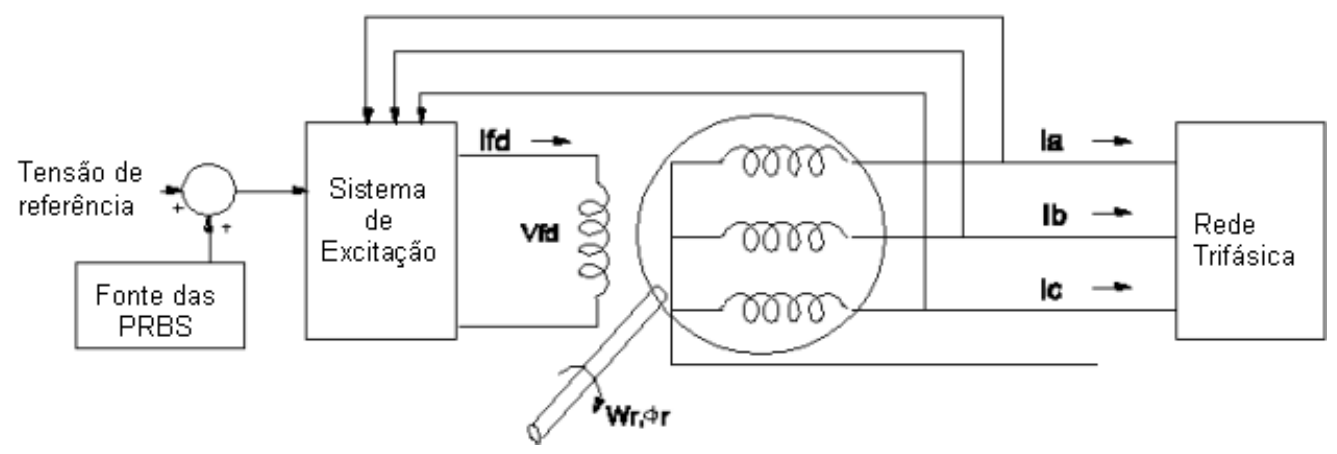

Figura 2.8: Métodologia com tese de inclução de perturbações PRBS no AVR. 
Cabe destacar que a metodologia de inclusão de perturbações aplicadas no AVR relatado em (Vermeulen et al., 2002) não permite a estimação de todos os parâmetros da máquina síncrona. Em geral, dependendo do tipo de perturbação, podem ser identificados alguns parâmetros específicos da máquina síncrona. É importante ressaltar que a perturbação não deve ser muito grande para que o gerador não perca o sincronismo, nem muito pequena para que não se tenha informação suficiente para a identificação dos parâmetros.

\subsection{Testes no domínio da Freqüência}

Os testes no domínio da freqüência descrevem a resposta em freqüência dos fluxos da máquina síncrona pelas variações da tensão do campo e as correntes do estator em ambos os eixos, direto e em quadratura. Algumas vantagens dos testes no domínio da freqüência são as seguintes :

1. Podem ser realizados no mesmo lugar onde estiver a máquina.

2. Possui baixa probabilidade de danos para a máquina testada.

3. Os parâmetros, tanto no eixo em quadratura como no eixo direto, podem ser identificados.

\subsubsection{Definições}

A seguir apresentam-se algumas definições que são importantes para a análise em resposta de freqüência. Estas definições incluem análise da transformada de Laplace na máquina síncrona e estão detalhadas em (IEEE, 2002).

- $L_{d}(s)$ Indutância operacional no eixo direto: é a transformada de Laplace da razão entre o fluxo concatenado no eixo direto da armadura e a corrente no eixo direto, com o campo em curto circuito.

- $L_{q}(s)$ Indutância operacional no eixo em quadratura: é a transformada de Laplace da razão entre o fluxo concatenado no eixo em quadratura da armadura e a corrente no eixo direto. 
- $G(s)$ Função de transferência ente o campo e a armadura: é a transformada de Laplace da razão entre o fluxo concatenado do eixo direto da armadura e tensão de campo, com a armadura em circuito aberto.

Um método conveniente para estimar características elétricas da máquina síncrona consiste em utilizar parâmetros operacionais que relacionam as grandezas da armadura e do circuito de campo. Referindo-se a figura 2.9, a relação entre variação entre as grandezas do terminal pode ser expressa pela seguinte forma operacional:

$$
\begin{aligned}
& \Delta \Psi_{d}(s)=G(s) \Delta e_{f d}(s)-L_{d}(s) \Delta i_{d}(s) \\
& \Delta \Psi_{q}(s)=-L_{q} \Delta i_{q}(s)
\end{aligned}
$$

Onde

- $\Psi_{d}$ e $\Psi_{q}$ são os fluxos concatenados do estator segundo o eixo direto e quadratura.

- $i_{d}$ e $i_{q}$ são as correntes no ponto de operação.

- $e_{f d}$ é a magnitude de tensão de campo no ponto de operação.

- $\Delta$ é uma pequena perturbação por volta do ponto de operação.

- $s$ : Operador de Laplace, $s=j w$ e $w=2 \pi f \mathrm{rad} / \mathrm{s}$.

As três principais relações mostrados a seguir, fornecem os três parâmetros definidos anteriormente.

- $Z_{d}(s)$ : Impedância operacional segundo o eixo direto, $Z_{d}=R_{a}+s L_{d}(s)$. Onde, $R_{a}$ é a resistência de armadura por fase (a contribuição de $R_{a}$ é significante apenas para baixas freqüências.). $Z_{d}(s)$ e é obtido por:

$$
Z_{d}(s)=-\left.\frac{\Delta e_{d}(s)}{\Delta i_{d}(s)}\right|_{\Delta e_{f d}=0}
$$

- $Z_{q}(s)$ : Impedância operacional segundo o eixo em quadratura. $Z_{q}=R_{a}+s L_{q}(s)$. $Z_{q}(s)$ é obtido por:

$$
Z_{q}(s)=-\frac{\Delta e_{q}(s)}{\Delta i_{q}(s)}
$$




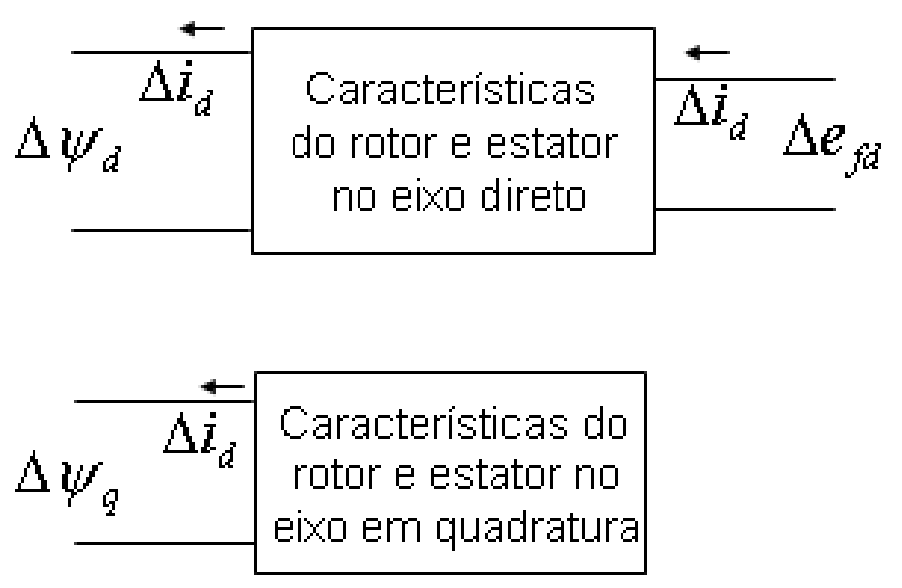

Representaçẫo no eixo direto e quadratura

Figura 2.9: Representação dos terminais no eixo direto e no eixo em quadratura baseado na equação refequa:freqfluxdq.

- A terceira relação é dada pela relação:

$$
G_{s}=-\left.\frac{\Delta e_{d}(s)}{s \Delta e_{f d}(s)}\right|_{\Delta i_{d}=0}
$$

Um quarto parâmetro que pode ser obtido em SSFR, é a impedância de transferência de campo e é dado por:

$$
Z_{a f o}=-\left.\frac{\Delta e_{f d}(s)}{\Delta i_{d}(s)}\right|_{\Delta i_{f d}=0}
$$

\subsubsection{Resposta em freqüência com rotor parado (SSFR)}

Os testes em SSFR (do inglês, Stand Still Frequency Response), são realizados com a unidade em repouso (rotor parado e isolado do sistema). O IEEE (IEEE, 1995) descreve o procedimento para a determinação de parâmetros dos testes em SSFR. O rotor deve ser alinhado em duas posições particulares com relação ao estator durante o teste.

Posicionamento do rotor para o teste de eixo direto: Conectar o amplificador de potência como mostra a figura 2.10. Acionar o amplificador a 100 $\mathrm{Hz}$ (aproximadamente), e medir a tensão de campo induzida com o osciloscópio. Girar o rotor do gerador lentamente até que a tensão de campo induzida observada no osciloscópio seja nula. Neste ponto o eixo magnético do enrolamento do 
campo estará alinhado com o eixo magnético da conexão série entre a fase 'a' e 'b', que será utilizada para o teste em eixo direto.

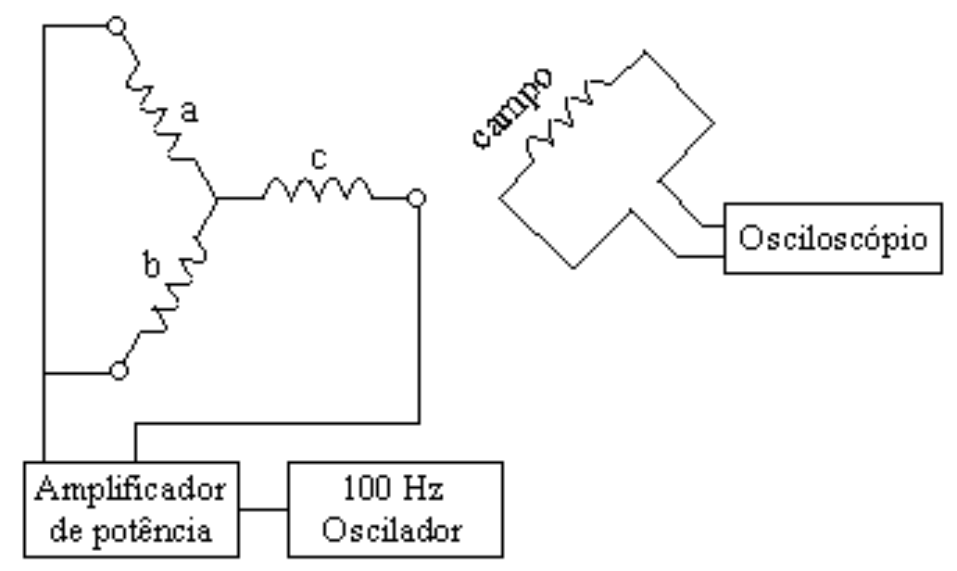

Figura 2.10: Posição do rotor para o teste em eixo direto.

Cálculo de $L_{d}(s)$ :

Conectar a saída do amplificador de potência aos terminais "a"e "b" do enrolamento de armadura através do medidor shunt como mostra a figura 2.11. Fazer um curto circuito ao enrolamento do campo através de um shunt. Conectar os sinais $v_{\text {arm }}$ e $i_{\text {arm }}$ aos instrumentos.
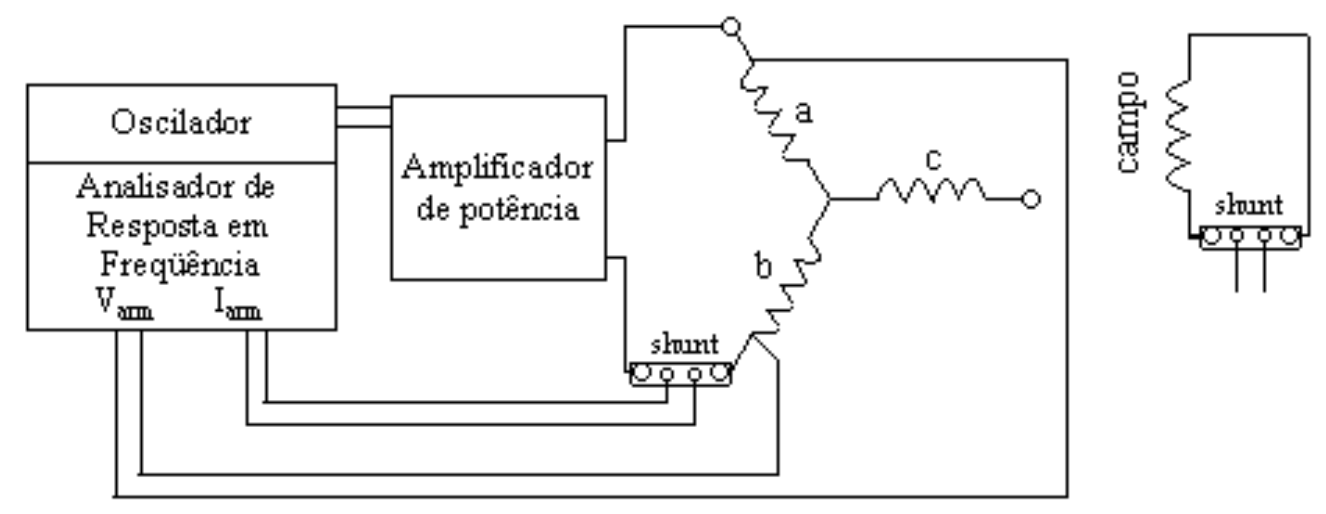

Figura 2.11: Teste para a medida de $Z_{d}$.

A seguinte relação pode ser obtida:

$$
Z_{\text {armd }}=\frac{\Delta v_{\text {arm }}(s)}{\Delta i_{\text {arm }}(s)}
$$

A indutância operacional em eixo direto $L_{d}(s)$ pode ser calculada por:

$$
L_{d}(s)=\frac{Z_{d}(s)-R_{a}}{s}
$$


Onde:

- $Z_{d}(s)=\frac{1}{2} Z_{\text {armd }}(s)$ (impedância operacional no eixo direto).

- $R_{a}=\frac{1}{2}\left\{\lim _{s \rightarrow 0}\left[Z_{\text {armd }}(s)\right]\right\}$ (Resistência de armadura).

A impedância operacional no eixo direto obtida, $Z_{d}(s)$, resulta em um conjunto de pontos similares àqueles da figura 2.12. Para obter a resistência de armadura, $R_{a}$, faz-se o gráfico da impedância $Z_{d}(s)$ (apenas a parte real) em função da freqüência e extrapola-se estes valores à freqüência zero. O valor assim obtido corresponderá à resistência entre duas fases $\left(2 R_{a}\right)$.

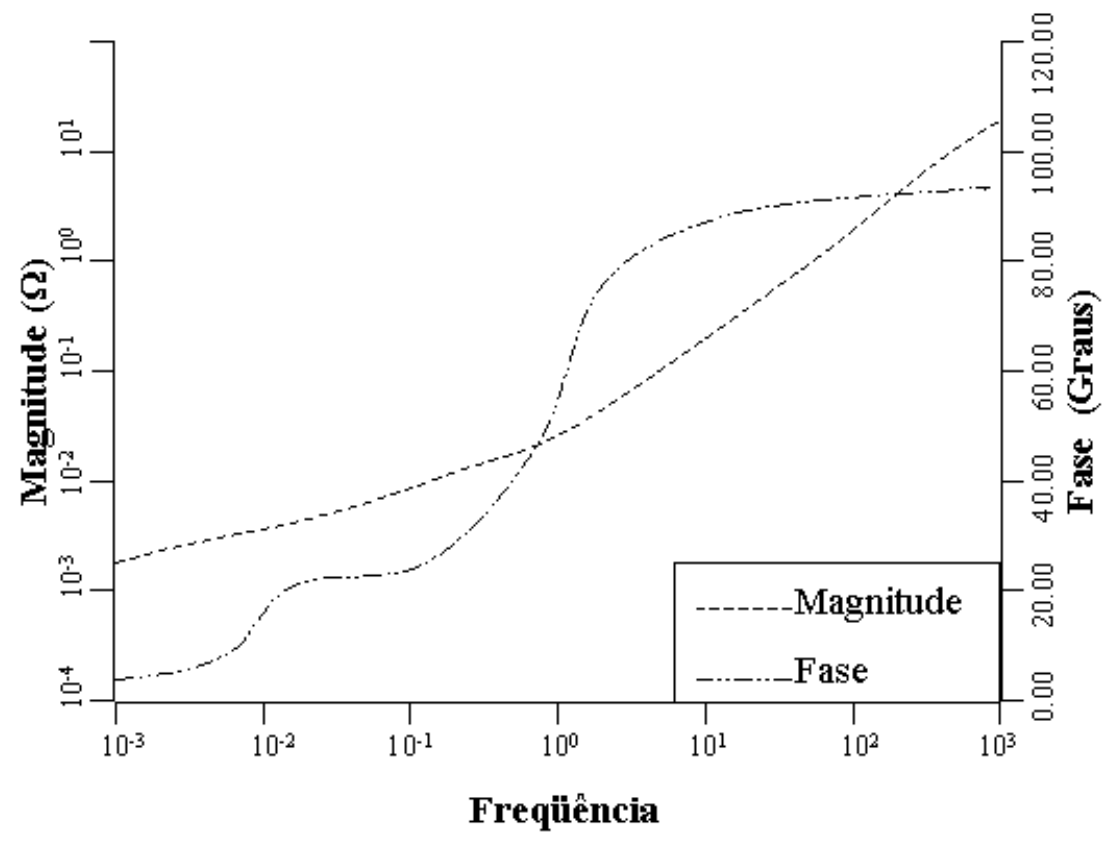

Figura 2.12: Impedância em eixo direto de $Z_{d}$ em função da freqüência.

Posicionamento do rotor para o teste de eixo em quadratura: Conectar o amplificador de potência nas fases "a"e "b"como mostra a figura (2.13). Ajustar o oscilador de freqüência a $100 \mathrm{~Hz}$. Observar a tensão de campo induzida no osciloscópio e girar o rotor do gerador até que a tensão de campo induzida seja nula.

Cálculo de $L_{q}(s)$ : Conectar os sinais de $v_{\text {arm }}$ e $i_{\text {arm }}$ e calcular: 


$$
\begin{aligned}
Z_{\text {armq }}(s) & =\frac{\Delta v_{\text {arm }}(s)}{\Delta i_{\text {arm }}(s)} \\
L_{q}(s) & =\frac{Z_{q}(s)-R_{a}}{s}
\end{aligned}
$$

onde: $Z_{q}(s)=\frac{1}{2} Z_{\text {armq }}(s)$ (impedância operacional de eixo em quadratura).

O valor de $R_{a}$ obtido durante o teste de eixo direto pode ser verificado por intermédio deste teste, assim $R_{a}$ pode ser determinado por :

$$
R_{a}=\frac{1}{2}\left\{\lim _{s \rightarrow 0}\left[Z_{a r m q}(s)\right]\right\}
$$

Os parâmetros da máquina no eixo direto são obtidos usando as funções de transferência aproximadas $L_{d}(s)$ e sG(s). A função de transferência sG(s) é mais usada que $\mathrm{G}(\mathrm{s})$ porque pode ser medida ao mesmo tempo que $Z_{d}(s)$, com o campo em curto circuito. Os parâmetros no eixo 'q' são obtidos usando funções de transferência para $L_{q}(s)$. A ordem da função de transferência depende do número de circuitos do rotor considerados no respectivo eixo.

Como os testes são realizados a níveis de freqüência muito baixos, os resultados devem ser corrigidos de valores saturados a níveis não saturados. Isto é realizado fazendo um ajuste na reatância mútua em cada eixo.

Além do requisito de ajuste dos parâmetros em condições não saturadas, há outras duas limitações para este teste, ambas envolvem os efeitos rotacionais por exemplo, que os amortecedores podem não fazer bom contato durante o teste.

Outro inconveniente do teste em SSFR, é a necessidade de conhecer a indutância de dispersão do enrolamento do estator $L_{l}$ antes da realização do teste.

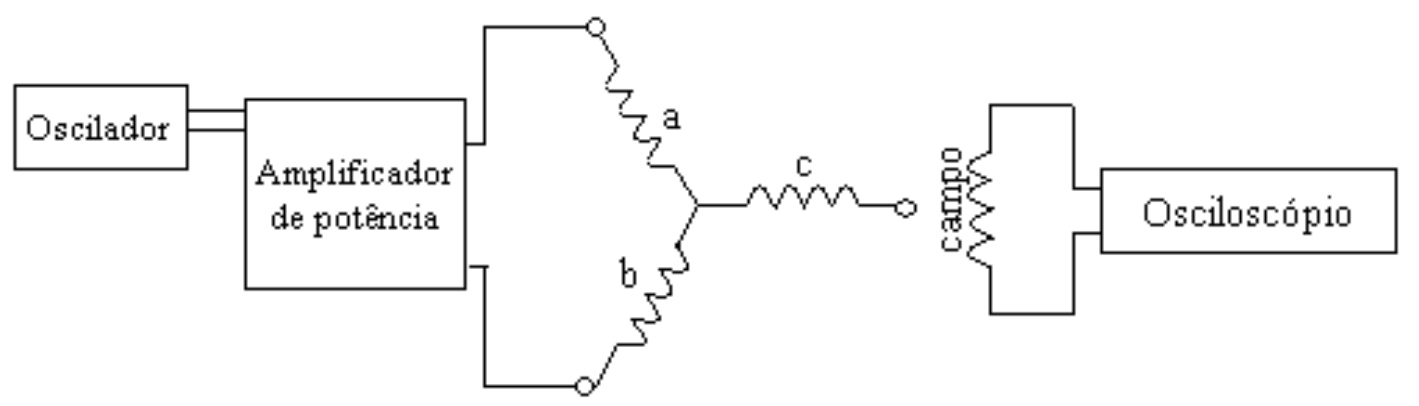

Figura 2.13: Teste para a medida de $\mathrm{Z}(\mathrm{q})$. 
Isto consegue-se por intermédio de testes adicionais da resposta em freqüência (tanto do circuito do rotor como do circuito de estator). Os estudos realizados por (Saunders, 1991) verificaram que existe dependência de $L_{l}$ com a freqüência, o que dificulta a estimação deste parâmetro.

No procedimento do teste em SSFR é necessário realizar ajustes de curvas (IEEE, 1995). Isto é conseguido por técnicas iterativas para igualar as constantes de tempo com a resposta medida e resolver um conjunto de equações não lineares e obter os parâmetros do circuito equivalente. A precisão dos parâmetros estimados dependerá dos valores iniciais escolhidos. Valores próximos dos valores reais podem ser obtidos através de uma técnica não iterativa (Henschel e Dommel, 1999). Os valores obtidos podem ser utilizados como valores iniciais por qualquer técnica de minimização 1 . Na mesma referência apresenta-se uma boa técnica para determinar a ordem do modelo do gerador no eixo direto e quadratura.

Para validar os resultados obtidos por meio dos testes em SSFR (Saunders, 1991) apresenta um procedimento para verificar se os parâmetros foram corretamente estimados. A análise é realizada através da separação da função de transferência em três componentes, e do resíduo da função de transferência. $\mathrm{Na}$ referida pesquisa também são apresentados os limites para a faixa de freqüência desse estudo.

Experiências de análise de testes em SSFR para diferentes máquinas síncronas de pólos salientes são relatadas em (Dandeno et al., 1999). Neste estudo mostrouse que em freqüências muito baixas, a impedância operacional é muito sensível às variações da resistência de armadura.

Entre as vantagens do teste SSFR podem-se citar: são seguros e boas aproximações dos parâmetros do eixo direto e quadratura são obtidos. O método apresenta alguns problemas por não considerar os efeitos rotacionais (indutância de dispersão), e alguns ajustes devem ser feitos em relação à saturação. Muitas propostas para melhorar a precisão dos parâmetros determinados por este teste

\footnotetext{
${ }^{1}$ Exemplo Ajuste de curvas como o mostrado em (IEEE, 2002)
} 
são relatadas na literatura (de Mello e Hannett, 1998), (Dandeno et al., 1999), (Saunders, 1991), (Henschel e Dommel, 1999). O IEEE (IEEE, 1995) padronizou o procedimento para a estimação dos parâmetros através dos testes em SSFR. A precisão dos parâmetros estimados depende também da precisão dos instrumentos utilizados, que devem ser capazes de medir módulo e ângulo de sinais alternados com freqüências por vezes inferiores a $0.01 \mathrm{~Hz}$. Além disso, o tratamento dos dados obtidos deve ser feito através de técnicas avançadas de ajuste de curvas.

\subsubsection{Resposta em Freqüência em circuito aberto (OCFR)}

Os testes OCFR (do inglês, Open Circuit Frequency Response) são realizados a velocidade nominal com a máquina conectada ou não ao sistema, são mais difíceis e mais caros de se realizar, entretanto fornecem informações adicionais se comparados ao teste em SSFR (efeitos rotacionais) (Chow et al., 1999), (Henschel e Dommel, 1999).

Este teste permite a confirmação de alguns dados do teste SSFR, mas só no eixo direto no meio do intervalo de freqüência. Para isto o gerador é operado em circuito aberto a um nível de tensão reduzido. O circuito de campo é excitado em várias freqüências, e a resposta do estator a variações no campo é medida. Este teste consegue indicar alguns efeitos rotacionais e é realizado para diferentes tensões para examinar os efeitos da saturação (Kundur, 1994).

\subsubsection{Resposta de Freqüência 'on-line' (OLFR)}

Nos testes OLFR (do inglês, On Line Frequency Response), a máquina é testada em condições próximas a carga nominal (ou inferior) e preferencialmente com alta impedância do sistema. A condição de alta impedância admite que o sistema esteja com baixa carga (pouco consumo de potência) e evitando que pequenas perturbações não façam o sistema ficar instável. Os componentes do sistema são analisados em dois eixos assim como no teste de SSFR. O intervalo de freqüência é mais limitado do que no teste SSFR, e permite verificar os efeitos rotacionais. A condição de alta impedância é uma desvantagem pois é fácil conseguir essa 
condição. Além disso a amplitude do sinal de saída é muito pequena e requer-se de bastante tempo para realizar o teste (Kundur, 1994).

\subsubsection{Outras técnicas}

Além das metodologias descritas anteriormente, existem outras que não foram incluídas por terem características particulares como, por exemplo, a apresentada em (Weeber, 1997) na qual, através de uma representação da máquina síncrona por elementos finitos, foram realizadas simulações numéricas para a determinação dos parâmetros de uma máquina síncrona. O emprego desta metodologia de estimação é importante sobretudo na fase de projeto, permitindo a concepção de sistemas de controle e a predição do comportamento da máquina. A precisão dos resultados está limitada aos recursos computacionais e à modelagem utilizada para representar os materiais magnéticos.

A metodologia de sensibilidade da trajetória também não foi apresentada neste capítulo, pois será estudada em detalhe no próximo capítulo. Dentre as características importantes desta metodologia é a possibilidade de realização de testes 'on-line' e por permitir uma representação completa das não linearidades da máquina síncrona (por exemplo a saturação) e do sistema de excitação (por exemplo os limites do sistema de excitação).

\subsection{Quadro Comparativo}

Na figura 2.14 apresenta-se um resumo geral das metodologias indicando algumas das principais vantagens e desvantagens. Tomando em consideração o número de publicações, tanto os testes no domínio do tempo como no domínio da freqüência são igualmente aceitos pela comunidade científica. Muitos pesquisadores sugerem que, uma vez determinados os parâmetros num determinado domínio, seja realizado testes no outro domínio como maneira de verificação dos resultados. 


\begin{tabular}{|c|c|c|c|}
\hline \multicolumn{2}{|c|}{ Mebdologia } & \multirow{2}{*}{$\begin{array}{l}\text { Algumas } \\
\text { vantagens }\end{array}$} & \multirow[t]{2}{*}{ Algumas desvantagens } \\
\hline Teste & Técrica utilizada & & \\
\hline \multirow[t]{2}{*}{$\begin{array}{l}\text { Curto circuito } \\
\text { trifásioo }\end{array}$} & $\begin{array}{c}\text { Utiliza uma } \\
\text { análise gráfica }\end{array}$ & $\begin{array}{l}\text { 1) Sirmples para ser } \\
\text { realizado }\end{array}$ & $\begin{array}{l}\text { 1) A máquina síncroma é submetida a } \\
\text { esfoŗo mecầico } \\
\text { 2) Hão oonsegue estimar os parâmetros } \\
\text { do eixo q }\end{array}$ \\
\hline & $\begin{array}{l}\text { Aproximação } \\
\text { Linear por } \\
\text { mínimos } \\
\text { quadrados }\end{array}$ & $\begin{array}{l}\text { 1) Precisão dos dados } \\
\text { obtidos }\end{array}$ & $\begin{array}{l}\text { 1)Os mesmos incorveriertes que o } \\
\text { método tradicional de curto circuito }\end{array}$ \\
\hline $\begin{array}{l}\text { Inchusão de } \\
\text { perturbaçōes } \\
\text { (PRBS) }\end{array}$ & $\begin{array}{l}\text { Qualquer técrica } \\
\text { de mimimização } \\
\text { de eno }\end{array}$ & 1) Teste 'on-line' & $\begin{array}{l}\text { 1) Irqurecisão dos vabres calculados de } \\
\text { alguns parâmetros. }\end{array}$ \\
\hline $\begin{array}{l}\text { Rejeição de } \\
\text { cargas }\end{array}$ & $\begin{array}{l}\text { Qualquer técrica } \\
\text { de mimimização } \\
\text { de eno }\end{array}$ & $\begin{array}{l}\text { 1) É possível deteminar } \\
\text { os parâmetros do eino d } \\
\text { e eixo } \mathrm{q}\end{array}$ & $\begin{array}{l}\text { 1) Dificuldade para atingir as condiçoses } \\
\text { de operação para realizar a rejeiçã } \\
\text { 2) Requer város aciomamentos do } \\
\text { disuntor } \\
\text { 3) Tem dificuldades para obter } \\
\text { parâmetros nõo saturados }\end{array}$ \\
\hline $\begin{array}{l}\text { SSFR (Resposta } \\
\text { em frequiência } \\
\text { com rotor } \\
\text { parado) }\end{array}$ & $\begin{array}{l}\text { Ajuste de curvas } \\
\text { como, por } \\
\text { exempb, ajuste } \\
\text { de curvas }\end{array}$ & $\begin{array}{l}\text { 1) BaixaProbabilidade } \\
\text { de risco para a máquina } \\
\text { 2) Pode deteminaros } \\
\text { parâmetros no eino } \\
\text { direto e em quadratura }\end{array}$ & $\begin{array}{l}\text { 1) É necessário oonhecer a indutância de } \\
\text { dispersão e a ordem do modelo do } \\
\text { gerador artes de realizar o teste } \\
\text { 2) Precisa de um tratamerto especial } \\
\text { para inchiro efeito da saturaçăo nos } \\
\text { parâmetros estimados }\end{array}$ \\
\hline $\begin{array}{l}\text { OCFR (Resposta } \\
\text { em Frequîncia } \\
\text { em Circuito } \\
\text { Aberto) }\end{array}$ & Análise gráfica & $\begin{array}{l}\text { 1) Fomece alguma } \\
\text { infomação sobre os } \\
\text { efeitos rotaciomais }\end{array}$ & $\begin{array}{l}\text { 1) Teste apenas realizado para } \\
\text { verificação de alguns dados do teste } \\
\text { SSFR. }\end{array}$ \\
\hline $\begin{array}{l}\text { OLFR(Resposta } \\
\text { em Frequiência } \\
\text { 'on-line') }\end{array}$ & Análise gráfica & $\begin{array}{l}\text { 1) Fomece infomação } \\
\text { sobre os efeitos } \\
\text { rotacionais mo ponto de } \\
\text { operação }\end{array}$ & $\begin{array}{l}\text { 1) Precisa alta irmedância de carga. } \\
\text { 2) A amplitude do sinal de saída é routo } \\
\text { pequena } \\
\text { 3) Precisa bastarte tempo para realizar a } \\
\text { teste }\end{array}$ \\
\hline \multirow[t]{2}{*}{ Outras técnicas } & $\begin{array}{l}\text { Representação } \\
\text { do gerador por } \\
\text { Elemertos } \\
\text { Finitos }\end{array}$ & $\begin{array}{l}\text { 1) Utilizado na fase de } \\
\text { projeto da máquina }\end{array}$ & $\begin{array}{l}\text { 1) É apenas una representação } \\
\text { computaciomal para uma máquina }\end{array}$ \\
\hline & $\begin{array}{l}\text { Sersibilidade da } \\
\text { trajetória } \\
\text { (a ser amalisada } \\
\text { nos próximos } \\
\text { capinubs) }\end{array}$ & $\begin{array}{l}\text { 1) Pode ser } \\
\text { acondicionado para ser } \\
\text { realizado 'on-line'. } \\
\text { 2) Pemite una } \\
\text { representação detallhada } \\
\text { de sistemas não lineares }\end{array}$ & $\begin{array}{l}\text { 1) A preserta algumas dificuldades mo } \\
\text { momento de estimaçăo de vários } \\
\text { parâmetros. }\end{array}$ \\
\hline
\end{tabular}

Figura 2.14: Comparação de algumas metodologias de estimação de parâmetros. 


\section{Capítulo 3}

\section{Estimação de parâmetros \\ baseada na técnica de sensibilidade de trajetória}

\subsection{Introdução}

Neste capítulo apresenta-se a metodologia de estimação de parâmetros baseada na técnica de sensibilidade de trajetória para sistemas lineares e não lineares. Com o intuito de entender a metodologia, analisa-se a estimação de dois parâmetros de um pequeno sistema (sistema massa-mola).

A variação das soluções das equações diferenciais pela variação de seus parâmetros são conhecidas como funções de sensibilidade de trajetória. O estudo das funções de sensibilidade está relacionado com a necessidade de conhecer a dependência da solução de equações diferenciais em relação a seus parâmetros. Existem poucos relatos da teoria de sensibilidade, e mais especificamente de sensibilidade de trajetória para sistemas dinâmicos não lineares. Os livros (Cruz, 1972) e (Tomovic, 1963) apresentam algumas aplicações da teoria de sensibilidade para controle de sistemas utilizando modelos lineares. 


\subsection{Funções de Sensibilidade de Trajetória}

Seja $y(t, \alpha)$ o vetor solução, de uma equação diferencial ordinária que depende do parâmetro $\alpha$. A variação desta solução devido à variação do parâmetro, $d \alpha$, é aproximada por:

$$
\delta y(t, \alpha) \simeq \sum_{i}\left(\frac{\partial y}{\partial \alpha_{i}}\right)_{\alpha=\alpha_{n}} \delta \alpha_{i}
$$

As derivadas parciais

$$
\frac{\partial y}{\partial \alpha_{i}} \doteq \lambda^{i}
$$

são chamadas de funções de sensibilidade de trajetórias e descrevem os efeitos da variação dos parâmetros na solução da equação diferencial. Se a variação de um parâmetro altera significativamente a solução da equação diferencial então diz-se que a resposta do sistema é muito sensível à variação deste parâmetro. A estimação dos parâmetros é influenciada significativamente pela sensibilidade da saída como será mostrado no capítulo 6 (em geral parâmetros com maior sensibilidade convergem mais facilmente).

No exemplo a seguir, mostra-se a interpretação das funções de sensibilidades de trajetória.

Exemplo 3.1

Seja a equação diferencial descrita por:

$$
\frac{d^{3} y}{d t^{3}}+a_{2} \frac{d^{2} y}{d t^{2}}+a_{1} \frac{d y}{d t}+a_{o} y=u(t)
$$

sendo onde $\mathrm{u}(\mathrm{t})$ um degrau unitário.

Os parâmetros nominais são: $a_{2}=5.6 \quad a_{1}=16.2 \quad a_{0}=18$, com condições iniciais: $y(0)=0, \quad \frac{d y}{d t}(0)=0$.

A equação 3.3 é colocada na forma de espaço de estados por meio da seguinte transformação.

$$
\begin{aligned}
y & =x_{1} \\
\dot{x}_{1} & =x_{2} \\
\dot{x}_{2} & =x_{3} \\
\dot{x}_{3} & =-a_{o} x_{1}-a_{1} x_{2}-a_{2} x_{3}+u
\end{aligned}
$$


Derivando-se a equação (3.4) em relação ao parâmetro $a_{1}$ obtém-se a sensibilidade em relação a $a_{1}$ :

$$
\begin{aligned}
& \dot{\lambda}_{1}^{1}=\lambda_{2}^{1} \\
& \dot{\lambda}_{2}^{1}=\lambda_{3}^{1} \\
& \dot{\lambda}_{3}^{1}=-a_{o} \lambda_{1}^{1}-a_{1} \lambda_{2}^{1}-a_{2} \lambda_{3}^{1}-x_{2}
\end{aligned}
$$

sendo: $\lambda_{1}^{1}=\frac{\partial x_{1}}{\partial a_{1}}, \lambda_{2}^{1}=\frac{\partial x_{2}}{\partial a_{1}}, \lambda_{3}^{1}=\frac{\partial x_{3}}{\partial a_{1}}$.

A sensibilidade da saída em relação ao parâmetro $a_{1}$ pode ser obtida integrando-se as equações (3.5) em função do tempo e calculando-se:

$$
\frac{\partial y}{\partial a_{1}}=\lambda_{1}^{1}
$$

De maneira similar, podem ser encontradas as equações para as funções de sensibilidade da trajetória em relação aos parâmetros $a_{2}$ e $a_{o}$. A resposta da saída 'y' e as sensibilidades $\frac{\partial y}{\partial a_{2}}, \frac{\partial y}{\partial a_{1}}, \frac{\partial y}{\partial a_{o}}$, são mostradas na figura 3.1.

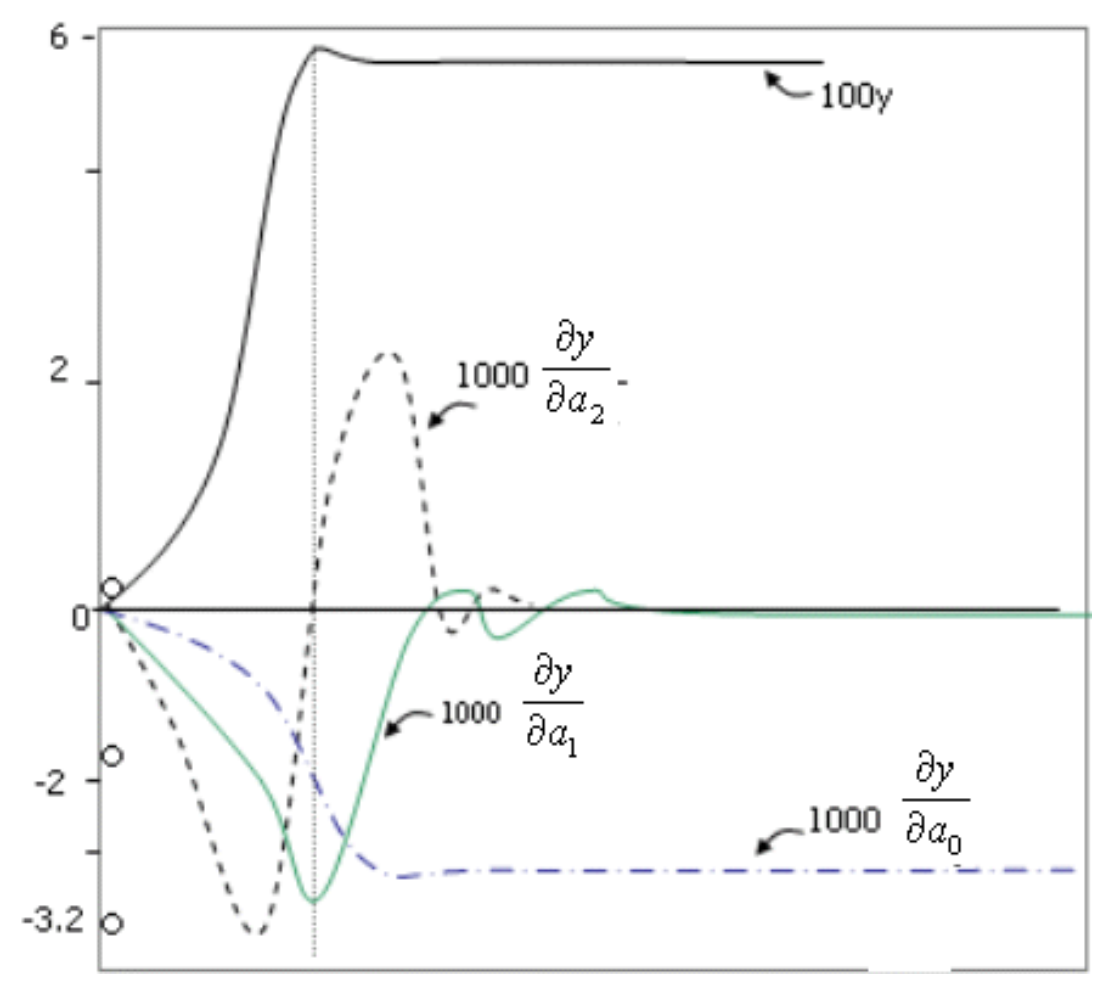

Figura 3.1: Saída e funções de sensibilidade para o exemplo 1.

As curvas foram representadas em diferentes escalas para melhor visualização dos resultados. Nessa figura observa-se que a curva $\frac{\partial y}{\partial a_{o}}$, indica que $a_{o}$ afeta 
principalmente o valor em regime permanente (valor final) da resposta, tendo pouco efeito no tempo de subida. A função de sensibilidade $\frac{\partial y}{\partial a_{1}}$ tem seu valor máximo quando ocorre o máximo sobrepasso (valor máximo), indicando que $a_{1}$ é o parâmetro que mais afeta o máximo sobrepasso da saída.

A variação de y em qualquer tempo pela variação de quaisquer parâmetros do sistema pode ser calculada pela equação (3.1) e é dada por

$$
\Delta y=\frac{\partial y}{\partial a_{o}} \Delta a_{o}+\frac{\partial y}{\partial a_{1}} \Delta a_{1}+\frac{\partial y}{\partial a_{2}} \Delta a_{2}
$$

As funções de sensibilidade para sistemas lineares invariantes no tempo podem ser obtidas, em princípio, resolvendo-se as equações lineares com parâmetros literais e diferenciando o resultado. Este procedimento é complicado para sistemas de baixa ordem e quase sempre impossível para sistemas variantes no tempo e sistemas não lineares, pois nem sempre é possível expressar a solução em termos de funções analíticas. Para contornar este problema são obtidas equações diferenciais para as funções sensibilidade de trajetória e a solução é obtida através de métodos numéricos. Na próxima seção, obtêm-se as funções de sensibilidade de trajetória para sistemas lineares invariante no tempo.

\subsubsection{Funções de Sensibilidade de Trajetória aplicadas a um sistema linear invariante no tempo (LTI)}

As funções de Sensibilidade de trajetórias podem ser obtidas para um sistema linear invariante no tempo (LTI). Suponha que o sistema LTI com parâmetro $\alpha$ é descrito por:

$$
\begin{aligned}
\frac{d}{d t} x(t, \alpha) & =\quad A(\alpha) x(t, \alpha)+B(\alpha) u(t) \\
y & =\quad C(\alpha) x(t, \alpha)
\end{aligned}
$$

com condição inicial $x(0, \alpha)=x_{o}$

Diferenciando a primeira equação de (3.8) em relação ao parâmetro $\alpha$, e aplicando a regra da cadeia temos:

$$
\dot{\lambda}^{i}=A(\alpha) \lambda^{i}(t, \alpha)+\frac{\partial A(\alpha)}{\partial \alpha_{i}} x(t, \alpha)+\frac{\partial B(\alpha)}{\partial \alpha} u(t)
$$


onde: $\lambda^{i}=\frac{\partial x}{\partial \alpha_{i}}$

Os coeficientes das matrizes $\mathrm{A}, \frac{\partial A}{\partial \alpha_{i}}$, $\frac{\partial B}{\partial \alpha_{i}}$, são calculados para $\alpha=\alpha_{n}$ (valor nominal), segundo a expressão da equação (3.1), para produzir as funções de sensibilidade para pequenas variações nas vizinhanças de $\alpha=\alpha_{n}$.

Diferenciando-se a saída, similarmente ao caso anterior, obtém-se:

$$
\frac{\partial y}{\partial \alpha}=\frac{\partial C(\alpha)}{\partial \alpha} x(t, \alpha)+C(\alpha) \dot{\lambda}(t, \alpha)
$$

A condição inicial para a equação 3.9 pode ser encontrada fazendo-se:

$$
\left.\lambda_{o}^{i} \equiv \lambda^{i}(t, \alpha)\right|_{t=o}=\left.\frac{\partial x(t, \alpha)}{\partial \alpha_{i}}\right|_{t=o}
$$

As equações (3.9) e (3.10) definem o modelo de sensibilidade.

\subsubsection{Funções de Sensibilidade de Trajetória para modelos não lineares}

Considere o sistema não linear modelado por:

$$
\begin{aligned}
\frac{d}{d t} x(t) & =f(x(t), p, u(t)) \\
y(t) & =g(x(t), p, u(t))
\end{aligned}
$$

onde 'x' é o vetor de variáveis de estado, 'y' é o vetor de saídas medidas, 'u' é o vetor entradas manipuladas e 'p' é o vetor de parâmetros do modelo a ser identificado. As funções 'f' e 'g' são funções não lineares contínuas e Lipschitzianas de $\mathrm{x}, \mathrm{p}$, e $\mathrm{u}$, as quais podem ou não ser diferenciáveis.

Considere $p_{i}$ a i-ésima componente de $\mathrm{p}$ e que as funções $\mathrm{f}$ e $\mathrm{g}$ sejam diferenciáveis com respeito a $p_{i}$. As sensibilidades $\frac{\partial x(t)}{\partial p_{i}}$ e $\frac{\partial y(t)}{\partial p_{i}}$ dos estados $x(t)$ e da saída $y(t)$ são calculadas respectivamente como:

$$
\begin{aligned}
\frac{d}{d t} \frac{\partial x(t)}{\partial p_{i}} & =\frac{\partial f(x(t), p, u(t))}{\partial x} \cdot \frac{\partial x(t)}{\partial p_{i}}+\frac{\partial f(x(t), p, u(t))}{\partial p_{i}} \\
\frac{\partial y(t)}{\partial p_{i}} & =\frac{\partial g(x(t), p, u(t))}{\partial x} \cdot \frac{\partial x(t)}{\partial p_{i}}+\frac{\partial g(x(t), p, u(t))}{\partial p_{i}}
\end{aligned}
$$

Se 'f' ou 'g' não são diferenciáveis com respeito a $p_{i}$, obtém-se valores aproximados para $\frac{\partial x(t)}{\partial p_{i}}$ e $\frac{\partial y(t)}{\partial p_{i}}$ da seguinte maneira: considere $p^{o}$ o vetor de parâmetro nominal 
de $\mathrm{p}, p^{1}$ é um vetor cuja i-ésima componente é $p_{i}^{1}=p_{i}^{o}+\Delta p_{i}$, onde $\Delta p_{i}$ é uma pequena perturbação. Seja $x_{o}(t)$ e $y_{o}(t)$ a resposta no tempo de $(3.12$ obtida usando $p_{o}$ e seja $x_{1}(t)$ e $y_{1}(t)$ a resposta no tempo obtida usando $p_{1}$. Desta forma as funções de sensibilidade de trajetórias podem ser aproximadas por:

$$
\begin{aligned}
\frac{\partial x(t)}{\partial p_{i}} & \approx \frac{x_{1}(t)-x_{o}(t)}{\Delta p_{i}} \\
\frac{\partial y(t)}{\partial p_{i}} & \approx \frac{y_{1}(t)-y_{o}(t)}{\Delta p_{i}}
\end{aligned}
$$

\subsection{Esquema de Aplicação da metodologia de sensibilidade da trajetória}

O esquema para a aplicação da metodologia de sensibilidade de trajetória para determinação dos parâmetros de um sistema qualquer é mostrado na figura 3.2

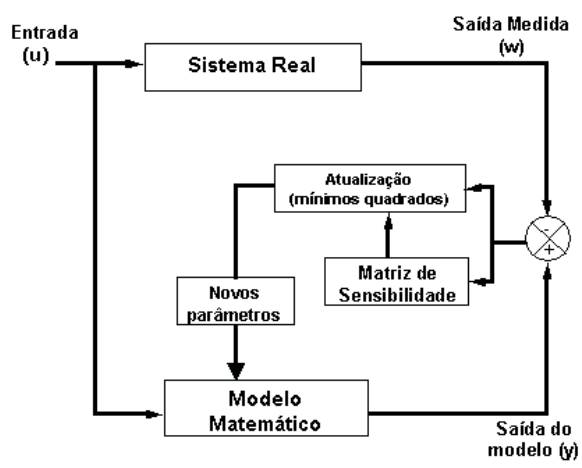

Figura 3.2: Metodologia de sensibilidade da trajetória para determinação de parâmetros.

\subsubsection{Processo de Minimização de Erro}

Este processo minimiza a diferença entre a saída do 'sistema real' com a saída do 'modelo matemático'. Para tal propósito, diferentes técnicas foram propostas na literatura (ver capítulo 2).

Utilizando o conceito de norma podemos entender melhor o processo de minimização de erro. O conceito de norma é uma generalização do conceito de 
magnitude e longitude.

Suponha que se queira minimizar a diferença entre as curvas da saída do 'modelo matemático' com as do 'sistema real' em todo o intervalo de tempo. A curva do 'modelo matemático' na figura 3.3(a), está distante da curva do 'sistema real' de maneira uniforme. Na figura 3.3(b), ambas as curvas estão mais próximas, entretanto em um pequeno intervalo a diferença entre as curvas é maior. Minimizar

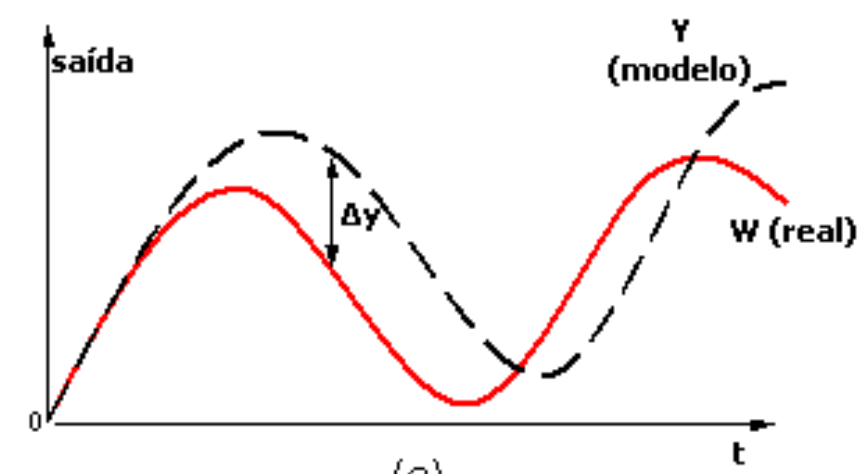

(a)

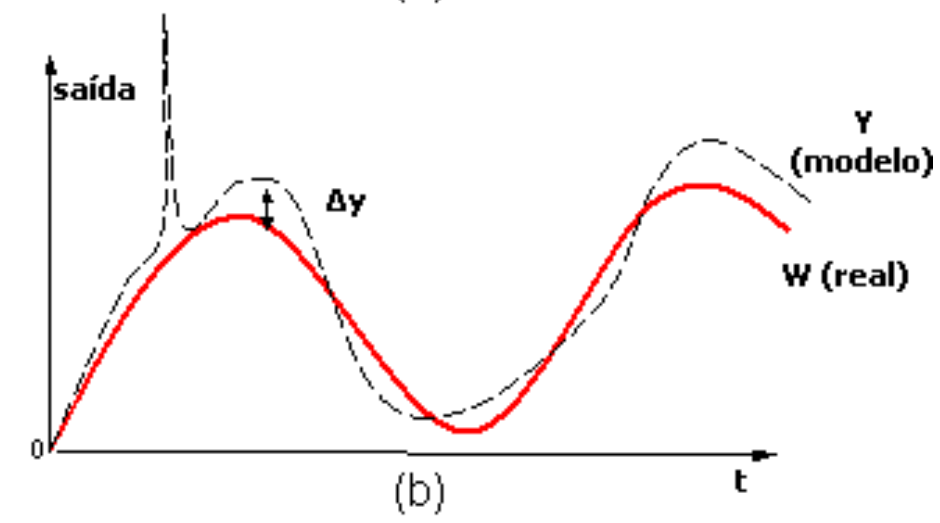

Figura 3.3: Comparação da saída do 'modelo matemático' e do 'sistema real'.

a diferença das curvas em valor absoluto, equivale a minimizar a norma 1 da diferença entre as duas funções.

$$
E(p)=\int_{0}^{T}\left|y_{i}(t)-w_{i}(t)\right| d t
$$

Minimizar a raiz quadrada da diferença, equivale a minimizar a norma 2 da diferença.

$$
E(p)=\int_{0}^{T} \sum_{0}^{n}\left(y_{i}(t)-w_{i}(t) d t\right)^{1 / 2}
$$


Minimizar a máxima diferença das saídas durante todo o intervalo de tempo equivale a minimizar a norma infinito da diferença.

$$
E(p)=\sup _{x \in[0, T]}\left|y_{i}(t)-w_{i}(t)\right|
$$

Diferentes normas levam o processo de minimização a diferentes soluções. Para o presente trabalho a norma 2 será utilizada, pela facilidade de sua representação e por ser diferenciável. O método de otimização baseado na norma 2 leva o nome de mínimos quadrados.

A formulação da minimização através do método de mínimos quadrados durante um intervalo de tempo $T_{o}$, é a seguinte:

$$
J(p)=\frac{1}{2} \int_{0}^{T_{o}}(y(t)-w(t))^{t}(y(t)-w(t)) d t
$$

Onde:

- $\mathrm{J}(\mathrm{p})$ : Funcional de erro a minimizar,

- y : saída do modelo,

- $w$ : saída do sistema real.

Neste caso, não se minimiza a raiz quadrada da integral, mas simplesmente a integral. O fator $\frac{1}{2}$ na equação (3.18) é apenas uma constante para simplificar os cálculos.

Para estimativas iniciais do vetor de parâmetros $p=p_{o}$, o problema de otimização pode ser resolvido calculando-se a derivada parcial de $\mathrm{J}(\mathrm{p})$ em relação a seus parâmetros e igualando-se esta expressão a zero,

$$
\frac{\partial J(p)}{\partial p}=\frac{1}{2} \int_{0}^{T_{0}}\left(\frac{\partial y}{\partial p}\right)^{t}(y-w) d t+\left.(y-w)^{t}\left(\frac{\partial y}{\partial p}\right) d t\right|_{p=p_{0}}
$$

Os dois termos desta integral são escalares e iguais, portanto pode-se simplificar esta expressão escrevendo-a como:

$$
\frac{\partial J(p)}{\partial p}=\int_{0}^{T_{0}}\left(\frac{\partial y}{\partial p}\right)^{t}(y-w) d t
$$


A equação 3.21 está em função do parâmetro p. Fazendo $G(p)=\frac{\partial J(p)}{\partial p}$ e expandindo em série de Taylor no ponto $p=p_{i}$ e tomando apenas o termo de primeira ordem tem-se:

$$
\begin{aligned}
& G(p)=G\left(p_{i}\right)+\frac{\partial G\left(p_{i}\right)}{\partial p} \Delta p+\ldots+=0 \\
& G(p)=G\left(p_{i}\right)+\frac{\partial G(p)}{\partial p} \triangle p=0
\end{aligned}
$$

substituindo-se $\frac{\partial G(p)}{\partial p}$ por $\Gamma$ na segunda parte da equação anterior tem-se:

$$
\Gamma \Delta p=-G\left(p_{i}\right)
$$

Isolando $\Delta p$ da equação 3.24 tem-se:

$$
\Delta p=-\Gamma^{-1} G\left(p_{i}\right)
$$

Os parâmetros são ajustados para a i-ésima iteração por:

$$
p_{(i+1)}=p_{(i)}+\Delta p_{(i+1)}
$$

A matriz $\Gamma$ pode ser calculada derivando-se a equação (3.21). Assim tem-se:

$$
\begin{aligned}
\Gamma & =\left.\frac{\partial}{\partial p} \int_{0}^{T_{0}}\left(\frac{\partial y}{\partial p}\right)^{t}(y-w) d t\right|_{p o} \\
\Gamma & =\int_{0}^{T_{0}}\left(\frac{\partial^{2} y}{\partial p^{2}}\right)^{t}(y-w) d t+\left.\left(\frac{\partial y}{\partial p}\right)^{t} \frac{\partial y}{\partial p} d t\right|_{p o}
\end{aligned}
$$

Desprezando o termo de segunda ordem (de acordo com a linearização feita na série de Taylor) obtém-se:

$$
\Gamma=\left.\int_{0}^{T_{0}}\left(\frac{\partial y}{\partial p}\right)^{t}\left(\frac{\partial y}{\partial p}\right) d t\right|_{p o}
$$

As funções de sensibilidade $\frac{\partial y}{\partial p}$ são calculadas pela equação 3.13. Finalmente, considerando que os dados disponíveis são amostras em intervalos de tempo discreto, as integrais das equações acima transformam-se em somatórios.

O procedimento é realizado sucessivamente até a convergência dos parâmetros como mostra a figura 3.4 . 


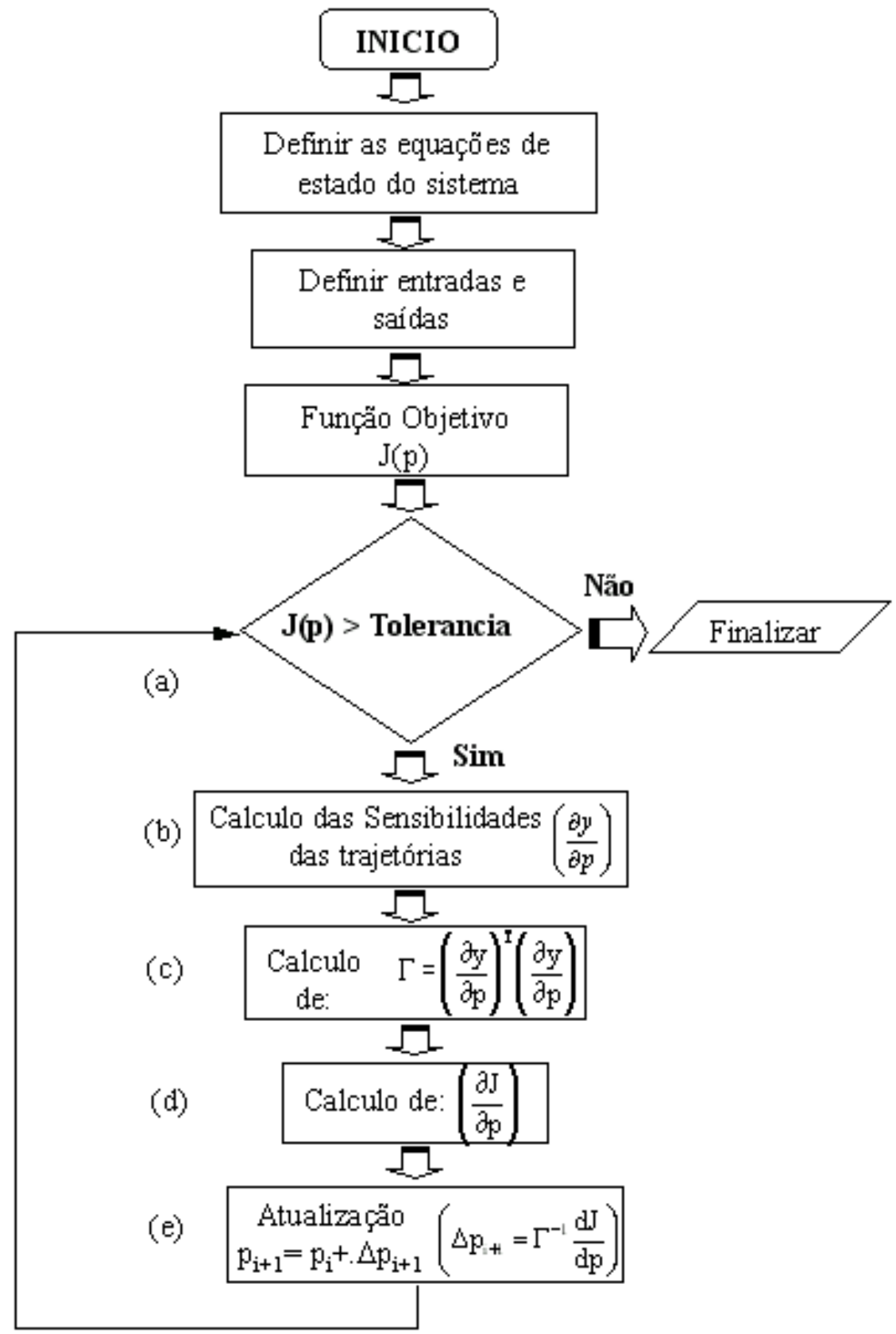

Figura 3.4: Diagrama esquemático da metodologia de Sensibilidade da trajetória. 


\subsubsection{Testes preliminares}

Com o intuito de estudar analiticamente a metodologia de sensibilidade de trajetória, são realizados dois testes preliminares para determinar parâmetros de um pequeno sistema (sistema massa-mola). Estes testes serão utilizados como base para aplicação da metodologia em sistemas mais complexos.

\section{a)Estimação de 1 parâmetro}

Seja o sistema massa-mola mostrado na figura 3.5. A equação que descreve a

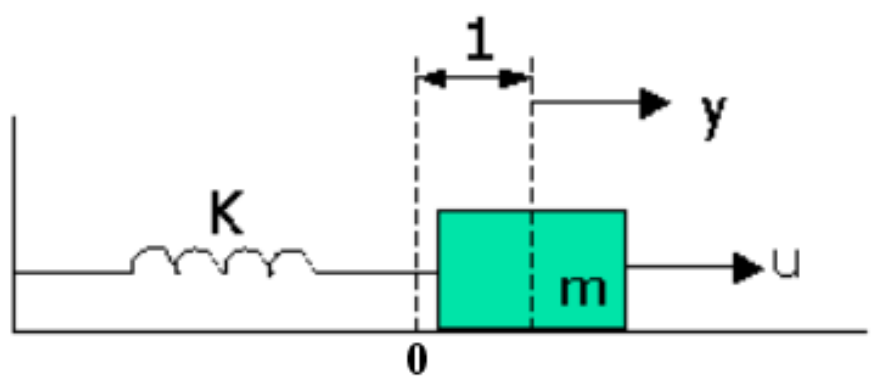

Figura 3.5: Representação física do sistema massa mola.

dinâmica do sistema é a seguinte:

$$
m \ddot{y}=u-k \cdot y
$$

A equação (3.30) é representada por um sistema de equações de primeira ordem fazendo-se a seguinte mudança de variável:

$$
\begin{aligned}
& x_{1}=y \\
& x_{2}=\dot{y}
\end{aligned}
$$

Obtém-se

$$
\begin{aligned}
& \dot{x}_{1}=x_{2} \\
& \dot{x}_{2}=\frac{u}{m}-\frac{k}{m} x_{1}
\end{aligned}
$$

Neste primeiro teste a relação k/m será nosso parâmetro a estimar. Vamos admitir que é possível medir a posição do corpo de massa 'm' e portanto a equação de saída deste sistema é $y=x_{1}$. Na figura 3.6 mostra-se a representação do 
esquema de estimação considerando o 'sistema real' e o 'modelo matemático'. Na referida figura ' $p$ ' denota o parâmetro verdadeiro e 'p' é o parâmetro do modelo matemático a ser estimado.

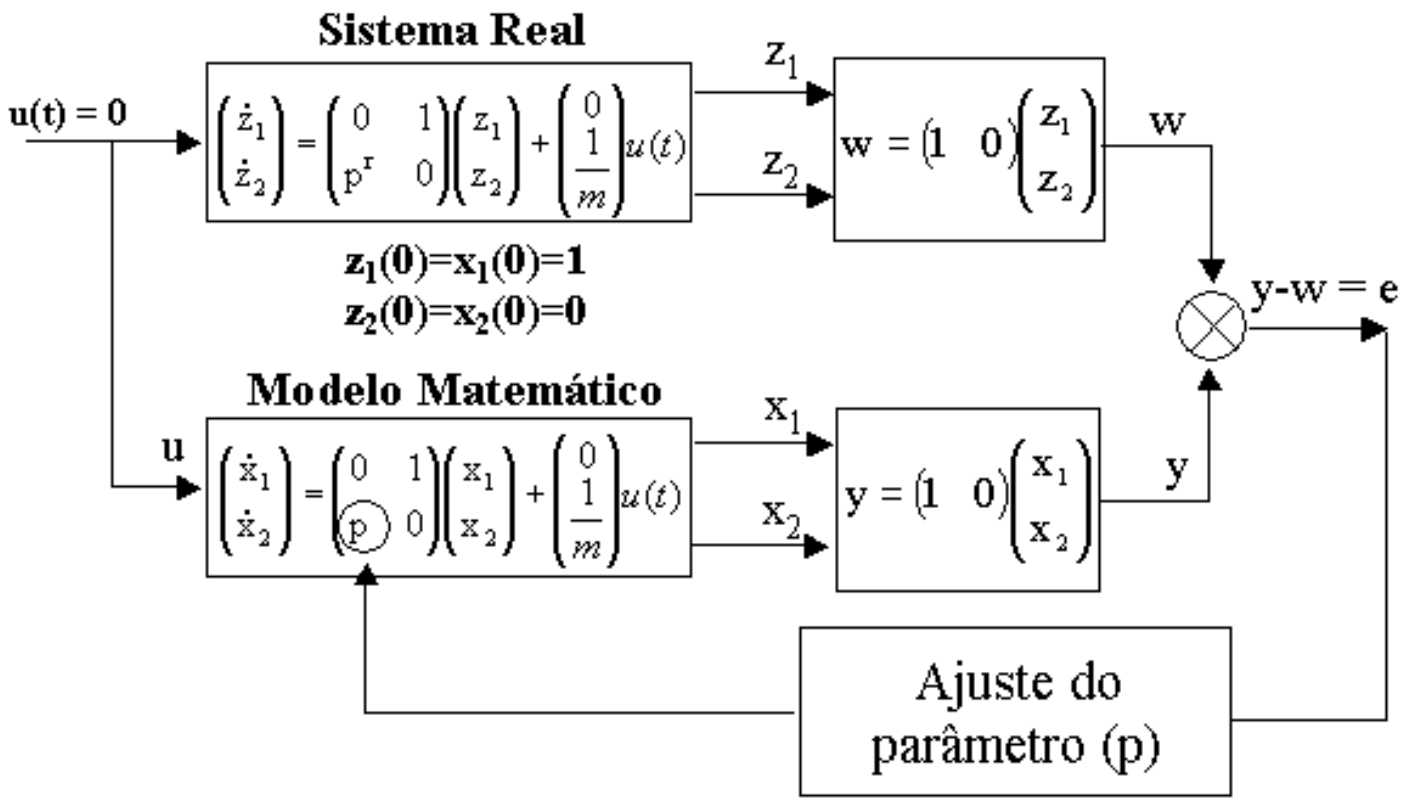

Figura 3.6: Diagrama esquemático para a estimação dos para o sistema massa-mola.

Existem duas formas de obter uma resposta dinâmica dos sistemas representados na figura 3.6. por intermédio de uma condição inicial diferente de zero, ou por uma entrada diferente de zero. Neste primeiro teste, considera-se que algumas das condições iniciais são diferentes de zero.A entrada $u$ é zero. As condições iniciais do teste são: $x_{1}(0)=z_{1}(0)=1 \quad x_{2}(0)=z_{2}(0)=0$.

O parâmetro inicial utilizado para o modelo matemático é $p=1.5$ e o parâmetro verdadeiro é $p^{r}=2$. As saídas, tanto do 'sistema real' quanto do 'modelo matemático', são mostradas na figura 3.6. As saídas foram obtidas explicitamente através de integração analítica dadas por:

$$
\begin{aligned}
& w(t)=\cos \left(\sqrt{p^{r}} t\right) \\
& y(t)=\cos (\sqrt{p} t)
\end{aligned}
$$

Procedendo segundo a metodologia da figura (3.4), tem-se:

- (a) Funcional de erro : $J(p)$ 


$$
\begin{aligned}
& J(p)=\frac{1}{2} \int_{0}^{T}(y-w)^{t}(y-w) d t \\
& J(p)=\frac{1}{2} \int_{0}^{T}\left(\cos (\sqrt{p} t)-\cos \left(\sqrt{p^{r}} t\right)\right)^{2} d t
\end{aligned}
$$

- (b) Função de sensibilidade : $\frac{\partial y}{\partial p}$

$$
\frac{\partial y}{\partial p}=-\frac{t}{2 \sqrt{p}} \operatorname{sen}(\sqrt{p} t)
$$

- (c)Cálculo de $\Gamma$ :

$$
\begin{aligned}
& \Gamma=\left.\int_{0}^{T} \frac{\partial y}{\partial p} \frac{\partial y}{\partial p} d t\right|_{p} \\
& \Gamma=\left.\int_{0}^{T}\left(\frac{-t \operatorname{sen}(\sqrt{p} t)}{2 \sqrt{p}}\right)^{2} d t\right|_{p} \\
& \Gamma=\frac{1}{4 p^{\frac{5}{4}}}\left(-p t^{2} \cos (\sqrt{p} t)+2 \cos (\sqrt{p} t)+\sqrt{p} t \operatorname{sen}(\sqrt{p} t)-2\right)
\end{aligned}
$$

- (d) Cálculo de $\frac{\partial J(p)}{\partial p}$ :

$$
\begin{aligned}
& \frac{\partial J(p)}{\partial p}=\int_{0}^{T} \frac{\partial y}{\partial p}^{t}(w-y) d t \\
& \frac{\partial J(p)}{\partial p}=\int_{0}^{T} \frac{-t \operatorname{sen}(\sqrt{p} t)}{2 \sqrt{p}}\left(\cos \left(p_{0} t\right)-\cos (\sqrt{p} t)\right) d t \\
& \frac{\partial J(p)}{\partial p}=\frac{-1}{2 \sqrt{p}}\left[\frac{1}{2(\sqrt{p}+2)^{2}}(\operatorname{sen}((\sqrt{p}+2)) T)-(\sqrt{p}+2) T \cos ((\sqrt{p}+2) T)+\right. \\
& \left.\frac{1}{2(\sqrt{p}-2)}(\operatorname{sen}((\sqrt{p}-2)) T)-(\sqrt{p}-2) T \cos ((\sqrt{p}-2) T)-\frac{1}{4 \sqrt{p}}(\cos (2 \sqrt{p} T)-1)\right]
\end{aligned}
$$

- (e) Cálculo de $\Delta_{p}$ :

$$
\Delta_{p(i+1)}=\Gamma^{-1} \frac{\partial J(p)}{\partial p}
$$

O parâmetro foi estimado após 43 iterações. Na figura 3.7 mostram-se as saídas antes e depois da estimação do parâmetro. O resumo dos resultados é mostrado
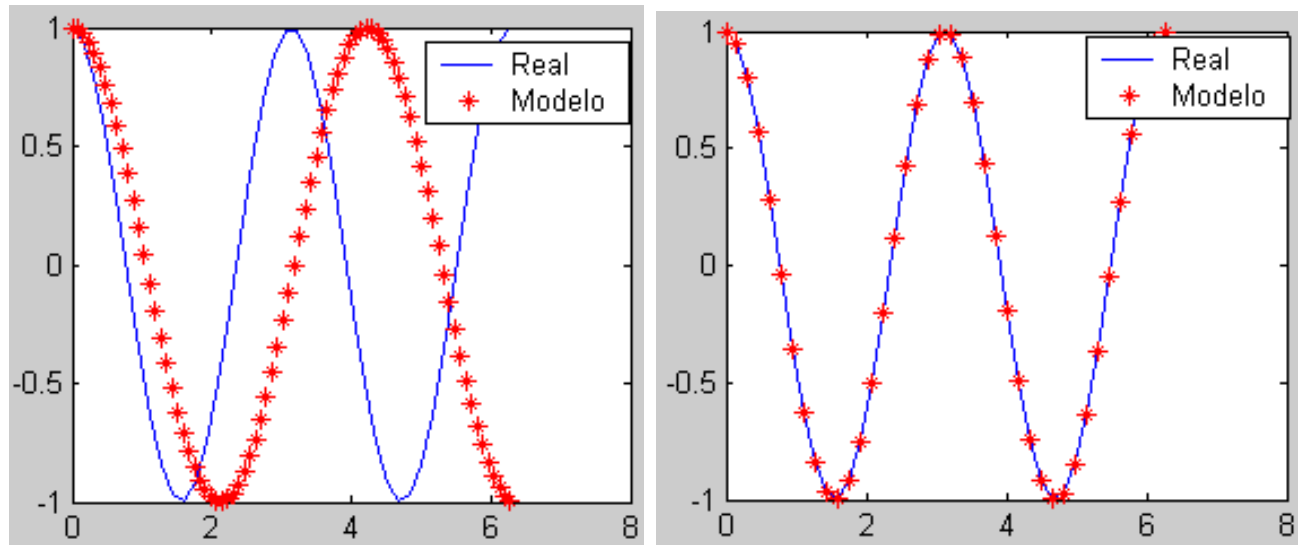

Figura 3.7: Saída inicial e final no teste de determinação de 1 parâmetro.

na tabela (3.1), o erro foi inferior a $3 \%$. 
Tabela 3.1: Teste para estimação de um parâmetro do sistema massa mola.

\begin{tabular}{|c|c|c|c|c|}
\hline parâmetro & V. Inicial & V. Final & V. Real & Erro (\%) \\
\hline $\mathrm{p}=\frac{k}{m}$ & 1.5 & 2.0496 & 2 & 2.48 \\
\hline
\end{tabular}

\section{b) Determinação de 2 parâmetros}

Um segundo teste foi realizado, mas agora para tentar determinar os dois parâmetros 'k' e 'm' simultaneamente. Inicialmente utilizou-se o mesmo modelo e as mesmas considerações do caso anterior (estado inicial diferente de zero) porém, não foi possível estimar os parâmetros porque as sensibilidades com respeito a 'k' e 'm' são linearmente dependentes. Isto pode ser verificado explorando-se a singularidade da matriz $\Gamma$.

O vetor de parâmetro é : $p=\left[\begin{array}{l}k \\ m\end{array}\right]$

A saída é a mesma do caso anterior :

$y(t)=\cos \left(\sqrt{\frac{k}{m}} t\right)$

A matriz de sensibilidade é calculada como:

$$
\begin{aligned}
& \frac{\partial y}{\partial p}=\left[\begin{array}{ll}
\frac{\partial y}{\partial k} & \frac{\partial y}{\partial m}
\end{array}\right] \\
& \frac{\partial y}{\partial p}=\left[\begin{array}{ll}
-\frac{t}{2 \sqrt{\frac{k}{m}}} \operatorname{sen}\left(\sqrt{\frac{k}{m}} t\right) & \frac{t k^{\frac{1}{2}}}{2 m^{\frac{3}{2}}} \operatorname{sen}\left(\sqrt{\frac{k}{m}} t\right)
\end{array}\right]
\end{aligned}
$$

Substituindo $\frac{t}{2} \operatorname{sen} \sqrt{\frac{k}{m}}$ por H, tem-se

$$
\frac{\partial y}{\partial p}=\left[\begin{array}{ll}
-\frac{H}{\sqrt{\frac{k}{m}}} & \frac{H k^{\frac{1}{2}}}{m^{\frac{3}{2}}}
\end{array}\right]
$$

A matriz $\Gamma$ é dada por:

$$
\begin{aligned}
\Gamma & =\left(\frac{\partial y}{\partial p}\right)^{t}\left(\frac{\partial y}{\partial p}\right) \\
\Gamma & =H^{2}\left(\begin{array}{cc}
\frac{1}{m k} & \frac{1}{m^{2}} \\
\frac{1}{m^{2}} & \frac{k}{m^{3}}
\end{array}\right)
\end{aligned}
$$


O determinante de $\Gamma$

$$
|\Gamma|=\frac{H^{2}}{m^{4}}-\frac{H^{2}}{m^{4}}=0
$$

Com isto verifica-se que é impossível através deste teste estimar os dois parâmetros simultaneamente. Entretanto, isto não significa que não exista um teste que permita a estimação simultânea destes parâmetros.

Para contornar o problema da singularidade da matriz $\Gamma$, um novo teste é aplicado. A resposta dinâmica neste caso será obtida através de uma entrada diferente de zero $(u=4)$. As condições iniciais serão iguais a zero. Os parâmetros a serem estimados são 'k' e 'm'. As equações que descrevem a dinâmica estão representadas pelo diagrama de blocos da figura (3.8).

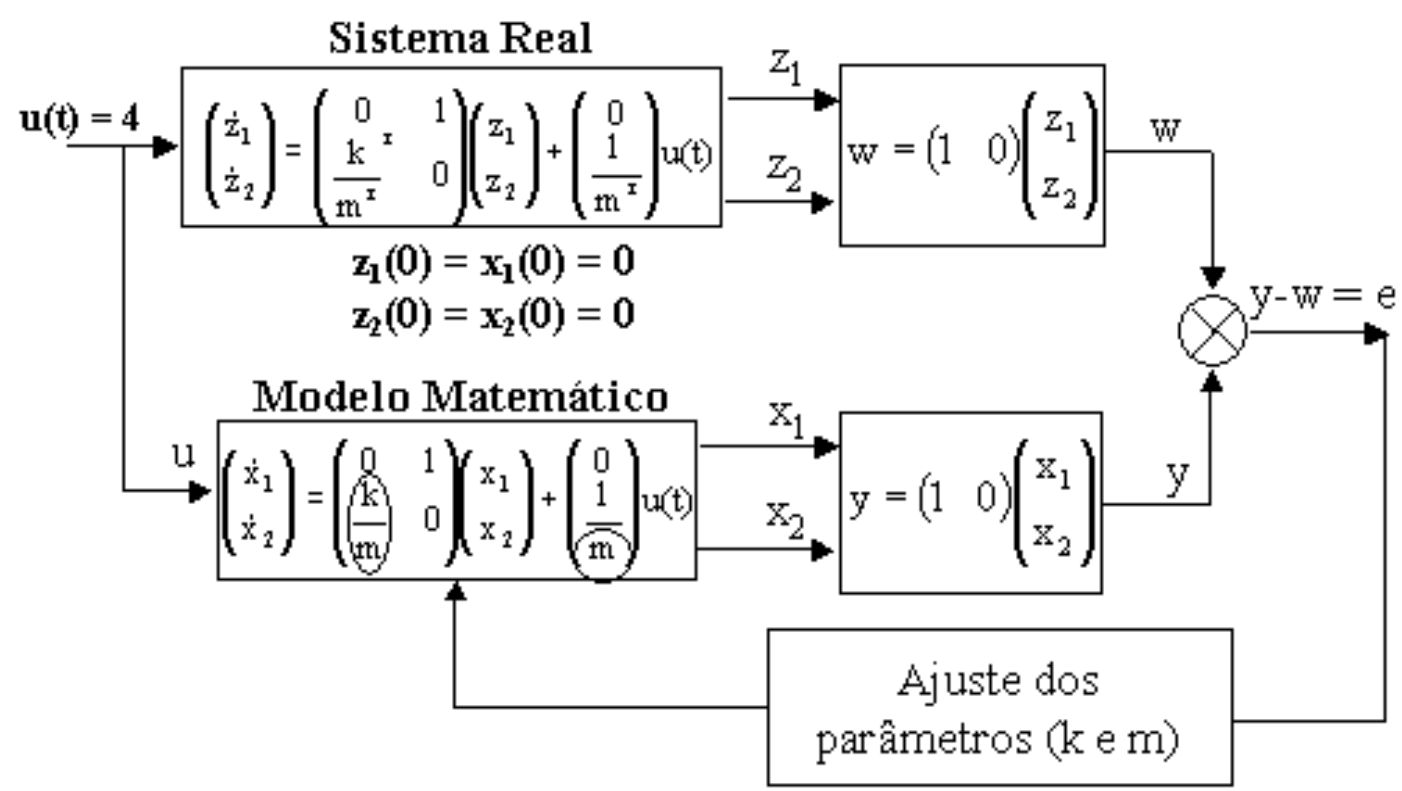

Figura 3.8: Entradas e saídas para estimação de 'k' e 'm' simultaneamente.

A solução analítica pode ser calculada através da formulação da variação das constantes :

$$
\begin{aligned}
& x(t)=e^{A t} x_{o}+\int_{0}^{t} e^{A(t-\tau)} B u(\tau) d \tau \\
& y(t)=C \int_{0}^{t} e^{A(t-\tau)} B u(\tau) d \tau
\end{aligned}
$$


Assim, a saída do modelo 'y' na forma analítica é dada pela seguinte equação: $y=\frac{u}{k}\left(1-\cos \left(\sqrt{\frac{k}{m}} T\right)\right)$

No caso do 'sistema real' a saída ( $w$ ) é dado pela expressão:

$w=\frac{u}{k}\left(1-\cos \left(\sqrt{\frac{k_{\text {real }}}{m_{\text {real }}}} T\right)\right)$

As funções de sensibilidade podem ser calculadas derivando-se as equações de estado em função dos parâmetros. Para isto definem-se:

$\lambda_{1}^{k}=\frac{\partial x_{1}}{\partial k}$

$\lambda_{2}^{k}=\frac{\partial x_{2}}{\partial k}$

$\lambda_{1}^{m}=\frac{\partial x_{1}}{\partial m}$

$\lambda_{2}^{m}=\frac{\partial x_{2}}{\partial m}$

Tem-se:

$$
\left(\begin{array}{c}
\dot{\lambda_{1}^{k}} \\
\dot{\lambda_{2}^{k}} \\
\dot{\lambda_{1}^{m}} \\
\dot{\lambda_{2}^{m}}
\end{array}\right)=\left(\begin{array}{cccc}
0 & 1 & 0 & 0 \\
-\frac{k}{m} & 0 & 0 & 0 \\
0 & 0 & 0 & 1 \\
0 & -\frac{k}{m} & 0 & 0
\end{array}\right)\left(\begin{array}{c}
\lambda_{1}^{k} \\
\lambda_{2}^{k} \\
\lambda_{1}^{m} \\
\lambda_{2}^{m}
\end{array}\right)+\left(\begin{array}{cc}
0 & 0 \\
\frac{-1}{m} & 0 \\
0 & 0 \\
\frac{1}{m^{2}} & 0
\end{array}\right)\left(\begin{array}{c}
\frac{u}{k}\left(1-\cos \left(\sqrt{\frac{k}{m}}\right) t\right) \\
0
\end{array}\right)+\left(\begin{array}{c}
0 \\
0 \\
0 \\
\frac{-1}{m^{2}}
\end{array}\right) u
$$

A solução analítica deste sistema são:

Funções de sensibilidades para o parâmetro 'k':

$$
\begin{aligned}
\lambda_{1}^{k} & =\frac{-u}{k m \sqrt{\frac{k}{m}}}\left(\frac{-1}{2 \sqrt{\frac{k}{m}}}\right)\left(-2+\operatorname{sen}\left(\sqrt{\frac{k}{m}} T\right) T \sqrt{\frac{k}{m}}+2 \cos \left(\sqrt{\frac{k}{m} T}\right)\right) \\
\lambda_{2}^{k} & =\frac{u}{2 k m \sqrt{\frac{k}{m}}}\left(-\operatorname{sen}\left(\sqrt{\frac{k}{m}} T\right)+\cos \left(\sqrt{\frac{k}{m}} T\right) T \sqrt{\frac{k}{m}}\right)
\end{aligned}
$$

Funções de sensibilidades para o parâmetro 'm':

$$
\begin{aligned}
\lambda_{1}^{m} & =\frac{u}{k m}\left[\left(\frac{1}{k}-1\right)\left(1-\cos \left(\sqrt{\frac{k}{m}}\right)\right)-\frac{\frac{k}{m}}{2} \operatorname{sen}\left(\sqrt{\frac{k}{m}} T\right) T\right] \\
\lambda_{2}^{m} & =\frac{u}{\sqrt{\frac{k}{m}} m^{2}}\left[\left(\frac{1}{k}-\frac{3}{2}\right) \operatorname{sen}\left(\sqrt{\frac{k}{m}} T\right)-\frac{\sqrt{\frac{k}{m}}}{2} \cos \left(\sqrt{\frac{k}{m}} T\right) T\right]
\end{aligned}
$$

A matriz $\Gamma$ é dada por:

$$
\Gamma=\left(\frac{\partial y}{\partial p}\right)^{t}\left(\frac{\partial y}{\partial p}\right)
$$


onde

$$
\frac{\partial y}{\partial p}=\left[\begin{array}{ll}
\frac{\partial y}{\partial k} & \frac{\partial y}{\partial m}
\end{array}\right]
$$

é a matriz de sensibilidade.

Utilizando o processo iterativo para minimizar o erro, encontram-se os resultados da figura 3.9, onde mostram-se as saídas do 'modelo matemático' assim como do sistema real antes e após da estimação dos parâmetros.
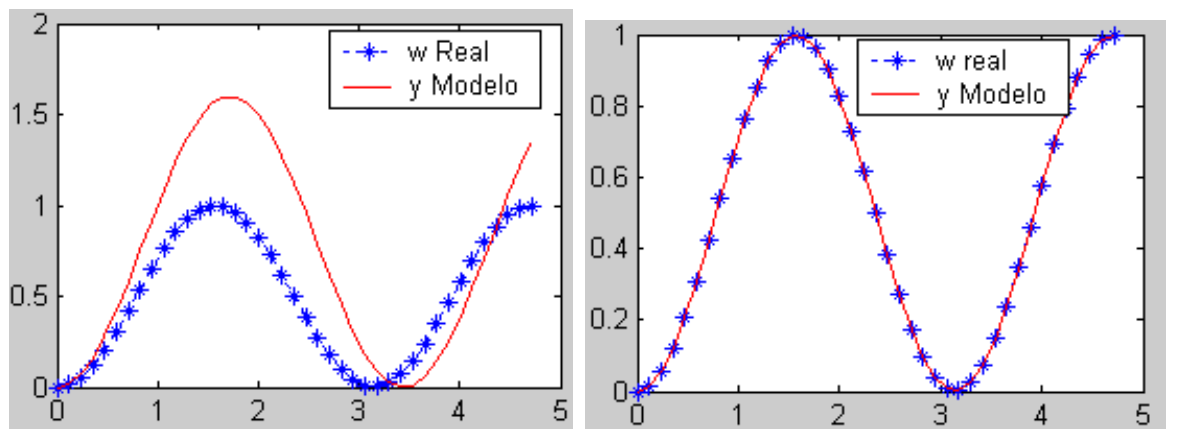

Figura 3.9: Saída do 'modelo matemático' e 'sistema real' antes e após a estimação dos dois parâmetros no sistema massa-mola.

Na tabela 3.2, mostram-se os valores dos parâmetros obtidos. Ambos os parâmetros (k e m) foram determinados com uma excelente precisão após 5 iterações.

Tabela 3.2: Teste para estimação de dois parâmetros do sistema massa-mola.

\begin{tabular}{|c|c|c|c|c|}
\hline parâmetro & V. Inicial & V. Final & V. Verdadeiro & Erro $(\%)$ \\
\hline $\mathrm{k}$ & 5 & 7.9999 & 8 & 0.001 \\
\hline $\mathrm{m}$ & 1.5 & 2.000 & 2 & 0 \\
\hline
\end{tabular}

Nestes primeiros testes, os parâmetros do sistema massa-mola foram satisfatoriamente determinados. No capítulo 5, aplica-se esta técnica para a estimação de parâmetros da máquina síncrona e do sistema de excitação. 


\section{Capítulo 4}

\section{Sincronização de sistemas aplicada à estimação de parâmetros}

\subsection{Introdução}

O processo de estimação de parâmetros a ser desenvolvido neste trabalho pode ser representado basicamente pelo diagrama esquemático da figura 4.1. Os blocos do 'Sistema Real' e 'Modelo Matemático' são parte do processo.

Tanto o bloco da 'Sistema Real' como o do 'Modelo Matemático' possuem a mesma entrada u, e ambas as saídas são comparadas. Em função do erro, uma estimativa do ajuste do parâmetro a ser estimado é obtida. A técnica é realizada sucessivamente até que ambas as saídas estejam muitos próximas.

Se os parâmetros iniciais do modelo matemático não forem suficientemente próximos dos parâmetros verdadeiros, o método pode não convergir. Este procedimento de estimação baseia-se no seguinte resultado clássico da teoria qualitativa de Equações Diferenciais Ordinárias (EDO's):

Teorema 4.1 Suponhamos que $f(t, x, \lambda)$ é contínua para $(t, x) \in D e \lambda \in V$ onde $V$ é uma vizinhança de $\lambda_{0}$ em $\mathbb{R}^{k}$ e $D$ é um conjunto aberto em $\mathbb{R}^{n+1}$. Considere 


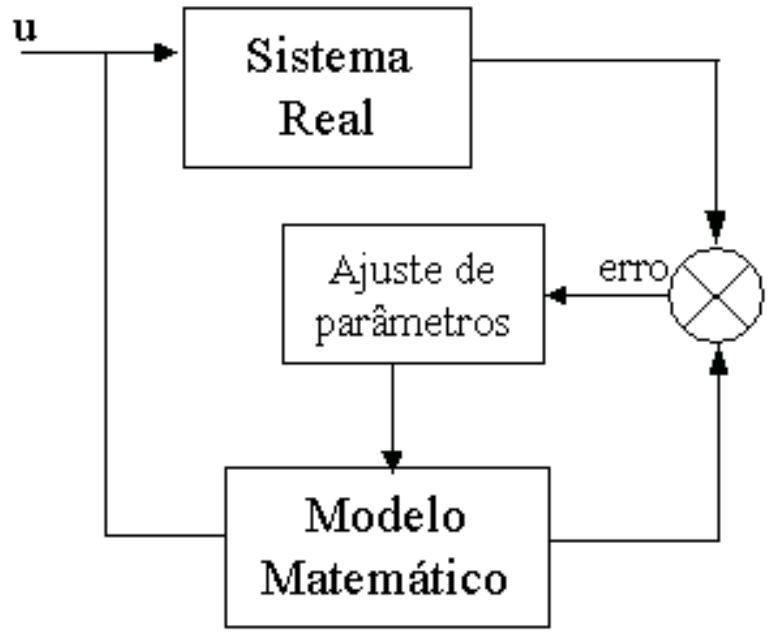

Figura 4.1: Processo de Estimação de parâmetros.

o sistema:

$$
\begin{aligned}
\dot{x} & =f\left(t, x, \lambda_{o}\right) \\
x\left(t_{o}\right) & =x_{o}
\end{aligned}
$$

onde:

- $t$ : Tempo

- $x$ : Variável de estado

- $\lambda_{o}$ : Parâmetro verdadeiro

Se o sistema 4.1 tem uma única solução não continuável $x\left(t, t_{o}, x_{o}, \lambda\right)$ definida em $\left(w_{-}, w_{+}\right)$, então para todo $(s, \eta, \lambda)$ suficientemente próximo de $\left(t_{o}, x_{o}, \lambda_{o}\right)$ o problema de valor inicial (PVI):

$$
\begin{aligned}
\dot{x} & =f(t, x, \lambda) \\
x(s) & =\eta
\end{aligned}
$$

tem uma solução $x(t, s, \eta, \lambda)$ definida em $[a, b]$ que é contínua em $\left(t, t_{o}, x_{o}, \lambda_{o}\right)$.

Do ponto de vista prático, o teorema afirma que dois sistemas de equações diferenciais (um do sistema real e o outro do modelo matemático), com condições iniciais próximas, com parâmetros também próximos terão as saídas próximas. 
Isto é muito útil, pois durante o processo de estimação de parâmetros podem existir erros na obtenção das condições iniciais das variáveis de estado no momento da amostragem (erros de medição), além disso, se tivermos um procedimento de ajuste de parâmetros, o processo de estimativa fica bem estabelecido. Considerando apenas continuidade com relação ao parâmetro $\lambda$ pode-se estabelecer o seguinte teorema:

Teorema 4.2 Dado $\epsilon>0$ arbitrariamente pequeno e $t_{1}>t_{o}, \exists \delta>0$ tal que se $\left\|\lambda-\lambda_{o}\right\|<\delta$ então $\left\|x\left(t, t_{o}, x_{o}, \lambda\right)-x\left(t, t_{o}, x_{o}, \lambda_{o}\right)\right\|<\epsilon$ para todo $t_{o} \leq t<t_{1}$.

Isto pode ser visualizado na figura (4.2). As duas soluções $x\left(t, t_{o}, x_{o}, \lambda_{o}\right)$ e $x\left(t, t_{o}, x_{o}, \lambda\right)$ ficarão próximas (dentro do tubo de raio $\epsilon$ ) se os parâmetros $\lambda_{o}$ e $\lambda$ têm valores próximos (dentro da bola de raio $\delta$ ).

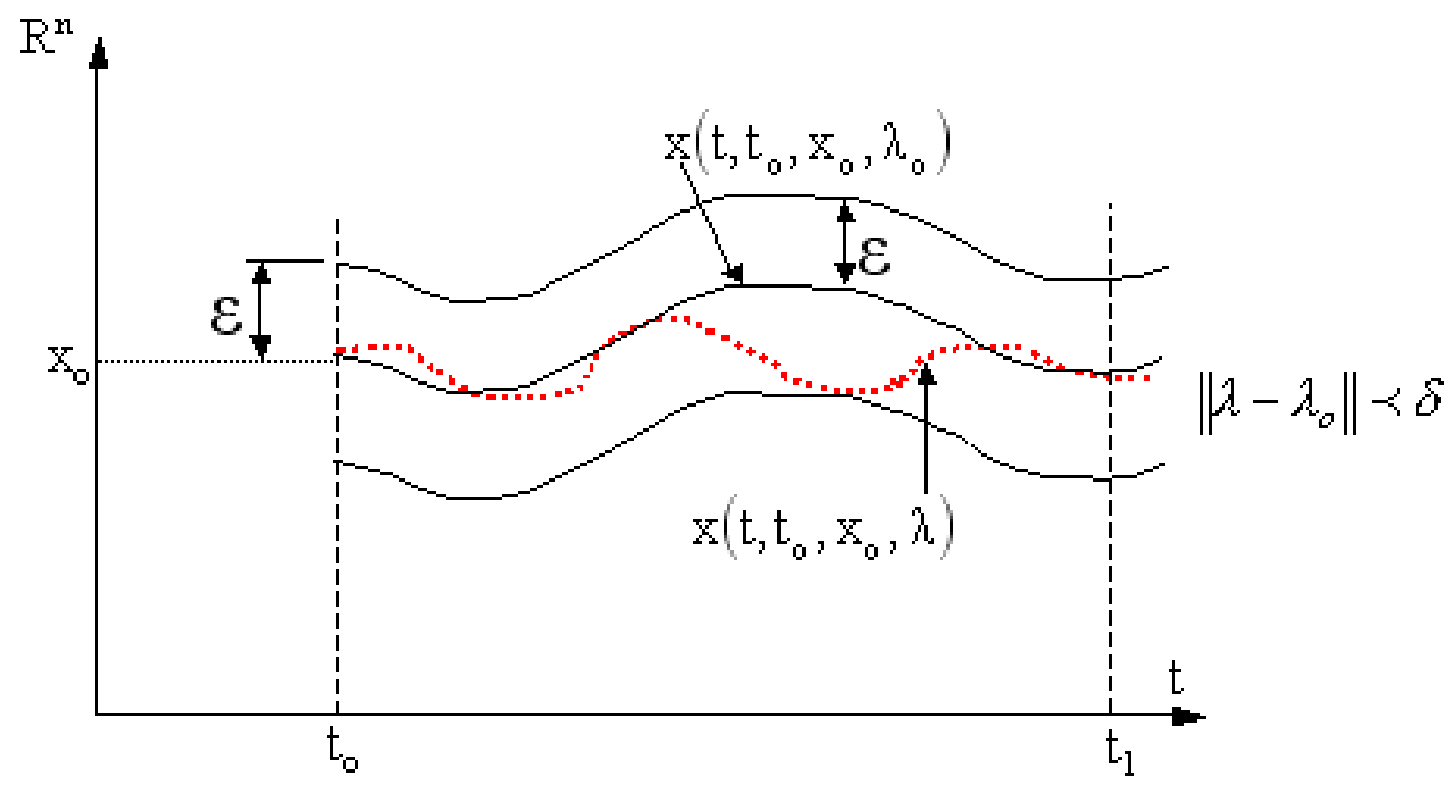

Figura 4.2: Continuidade com relação aos parâmetros.

\subsubsection{Sincronização de Sistemas}

Outro inconveniente, durante o processo de estimação, é que o modelo matemático é apenas uma representação aproximada do sistema real. Se alguma dinâmica for 
desprezada (devido as aproximações) é possível que o processo de estimação não convirja ou que os parâmetros estimados não levem a uma boa representação do sistema real.

Uma alternativa, proposta na presente dissertação, para aumentar a robustez do processo de estimação é aplicar técnicas de sincronização no procedimento de identificação de parâmetros. Na literatura existem relatos de aplicações desta técnica, mas com outros objetivos. Em (Gameiro e Rodrigues, 2000) e (Rodrigues, 1996) foram desenvolvidos métodos matemáticos para a sincronização de sistemas aplicados a codificação e decodificação de mensagens.

Em (Huang e Guo, 2004) a técnica de sincronização foi utilizada para a identificação de parâmetros de equações diferenciais de sistemas caóticos. Na referida pesquisa, os parâmetros foram estimados através de um processo de controle adaptativo utilizando funções de Lyapunov para garantir a sincronização.

A análise da sincronização considera que as trajetórias de dois sistemas de equações diferenciais, dependentes de parâmetros, ficarão próximas se estiverem acopladas de forma apropriada. Ou seja, a diferença entre duas soluções (do primeiro e do segundo sistema) torna-se pequena quando o tempo tende ao infinito. O acoplamento dos sistemas a ser considerado é do tipo unilateral, onde o segundo sistema é acoplado ao primeiro mas o primeiro é independente do segundo. Este tipo de acoplamento é conhecido como sincronização 'Master - Slave' ((Pecora e Caroll, 1990)).

Na figura 4.3 mostra-se o diagrama esquemático desta abordagem. A idéia consiste em considerar como entrada do modelo matemático algumas das saídas do sistema real.

Uma das saídas medidas do sistema real $x_{m}$ é usada como entrada do bloco modelo matemático. Este acoplamento unidirecional pode melhorar a robustez do procedimento de estimação dos parâmetros, pois faz que as saídas possam aproximar-se mesmo com estimativas inicias distantes. Com esta consideração, o processo de estimação de parâmetros pode ser considerado como um problema de sincronização de sistemas. sendo: 


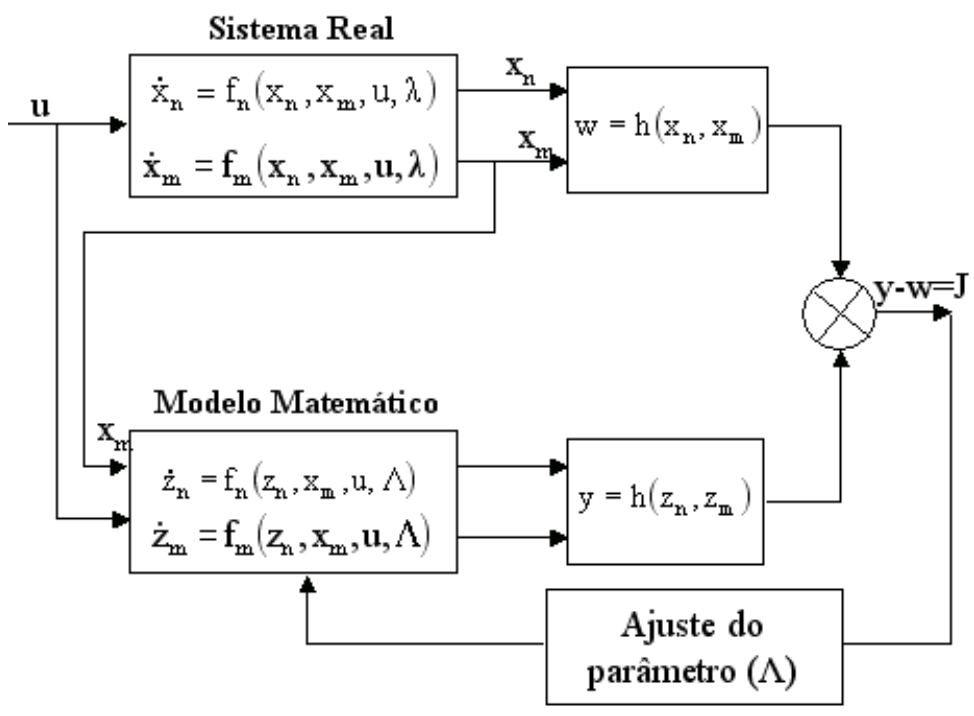

Figura 4.3: Estimação de parâmetros por sincronização.

- $\lambda$ : Parâmetro do sistema real.

- $\bigwedge$ : Parâmetro do modelo matemático.

Se ambas as saídas 'y' e 'w' sincronizarem quando os parâmetros do 'modelo Matemático' e do 'sistema real' forem próximos então o problema de identificação de parâmetros fica bem definido bastando apenas procurar por uma técnica de ajuste dos parâmetros, que no nosso caso será a técnica de mínimos quadrados juntamente com a técnica de sensibilidade de trajetória modificada pela sincronização de sistemas.

\section{Exemplo 4.1}

No exemplo a seguir, compara-se a a estimação dos parâmetros do sistema massa-mola (analisado no capítulo anterior) através da metodologia de sensibilidade de trajetória tradicional e considerando entradas de sincronização.

As equações para o 'modelo matemático' e o 'sistema real' considerando a metodologia com a técnica de sensibilidade de trajetória são mostras na figura 4.4 ,

Da mesma forma, as equações considerando a sensibilidade de trajetória com entradas de sincronização são mostradas nas figuras 4.5 .

Nas referidas figuras utiliza-se $\lambda_{f}^{p}$ por $\frac{\partial f}{\partial p}$ (f é a variável de estado e 'p' é o 
parâmetro) para facilitar a análise.

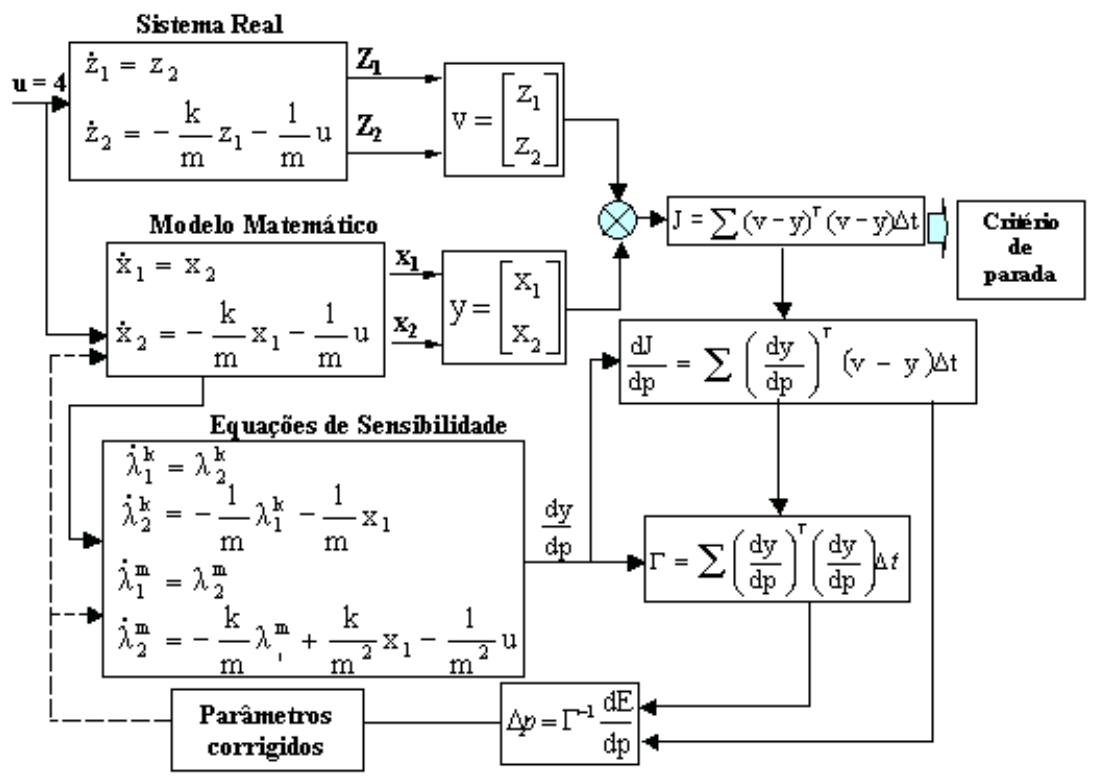

Figura 4.4: Estimação com a técnica de sensibilidade de trajetória.

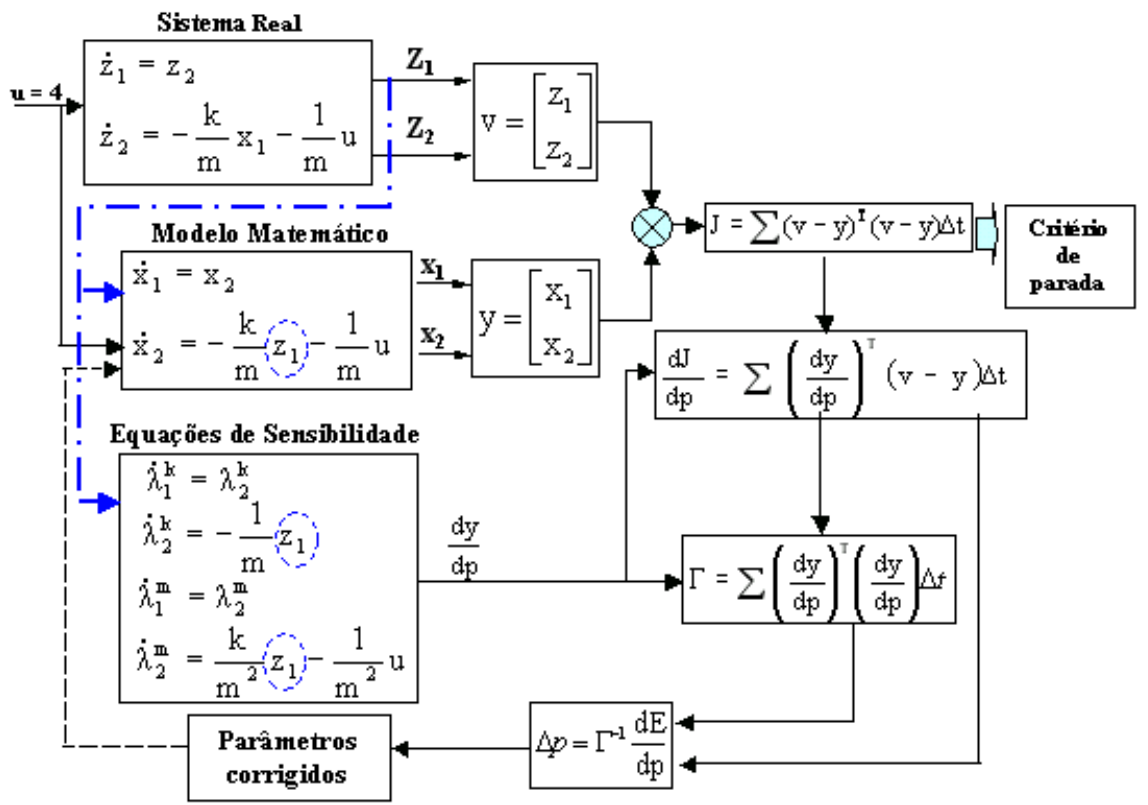

Figura 4.5: Estimação com a técnica de sensibilidade de trajetória mais sincronização.

Como pode visualizar-se na figura 4.5, aplicando as entradas de sincronização as equações do 'modelo matemático' e do 'modelo de sensibilidade' mudam em relação as equações que considerem apenas a técnica de sensibilidade de trajetória tradicional mostrado na figura 4.4 . 
Os parâmetros do sistema real são: $k_{r}=8$ e $m_{r}=2$ (verdadeiros) e os parâmetros do 'modelo matemático' são: $k_{m}=4$ e $m_{m}=3$. Nas tabelas $4.1 \mathrm{e}$ 4.2 mostram-se os resultados com as duas abordagens em cada iteração (Iter).

Tabela 4.1: Resultados com a técnica de sensibilidade de trajetória tradicional (diverge).

\begin{tabular}{|c|c|c|c|c|c|c|}
\hline Iter. & $\begin{array}{c}\text { Valor } \\
\text { Singular 1(v1) }\end{array}$ & $\begin{array}{c}\text { Valor } \\
\text { Singular 2(v1) }\end{array}$ & $\frac{v 1}{v 2}$ & $\mathrm{k}$ & $\mathrm{m}$ & $\begin{array}{c}\text { Erro(\%) } \\
(\mathrm{J})\end{array}$ \\
\hline 0 (inicial) & - & - & - & 4 & 3 & - \\
\hline 1 & 2.7485 & 0.4058 & 6.7492 & 7.3528 & 5.4597 & 5.9768 \\
\hline 2 & 0.2450 & 0.0359 & 6.8261 & 12.513 & 9.0511 & 2.7541 \\
\hline 3 & 0.0309 & 0.0044 & 7.0478 & 18.6128 & 12.3595 & 2.0498 \\
\hline 4 & 0.0077 & 0.0010 & 8.0244 & 22.0843 & 9.5180 & 1.9053 \\
\hline 5 & 0.0161 & 0.0006 & 25 & 5.5408 & -7.8172 & 1.5463 \\
\hline 6 & 35.327 & 0.1226 & 288.16 & diverge & diverge & 189.88 \\
\hline
\end{tabular}

Tabela 4.2: Resultados com a técnica de sensibilidade de trajetória tradicional mais sincronização de sistemas (converge).

\begin{tabular}{|c|c|c|c|c|c|c|}
\hline Iter. & $\begin{array}{c}\text { Valor } \\
\text { Singular 1(v1) }\end{array}$ & $\begin{array}{c}\text { Valor } \\
\text { Singular 2(v1) }\end{array}$ & $\frac{v 1}{v 2}$ & $\mathrm{k}$ & $\mathrm{m}$ & $\begin{array}{c}\operatorname{Erro}(\%) \\
(\mathrm{J})\end{array}$ \\
\hline 0 (inicial) & - & - & - & 4 & 3 & - \\
\hline 1 & 11.8179 & 0.0559 & 211.26 & 10.00 & 1.4915 & 29.16 \\
\hline 2 & 38.3089 & 1.1429 & 33.51 & 8.47 & 1.8601 & 30.42 \\
\hline 3 & 10.1812 & 1.1429 & 8.9085 & 8.011 & 1.979 & 1.1488 \\
\hline 4 & 9.0513 & 0.8863 & 10.212 & 7.9823 & 1.9885 & 0.0039 \\
\hline 5 & 9.0029 & 0.8659 & 10.3975 & 7.9824 & 1.9886 & 0.0003 \\
\hline 6 & 9.0016 & 0.8656 & 10.3989 & 7.9824 & 1.9886 & 0.0003 \\
\hline
\end{tabular}

A metodologia de sensibilidade de trajetória tradicional não conseguiu estimar os parâmetros, pois a partir da quinta iteração o parâmetro 'm' torna-se negativo e os parâmetros não convergem (tabela 4.1). 
A metodologia de sensibilidade de trajetória com a técnica de sincronização de sistemas estimou satisfatoriamente os parâmetros. Os parâmetros convergiram em 6 iterações (tabela 4.2).

No capítulo 6 , a técnica de sensibilidade de trajetória com a sincronização de sistemas será aplicado ao modelo da máquina síncrona. 


\section{Capítulo 5}

\section{Modelagem da Máquina Síncrona e dos Controladores Associados}

Para o processo de estimação de parâmetros um dos principais requisitos é que se conheça o modelo a identificar. Portanto é importante tomar uma representação adequada tanto do gerador síncrono como dos controladores associados (Sistema de excitação) para obter as equações diferenciais que descrevem de maneira satisfatória o comportamento dinâmico da máquina.

Este capítulo apresenta alguns modelos para a máquina síncrona comumente utilizados para análise de estabilidade, bem como de seus controladores associados.

\subsection{Modelagem da Máquina Síncrona}

As máquinas síncronas podem ser de dois tipos: máquinas de pólos lisos (rotor cilíndrico) e máquinas de pólos salientes. Nas unidades hidrelétricas, onde as máquinas operam em baixa velocidade (devido a grande inércia da máquina por características da turbina hidráulica), são utilizadas máquinas de pólos salientes. Em unidades termoelétricas, onde as máquinas operam em alta velocidade, são utilizadas máquinas síncronas de pólos lisos (para evitar as forças de atrito entre o rotor e o ar).

Uma máquina síncrona é constituída por um elemento girante chamado ro- 
tor, envolvido por um elemento fixo denominado estator. Aplicando corrente contínua à bobina do rotor, denominada enrolamento de campo, induz-se uma tensão senoidal nas bobinas presas ao estator. A freqüência desta tensão é determinada pela velocidade angular e pelo número de pólos magnéticos do rotor.

Além disso, na presença de transitórios, o rotor do gerador inclui outros caminhos nos quais a corrente pode ser induzida. Em alguns casos, estes caminhos são intencionalmente incluídos pelo projetista como, por exemplo, os enrolamentos de amortecimento dispostos na face polar. Em outros casos, estes caminhos são inerentes à máquina, como por exemplo, a corrente induzida (corrente de Foulcalt) no corpo do rotor sólido das máquinas síncronas de pólos lisos (IEEE, 2002), (Kimbark, 1995).

Os enrolamentos amortecedores melhoram as características de estabilidade (amortecendo as oscilações durante o transitório), mantêm e auxiliam a operação mais segura em condições de carregamento assimétrico.

Numa máquina de pólos salientes, geralmente o rotor é laminado por isso as correntes de Foulcault através do ferro do rotor são pequenas e não há necessidade de modelá-las. Nas máquinas de pólos lisos todo o rotor está preenchido com material ferromagnético portanto, as correntes de Foucault que fluem através do mesmo têm efeito significativo na dinâmica da máquina. Dessa forma, os efeitos destas correntes geralmente são modelados, uma vez que produzem amortecimento natural para a máquina.

Na análise da máquina síncrona, quando uma referência é fixa ao estator para a medida do ângulo, as grandezas eletromagnéticas, medidas com relação a esta referência, apresentam variações no tempo devido ao movimento do rotor.

A análise pode ser bastante simplificada utilizando uma referência girante com o rotor. Para tal análise, a corrente de armadura e as tensões são transformadas em dois conjuntos de variáveis ortogonais, um conjunto alinhado com o eixo magnético do enrolamento de campo, conhecido como eixo direto (eixo d), e um segundo conjunto alinhado com o eixo $90^{\circ}$ elétricos atrasado em relação o eixo do enrolamento de campo. Este segundo eixo é conhecido como eixo em quadratura 


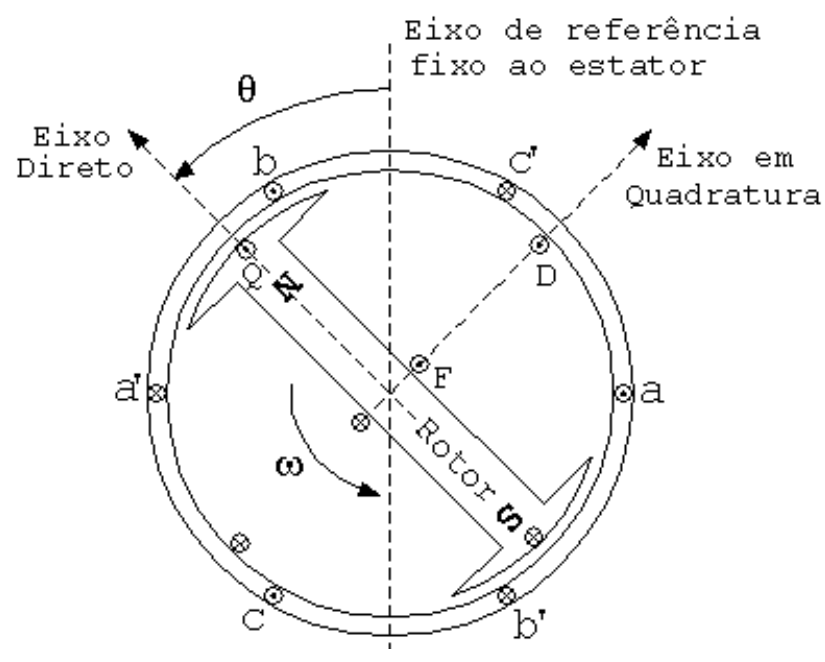

Figura 5.1: Máquina síncrona de dois pólos.

(eixo q). A transformação de variáveis mencionada anteriormente é conhecida como transformada de Park ou dqo. A transformada dqo pode ser escrita por:

$$
\left[\begin{array}{c}
i_{o} \\
i_{d} \\
i_{q}
\end{array}\right]=P\left[\begin{array}{c}
i_{a} \\
i_{b} \\
i_{c}
\end{array}\right]
$$

A matriz $\mathrm{P}$ é definida por:

$$
P=\frac{2}{3}\left[\begin{array}{ccc}
\frac{1}{\sqrt{2}} & \frac{1}{\sqrt{2}} & \frac{1}{\sqrt{2}} \\
\cos (\Theta) & \cos \left(\Theta-\frac{2 \Pi}{3}\right) & \cos \left(\Theta+\frac{2 \Pi}{3}\right) \\
\sin (\Theta) & \sin \left(\Theta-\frac{2 \Pi}{3}\right) & \sin \left(\Theta+\frac{2 \Pi}{3}\right)
\end{array}\right]
$$

\subsubsection{Equações Elétricas da Máquina Síncrona}

A máquina síncrona da figura 5.1 pode ser representada pelo circuito equivalente da figura 5.2

Para este circuito, podemos escrever a equação matricial

$$
\left[\begin{array}{c}
\mathbf{v}_{a b c} \\
\mathbf{v}_{F D Q}
\end{array}\right]=-\left[\begin{array}{cc}
\mathbf{R}_{a b c} & \mathbf{0} \\
\mathbf{0} & \mathbf{R}_{F D Q}
\end{array}\right]\left[\begin{array}{c}
\mathbf{i}_{a b c} \\
\mathbf{i}_{F D Q}
\end{array}\right]-\left[\begin{array}{c}
\dot{\lambda}_{a b c} \\
\dot{\lambda}_{F D Q}
\end{array}\right]+\left[\begin{array}{c}
\mathbf{v}_{n} \\
\mathbf{0}
\end{array}\right]
$$

sendo $\mathbf{v}_{a b c}, \mathbf{i}_{a b c}$ e $\lambda_{a b c}$ vetores com as tensões, correntes e fluxos concatenados nas fases $a, b$ e $c$, respectivamente, $\mathbf{v}_{F D Q}, \mathbf{i}_{F D Q}$ e $\lambda_{F D Q}$ vetores com as tensões, 


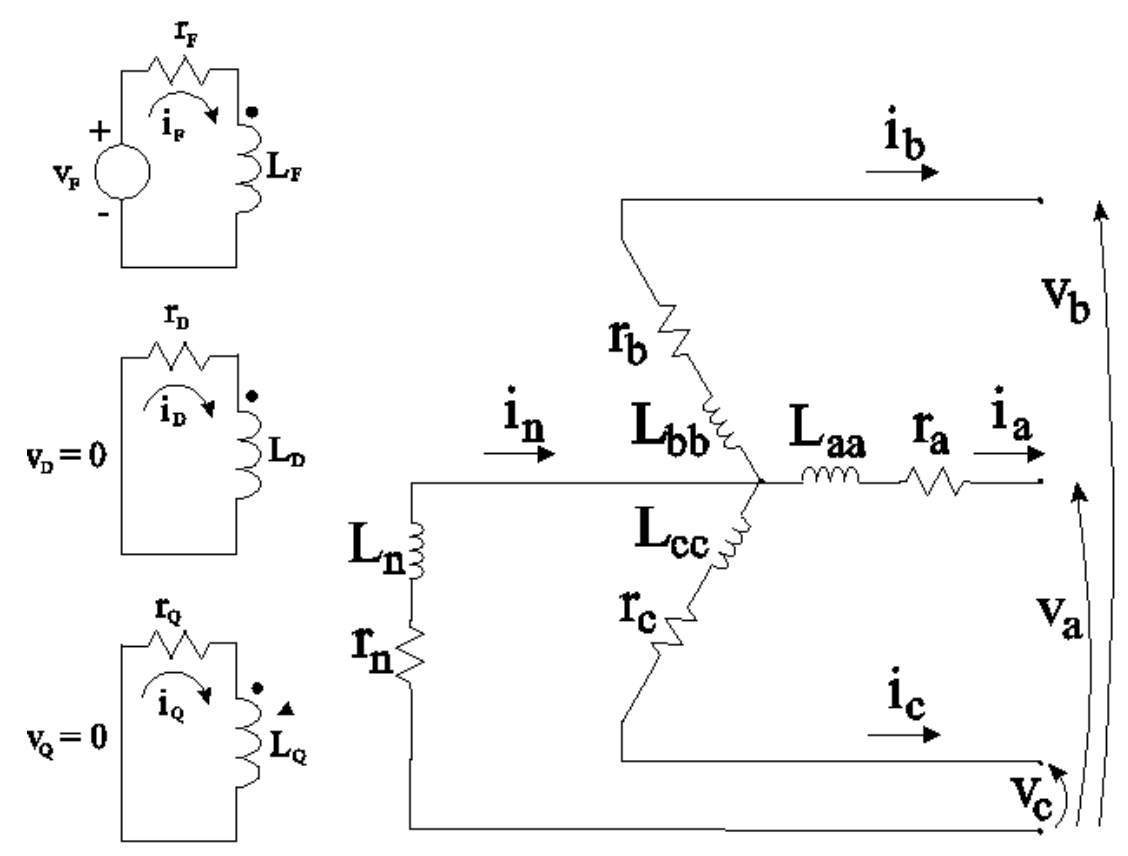

Figura 5.2: Equivalente da máquina síncrona na referência abc.

correntes e fluxos concatenados nos circuitos $F, D$ e $Q$, respectivamente, $\mathbf{R}_{a b c}$ e $\mathbf{R}_{F D Q}$ matrizes diagonais com as resistências dos circuitos $a, b, c, F, D$ e $Q$, respectivamente, e $\mathbf{v}_{n}$ um vetor com a tensão de neutro. Aplicando a transformação de Park na equação (5.3), podemos simplificá-la, obtendo (Anderson e Fouad, 1993):

$$
\begin{aligned}
{\left[\begin{array}{c}
\mathbf{v}_{0 d q} \\
\mathbf{v}_{F D Q}
\end{array}\right]=} & -\left[\begin{array}{cc}
\mathbf{R}_{0 d q} & \mathbf{0} \\
\mathbf{0} & \mathbf{R}_{F D Q}
\end{array}\right] \cdot\left[\begin{array}{c}
\mathbf{i}_{0 d q} \\
\mathbf{i}_{F D Q}
\end{array}\right] \\
& -\left[\begin{array}{c}
\dot{\lambda}_{0 d q} \\
\dot{\lambda}_{F D Q}
\end{array}\right]-\left[\begin{array}{c}
\dot{\mathbf{P}} \mathbf{P}^{-1} \lambda_{0 d q} \\
\mathbf{0}
\end{array}\right]+\left[\begin{array}{c}
\mathbf{n}_{0 d q} \\
\mathbf{0}
\end{array}\right]
\end{aligned}
$$

Sendo $\mathbf{R}_{0 d q}$ uma matriz diagonal com as resistências equivalentes e $\mathbf{v}_{0 d q}, \mathbf{i}_{0 d q}$ e $\lambda_{0 d q}$ vetores com as tensões, correntes e fluxos concatenados nos enrolamentos fictícios $0, d$ e $q$, respectivamente.

No novo sistema de coordenadas obtido após a transformação de Park, as indutâncias próprias e mútuas dos enrolamentos fictícios $0, d$ e $q$ não dependem do ângulo $\theta$. A relação entre os fluxos magnéticos e as correntes nos respectivos 
circuitos pode ser escrita da seguinte forma:

$$
\left[\begin{array}{c}
\lambda_{0} \\
\lambda_{d} \\
\lambda_{q} \\
\lambda_{F} \\
\lambda_{D} \\
\lambda_{Q}
\end{array}\right]=\left[\begin{array}{cccccc}
L_{0} & 0 & 0 & 0 & 0 & 0 \\
0 & L_{d} & 0 & k M_{F} & k M_{D} & 0 \\
0 & 0 & L_{q} & 0 & 0 & k M_{Q} \\
0 & k M_{F} & 0 & L_{F} & M_{R} & 0 \\
0 & k M_{D} & 0 & M_{R} & L_{D} & 0 \\
0 & 0 & k M_{Q} & 0 & 0 & L_{Q}
\end{array}\right]\left[\begin{array}{c}
i_{0} \\
i_{d} \\
i_{q} \\
i_{F} \\
i_{D} \\
i_{Q}
\end{array}\right]
$$

Sendo $L_{i}$ a indutância própria do enrolamento $i, M_{F}$ a indutância mútua entre os enrolamentos $F$ e $d, M_{D}$ a indutância mútua entre os enrolamentos $D$ e $d, M_{Q}$ a indutância mútua entre os enrolamentos $Q$ e $q, \omega$ a velocidade angular absoluta (em relação à referência fixa) do rotor e $k$ uma constante igual a $\sqrt{3 / 2}$. Como as indutâncias são agora constantes, podemos escrever os termos $\dot{\lambda}$ em função das correntes, a partir de (5.5). Fazendo isso, e rearranjando as equações, obtêm-se um bloco para as equações de eixo direto e outro para as de eixo em quadratura. De acordo com (Anderson e Fouad, 1993), pode-se admitir que as resistências dos circuitos do estator são iguais, e dessa forma obtemos:

$$
\begin{aligned}
& {\left[\begin{array}{c}
v_{0} \\
v_{d} \\
-v_{F} \\
v_{D}=0 \\
v_{q} \\
v_{Q}=0
\end{array}\right]=-\left[\begin{array}{cccccc}
r+3 r_{n} & 0 & 0 & 0 & 0 & 0 \\
0 & r & 0 & 0 & \omega L_{q} & \omega k M_{q} \\
0 & 0 & r_{F} & 0 & 0 & 0 \\
0 & 0 & 0 & r_{D} & 0 & 0 \\
0 & -\omega L_{d} & -\omega k M_{F} & -\omega k M_{D} & r & 0 \\
0 & 0 & 0 & 0 & 0 & r_{Q}
\end{array}\right]\left[\begin{array}{c}
i_{0} \\
i_{d} \\
i_{F} \\
i_{D} \\
i_{q} \\
i_{Q}
\end{array}\right] } \\
&--\left[\begin{array}{cccccc}
L_{0}+3 L_{n} & 0 & 0 & 0 & 0 & 0 \\
0 & L_{d} & k M_{F} & k M_{D} & 0 & 0 \\
0 & k M_{F} & L_{F} & M_{R} & 0 & 0 \\
0 & k M_{D} & M_{R} & L_{D} & 0 & 0 \\
0 & 0 & 0 & 0 & L_{q} & k M_{Q} \\
0 & 0 & 0 & 0 & k M_{Q} & L_{Q}
\end{array}\right]\left[\begin{array}{c}
i_{0} \\
i_{d} \\
i_{F} \\
i_{D} \\
i_{q} \\
\dot{i}_{Q}
\end{array}\right]
\end{aligned}
$$

sendo $v_{i}, i_{i}$ e $r_{i}$ a tensão, a corrente e a resistência do i-ésimo enrolamento e $r_{n}$ e $L_{n}$ as respectivas resistência e indutância do neutro. 
Isolando-se o vetor composto pelos termos $\dot{i}$, pode-se escrever o conjunto de equações que compõem (5.6) na forma de espaço de estados. Acrescentando-se duas equações mecânicas a este conjunto (as quais serão definidas na seção 5.1.2, obtém-se um modelo em espaço de estados para a máquina síncrona.

Em condições balanceadas, a equação matricial (5.6) pode ser escrita sem a equação de seqüência zero (pois esta equação é desacoplada das demais e pode ser resolvida separadamente no caso de condições não balanceadas).

O conjunto de equações 5.6 pode ser representado por um circuito equivalente, mostrado na figura 5.3. Devido ao novo sistema de coordenadas criado pela Transformação de Park, obtém-se uma nova representação na qual os circuitos do estator $(d$ e $q)$ são desacoplados.

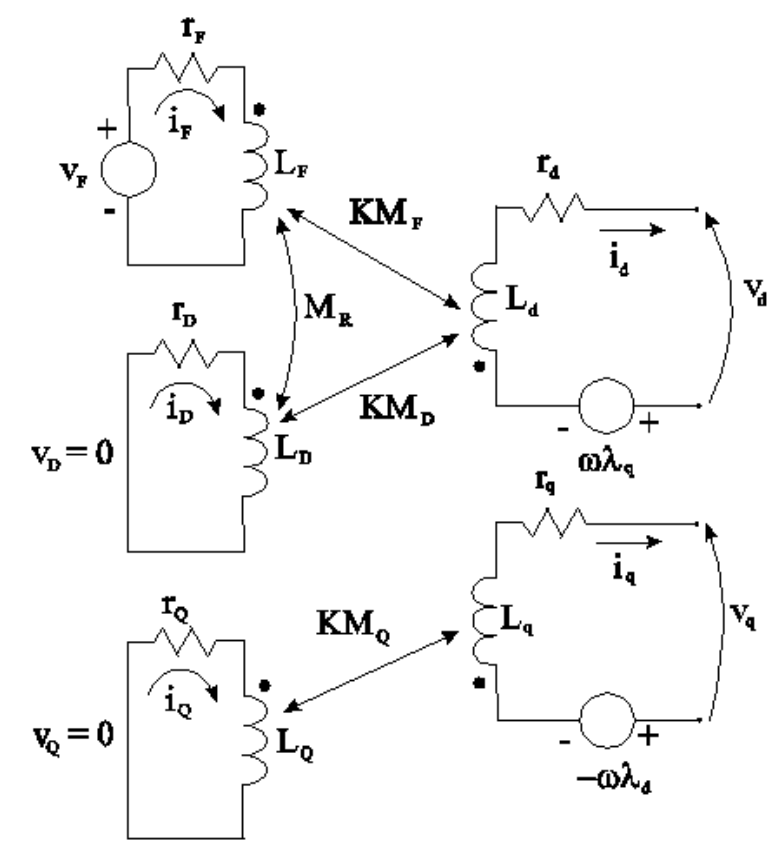

Figura 5.3: Circuito Equivalente da máquina Síncrona (referência 0dq).

A referência (Anderson e Fouad, 1993) adota uma base especial para a conversão das grandezas ao sistema p.u., de modo que o fluxo mútuo concatenado em qualquer um dos enrolamentos seja igual a todos os demais. Por essa razão, a indutância mútua entre dois enrolamentos quaisquer pode ser representada por um circuito $\mathrm{T}$, o que permite que o conjunto de equações 5.6 possa ser representado por apenas dois circuitos equivalentes, mostrados nas figuras 5.4 e 5.5 
(nas quais, em p.u., $L_{A D}=k M_{F}=k M_{D}=M_{R}$ e $L_{A Q}=k M_{Q}$ ).

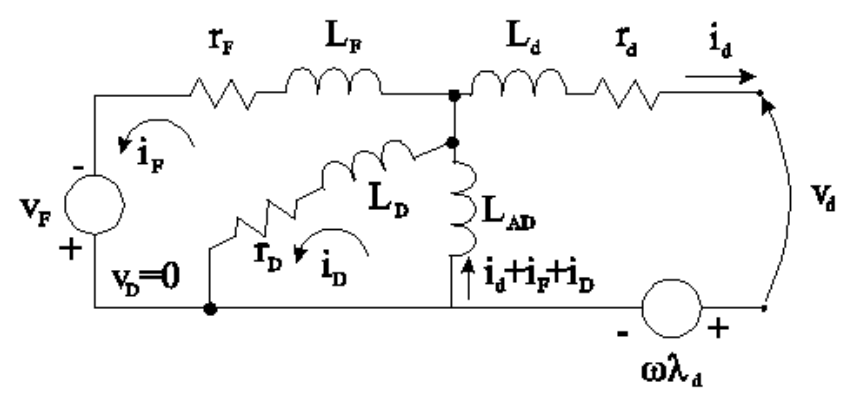

Figura 5.4: Circuito equivalente do eixo d.

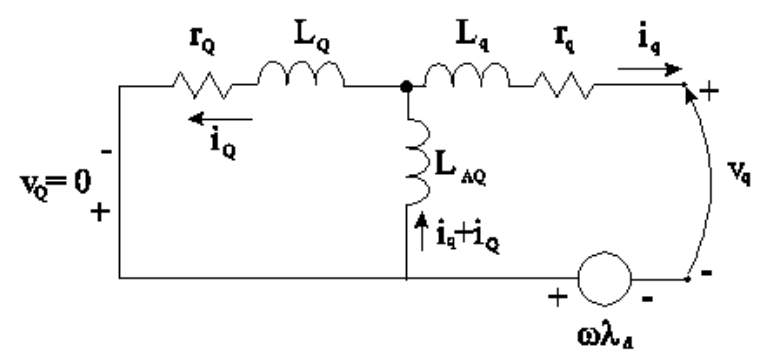

Figura 5.5: Circuito equivalente do eixo q.

O IEEE padronizou diferentes circuitos equivalentes para o gerador síncrono. Estes circuitos equivalentes mudam dependendo do número de enrolamentos amortecedores considerados.

Na figuras 5.6, 5.7 e 5.8 mostram-se três grupos de circuitos equivalentes classificados pelo IEEE. 


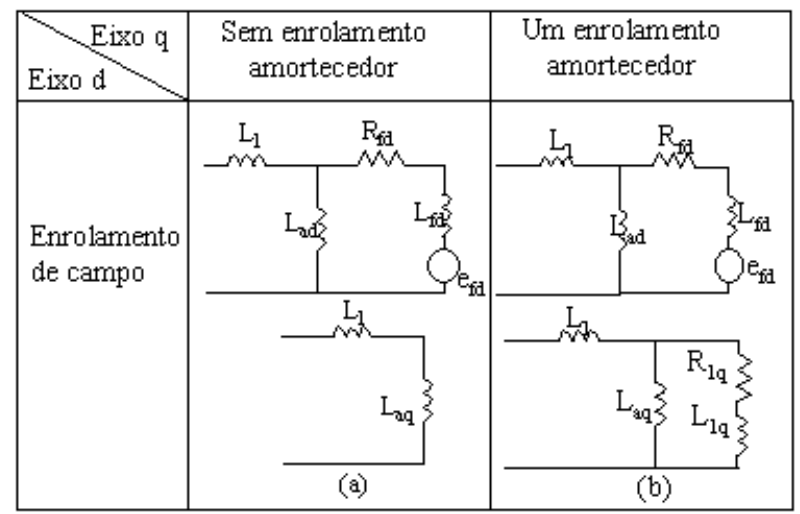

Figura 5.6: Modelo Equivalente (1) da Máquina Síncrona.

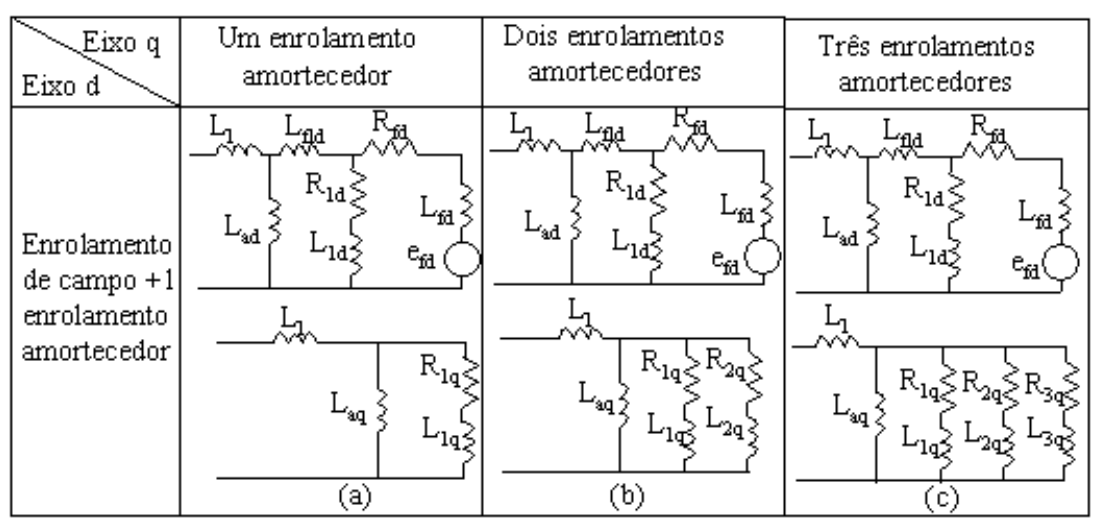

Figura 5.7: Modelo Equivalente (2) da Máquina Síncrona.

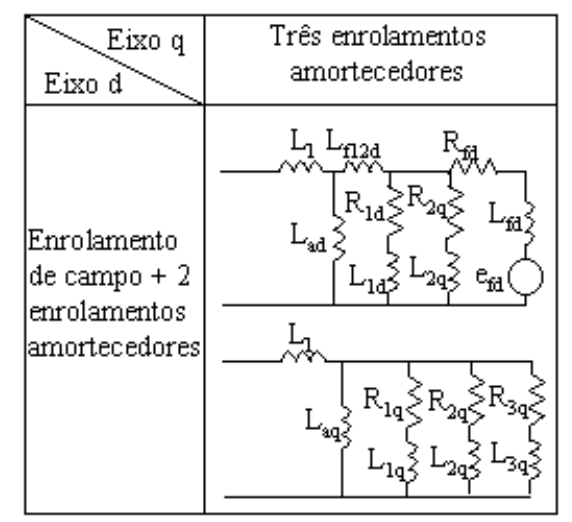

Figura 5.8: Modelo Equivalente (3) da Máquina Síncrona. 


\subsubsection{Equações Mecânicas}

As equações diferenciais que descrevem o comportamento dinâmico do sistema podem ser obtidas através de um balanço de potência em cada máquina do sistema.

Seja a máquina síncrona representada na figura 5.9 . Pelas leis físicas, sabese que o momento de inércia "J"multiplicado pela aceleração angular é igual ao torque aplicado ao rotor da máquina síncrona, isto é:

$$
J \ddot{\theta_{m}}=T_{a}
$$

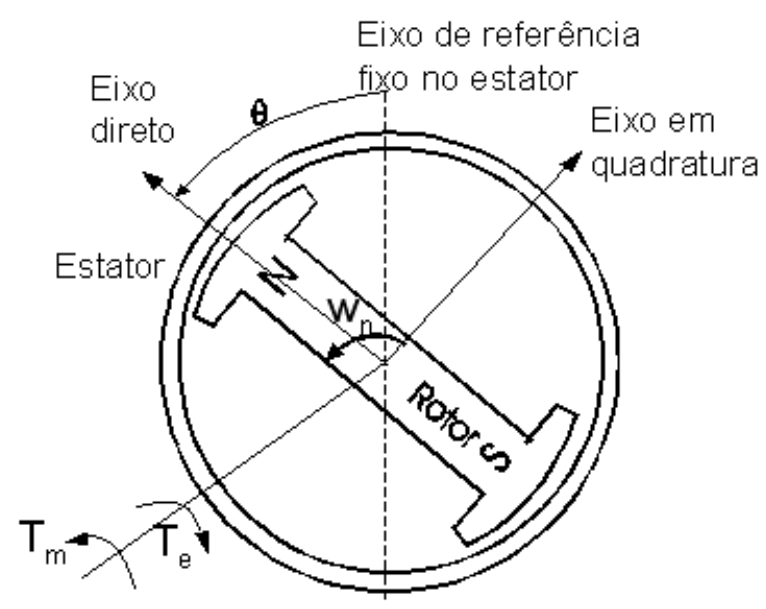

Figura 5.9: Máquina Síncrona de dois pólos.

Na equação anterior, J é o momento de inércia do rotor, $T_{a}$ é o torque de aceleração do rotor e $\theta_{m}$ é o ângulo mecânico do eixo direto do rotor com relação a um eixo de referência estacionário.

O torque $T_{a}$ resulta da diferença entre os torques mecânico e elétrico aplicados ao rotor e é dado pela seguinte expressão:

$$
T_{a}=T_{m}-T_{e}
$$

Como o ângulo $\theta_{m}$ é medido em relação a uma referência estacionária, este será uma função senoidal do tempo em condições de regime permanente. Isso 
é um grande inconveniente na análise do sistema de potência na presença de transitórios. Para evitar este inconveniente podemos utilizar um outro sistema de referência, girando agora à velocidade síncrona. O ângulo do rotor, medido em relação a esta nova referência, será chamado de $\delta_{m}$ e será dado por:

$$
\delta_{m}(t)=\theta_{m}(t)-\left(w_{o m} t+\pi / 2+\alpha\right)
$$

Na equação acima, $w_{o m}$ é a velocidade síncrona e $\left(w_{o m} t+\Pi / 2+\alpha\right)$ define a referência girante. Se tomarmos $t=0$ como sendo o instante em que o eixo em quadratura está alinhado com o eixo de referência fixo ao estator, $\alpha$ será o ângulo de defasagem entre a referência fixa e a referência girante em $t=0$. Por sua vez, o ângulo do rotor $\delta_{m}(t)$, com a hipótese acima, será o ângulo entre o eixo em quadratura e a referência girante.

Apesar do novo sistema de referência estar em movimento, ele continua sendo um sistema de referência inercial. Com isso, as equações de aceleração não se alteram ao passarmos para este novo sistema de referência. Isso pode ser verificado facilmente, pois:

$$
\begin{array}{r}
\dot{\Theta}_{m}=w_{s}+\dot{\delta}_{o m} \\
\ddot{\delta}_{m}=\ddot{\theta}_{m}
\end{array}
$$

A equação ( 5.7) na nova referência pode ser escrita como:

$$
J \ddot{\delta}_{m}=T_{m}-T_{e}
$$

Para obter um conjunto de equações diferenciais que defina um espaço de estados para a máquina, é mais conveniente escrever a equação ( 5.12 em termos do ângulo elétrico $\delta_{e}$, que é o ângulo formado entre a referência girante e o eixo do campo magnético que envolve o rotor, pois o torque elétrico $T_{e}$ será uma função deste ângulo. O ângulo do rotor $\delta_{m}$ e o ângulo elétrico $\delta_{e}$ estão relacionados por:

$$
\delta_{e}=\frac{p}{2} \delta_{m}
$$


sendo p o número de pólos do rotor. Define-se a velocidade do campo girante da mesma forma:

$$
w_{e}=\dot{\delta}_{e}=\frac{p}{2} \dot{\delta}_{m}
$$

Nessas novas variáveis, a equação (5.12) pode ser escrita como:

$$
\frac{2 J}{p} \ddot{\delta}_{e}=\frac{2 J}{p} \dot{w}_{e}=T_{m}-T_{e}
$$

O momento de inércia $\mathrm{J}$ da máquina é um parâmetro que não é geralmente fornecido pelos fabricantes. Usualmente, os fabricantes fornecem um outro parâmetro, chamado de constante de inércia H. Esta constante é definida como a energia armazenada no rotor $W_{k}$, quando este gira à velocidade síncrona, dividida pela potência base trifásica da máquina $S_{B}$, ou seja,

$$
H=\frac{W_{k}}{S_{B}}=\frac{J w_{o m}^{2}}{2 S_{B}}
$$

sendo $w_{o m}$ a velocidade mecânica síncrona do sistema. Notando que $S_{B} / w_{o m}$ define um torque base $T_{B}$ e definindo a velocidade síncrona do campo girante através de

$$
w_{o e}=\frac{p}{2} w_{o m}
$$

podemos escrever a equação (5.15) como:

$$
\frac{2 H}{w_{o e}} \dot{w}_{e}=\frac{T_{m}}{T_{B}}-\frac{T_{e}}{T_{B}}
$$

Definindo agora $w_{u}=w_{e} / w_{o e}, T_{m u}=T_{m} / T_{B}$ e $T_{e u}=T_{B}$ como sendo os valores em p.u. da velocidade angular do campo(em relação à referência girante), torque mecânico e torque elétrico, respectivamente, obtemos:

$$
2 H \dot{w}_{u}=T_{m u}-T_{e u}
$$


Esta equação é chamada de equação de oscilação ou equação de "swing". Baseados na consideração de que a velocidade angular $w_{m}$ tem uma variação muito pequena durante o período transitório, pode-se considerar que o momento angular do rotor $M_{m}=J w_{m}$ é constante. Multiplicando ambos lados da equação ( 5.15) por $w_{m}$, podemos obter uma nova equação de "swing" que tem como parâmetro o momento angular $M_{m}$, constante por hipótese. Obviamente, um erro decorrente desta hipótese estará presente (Anderson e Fouad, 1993), entretanto um termo de amortecimento $D w_{m}$ pode ser incluído na nova equação para compensar este erro, resultando em :

$$
M_{m} \dot{w}_{m}+D w_{m}=P_{m}-P e
$$

As constantes $\mathrm{M}$ e $\mathrm{H}$ podem relacionam-se através de: $M=H /\left(\pi f_{o}\right)$. Substituindo o valor de M na equação (5.20) e expressando-o em valores p.u. obtém-se:

$$
2 H \dot{w}_{u}+D w_{u}=\frac{P_{m}}{w_{m u}}-\frac{P e}{w_{m u}}
$$

sendo $P_{m u}$ e $P_{e u}$ os valores em p.u. das potências mecânica e elétrica, respectivamente, e $w_{m u}$ o valor em p.u. da velocidade angular. Esta equação, juntamente com a definição de velocidade angular do campo girante (em relação à referência girante), equação (5.22), fornecem uma representação em espaço de estados para a máquina síncrona.

$$
\dot{\delta}=w_{e}
$$

\subsubsection{Modelos Simplificados}

Para facilitar a estimativa dos parâmetros da máquina síncrona, algumas simplificações nos modelos são necessárias.

O modelo de dois eixos, para a máquina de pólos lisos, e o modelo de um eixo, para a máquina de pólos salientes, são apresentados. O modelo clássico é também comentado. 
É necessário adotar um sistema de normalização adequado para as modelagens de um e dois eixos e para a modelagem clássica da rede. Estes modelos possuem apenas grandezas referidas ao estator. Sendo assim, não há necessidade de representação das indutâncias mútuas entre estator e rotor como circuitos $\mathrm{T}$. Desta forma os valores base do rotor não tem influência nestas modelagens. As grandezas de base que serão adotadas neste texto são:

- $S_{B}$ : Potência base trifásica do estator;

- $V_{B}$ : Tensão de linha do estator.

Escolhidas estas duas grandezas (além da base de tempo, que pode ser tomada como $t_{B}=1 \mathrm{~s}$ ), todas as grandezas ficam automaticamente determinadas. Uma análise mais profunda da escolha dos valores base estão dadas em (Rodrigo et al., 2000), (Anderson e Fouad, 1993).

\subsubsection{Modelo de dois eixos}

O modelo de 2 eixos refere-se a uma máquina de rotor cilíndrico. Este modelo é adequado para uma máquina sem enrolamentos amortecedores. Isto permite que se faça uma primeira simplificação no modelo de sétima ordem eliminando em (5.6) as equações de $v_{D}$ e $v_{Q}$. Esta modelagem despreza os termos $\dot{\lambda}$ e considera $w_{m}=1$ p.u. nas equações do estator.

Utilizando as equações (5.5) e (5.6) e as considerações feitas em (Anderson e Fouad, 1993) obtém-se as equações do modelo de dois eixos:

$$
\begin{aligned}
& \dot{E}_{q}^{\prime}=\frac{1}{T_{d o}^{\prime}}\left[E_{f d}-E_{q}^{\prime}+\left(x_{d}-x_{d}^{\prime}\right) I_{d}\right] \\
& \dot{E}_{d}^{\prime}=\frac{-1}{T_{q o}^{\prime}}\left[E_{d}^{\prime}+\left(x_{q}-x_{q}^{\prime}\right) I_{q}\right]
\end{aligned}
$$

As equações (5.23) e (5.24), juntamente com a equação de velocidade

$$
\dot{w}_{e}=\frac{1}{2 H}\left[P_{m}-P_{e}\right]
$$


e a equação de ângulo

$$
\dot{\delta}=w_{e}
$$

formam o conjunto de equações diferenciais que define o espaço de estados para o modelo de dois eixos. As equações algébricas

$$
\begin{aligned}
& V_{q}=E_{q}^{\prime}-r I_{q}+x_{d}^{\prime} I_{d} \\
& V_{d}=E_{d}^{\prime}-r I_{d}-x_{q}^{\prime} I_{q}
\end{aligned}
$$

completam o equacionamento do modelo de dois eixos.

\subsubsection{Modelo de um eixo}

O modelo de um eixo utiliza ou adota as mesmas hipóteses do modelo de dois eixos no equacionamento de uma máquina de pólos salientes. A diferença básica, neste caso, é a inexistência do circuito de eixo em quadratura no rotor, logo $E_{d}^{\prime}$ será sempre igual a zero. Desta forma, podem-se obter as equações para o modelo de um eixo simplesmente eliminando a equação diferencial de $E_{d}^{\prime}$ no modelo anterior de dois eixos.

\subsubsection{Modelo Clássico}

Neste modelo, admite-se que o fluxo no enrolamento de campo é constante. Desta forma, a tensão $E_{q}^{\prime}$, proporcional a este fluxo, pode também ser considerada constante. Sendo assim, todas as equações elétricas da máquina tornam-se equações algébricas, restando equações de velocidade (5.25) e ângulo (5.26) na formulação do espaço de estados.

O modelo clássico permite representar o gerador durante o transitório por uma tensão de magnitude fixa atrás de uma reatância equivalente (reatância transitória).

\subsubsection{Modelagem final da máquina síncrona}

Um resumo das equações para cada um dos modelos da máquina síncrona descritas nas subseções anteriores são dadas aqui. No caso do modelo de 'dois eixos' é 
incluído a ação do amortecimento através da constante D (constante de amortecimento).

1.- Modelo de dois eixos

$$
\begin{aligned}
\dot{\delta} & =w_{e} \\
\dot{w}_{e} & =\frac{w_{o}}{2 H}\left[P_{m}-E_{q}^{\prime} I_{q}-E_{d}^{\prime} I_{d}-\left(x_{d}-x_{q}^{\prime}\right) I_{d} I_{q}-\frac{D}{w_{o}} w_{m}\right] \\
\dot{E}_{q}^{\prime} & =\frac{1}{T_{d o}^{\prime}}\left[E_{f d}-E_{q}^{\prime}+\left(x_{d}-x_{d}^{\prime}\right) I_{d}\right] \\
\dot{E}_{d}^{\prime} & =\frac{-1}{T_{q o}^{\prime}}\left[E_{d}^{\prime}+\left(x_{q}-x_{q}^{\prime}\right) I_{q}\right] \\
V_{q} & =E_{q}^{\prime}-r I_{q}+x_{d}^{\prime} I_{d} \\
V_{d} & =E_{d}^{\prime}-r I_{d}+x_{d}^{\prime} I_{q}
\end{aligned}
$$

2.- Modelo de um eixo

$$
\begin{aligned}
\dot{\delta} & =w_{e} \\
\dot{w}_{e} & =\frac{w_{o}}{2 H}\left[P_{m}-E_{q}^{\prime} I_{q}-\left(x_{d}^{\prime}-x_{q}^{\prime}\right) I_{d} I_{q}-\frac{D}{w_{o}} w_{e}\right] \\
\dot{E}_{q}^{\prime} & =\frac{1}{T_{d o}^{\prime}}\left[E_{f d}-E_{q}^{\prime}+\left(x_{d}-x_{d}^{\prime}\right) I_{d}\right] \\
V_{q} & =\quad E_{q}^{\prime}-r I_{q}+x_{d}^{\prime} I_{d} \\
V_{d} & =-r I_{d}-x_{q}^{\prime} I_{q}
\end{aligned}
$$

Uma grande simplificação no modelo de um eixo se obtém fazendo a seguinte aproximação $x_{q}^{\prime}=x_{d}^{\prime}$. Com isto o gerador pode ser representado por uma tensão variável $E_{q}^{\prime}$ atrás de uma reatância transitória $x_{d}^{\prime}$.

As equações da rede podem ser introduzidas dentro das equações diferenciais da máquina síncrona, isto facilita a análise.

Assim, as novas equações para o modelo de um eixo são as seguintes: 


$$
\begin{aligned}
\dot{\delta}_{i}= & w_{e i} \\
\dot{w}_{e i}= & \frac{w_{o}}{2 H}\left[P_{m}-\frac{D}{w_{o}} w_{e}-\sum_{j=1}^{n} E_{q i}^{\prime} E_{q j}^{\prime}\left(G_{i j} \cos \left(\delta_{i}-\delta_{j}\right)-\right.\right. \\
& \left.\left.B_{i j} \operatorname{sen}\left(\delta_{i}-\delta_{j}\right)\right)\right] \\
\dot{E}_{q}^{\prime}= & \frac{1}{T_{d o}^{\prime}}\left[E_{f d i}-E_{q i}^{\prime}+\left(x_{d i}-x_{d i}^{\prime}\right) \sum_{j=1}^{n}\left(G_{i j} E_{q j}^{\prime} \operatorname{sen}\left(\delta_{j}-\delta_{i}\right)+\right.\right. \\
& \left.\left.B_{i j} E_{q j}^{\prime} \cos \left(\delta_{j}-\delta_{i}\right)\right)\right]
\end{aligned}
$$

3.- Modelo Clássico:

$$
\begin{aligned}
\dot{w}_{e} & =\frac{w_{o}}{2 H}\left[P_{m}-\frac{E^{\prime} \cdot V t}{x_{d}^{\prime}} \operatorname{sen}(\delta-\theta)\right] \\
\dot{\delta} & =w_{e} \\
V_{t} & =E^{\prime}-r I_{t}+x_{d}^{\prime} I_{t}
\end{aligned}
$$

sendo:

- $V_{t}$ : Tensão terminal.

- $I_{t}$ : Corrente terminal.

- $E^{\prime}$ : Tensão interna.

- $r$ : Resistência de armadura. 


\subsection{Modelagem do Sistema de Excitação}

O sistema de excitação fornece uma apropriada tensão de campo para manter a tensão dos terminais da máquina a um valor desejado. Uma importante característica de controle do sistema de excitação é a resposta rápida às variações de tensão durante condições normais e de emergência. O sistema de excitação está composto basicamente por o regulador, amplificador e excitatriz. O regulador mede a tensão atual regulada e determina o desvio de tensão. O desvio de tensão é amplificada e utilizada para mudar a corrente de campo do excitador. Isto, por sua vez, muda a saída da tensão de campo que muda o nível de excitação para o gerador.

O IEEE padronizou modelos de sistemas de excitação que são amplamente utilizados nas simulações computacionais e são apresentadas em (IEEE, 1992). Neste trabalho utilizaremos dois sistemas de excitação para identificar os seus parâmetros, a primeira classificada pela IEEE como tipo ST1A e a segunda como tipo DC1A.

\subsubsection{Sistema de excitação ST1A}

O modelo do sistema de excitação considerado é mostrado na figura (5.10). Este modelo representa um sistema de excitação tipo ST1A com regulador automático de tensão ,AVR (do inglês, Automatic Voltage Regulator).

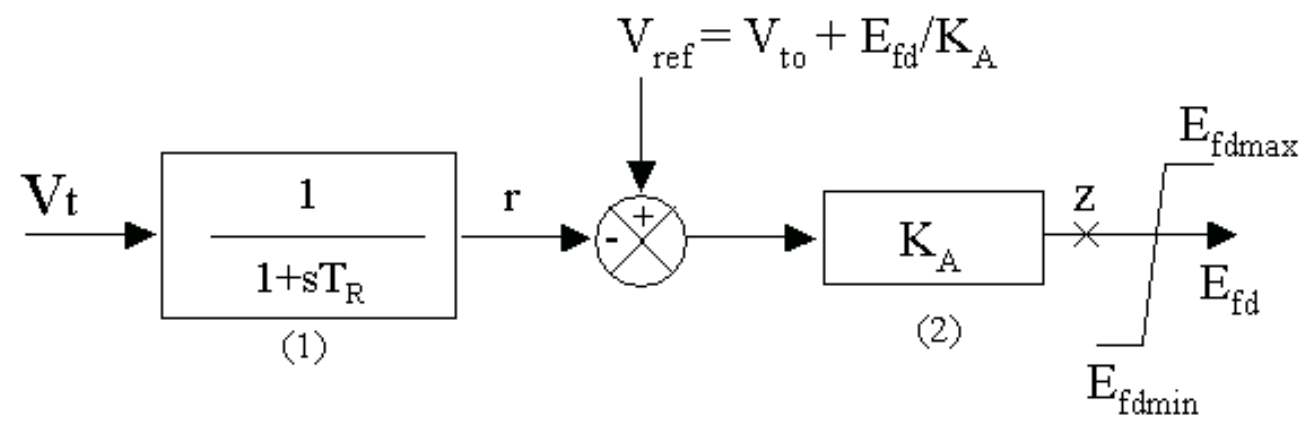

Figura 5.10: Sistema de excitação ST1A.

O desenvolvimento das equações diferenciais deste modelo são mostrados a 
seguir. Na saída do bloco (1) tem-se:

$$
\begin{aligned}
\frac{r}{V_{t}} & =\frac{1}{1+s T_{R}} \\
r+s r T_{R} & =V_{t} \\
s r & =\frac{V_{t}-R}{T_{R}}
\end{aligned}
$$

Na saída do bloco (2) tem-se:

$$
\begin{aligned}
\left(V_{r e f}-r\right) K_{A} & =E_{f d} \\
r & =-\frac{E_{f d}}{K_{A}}+V_{r e f}
\end{aligned}
$$

Substituindo a equação (5.48) na equação (5.47) tem-se:

$$
\begin{aligned}
s\left(-\frac{E_{f d}}{K_{A}}+V_{r e f}\right) & =\frac{V_{t}-\left(-\frac{E_{f d}}{K_{A}}+V_{r e f}\right)}{T_{R}} \\
-s \frac{E_{f d}}{K_{A}} & =\frac{V_{t}-\frac{-E_{f d}}{K_{A}}+V_{r e f}}{-T_{R}} \\
s E_{f d} & =-\frac{K_{A}\left(V_{t}-V_{r e f}\right)+K_{A} V_{r e f}}{-T_{R}} \\
\frac{d E_{f d}}{d t} & =\frac{1}{T_{R}}\left(K_{A}\left(V_{r e f}-V_{t}\right)-E_{f d}\right)
\end{aligned}
$$

A ação dos limites do sistema de excitação é considerada através das seguintes restrições:

- Se $E_{f d}>E_{f d \max }: E_{f d}=E_{f d \max }$

- Se $E_{f d}<E_{f d m i n}: E_{f d}=E_{f d m i n}$

\subsubsection{Sistema de excitação DC1}

O diagrama de bloco do sistema de excitação DC1 é mostrado na figura (5.11). Esta representação inclui função de transferência para o regulador, amplificador, excitador, e um bloco de realimentação. O bloco de realimentação modifica a resposta para eliminar as oscilações indesejáveis e os sobre-sinais instantâneos da tensão do regulador. Os efeitos desmagnetizantes pela saturação são incluídos por intermedio do bloco 5 . 


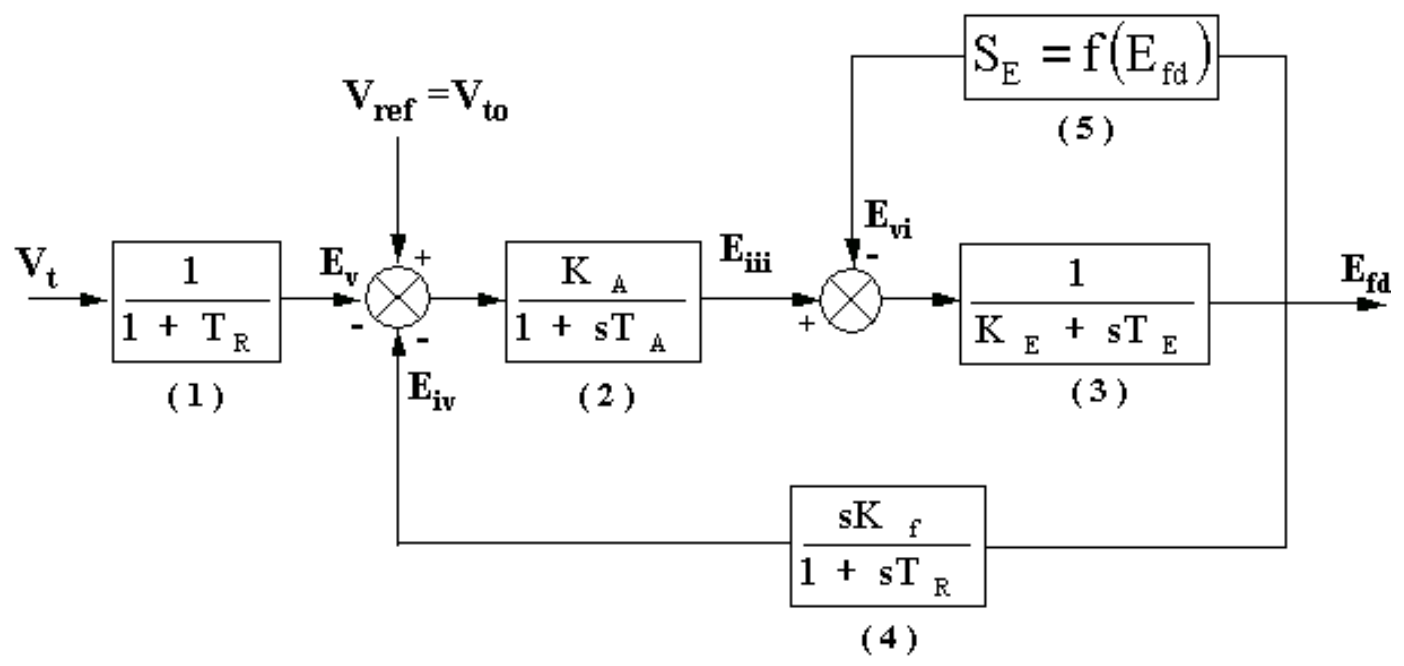

Figura 5.11: Sistema de excitação DC1

Do bloco (1) tem-se:

$$
\begin{aligned}
\frac{E_{v}}{V_{t}} & =\frac{1}{1+s T_{R}} \\
E_{v}+s E_{v} T_{R} & =V_{t} \\
\frac{d E_{v}}{d t} & =\frac{1}{T_{R}}\left(V_{t}-E_{v}\right)
\end{aligned}
$$

Do bloco (2) tem-se:

$$
\begin{aligned}
\frac{E_{i i i}}{V_{r e f}-E i v-E v} & =\frac{K_{A}}{1+s T_{A}} \\
T_{A} s E_{i i i}+E_{i i i} & =K_{A}\left(V_{r e f}-E_{i v}-E_{v}\right) \\
\frac{E_{i i i}}{d t} & =\frac{1}{T_{A}}\left(K_{A}\left(V_{r e f}-E_{i v}-E_{v}\right)-E_{i i i}\right)
\end{aligned}
$$

Do bloco (3) tem-se:

$$
\begin{aligned}
\frac{E_{f d}}{E_{i i i}-E_{i v}} & =\frac{1}{K_{E}+s T_{E}} \\
K_{E} E_{f d}+T_{E} s E_{f d} & =E_{i i} \\
\frac{E_{f d}}{d t} & =\frac{1}{T_{E}}\left(E_{i i i}-E_{v i}-K_{E} E_{f d}\right)
\end{aligned}
$$

A função de saturação pode ser aproximada por uma função exponencial, isto é:

$$
S_{E}=f\left(E_{f d}\right)=A e^{B E_{f d}}
$$


Os coeficientes A e B são calculados dos dados de saturação, onde $S_{E}$ e $E_{f d}$ são especificados em dois pontos, usualmente na tensão de 'ceiling ${ }^{1}$ e a $75 \%$ da tensão 'ceiling'.

Do bloco 5 tem-se:

$$
E_{v i}=A e^{B E_{f d}} E_{f d}
$$

Substituindo a equação (5.53) na equação (5.54) tem-se:

$$
\frac{d E_{f d}}{d t}=\frac{1}{T_{E}}\left(E_{i i i}-\left(A e^{B E_{f d}}+K_{E}\right) E_{f d}\right)
$$

Do bloco 4 tem-se:

$$
\begin{aligned}
\frac{E_{i v}}{E_{f d}} & =\frac{K_{F} s}{1+s T_{F}} \\
E_{i v}+T_{F} s E_{i v} & =K_{F} s E_{f d} \\
\frac{d E_{i v}}{d t} & =\left(K_{F} \frac{d E_{f d}}{d t}-E_{i v}\right)
\end{aligned}
$$

As equações diferenciais do sistema são então:

$$
\begin{aligned}
\frac{d E_{v}}{d t} & =\frac{1}{T_{R}}\left(V_{t}-E_{v}\right) \\
\frac{d E_{i i i}}{d t} & =\frac{1}{T_{A}}\left(K_{A}\left(V_{r e f}-E_{i v}-E_{v}\right)-E_{i i i}\right) \\
\frac{d E_{f d}}{d t} & =\frac{1}{T_{E}}\left(E_{i i i}-\left(A e^{B E_{f d}}+K_{E}\right) E_{f d}\right) \\
\frac{d E_{i v}}{d t} & =\frac{1}{T_{F}}\left(K_{F} \frac{d E_{f d}}{d t}-E_{i v}\right)
\end{aligned}
$$

Estas equações serão utilizadas no próximo capítulo para simular o sistema real. O parâmetro $K_{E}$ é calculado de tal maneira que $E_{i i i}(0)=0$ (ver figura 5.11), isto é:

$$
K_{E}=-A e^{B E_{f d}(0)}
$$

As condições iniciais para a solução das equações diferenciais mostradas anteriormente são: $E_{v}(0)=V_{t}(0), E_{i i i}(0)=0, E_{f d}(0), E_{i v}(0)=0$.

\footnotetext{
${ }^{1}$ máxima tensão do sistema de excitação
} 


\section{Capítulo 6}

\section{Aplicação da técnica de sensibilidade de trajetória para a estimação de parâmetros do} gerador síncrono e do sistema de excitação

\subsection{Introdução}

Neste capítulo apresenta-se a aplicação da técnica de sensibilidade de trajetória para a estimação dos parâmetros do gerador e do sistema de excitação. São analisadas as diferentes dificuldades durante a estimação dos parâmetros.

A metodologia é aplicada para estimar os parâmetros dos diferentes modelos da máquina síncrona (modelo clássico, modelo de um eixo, e modelo de dois eixos). É proposta a estimação por etapas, aplicada a estimação dos parâmetros do modelo clássico para depois ser usado para estimar os parâmetros do modelo de 'um eixo'.

Também são realizados diferentes testes para estimar os parâmetros do modelo de 'dois eixos' da máquina síncrona. Neste ponto é utilizada a teoria de 
sincronização de sistemas para melhorar a robustez da metodologia original. Posteriormente a metodologia é aplicada para estimar os parâmetros mecânicos da máquina síncrona.

No final a metodologia é aplicado para estimar os parâmetros de dois tipos de sistema de excitação padronizados pelo IEEE (tipo ST1A e DC1).

\subsubsection{Considerações práticas para a realização dos testes}

Os testes aqui neste trabalho são obtidos através de simulações, porém a experiência adquirida com estes testes pode ser utilizada em futuros testes em sistemas reais como mostra a figura 6.1 .

A metodologia estima os parâmetros a partir dos dados da perturbação medidos com o gerador conectado à rede. Os medidores são instalados e ficam aguardando a ocorrência de uma perturbação externa suficientemente grande para excitar as variáveis dinâmicas do sistema cujos parâmetros desejam-se estimar. Uma vez detectada a perturbação os dados referentes a um certo período de tempo são utilizados como medidas no Sistema Real. Algumas destas medidas são utilizados como variáveis de entrada no modelo matemático e outras como variáveis de saídas. As entradas no modelo matemático fornecem uma saída que é comparada com as variáveis de saída do sistema real. A diferença destas saídas é minimizada através do algoritmo de atualização de parâmetros. Isto é realizado sucessivamente até a convergência dos parâmetros. 


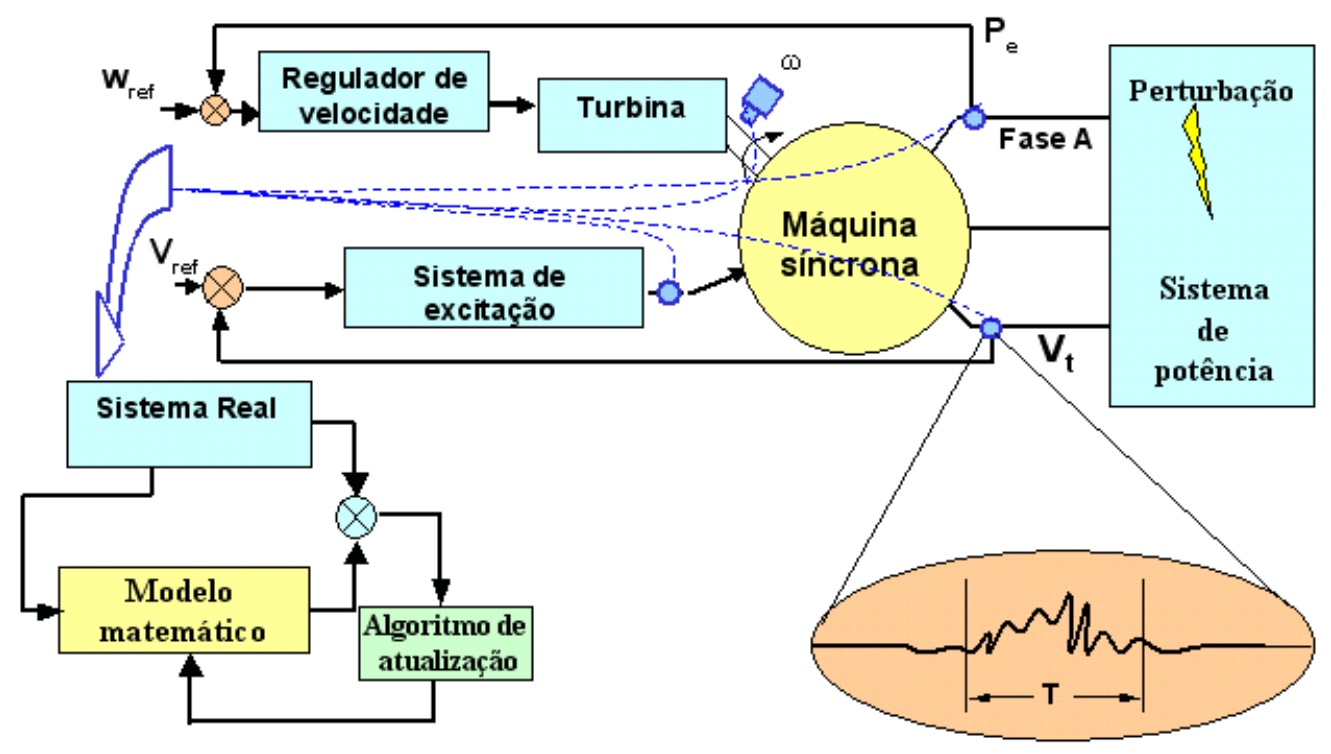

Figura 6.1: Processo de estimação de parâmetros.

Outra consideração é que na presença de uma perturbação no sistema existem três períodos bem definidos: período pré-falta (ou condições de operação normal), período em falta (na presença da perturbação) e período pós-falta (depois de eliminada a perturbação). O processo de estimação é realizado no período pósfalta. Isto pode ser visualizado na figura $(6.2)$. Iniciar a análise após à eliminação da falta não é extremamente necessário mas acreditamos que problemas de ruído e chaveamentos seriam minimizados.

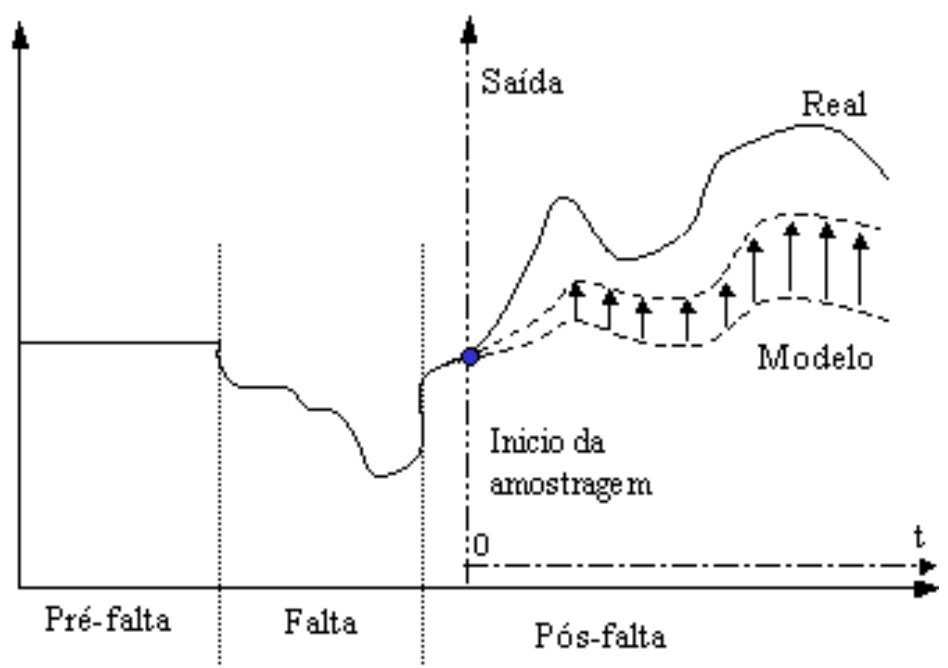

Figura 6.2: Condições de Operação no processo de estimação. 


\subsubsection{Modelo do Sistema de Potência}

O sistema de potência utilizado neste estudo consiste em um gerador de 2200 MVA, $24 \mathrm{kV}$ e $60 \mathrm{~Hz}$ fornecendo potência a um barramento infinito através de duas linhas de transmissão como mostra a figura 6.3. Na referida figura os valores das impedâncias de linha e do transformador estão dados em valores por unidade (pu). A potência base é 2200 MVA, a tensão base no lado de baixa do transformador é $24 \mathrm{kV}$ e no lado de alta é $220 \mathrm{kV}$. O procedimento de estimação de parâmetros aguarda a ocorrência de uma perturbação significativa no sistema de potência. A resposta transitória da máquina síncrona e dos controladores associados são obtidos por intermédio de medidores. A perturbação aplicada consiste em um curto-circuito em um dos terminais da linha de transmissão $\left(L_{2}\right)$. A falta é eliminada pela atuação dos dispositivos de proteção através do isolamento da linha em falta em t $=0.07 \mathrm{~s}$. Em tais condições são amostradas medidas que serão utilizadas pelo procedimento de estimação.

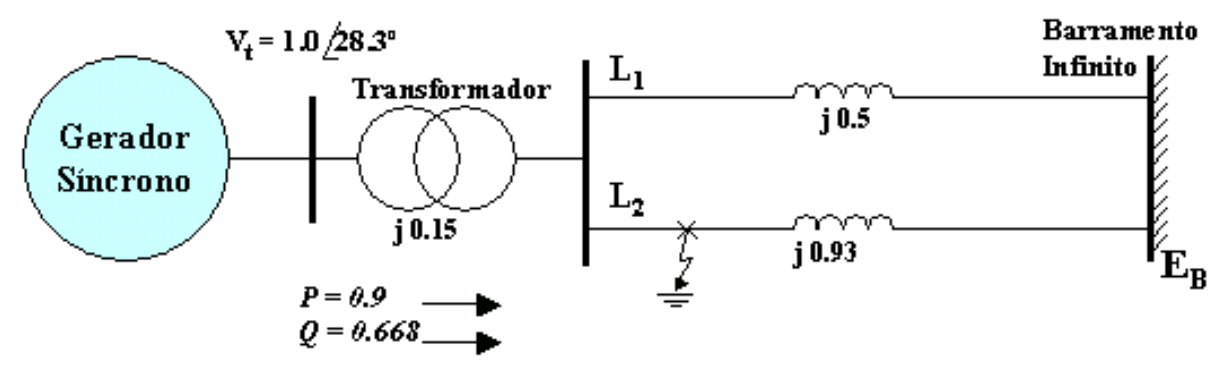

Figura 6.3: Modelo do sistema de potência

\subsection{Estimação de parâmetros da máquina síncrona}

\subsubsection{Parâmetros do modelo clássico}

Inicialmente a máquina síncrona foi representada pelo modelo clássico. Nestes testes os parâmetros a serem determinados são a reatância transitória de eixo direto $x_{d}^{\prime}$ e a constante de inércia $\mathrm{H}$.

Na figura 6.4 mostra-se o circuito equivalente da máquina síncrona na repre- 
sentação clássica. Esta representação considera a máquina como uma fonte de tensão fixa atrás da reatância transitória de eixo direto.

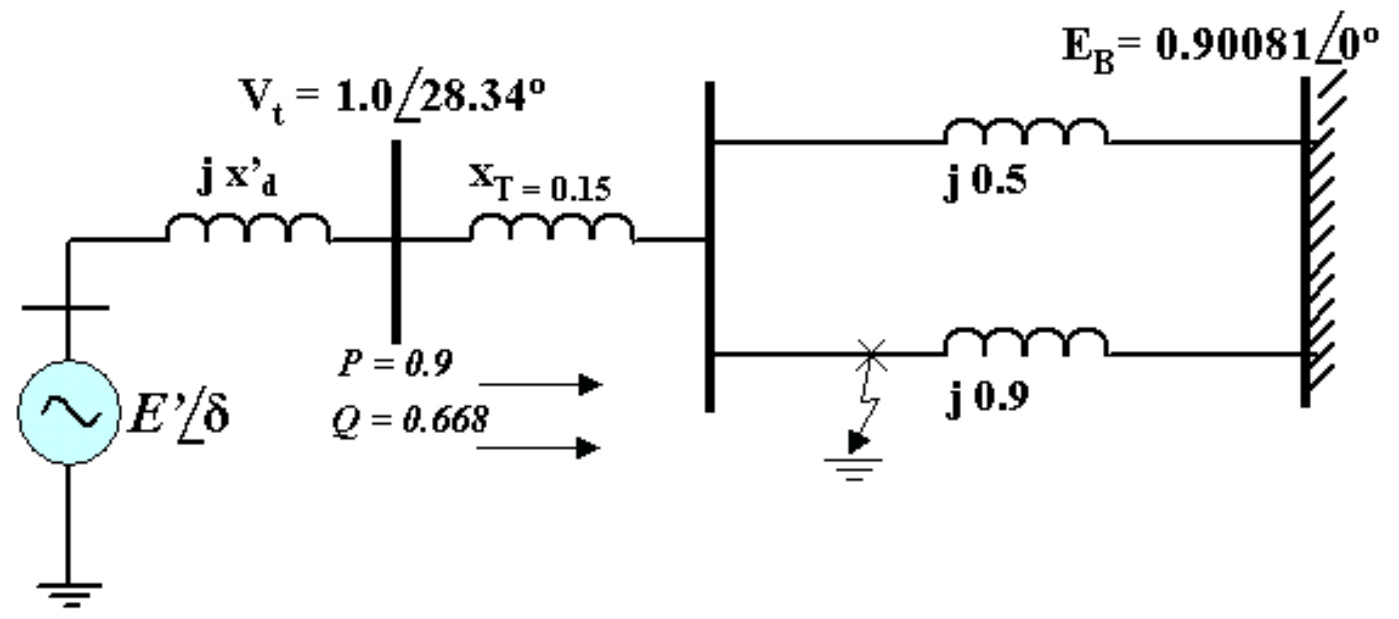

Figura 6.4: Modelo clássico.

As equações para o modelo clássico foram definidas no capítulo 4 e aqui são mostradas novamente para facilitar a análise,

$$
\begin{aligned}
\dot{w} & =\frac{w_{o}}{2 H}\left[P_{m}-\frac{E^{\prime} V_{t}}{x_{d}^{\prime}} \operatorname{sen}(\delta-28.34)\right] \\
\dot{\delta} & =w
\end{aligned}
$$

sendo $w$ a velocidade do rotor (relativa a velocidade síncrona) em rad/s e $\delta$ é o ângulo interno da máquina em radianos.

A primeira equação está em função da tensão interna $E^{\prime}$, que é uma grandeza não mensurável, e do ângulo do rotor $\delta$ que é difícil de ser medido. Nestas condições a metodologia de estimação de parâmetros não pode ser aplicada diretamente às equações 6.2). O projeto de um observador de estados também é inviabilizado pois este ficaria dependente dos parâmetros a serem estimados. Entretanto é possível realizar transformações nas equações originais com o objetivo de viabilizar a aplicação da metodologia.

A referida transformação é obtida a partir da equação da potência aparente nos terminais do gerador. Derivando-se a equação de potência ativa e fazendo algumas substituições de variáveis, encontra-se uma nova equação dinâmica da máquina síncrona para a representação clássica. Os cálculos são detalhados a 
seguir. A potência aparente nos terminais da máquina é dada pela seguinte equação:

$$
\begin{aligned}
& S^{*}=V_{t} * I_{t} \\
& S^{*}=\left(V_{t} \angle \theta\right)^{*} I_{t} \\
& S^{*}=V_{t} \angle-\theta\left(\frac{E^{\prime} \angle \delta-V_{t} \angle \theta}{j x_{d}^{\prime}}\right) \\
& S^{*}=\frac{V_{t} E^{\prime} \angle \delta-\theta}{j x_{d}^{\prime}}-\frac{V_{t}^{2}}{j x_{d}^{\prime}} \\
& S^{*}=-j \frac{V_{t} E^{\prime} \angle \delta-\theta}{x_{d}^{\prime}}+j \frac{V_{t}^{2}}{j x_{d}^{\prime}} \\
& S^{*}=-j \frac{V_{t} E^{\prime}}{x_{d}^{\prime}} \cos (\delta-\theta)+\frac{V_{t} E^{\prime}}{x_{d}^{\prime}} \operatorname{sen}(\delta-\theta)+j \frac{V_{t}^{2}}{x_{d}^{\prime}} \\
& S^{*}=P_{e}-j Q_{e} \\
& P_{e}=\frac{V_{t} E^{\prime}}{x_{d}^{\prime}} \operatorname{sen}(\delta-\theta) \\
& Q_{e}=\frac{-V_{t}^{2}}{x_{d}^{\prime}}+\frac{V t E^{\prime}}{x_{d}^{\prime}} \cos (\delta-\theta)
\end{aligned}
$$

Derivando a equação de potência ativa $\left(P_{e}\right)$ em relação ao tempo tem-se:

$$
\dot{P}_{e}=\dot{V} t \frac{E^{\prime}}{x_{d}^{\prime}} \operatorname{sen}(\delta-\theta)+V t \frac{E^{\prime}}{x_{d}^{\prime}} \cos (\delta-\theta)(\dot{\delta}-\dot{\theta})
$$

Multiplicando e dividindo o primeiro termo da equação (6.5) por $V_{t}$ obtemos:

$$
\dot{P}_{e}=\dot{V}_{t} \frac{V_{t}}{V_{t}} \frac{E^{\prime}}{x_{d}^{\prime}} \operatorname{sen}(\delta-\theta)+V_{t} \frac{E^{\prime}}{x_{d}^{\prime}} \cos (\delta-\theta)(\dot{\delta}-\dot{\theta})
$$

Substituindo $\frac{V_{t} E^{\prime}}{x_{d}^{\prime}} \cos (\delta-\theta)$ por $\left(Q_{e}+\frac{V_{t}^{2}}{x_{d}^{\prime}}\right)$ e $\dot{\theta}$ por $\omega$ tem-se

$$
\dot{P}_{e}=\frac{\dot{V}_{t}}{V_{t}} P_{e}+\left(Q_{e}+\frac{V_{t}^{2}}{x_{d}^{\prime}}\right)(\omega-\dot{\theta})
$$

Esta nova equação está em função de $P_{e}, Q_{e}, \omega, V_{t}$, que são, em princípio, mensuráveis. Ficando apenas a medida de $\dot{\theta}$ para ser obtida do sistema real. As derivadas $\dot{V}_{t}$ e $\dot{\theta}$ podem ser calculados a partir das seguintes aproximações:

$\dot{V}_{t}=\frac{\left.V_{t}(k+1)-V t(k)\right)}{\Delta t}$ e $\dot{\theta}_{t}=\frac{\theta(k+1)-\theta(k))}{\Delta t}$. Aproximações similares para medidas de corrente foram aplicadas em (Kyriakides et al., 2004) de forma satisfatória. 
A segunda equação permanece inalterada, e é dada por:

$$
\dot{w}=\frac{\omega_{o}}{2 H}\left(P_{m}-P_{e}\right)
$$

A mudança realizada pode ser visualizada na figura (6.5). As variáveis de estado das novas equações são a potência $\left(P_{e}\right)$ e a velocidade angular $(\omega)$.

$$
\begin{gathered}
\text { Original } \\
\dot{\delta}=\omega \\
\dot{\omega}=\frac{\omega_{0}}{2 \mathrm{H}}\left(P_{m}-P_{e}\right)
\end{gathered} \mid \begin{aligned}
& \dot{\mathrm{P}}_{\mathrm{e}}=\frac{\dot{\mathrm{V}}_{\mathrm{t}}}{\mathrm{V}_{\mathrm{t}}} \mathrm{P}_{\mathrm{e}}+\left(\mathrm{Q}_{\mathrm{e}}+\frac{\mathrm{V}_{\mathrm{t}}^{2}}{x_{d}^{\prime}}\right)(\omega-\dot{\theta}) \\
& \dot{\omega}=\frac{\omega_{o}}{2 \mathrm{H}}\left(\mathrm{P}_{\mathrm{m}}-\mathrm{P}_{\mathrm{e}}\right)
\end{aligned}
$$

Figura 6.5: Transformação das equações originais do modelo clássico.

Na etapa seguinte do processo de estimação é necessário determinar as variáveis de entrada e as variáveis de saída. Neste testes escolheram-se como variáveis de entrada $\left(Q_{e}, V_{t}, \dot{V}_{t}, \dot{\theta}\right)$, e como variável de saída $P_{e}$.

Na figura (6.6), mostram-se as entradas e as saídas escolhidas para a determinação dos parâmetros $\mathrm{H}$ e $x_{d}^{\prime}$. O sub-índice ' $r$ ' denota variáveis do 'sistema real'. 


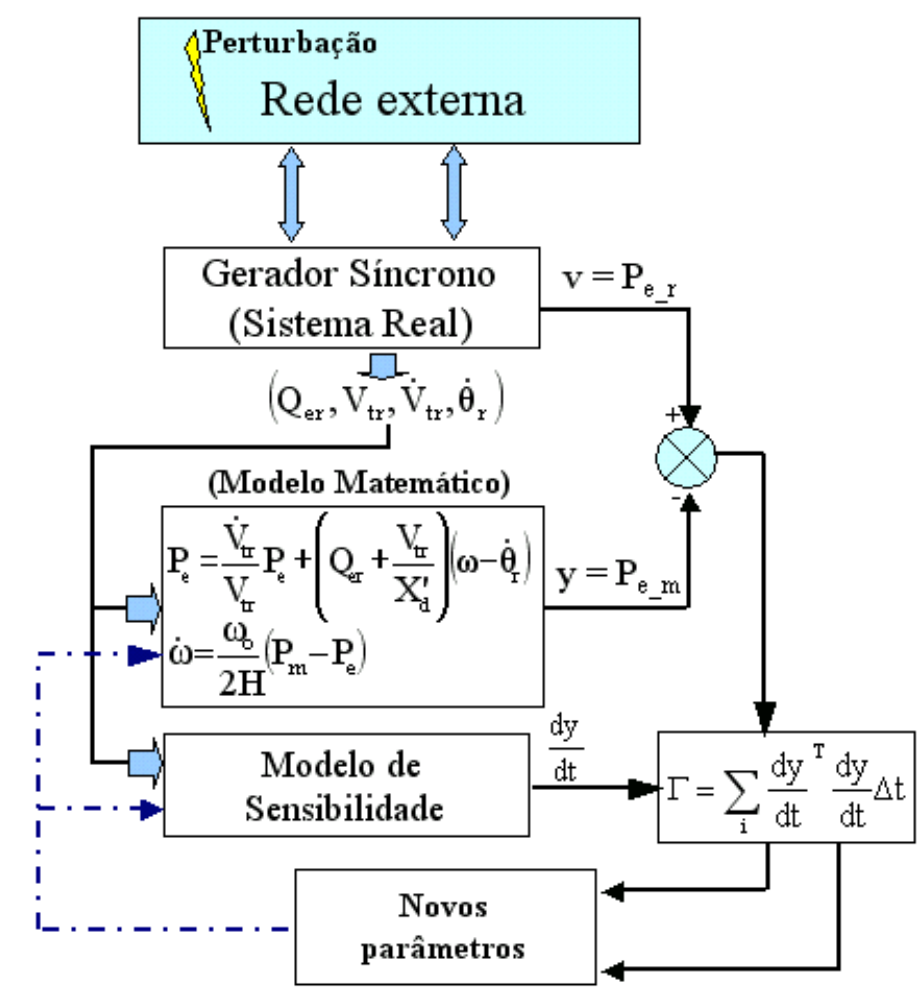

Figura 6.6: Diagrama esquemático da aplicação da metodologia de estimação de parâmetros para o modelo clássico.

onde:

- y : saída do modelo matemático.

- v : saída do sistema real

O bloco 'modelo de sensibilidade' da figura 6.6 contém as funções de sensibilidade as quais são calculadas derivando as equações (6.7) e (6.8) em relação aos parâmetros $\mathrm{H}$ e $x_{d}^{\prime}$. As derivadas das referidas equações são:

$$
\left(\begin{array}{l}
\dot{\lambda}_{\delta}^{H} \\
\dot{\lambda_{\omega}^{H}} \\
\dot{\lambda}_{\delta}^{x_{d}^{\prime}} \\
\dot{\lambda}_{\omega}^{x_{d}^{\prime}}
\end{array}\right)=\left(\begin{array}{c}
\frac{\dot{V}_{t}}{V_{t}} \lambda_{\delta}^{H}+\left(Q_{e}+\frac{V_{t}^{2}}{X_{d}^{\prime}}\right) \lambda_{\omega}^{H} \\
\frac{-w_{o}}{2 H^{2}}\left(P_{m}-P_{e}\right)-\frac{w_{o}}{2 H} \lambda_{\delta}^{H} \\
\dot{V_{t}} \lambda_{\delta}^{x_{d}^{\prime}}-\frac{V_{t}^{2}}{x_{d}^{\prime 2}}(w-\dot{\theta})+\left(Q_{e}+\frac{V_{t}^{2}}{x_{d}^{\prime}}\right) \lambda_{\omega}^{x_{d}^{\prime}} \\
\frac{-w_{o}}{2 H} \lambda_{\delta}^{x_{d}^{\prime}}
\end{array}\right)
$$

onde $\lambda_{i}^{k}=\frac{\partial y_{i}}{\partial p_{i}}$ representa a sensibilidade da função i em relação ao parâmetro k. 
Aplica-se uma perturbação no sistema no tempo $t=0$ para obter a resposta transitória do gerador. A perturbação é uma falta trifásica na linha 2 (L-2) próxima à barra 1. A falta é eliminada pela atuação dos dispositivos de proteção no tempo $\mathrm{t}=0.07 \mathrm{~s}$ isolando a linha em falta.

Nestas condições, a metodologia estimou os parâmetros com erro inferior a $3 \%$. A potência elétrica e a velocidade angular do 'modelo matemático' e do 'sistema real' antes e após a estimação dos parâmetros são mostrados respectivamente nas figuras 6.7) e 6.8.
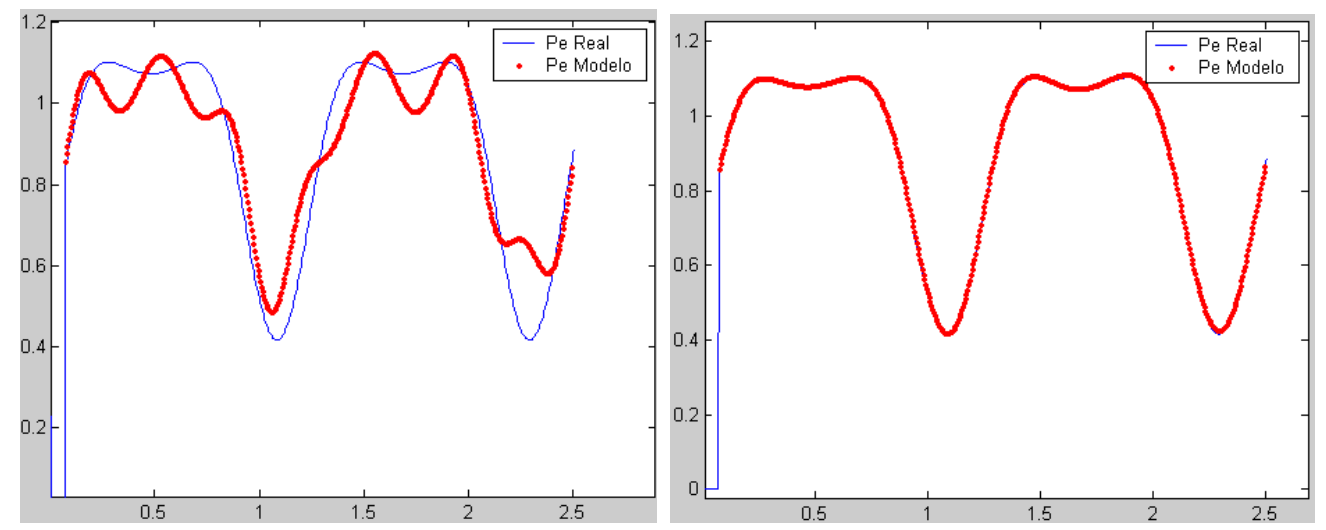

Figura 6.7: Comparação da potência elétrica antes e depois a estimação dos parâmetro para o 'modelo clássico'
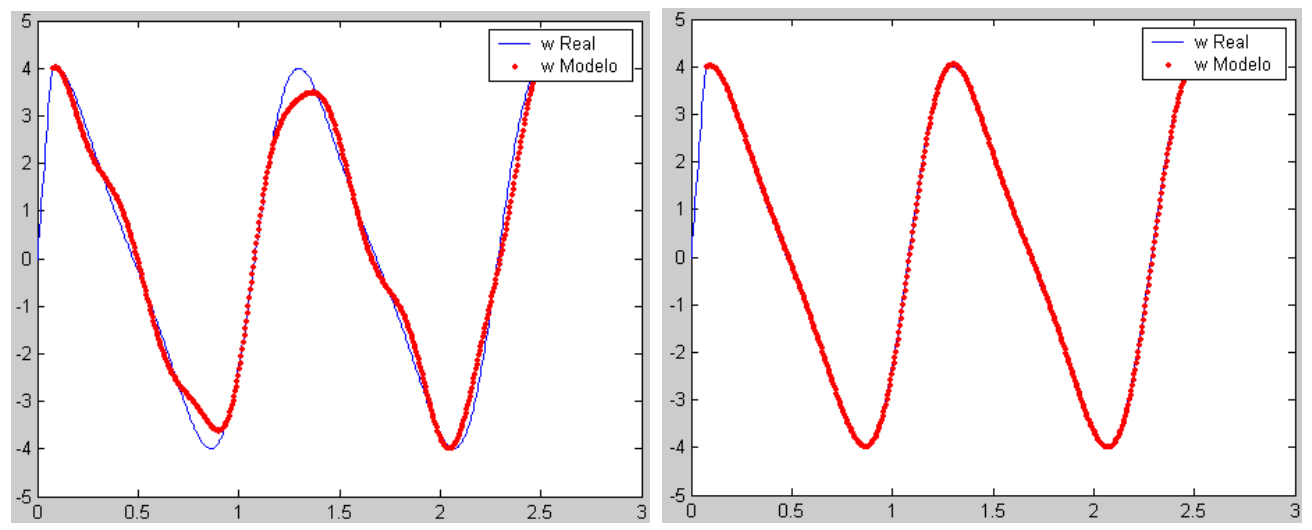

Figura 6.8: Velocidade angular antes e depois da estimação dos parâmetros para o 'modelo clássico'.

Na tabela 6.1 mostra-se um resumo dos resultados obtidos com o processo de estimação. O passo de integração utilizado (equivalente a freqüência de amostragem) 
é de 5 ms. Os parâmetros foram estimados após 24 iterações.

Tabela 6.1: Estimação dos parâmetros do modelo clássico.

\begin{tabular}{|c|c|c|c|c|}
\hline parâmetro & V. Inicial & V. Final & V. Verdadeiro & Erro(\%) \\
\hline H & 2.4 & 2.9864 & 3 & -0.45 \\
\hline$x_{d}^{\prime}$ & 0.24 & 0.3061 & 0.3 & 2.03 \\
\hline
\end{tabular}

Algumas dificuldades de convergência foram encontradas ao considerar a potência elétrica $\left(P_{e}\right)$ e a velocidade angular $(w)$ como variáveis de saída. Entretanto considerando apenas uma saída $P_{e}$ ou $w$ os parâmetros foram estimados de forma satisfatória. A explicação é que ambas as saídas apresentam dependência linear.

\subsubsection{Estimação dos parâmetros do modelo de 'um eixo'}

As equações para o modelo de um eixo foram apresentadas no capítulo quatro subseção 5.1.5, aqui são mostradas novamente para facilitar a análise. Nestas equações as correntes no $I_{d}$ e $I_{q}$ foram substituídas em função dos parâmetros da rede.

$$
\begin{aligned}
\dot{\delta}_{1} & =w_{1} \\
\dot{w}_{1} & =\frac{w_{o}}{2 H}\left[P_{m}-\frac{D}{w_{o}} w-E_{q 1}^{\prime} E_{q 2}^{\prime} B_{12} \operatorname{sen}\left(\delta_{i}\right)\right] \\
\dot{E_{q 1}^{\prime}} & =\frac{1}{T_{d o}^{\prime}}\left[E_{f d}-E_{q 1}^{\prime}+\left(x_{d}-x_{d}^{\prime}\right)\left(B_{11} E_{q 1}^{\prime}+B_{12} E_{q 2}^{\prime} \cos \left(\delta_{1}\right)\right)\right] \\
\dot{E}_{f d} & =\frac{1}{T_{R}}\left(K_{A}\left(V_{\text {ref }}-V_{t}\right)-E_{f d}\right)
\end{aligned}
$$

sendo:

- $E_{q 2}$ : tensão no barramento infinito.

- $E_{q 1}$ : a tensão equivalente de eixo em quadratura do gerador.

- $B_{11}$ : a suceptância própria da rede extraída da $Y_{B u s}$ reduzida.

- $B_{12}$ : a suceptância de transferência da rede extraída da $Y_{B u s}$ reduzida. 
Estas equações consideram a aproximação $x_{d}^{\prime}=x_{q}^{\prime}$ permitindo representar o modelo da máquina por uma fonte variável $E_{q}^{\prime}$ atrás de uma reatância transitória de eixo direto. O qual é similar ao sistema da figura 6.4 apenas diferenciando-se pela tensão interna que agora varia em cada instante do tempo.

Os parâmetros $B_{11}$ e $B_{12}$ são obtidos a partir da matriz $Y_{B u s}$ da rede reduzida aos barramentos terminais da máquina tanto em regime de falta como em pósfalta como mostra a figura 6.9 .

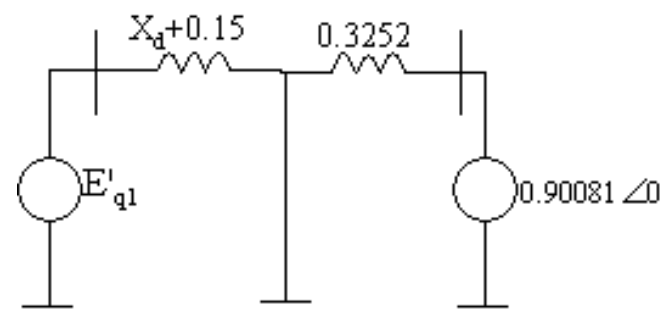

(a) Sistema em falta

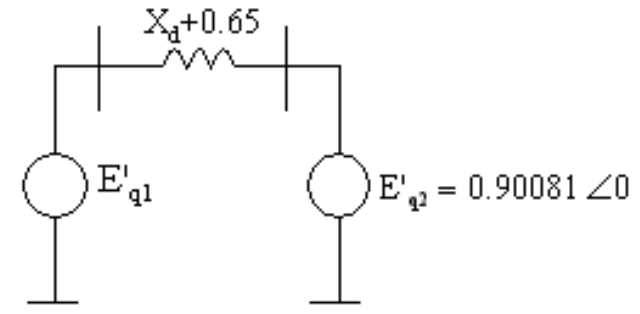

(b) Sistema em pós-falta

Figura 6.9: Sistema de potência reduzido aos barramentos terminais da máquina em falta e pós-falta.

A matriz $Y_{B u s}$ em falta é dada por,

$$
Y_{\text {bus }}=\left[\begin{array}{cc}
\frac{-1}{x_{d}^{\prime}+0.15} & 0 \\
0 & -3.075
\end{array}\right]
$$

e a matriz $Y_{\text {Bus }}$ em pós-falta é dada por:

$$
Y_{\text {bus }}=\left[\begin{array}{cc}
\frac{-1}{x_{d}^{\prime}+0.65} & \frac{1}{x_{d}^{\prime}+0.65} \\
\frac{1}{x_{d}^{\prime}+0.65} & \frac{-1}{x_{d}^{\prime}+0.65}
\end{array}\right]
$$

Assim:

$$
\begin{aligned}
\frac{\partial B_{11}}{\partial x_{d}^{\prime}} & =\frac{1}{\left(x_{d}^{\prime}+0.65\right)^{2}} \\
\frac{\partial B_{12}}{\partial x_{d}^{\prime}} & =\frac{-1}{\left(x_{d}^{\prime}+0.65\right)^{2}}
\end{aligned}
$$

O vetor de parâmetros a serem determinados neste teste é dado por: $p=\left[\begin{array}{llll}H, & T_{d o}^{\prime}, & x_{d}, & x_{d}^{\prime}\end{array}\right]^{t}$. 
Em $t=0$ as condições iniciais são:

$$
\left[\begin{array}{c}
\delta\left(t_{o}\right) \\
w\left(t_{o}\right) \\
E_{q o}^{\prime}\left(t_{o}\right) \\
E_{f d}\left(t_{o}\right)
\end{array}\right]=\left[\begin{array}{c}
41.77^{\circ} \\
0 \\
1.1626 \\
1.4517
\end{array}\right]
$$

A tensão terminal foi considerada como variável de entrada neste teste. No diagrama esquemático da figura 6.10 mostram-se as entradas e saídas do 'modelo matemático' e do 'sistema real'.

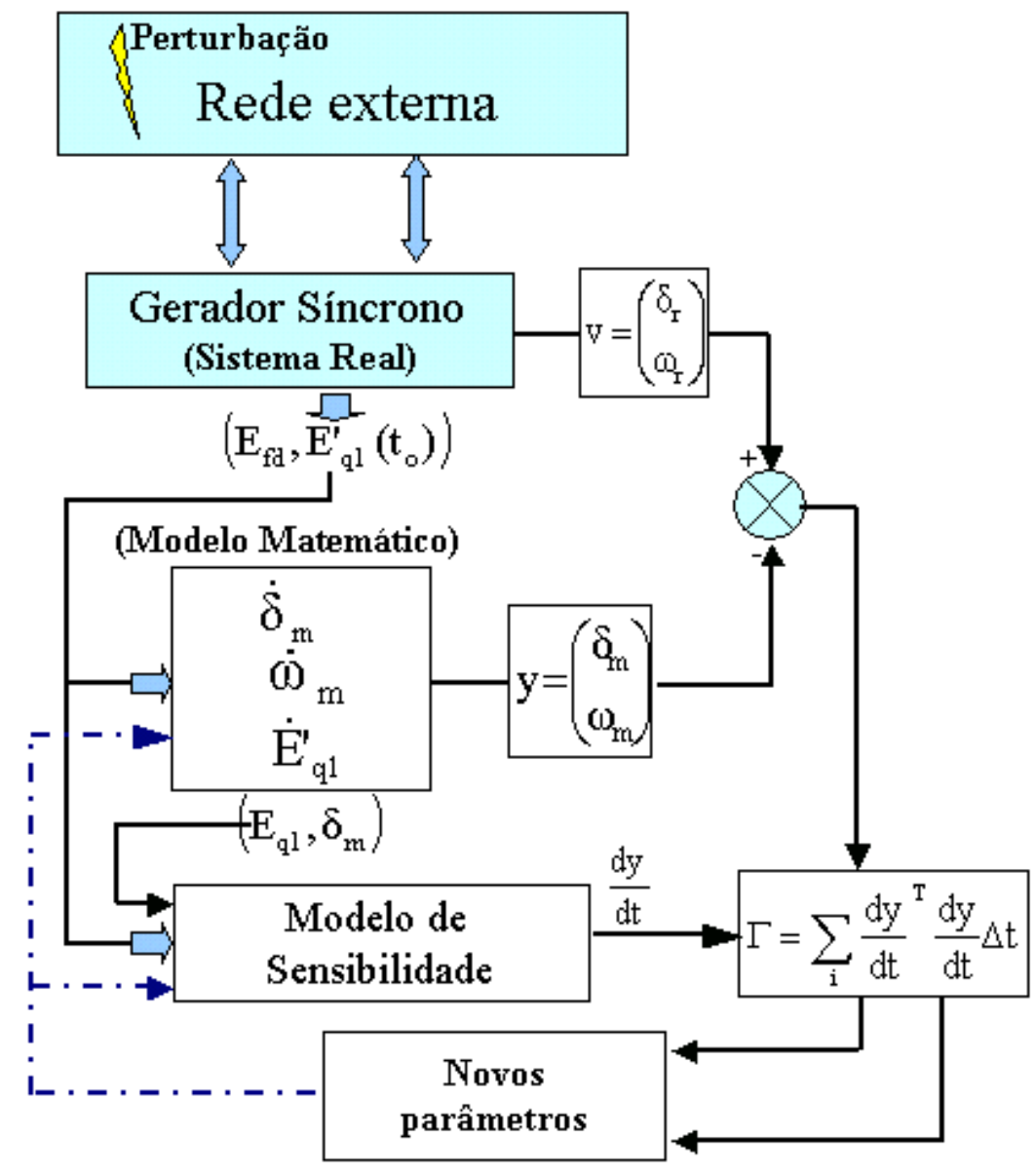

Figura 6.10: Variáveis de entrada e saída para o modelo de 'um eixo'.

O 'modelo matemático' considera apenas três equações da máquina síncrona $\delta_{m}, w_{m}$ e $E_{q 1}^{\prime}$. A equação da tensão do sistema de excitação não é considerada no modelo matemático porque a variável $E_{f d}$ é considerada como entrada no mesmo. Além disso, a tensão no eixo em quadratura no instante $\left(t_{o}\right)$ é considerada como 
um dado que pode ser medido no sistema real (mais adiante, realizar-se-á outro teste considerando este valor como parâmetro). As saídas escolhidas para este teste foram o ângulo interno e a velocidade do rotor da máquina síncrona. $\mathrm{O}$ sub-índice 'm' denota variável do 'modelo matemático' e o sub-índice 'r' denota variável do 'sistema real'.

As funções de sensibilidade são calculadas derivando as equações diferenciais 6.9 com relação aos parâmetros e integrado-as no tempo. Denotando $\lambda_{f i}^{p}=\frac{\partial f i}{\partial p}$ como a sensibilidade da função ' $f_{i}^{\prime}$ em relação ao parâmetro p, (fi=1,2,3 corresponde a $\delta_{m}, w_{m}, E_{q m}^{\prime}$, respectivamente).

Derivando-se o conjunto de equações 6.9 em relação a $x_{d}$ tem-se:

$$
\begin{array}{ll}
\dot{\lambda}_{1}^{x_{d}}= & \lambda_{2}^{x_{d}} \\
\dot{\lambda}_{2}^{x_{d}}= & \frac{w_{o}}{2 H}\left(-\frac{D}{w_{o}} \lambda_{2}^{x_{d}}-E_{q 2}^{\prime} B_{12}\left[\lambda_{3}^{x_{d}} \operatorname{sen}\left(\delta_{m}\right)+x_{3} \cos (\delta) \lambda_{1}^{x_{d}}\right]\right) \\
\dot{\lambda}_{3}^{x_{d}}= & \frac{1}{T_{d o}^{\prime}}\left(-\lambda_{3}^{x_{d}}+\left[B_{11} E_{q 1}^{\prime}+B_{12} E_{q 2}^{\prime} \cos \left(\delta_{m}\right)\right]+\left(x_{d}-x_{d}^{\prime}\right)\right. \\
& \left.\left[B_{11} \lambda_{3}^{x_{d}}-B_{12} E_{q 2}^{\prime} \operatorname{sen}\left(\delta_{m}\right) \lambda_{1}^{x_{d}}\right]\right)
\end{array}
$$

Derivando-se o conjunto de equações (6.9) em relação a $x_{d}^{\prime}$ tem-se:

$$
\begin{aligned}
\dot{\lambda}_{1}^{x_{d}^{\prime}}= & \lambda_{2}^{x_{d}^{\prime}} \\
\dot{\lambda}_{2}^{x_{d}^{\prime}}= & \frac{w_{o}}{2 H}\left(-\frac{D}{w_{o}} \lambda_{2}^{x_{d}^{\prime}}-E_{q 2}^{\prime}\left[\lambda_{3}^{x_{d}^{\prime}} B_{12} \operatorname{sen}\left(\delta_{m}\right)+E_{q 1}^{\prime} \frac{\partial B_{12}}{\partial x_{d}^{\prime}} \sin \left(\delta_{m}\right)+E_{q 1} B_{12} \cos \left(\delta_{m}\right) \lambda_{1}^{x_{d}^{\prime}}\right]\right) \\
\dot{\lambda}_{3}^{x_{d}^{\prime}}= & \frac{1}{T_{d o}^{\prime}}\left(-\lambda_{3}^{x_{d}^{\prime}}-\left[B_{11} E_{q 1}^{\prime}+B_{12} E_{q 2}^{\prime} \cos \left(\delta_{m}\right)\right]+\left(x_{d}-x_{d}^{\prime}\right)\left[\frac{\partial B_{11}}{\partial x_{d}^{\prime}} E_{q 1}^{\prime}+B_{11} \lambda_{3}^{x_{d}^{\prime}}+\right.\right. \\
& \left.\left.E_{q 2}^{\prime}\left(\frac{\partial B_{12}}{\partial x_{d}^{\prime}} \cos \left(\delta_{m}\right)-B_{12} \operatorname{sen}\left(\delta_{m}\right) \lambda_{1}^{x^{\prime} d}\right)\right]\right)
\end{aligned}
$$

Derivando-se o conjunto de equações 6.9 em relação a H tem-se:

$$
\begin{aligned}
\dot{\lambda}_{1}^{H}= & \lambda_{2}^{H} \\
\dot{\lambda}_{2}^{H}= & \frac{-w_{o}}{H^{2}}\left(P_{m}-\frac{D}{w_{o}} w_{m}-E_{q 1}^{\prime} E_{q 2}^{\prime} B_{12} \operatorname{sen}\left(\delta_{m}\right)\right)+\frac{w_{o}}{2 H}\left(-D \lambda_{2}^{H}-\right. \\
& \left.\lambda_{3}^{H} E_{q 2}^{\prime} B_{12} \operatorname{sen}\left(\delta_{m}\right)-E_{q 1}^{\prime} E_{q 2}^{\prime} B_{12} \cos \left(\delta_{m}\right) \lambda_{1}^{H}\right) \\
\dot{\lambda}_{3}^{x_{d}^{\prime}}= & \frac{1}{T_{d o}^{\prime}}\left(-\lambda_{3}^{H}+\left(x_{d}-x_{d}^{\prime}\right)\left[B_{11} \lambda_{3}^{H}-B_{12} E_{q 2}^{\prime} \operatorname{sen}\left(\delta_{m}\right) \lambda_{1}^{H}\right]\right)
\end{aligned}
$$


Derivando-se o conjunto de equações (6.9) em relação a $T_{d 0}^{\prime}$ tem-se:

$$
\begin{aligned}
\dot{\lambda}_{1}^{T_{d o}}= & \lambda_{2}^{T_{d o}^{\prime}} \\
\dot{\lambda}_{2}^{T_{d o}^{\prime}}= & \frac{w_{o}}{2 H}\left(-\frac{D}{w_{o}} \lambda_{2}^{T_{d o}^{\prime}}-E_{q 2}^{\prime} B_{12}\left[\lambda_{3}^{T_{d o}^{\prime}} \operatorname{sen}\left(\delta_{m}\right)+E_{q 1}^{\prime} \cos \left(\delta_{m}\right) \lambda_{1}^{T_{d o}^{\prime}}\right]\right) \\
\dot{\lambda}_{3}^{T_{d o}^{\prime}}= & \frac{-1}{T_{d o}^{\prime 2}}\left(E_{f d}-E_{q 1}^{\prime}+\left(x_{d}-x_{d}^{\prime}\right)\left[B_{11} E_{q 1}^{\prime}+B_{12} E_{q 2}^{\prime} \cos \left(\delta_{m}\right)\right]\right)+ \\
& \frac{1}{T_{d o}^{\prime}}\left(-\lambda_{3}^{T_{d o}^{\prime}}+\left(x_{d}-x_{d}^{\prime}\right)\left(B_{11} \lambda_{3}^{T_{d o}^{\prime}}-B_{12} E_{q 2}^{\prime} \operatorname{sen}\left(\delta_{m}\right) \lambda_{1}^{T_{d o}^{\prime}}\right)\right)
\end{aligned}
$$

Os valores iniciais dos parâmetros do modelo matemático são obtidos alterando os parâmetros verdadeiros em $+20 \%$ e $-20 \%$. Após 8 iterações os parâmetros foram estimados satisfatoriamente. Nas figuras 6.11 e 6.12 são apresentadas as saídas do ângulo e da velocidade do rotor da máquina síncrona respectivamente, antes e depois do processo de estimação.
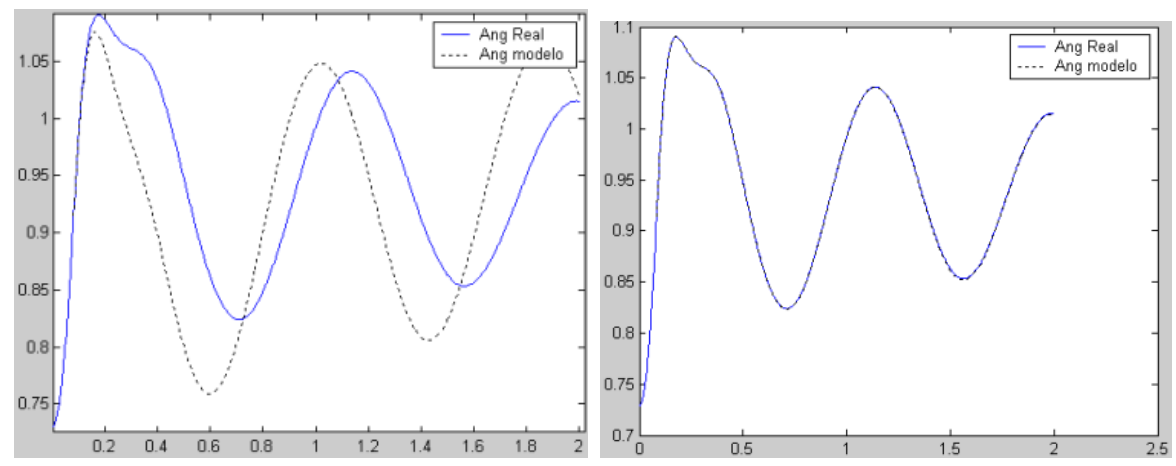

Figura 6.11: Ângulo do rotor, antes e depois da estimação.
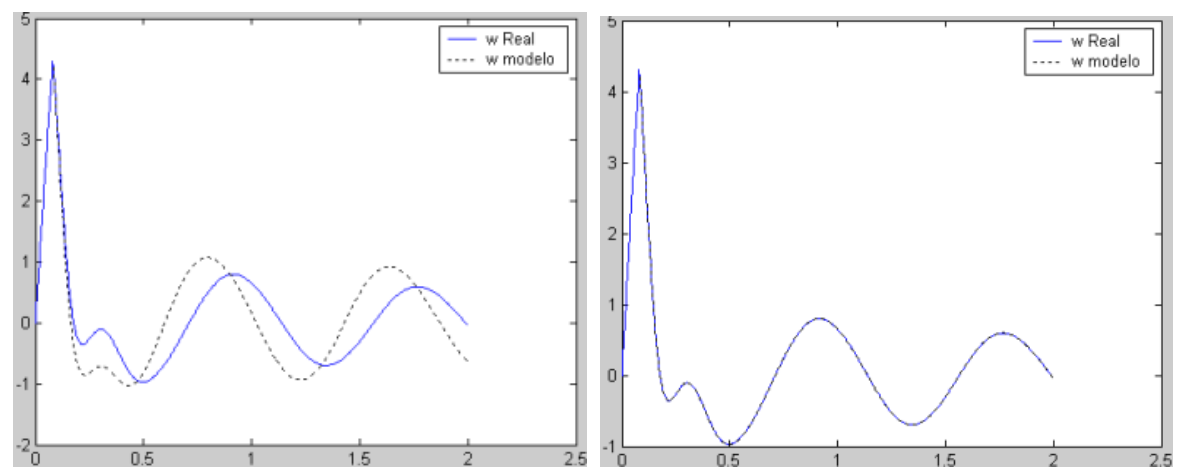

Figura 6.12: Velocidade angular antes e depois da estimação dos parâmetros para o modelo de um eixo 
Os erros do processo de estimação são inferiores a $0.3 \%$ em todos os casos conforme mostra a tabela 6.2 .

Tabela 6.2: Estimação dos parâmetros do modelo de 'um eixo'.

\begin{tabular}{|c|c|c|c|c|}
\hline parâmetros & V. Inicial & V. Final & V. Verdadeiro & Erro(\%) \\
\hline$x_{d}$ & 1.26 & 1.0476 & 1.05 & 0.229 \\
\hline$x_{d}^{\prime}$ & 0.36 & 0.3004 & 0.3 & -0.133 \\
\hline $\mathrm{H}$ & 2.4 & 2.9987 & 3 & 0.043 \\
\hline$T_{d o}^{\prime}$ & 6 & 5.0015 & 5 & -0.03 \\
\hline
\end{tabular}

As funções de sensibilidade das saídas em relação aos parâmetros são mostradas na figura 6.13. A função de sensibilidade da saída com relação ao parâmetro $x_{d}^{\prime}$ tem uma influência maior na estimação dos parâmetros. Observa-se que a função de sensibilidade da constante de tempo transitória $T_{d o}^{\prime}$ é contrária àquela da $x_{d}^{\prime}$, como mostra a figura 6.13 .
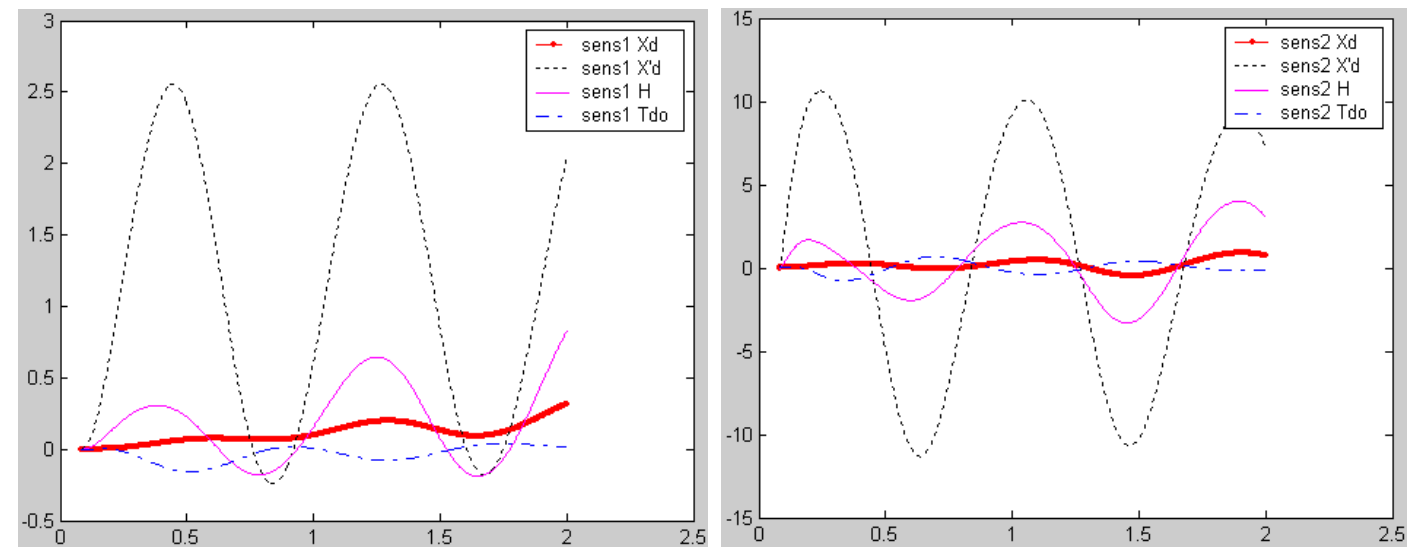

Figura 6.13: Sensibilidades das saídas 1 e 2 com relação aos parâmetros.

Realizaram-se vários testes verificando a robustez 1 do método para a estimação dos parâmetros. Os parâmetros foram estimados satisfatoriamente em situações onde os parâmetros iniciais estão distantes dos valores verdadeiros em até $25 \%$. Entretanto, pelo fato de que as funções de sensibilidades de $x_{d}^{\prime}$ e $T_{d o}^{\prime}$ serem contrárias (enquanto um é máximo o outro é mínimo e vice-versa), existem direções nas quais isto não é verdade como, por exemplo, para uma variação

\footnotetext{
${ }^{1}$ em relação aos valores inicias dos parâmetros
} 
de $-17 \%$ de $T_{d o}$ e $20 \%$ de $x_{d}^{\prime}$ em relação aos valores verdadeiros, o procedimento diverge. Na figura (6.14) mostra-se a sensibilidade da saída 1 em relação aos parâmetros. Pode-se observar, na referida figura, que as duas sensibilidade em relação ao parâmetro 'H' explodem dando um claro indicativo da não convergência do procedimento de estimação de parâmetros ao utilizar os valores mencionados como parâmetros iniciais.
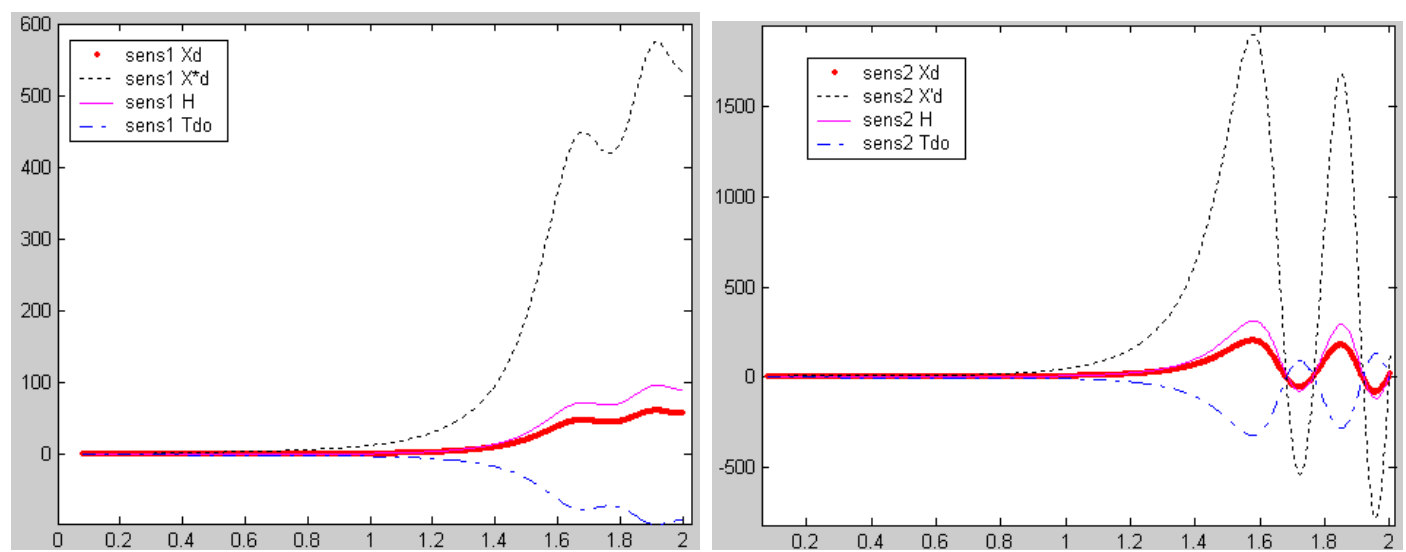

Figura 6.14: Sensibilidades das saídas 1 e 2 respeito aos parâmetros, caso de não convergência.

\section{Estimação dos parâmetros do modelo de 'um eixo' considerando $E_{q o}^{\prime}$ como parâmetro}

A implementação computacional anterior não considera aspectos relacionados à implementação prática da metodologia. Entre as dificuldades para a implementação relacionada a este modelo podemos citar:

- O estado $E_{q}^{\prime}$ é uma variável não mensurável.

- O equacionamento do modelo desenvolvido está em função da barra infinita (valor que é difícil de se estimar), o ideal seria considerar apenas as variáveis próprias do gerador em análise (tensão terminal, correntes, potência, etc).

- O equacionamento inclui as reatâncias da rede (as quais foram incorporadas as equações do gerador), estas reatâncias estão sujeitas a erros os quais são incorporados no processo de estimação de parâmetros do gerador síncrono.

No próximo teste, a dinâmica de $E_{q}^{\prime}$ foi considerada como uma variável interna 
do sistema, para isto, a variável inicial $E_{q o}^{\prime}$ foi considerada como um parâmetro a ser determinado pela metodologia.

As funções de sensibilidade em relação a $E_{q o}^{\prime}$ são as seguintes:

$$
\begin{aligned}
& \dot{\lambda}_{1}^{E_{q o}^{\prime}}=\lambda_{2}^{E_{q o}^{\prime}} \\
& \dot{\lambda}_{2}^{E_{q o}^{\prime}}=\frac{w_{o}}{2 H}\left(-\frac{D}{w_{o}} \lambda_{2}^{E_{q o}^{\prime}}-E_{q 2}^{\prime} B_{12}\left[\lambda_{3}^{E_{q o}^{\prime}} \operatorname{sen}\left(\delta_{m}\right)+E_{q 1}^{\prime} \cos \left(\delta_{m}\right) \lambda_{1}^{E_{q o}^{\prime}}\right]\right) \\
& \dot{\lambda}_{3}^{E_{q o}^{\prime}}=\frac{1}{T_{q o}^{\prime}}\left(-\lambda_{3}^{E_{q o}^{\prime}}-\left(x_{d}-x_{d}^{\prime}\right)\left[B_{11} \lambda_{3}^{E_{q o}^{\prime}}-B_{12} E_{q 2}^{\prime} \operatorname{sen}\left(\delta_{m}\right) \lambda_{1}^{E_{q o}^{\prime}}\right]\right)
\end{aligned}
$$

Inicialmente tentou-se estimar este novo parâmetro conjuntamente com outros parâmetros do modelo de 1 eixo (os parâmetros foram perturbados entre 15 e 20 \% do valor verdadeiro). Infelizmente não foi possível estimar os cinco parâmetros ao mesmo tempo, pois o sistema é muito sensível a incerteza do parâmetro $E_{q o}^{\prime}$. Com uma variação de $20 \%$ de $E_{q o}^{\prime}$ a saída do modelo matemático se distancia muito da saída do sistema real e o processo não converge. Para contornar este inconveniente modificou-se o teste da seguinte maneira:

- Em primeiro lugar, os parâmetros do modelo clássico $x_{d}^{\prime}$ e H foram estimados através da representação do modelo clássico.

- Aplicou-se a técnica, numa primeira fase ( $1^{a}$ fase), para obter uma estimativa melhor do parâmetro $E_{q o}^{\prime}$ mantendo os outros parâmetros fixos . Na figura 6.16 mostram-se algumas curvas ao final da primeira fase.

- Aplicou-se a metodologia para estimar simultaneamente $E_{q o}^{\prime}, x_{d}, T_{d o}^{\prime}\left(2^{a}\right.$ fase). Na figura 6.17 mostram-se algumas curvas ao final da segunda fase.
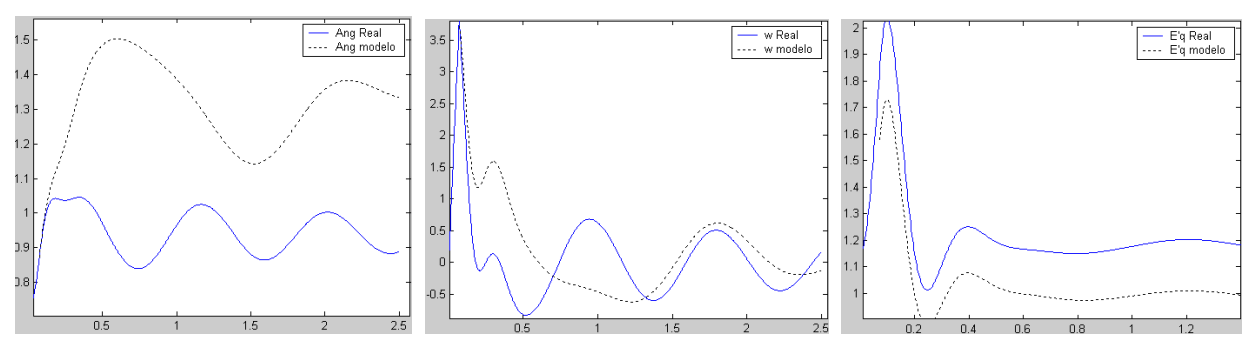

Figura 6.15: ângulo(ang), velocidade $(w)$, e $E_{q}^{\prime}$ antes da estimação dos parâmetros. 

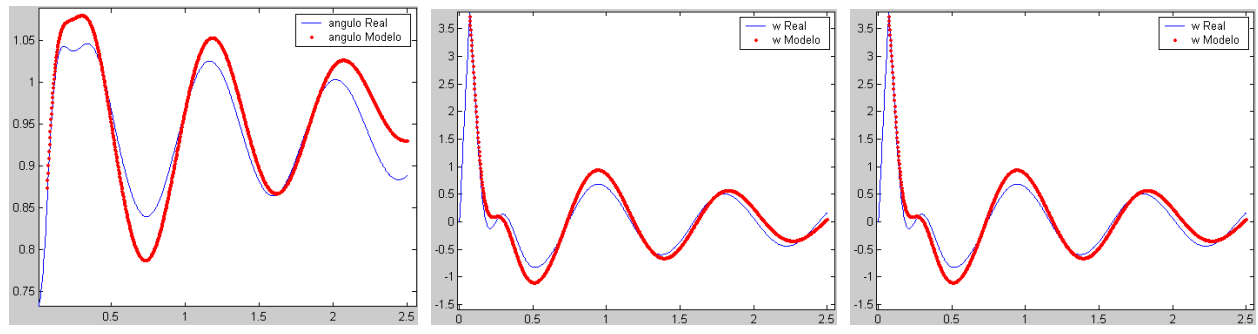

Figura 6.16: ângulo(ang), velocidade $(w)$, e $E_{q}^{\prime}$ ao final da $1^{r a}$ fase.
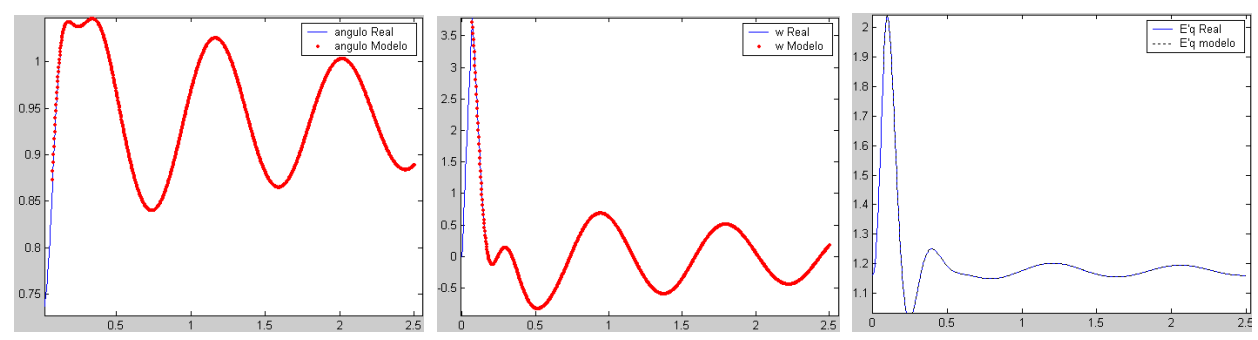

Figura 6.17: ângulo(ang), velocidade $(w)$, e $E_{q}^{\prime}$ ao final da $2^{d a}$ fase.

As entradas e saídas deste testes são as mesmas que as do teste anterior (vide figura 6.10). Segundo estes procedimento, os parâmetros foram estimados com uma boa aproximação (6.17). As condições para a perturbação são as mesmas utilizadas no caso anterior. O período de amostragem está entre 0.09 e $2.5 \mathrm{~s}$. Na tabela 6.3 são apresentados os resultados obtidos. Os parâmetros ' $H$ 'e ' $x_{d}^{\prime}$ ' foram obtidos a partir da tabela 6.1.

Tabela 6.3: Estimação dos parâmetros do modelo de 'um eixo' incluindo $E_{q o}^{\prime}$.

\begin{tabular}{|c|c|c|c|c|c|}
\hline parâmetro & V. Inicial & $1^{r a}$ Fase & $2^{d a}$ Fase (final) & V. Real & Erro(\%) \\
\hline$H$ & 2.9864 & $\ldots$ & 2.9864 & 3 & -0.45 \\
\hline$x_{d}^{\prime}$ & 0.3061 & $\ldots$ & 0.3061 & 0.3 & 2.03 \\
\hline$x_{d}$ & 1.26 & $\ldots$ & 1.0499 & 1.05 & 0 \\
\hline$T_{d o}^{\prime}$ & 6 & $\ldots$ & 5.0005 & 5 & 0.01 \\
\hline$E_{q o}^{\prime}$ & 1.5794 & 1.7727 & 1.8579 & 1.8581 & -0.01 \\
\hline
\end{tabular}




\subsection{Determinação dos parâmetros do modelo de dois eixos}

Nesta seção apresentam-se alguns testes realizados para a estimação de parâmetros da máquina síncrona através da representação do modelo de dois eixos.

Vários testes foram realizadas na tentativa de resolver os problemas deste modelo.

\subsubsection{Redução da rede para a representação do modelo de 'dois eixos'}

O sistema de potência a ser utilizado neste caso é o mesmo que foi apresentado nas seções anteriores salvo que os parâmetros da rede foram alterados (apenas por condição de estabilidade). A rede com os novos parâmetros é mostrado na figura 6.18 .

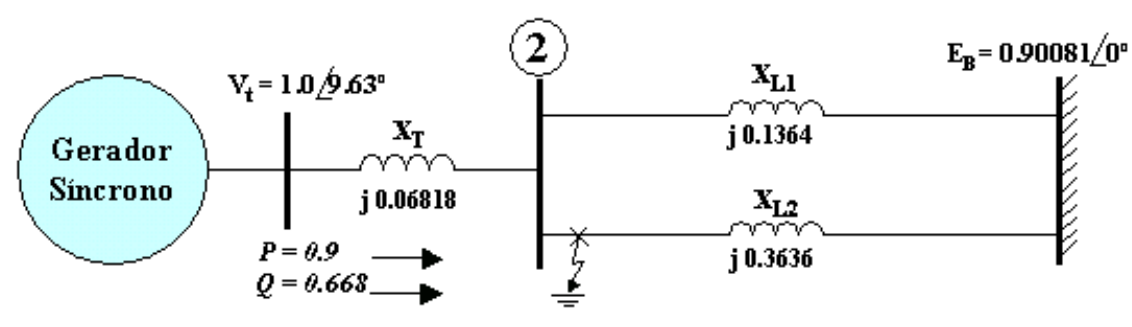

Figura 6.18: Sistema de potência para o a estimação de parâmetros do modelo de dois eixos

Para a solução das equações da rede, utiliza-se uma referência comum $R_{e}$ e $I_{m}$, onde o eixo $R_{e}$ coincide com o barramento infinito. Tal sistema de referência gira à velocidade síncrona juntamente com as tensões e correntes dos barramentos. As equações da máquina estão referidas aos eixos 'd 'e 'q' que giram com o rotor da máquina como é mostra a figura 6.19.

Para ter uma referência comum basta apenas projetar as componentes de uma referência para a outra. As tensões e correntes na referência $R_{e}$ e $I_{m}$ podem ser 


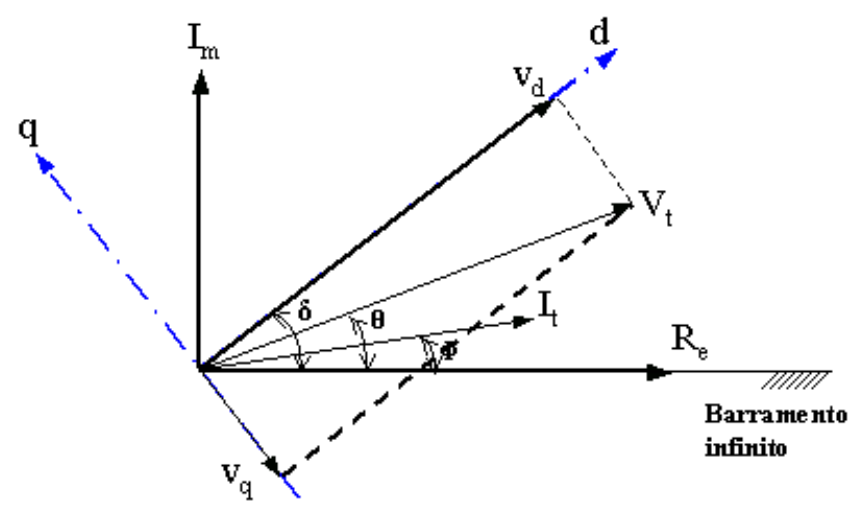

Figura 6.19: Sistema de referência da máquina e da rede externa

projetadas na referência 'd-q' através do vetor $e^{j \delta_{m}}$. Assim tem-se:

$$
\begin{aligned}
V_{t} & =\left(v_{q}+j v_{d}\right) e^{j \delta_{m}} \\
I_{t} & =\left(i_{q}+j i_{d}\right) e^{j \delta_{m}}
\end{aligned}
$$

Qualquer configuração da rede externa pode ser representada por dois parâmetros da rede em dois pontos. Nosso interesse é apenas o terminal da máquina síncrona, assim a tensão terminal é:

$$
V_{t}=\frac{I_{a}}{y_{11}}+h_{12} E_{b}
$$

Onde $y_{11}$ é a admitância de curto circuito da rede nos terminais do gerador, $h_{12}$ é o parâmetro híbrido (ganho de tensão em circuito aberto), e $E_{B}$ é a tensão no barramento infinito.

No caso de uma rede simples (máquina barramento infinito) com apenas uma impedância em série $\left(R_{e}+j x_{e}\right)$ tem-se:

$$
\begin{aligned}
& \frac{1}{y_{11}}=R_{e}+j x_{e} \\
& h_{12}=1+j 0
\end{aligned}
$$

A equação 6.13 pode ser expressa como:

$$
\left(v_{q}+j v_{d}\right) e^{j \delta}=\left(z_{R}+z_{I}\right)\left(i_{q}+j i_{d}\right) e^{j \delta}+\left(h_{1}+j h_{2}\right) E_{b}
$$

Multiplicando ambos os lados da equação por $e^{-j \delta}$, obtém-se:

$$
\left(v_{q}+j v_{d}\right)=\left(z_{R}+z_{I}\right)\left(i_{q}+j i_{d}\right)+\left(h_{1}+j h_{2}\right) E_{b} e^{-j \delta}
$$


Igualando as partes reais e as partes imaginárias, obtém-se:

$$
\begin{aligned}
& v_{q}=z_{R} i_{q}-Z_{I} i_{d}+h_{1} E_{b} \cos (\delta)+h_{2} E_{b} \sin (\delta) \\
& v_{d}=z_{I} i_{q}+Z_{R} i_{d}+h_{2} E_{b} \cos (\delta)-h_{1} E_{b} \sin (\delta)
\end{aligned}
$$

Assim, $I_{d}$ e $I_{q}$ são calculadas resolvendo-se o seguinte sistema de equações:

$$
\left(\begin{array}{cc}
\left(x_{d}^{\prime}+Z_{I}\right) & -\left(R_{a}+Z_{R}\right) \\
-\left(R_{a}+Z_{R}\right) & -\left(x_{q}^{\prime}+Z_{L}\right)
\end{array}\right)\left(\begin{array}{l}
i_{d} \\
i_{q}
\end{array}\right)=\left(\begin{array}{c}
-E_{q}^{\prime}+h_{1} E_{b} \cos (\delta)+h_{2} E_{b} \sin (\delta) \\
-E_{d}^{\prime}-h_{1} E_{b} \operatorname{sen}(\delta)+h_{2} E_{b} \cos (\delta)
\end{array}\right)
$$

As tensões no eixo direto e no eixo em quadratura são calculadas em função das variáveis de estado como :

$$
\begin{aligned}
& V_{q}=E_{q}^{\prime}+x_{d}^{\prime} i_{d}-R_{a} i_{q} \\
& V_{d}=E_{d}^{\prime}+x_{q}^{\prime} i_{q}+R_{a} i_{d}
\end{aligned}
$$

e a tensão terminal é dada por

$$
V_{t}=\sqrt{V_{d}^{2}+V_{q}^{2}}
$$

Os parâmetros do equivalente da rede em falta (figura 6.20 (a)) são: $Z_{R}=0$, $Z_{I}=0, h_{1}=0, h_{2}=0$

Os parâmetros do equivalente da rede em pós-falta (figura 6.20(b)) são: $Z_{R}=0$, $Z_{I}=X_{T}+X_{L I}, Z_{I}=0.2046 h_{1}=1, h_{2}=0$.

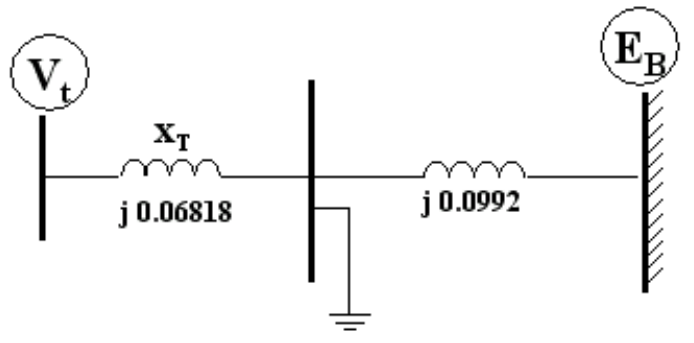

(a) Sistema em falta

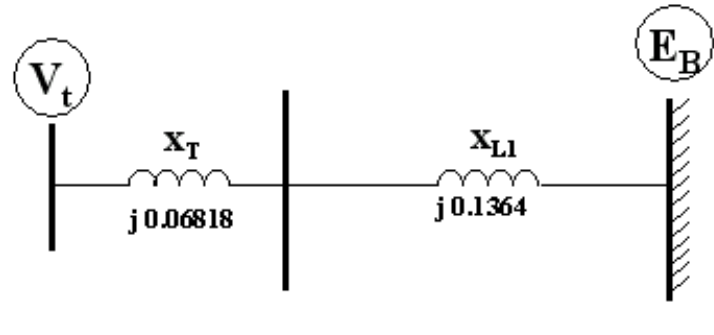

(b) Sistema em pós-falta

Figura 6.20: Sistema de potência reduzido nos terminais da máquina em falta e pós falta 


\section{Cálculo das condições iniciais}

Os valores iniciais para o sistema real são calculados como:

a)

$$
\begin{aligned}
I_{a} \angle \phi & =\frac{P-j Q}{V_{t} \angle-\theta} \\
I_{a} \angle \phi & =1.1208 \angle-26.95
\end{aligned}
$$

b)

$$
\begin{aligned}
& E_{q} O \angle \delta=V_{t} \angle \theta+\left(R_{a}+j x_{q}\right) I_{a} \\
& E_{q} O \angle \delta=2.6912 \angle 45.6864
\end{aligned}
$$

c)

$$
\begin{aligned}
& i_{d o}=I_{a} \operatorname{sen}(\delta-\phi) \\
& i_{d o}=-1.0698 \\
& i_{q o}=I_{a} \cos (\delta-\phi) \\
& i_{q o}=0.3344
\end{aligned}
$$

d)

$$
\begin{aligned}
& V_{d o}=V_{t} \operatorname{sen}(\delta-\phi) \\
& V_{d o}=-0.5886 \\
& V_{q o}=V_{t} \cos (\delta-\phi) \\
& V_{q o}=0.8084
\end{aligned}
$$

e)

$$
\begin{aligned}
& E_{f d o}=E_{q o}-\left(x_{d}-x_{q}\right) i_{d o} \\
& E_{f d o}=2.7447
\end{aligned}
$$

f)

$$
\begin{aligned}
& E_{q o}^{\prime}=E_{f d o}+\left(x_{d}-x_{d}^{\prime}\right) i_{d o} \\
& E_{q o}^{\prime}=1.1294
\end{aligned}
$$


g)

$$
\begin{aligned}
& E_{d o}^{\prime}=-\left(x_{q}-x_{q}^{\prime}\right) i_{q o} \\
& E_{d o}^{\prime}=-0.3712
\end{aligned}
$$

h)

$$
\begin{aligned}
& P_{e o}=E_{q o} i_{q o}+E_{d o}^{\prime} i_{d o}+\left(x_{d}^{\prime}-x_{q}^{\prime}\right) i_{d o} i_{q o} \\
& P_{e o}=0.9
\end{aligned}
$$

\section{Equações da máquina para o 'Sistema Real'}

O resumo das equações do modelo de dois eixos foi apresentado no capítulo 4 seção 5.1 .7 e é mostrado novamente aqui para facilitar a referência. A equação do sistema de excitação tipo ST1A está incluída nesta modelagem.

$$
\begin{aligned}
\dot{\delta} & =w \\
\dot{w} & =\frac{w_{o}}{2 H}\left[P_{m}-E_{q}^{\prime} I_{q}-E_{d}^{\prime} I_{d}-\left(x_{d}-x_{q}^{\prime}\right) I_{d} I_{q}-\frac{D}{w_{o}} w\right] \\
\dot{E}_{q}^{\prime} & =\frac{1}{T_{d o}^{\prime}}\left[E_{f d}-E_{q}^{\prime}+\left(x_{d}-x_{d}^{\prime}\right) I_{d}\right] \\
\dot{E_{d}^{\prime}} & =\frac{-1}{T_{q o}^{\prime}}\left[E_{d}^{\prime}+\left(x_{q}-x_{q}^{\prime}\right) I_{q}\right] \\
\dot{E_{f d}} & =\frac{1}{T_{R}}\left(K_{A}\left(V_{r e f}-V_{t}\right)-E_{f d}\right) \\
V_{q} & =E_{q}^{\prime}-r I_{q}+x_{d}^{\prime} I_{d} \\
V_{d} & =E_{d}^{\prime}-r I_{d}+x_{d}^{\prime} I_{q} \\
V_{t} & =\sqrt{V_{q}^{2}+V_{d}^{2}}
\end{aligned}
$$

As correntes do eixo direto e do eixo em quadratura podem ser obtidas resolvendo o sistema de equações (6.16). Para nosso estudo considera-se que a resistência do gerador e da linha de transmissão são desprezíveis. Assim uma simplificação das expressões algébricas das correntes em eixo direto e quadratura 
são calculadas como,

$$
\begin{aligned}
& I_{d}=\frac{E_{q}^{\prime}+h_{1} E_{b} \cos (\delta)}{x_{d}^{\prime}+Z_{I}} \\
& I_{q}=\frac{E_{q}^{\prime}+h_{1} E_{b} \operatorname{sen}(\delta)}{x_{q}^{\prime}+Z_{I}}
\end{aligned}
$$

onde $h_{1}$ é um fator que depende das condições de funcionamento, sendo igual a zero em regime de falta e igual a um em pós falta (segundo análise realizada das condições do sistema de potência anteriormente). A seguir são apresenta-se uma seqüência de testes para a identificação de parâmetros.

\subsubsection{Teste $\mathrm{N}^{\circ}$ 1: Estimação de parâmetros da máquina síncrona considerando como entradas $I_{d}$ e $I_{q}$ e $E_{f d}$, e como saida $w$}

Neste primeiro teste $I_{d}, I_{q}$, e $E_{f d}$ são as variáveis de entrada, e $w$ é a variável de saída. Fazendo isto as equações para o modelo matemático são definidas como:

$$
\begin{aligned}
\dot{w_{m}} & =\frac{\omega_{o}}{2 H}\left[P_{m}-E_{q m}^{\prime} I_{q}-E_{d m}^{\prime} I_{d}-\left(x_{d}-x_{q}^{\prime}\right) I_{d} I_{q}-\frac{D}{\omega_{o}} \omega_{m}\right] \\
E_{q m}^{i} & =\frac{1}{T_{d o}^{\prime}}\left[E_{f d}-E_{q m}^{\prime}+\left(x_{d}-x_{d}^{\prime}\right) I_{d}\right] \\
E_{d m}^{i} & =\frac{-1}{T_{q o}^{\prime}}\left[E_{d m}^{\prime}+\left(x_{q}-x_{q}^{\prime}\right) I_{q}\right]
\end{aligned}
$$

Onde o sub-índice 'm' denota variável do modelo matemático.

Nota-se que com as variáveis de entrada escolhidas as equações diferenciais relacionadas a $(\delta)$ e a $\left(E_{f d}\right)$ não são consideradas na modelagem do modelo matemático.

Os parâmetros a serem estimados estão incluídos no vetor de parâmetros 'p' sendo:

$$
p=\left[\begin{array}{llllll}
x_{d} & x_{d}^{\prime} & T_{d o}^{\prime} & x_{q} & x_{q}^{\prime} & T_{q o}^{\prime}
\end{array}\right]^{t}
$$

As funções de sensibilidade são ser encontradas derivando-se as equações diferenciais do modelo matemático e integrando-as no tempo. Para facilitar a análise,

substitui-se $\lambda_{f}^{p}$ por $\frac{\partial f}{\partial p}$ nas derivadas parciais do modelo matemático e obtém-se: 
Derivando em relação a $x_{d}$

$$
\left(\begin{array}{l}
\dot{\lambda}_{w_{m}}^{x_{d}} \\
\dot{\lambda}_{E_{q m}^{\prime}}^{x_{d}} \\
\dot{\lambda}_{E_{d m}^{\prime}}^{x_{d}}
\end{array}\right)=\left(\begin{array}{c}
\frac{\omega_{o}}{2 H}\left(-\lambda_{E_{q m}^{\prime}}^{x_{d}} I_{q}-\lambda_{E_{d m}^{\prime}}^{x_{d}} I_{d}-\frac{D}{\omega_{o}} \lambda_{w_{m}}^{x_{d}}\right) \\
\frac{1}{T_{d o}^{\prime}}\left(-\lambda_{E_{q m}^{\prime}}^{x_{d}}+I_{d}\right) \\
-\frac{1}{T_{q o}^{\prime}}\left(\lambda_{E_{d}^{\prime} m}^{x_{d}}\right)=0
\end{array}\right)
$$

Derivando em relação a $x_{d}^{\prime}$

$$
\left(\begin{array}{l}
\dot{\lambda}_{w_{m}^{\prime}}^{x_{d m}^{\prime}} \\
\dot{\lambda}_{E_{d m}^{\prime}}^{x_{d}^{\prime}} \\
\dot{\lambda}_{E_{d m}^{\prime}}^{x_{d}^{\prime}}
\end{array}\right)=\left(\begin{array}{c}
\frac{\omega_{o}}{2 H}\left(-\lambda_{E_{q m}^{\prime}}^{x_{d}^{\prime}} I_{q}-I_{d} I_{q}-\lambda_{E_{d m}^{\prime}}^{x_{d}^{\prime}} I_{d}-\frac{D}{\omega_{o}} \lambda_{w_{m}}^{x_{d}^{\prime}}\right) \\
\frac{1}{T_{d o}^{\prime}}\left(-\lambda_{E_{q m}^{\prime}}^{x^{\prime}}-I_{d}\right) \\
-\frac{1}{T_{q o}^{\prime}}\left(\lambda_{E_{d m}^{\prime}}^{x_{d}^{\prime}}\right)=0
\end{array}\right)
$$

Derivando em relação a $x_{q}$

$$
\left(\begin{array}{c}
\dot{\lambda}_{w_{m}}^{x_{q}} \\
\dot{\lambda}_{E_{q}^{\prime}}^{x_{q}} \\
\dot{\lambda}_{E_{d m}^{\prime}}^{x_{q}}
\end{array}\right)=\left(\begin{array}{c}
\frac{\omega_{o}}{2 H}\left(-\lambda_{E_{q m}^{\prime}}^{x_{q}} I_{q}-\lambda_{E_{d m}^{\prime}}^{x_{q}} I_{d}-\frac{D}{\omega_{o}} \lambda_{w_{m}}^{x_{q}}\right) \\
\frac{1}{T_{d o}^{\prime}}\left(-\lambda_{E_{q m}^{\prime}}^{x_{q}}\right)=0 \\
-\frac{1}{T_{q o}^{\prime}}\left(\lambda_{E_{d m}^{\prime}}^{x_{q}}+I_{q}\right)=0
\end{array}\right)
$$

Derivando em relação a $x_{q}^{\prime}$

$$
\left(\begin{array}{l}
\dot{\lambda}_{w_{m}}^{x_{q}^{\prime}} \\
\dot{\lambda}_{E_{q m}^{\prime}}^{x^{\prime}} \\
\dot{\lambda}_{E_{d m}^{\prime}}^{x_{q}^{\prime}}
\end{array}\right)=\left(\begin{array}{c}
\frac{\omega_{o}}{2 H}\left(-\lambda_{E_{q m}^{\prime}}^{x_{q}^{\prime}} I_{q}+I_{d} I_{q}-\lambda_{E_{d m}^{\prime}}^{x_{d}^{\prime}} I_{d}-\frac{D}{\omega_{o}} \lambda_{w_{m}}^{x_{q}^{\prime}}\right) \\
\frac{1}{T_{d o}^{\prime}}\left(-\lambda_{E_{q m}^{\prime}}^{x_{q}}\right)=0 \\
-\frac{1}{T_{q o}^{\prime}}\left(\lambda_{E_{d m}^{\prime}}^{x_{q}^{\prime}}-I_{q}\right)
\end{array}\right)
$$

Derivando em relação a $T_{d o}^{\prime}$

$$
\left(\begin{array}{l}
\dot{\lambda}_{w_{m}}^{T_{d o}^{\prime}} \\
\dot{\lambda}_{E_{d o}^{\prime}}^{T_{d o}^{\prime}} \\
\dot{\lambda}_{E_{d m}^{\prime}}^{T_{d o}^{\prime}}
\end{array}\right)=\left(\begin{array}{c}
\frac{\omega_{o}}{2 H}\left(-\lambda_{E_{q m}^{\prime}}^{T_{d o}^{\prime}} I_{q}-\lambda_{E_{d m}^{\prime}}^{T_{d o}^{\prime}} I_{d}-\frac{D}{\omega_{o}} \lambda_{w_{m}}^{T_{d o}^{\prime}}\right) \\
-\frac{1}{T_{d o}^{\prime 2}}\left(E_{f d}-E_{q m}^{\prime}+\left(x_{d}-x_{d}^{\prime}\right) I d\right)+\frac{1}{T_{d o}^{\prime}}\left(-\lambda_{E_{q m}^{\prime}}^{T_{d o}^{\prime}}\right) \\
-\frac{1}{T_{q o}^{\prime}}\left(\lambda_{E_{d m}^{\prime}}^{T_{d o}^{\prime}}\right)=0
\end{array}\right)
$$

Derivando em relação a $T_{q o}^{\prime}$

$$
\left(\begin{array}{l}
\dot{\lambda}_{w_{m}}^{T_{q o}^{\prime}} \\
\dot{\lambda}_{E_{q m}^{\prime}}^{T_{q}^{\prime}} \\
\dot{\lambda}_{E_{d m}^{\prime}}^{T_{q}^{\prime}}
\end{array}\right)=\left(\begin{array}{c}
\frac{\omega_{o}}{2 H}\left(-\lambda_{E_{q m}^{\prime}}^{T_{q o}^{\prime}} I_{q}-\lambda_{E_{d m}^{\prime}}^{T_{q o}^{\prime}} I_{d}-\frac{D}{\omega_{o}} \lambda_{w_{m}}^{T_{q o}^{\prime}}\right) \\
-\frac{1}{T_{d o}^{\prime}}\left(-\lambda_{E_{q m}^{\prime}}^{T_{q o}^{\prime}}\right)=0 \\
\frac{1}{T_{q o}^{\prime 2}}\left(E_{d m}^{\prime}+\left(x_{q}-x_{q}^{\prime}\right) I_{q}\right)-\frac{1}{T_{q o}^{\prime}}\left(\lambda_{E_{d m}^{\prime}}^{T_{q o}^{\prime}}\right)
\end{array}\right)
$$

As condições iniciais das derivadas parciais anteriores são iguais a zero, pois tais pontos correspondem às derivadas das condições iniciais do modelo matemático em relação aos parâmetros (equação 3.11). 
Para aplicar o processo de estimação precisamos das condições iniciais das equações diferenciais do modelo matemático $\omega\left(t_{o}\right), E_{d}^{\prime}\left(t_{o}\right)$ e $E_{q}^{\prime}\left(t_{o}\right)$. Entretanto o cálculo das condições iniciais de $E_{d}^{\prime}$ e $E_{q}^{\prime}$ estão em função dos parâmetros que queremos identificar. Obter tais valores diretamente do sistema real é difícil pois estas variáveis estão relacionadas aos fluxos no eixo direto e em quadratura.

Uma alternativa para contornar este problema é considerar $E_{d}^{\prime}\left(t_{o}\right)$ e $E_{q}^{\prime}\left(t_{o}\right)$ como parâmetros a serem estimados conjuntamente com os outros parâmetros da máquina, bastando para isto apenas estimativas iniciais. Para simplificar as equações denota-se $E_{q m}^{\prime}\left(t_{o}\right)$ por $E_{q o}^{\prime}$ e $E_{d m}^{\prime}\left(t_{o}\right)$ por $E_{d o}^{\prime}$ na seqüência do texto. O vetor de parâmetros a estimar é:

$$
p=\left[\begin{array}{llllllll}
x_{d} & x_{d}^{\prime} & T_{d o}^{\prime} & x_{q} & x_{q}^{\prime} & T_{q o}^{\prime} & E_{q o}^{\prime} & E_{d o}^{\prime}
\end{array}\right]^{t}
$$

As funções de sensibilidade em relação aos novos parâmetros são obtidas derivando-se as equações diferenciais do modelo matemático em relação a $E_{q o}^{\prime}$ e $E_{d o}^{\prime}$ e integrando-as no tempo.

As derivadas parciais das equações do modelo matemático são mostradas a seguir:

Derivando as equações do modelo matemático em relação a $E_{q o}^{\prime}$

$$
\left(\begin{array}{l}
\dot{\lambda}_{w_{m}}^{E_{q o}^{\prime}} \\
\dot{\lambda}_{E_{q o}^{\prime}}^{E_{q}^{\prime}} \\
\dot{\lambda}_{E_{d m}^{\prime}}^{E_{d o}^{\prime}}
\end{array}\right)=\left(\begin{array}{c}
\frac{\omega_{o}}{2 H}\left(-\lambda_{E_{q m}^{\prime}}^{E_{o}^{\prime}} I_{q}-\lambda_{E_{d o}^{\prime}}^{E_{d}^{\prime}} I_{d}-\frac{D}{\omega_{o}} \lambda_{w_{m}}^{E_{q o}^{\prime}}\right) \\
\frac{1}{T_{d o}^{\prime}}\left(-\lambda_{E_{q m}^{\prime}}^{E^{\prime}}\right) \\
-\frac{1}{T_{q o}^{\prime}}\left(\lambda_{E_{d m}^{\prime}}^{E^{\prime}}\right)
\end{array}\right)
$$

As condições iniciais são:

$$
\lambda_{w_{m}}^{E_{q o}^{\prime}}\left(t_{0}\right)=0 \quad \lambda_{E_{q m}^{\prime}}^{E_{q o}^{\prime}}\left(t_{0}\right)=1 \quad \lambda_{E_{d m}^{\prime}}^{E_{q o}^{\prime}}\left(t_{0}\right)=0
$$

Derivando as equações do modelo matemático em relação a $E_{d o}^{\prime}$

$$
\left(\begin{array}{l}
\dot{\lambda}_{w_{m}}^{E_{d o}^{\prime}} \\
\dot{\lambda}_{E_{d m}^{\prime}}^{E_{d o}^{\prime}} \\
\dot{\lambda}_{E_{d m}^{\prime}}^{E_{d o}^{\prime}}
\end{array}\right)=\left(\begin{array}{c}
\frac{\omega_{o}}{2 H}\left(-\lambda_{E_{q m}^{\prime}}^{E_{d o}^{\prime}} I_{q}-\lambda_{E_{d m}^{\prime}}^{E_{d o}^{\prime}} I_{d}-\frac{D}{\omega_{o}} \lambda_{w_{m}}^{E_{d o}^{\prime}}\right) \\
\frac{1}{T_{d o}^{\prime}}\left(-\lambda_{E_{q m}^{\prime}}^{E_{d o}^{\prime}}\right)=0 \\
-\frac{1}{T_{q o}^{\prime}}\left(\lambda_{E_{d m}^{\prime}}^{E_{d o}^{\prime}}\right)
\end{array}\right)
$$

As condições iniciais são:

$$
\lambda_{w_{m}}^{E_{d o}^{\prime}}\left(t_{0}\right)=0 \quad \lambda_{E_{q m}^{\prime}}^{E_{d o}^{\prime}}\left(t_{0}\right)=0 \quad \lambda_{E_{d m}^{\prime}}^{E_{d o}^{\prime}}\left(t_{0}\right)=1
$$


As condições iniciais $\lambda_{E_{q m}^{\prime}}^{E_{q o}^{\prime}}\left(t_{0}\right)$ e $\lambda_{E_{d m}^{\prime}}^{E_{d o}^{\prime}}\left(t_{0}\right)$, são diferentes de zero, pois no ponto inicial a variação de $E_{q m}^{\prime}$ e $E_{d m}^{\prime}$ em relação a $E_{q o}^{\prime}$ e $E_{d o}^{\prime}$ é igual a 1 . Isto pode ser analisado na figura 6.21 .

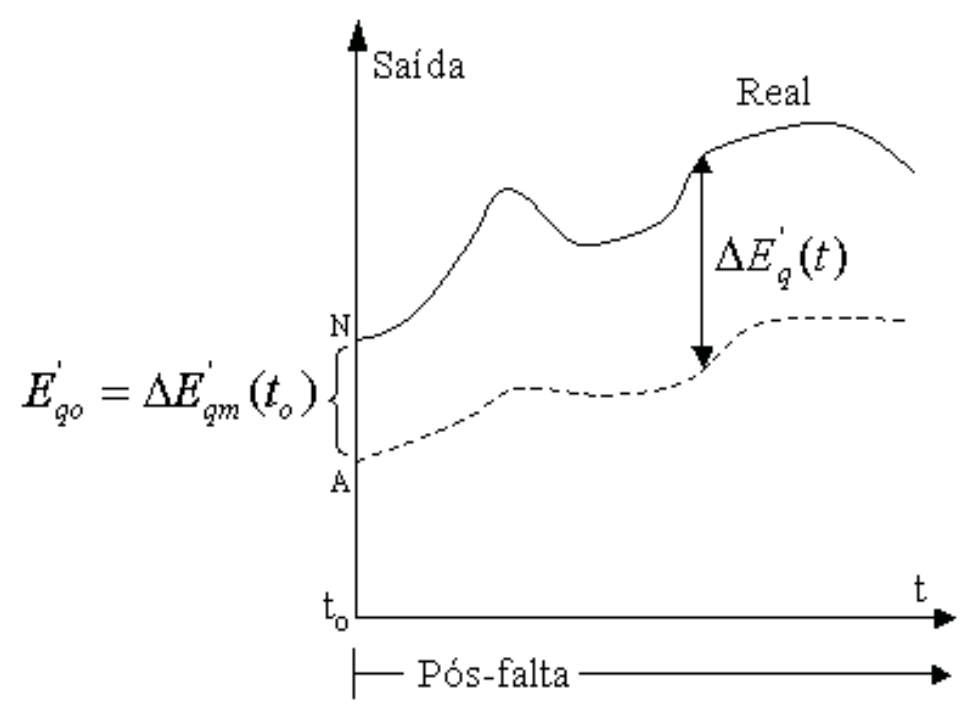

Figura 6.21: Determinação da condição inicial para o calculo da função de sensibilidade em relação a $E_{d o}^{\prime}$

No ponto inicial $t_{o}$ temos,

$$
\begin{aligned}
\frac{\partial E_{q m}}{\partial E_{q o}}\left(t_{o}\right) & \cong \frac{\Delta E_{q m}}{\Delta E_{q o}}\left(t_{o}\right) \\
\frac{\triangle E_{q m}}{\triangle E_{q o}}\left(t_{o}\right) & =\frac{E_{q m}(N)-E_{q m}(A)}{\Delta E_{q o}}=\frac{\Delta E_{q o}}{\Delta E_{q o}} \\
\frac{\partial E_{q m}}{\partial E_{q o}}\left(t_{o}\right) & =1
\end{aligned}
$$

O diagrama esquemático de entradas e saídas do 'modelo matemático' e do 'sistema real' é mostrado na figura 6.22 .

Na seqüência, a metodologia é aplicada para a estimação dos parâmetros primeiro do eixo direto e depois de eixo em quadratura e finalmente para estimação dos 6 parâmetros elétricos da máquina síncrona simultaneamente.

\section{Estimação dos parâmetros de eixo direto}

Neste teste são estimados os parâmetros de eixo direto.

$$
p=\left[\begin{array}{lll}
x_{d} & x_{d}^{\prime} & T_{d o}^{\prime}
\end{array}\right]^{t}
$$




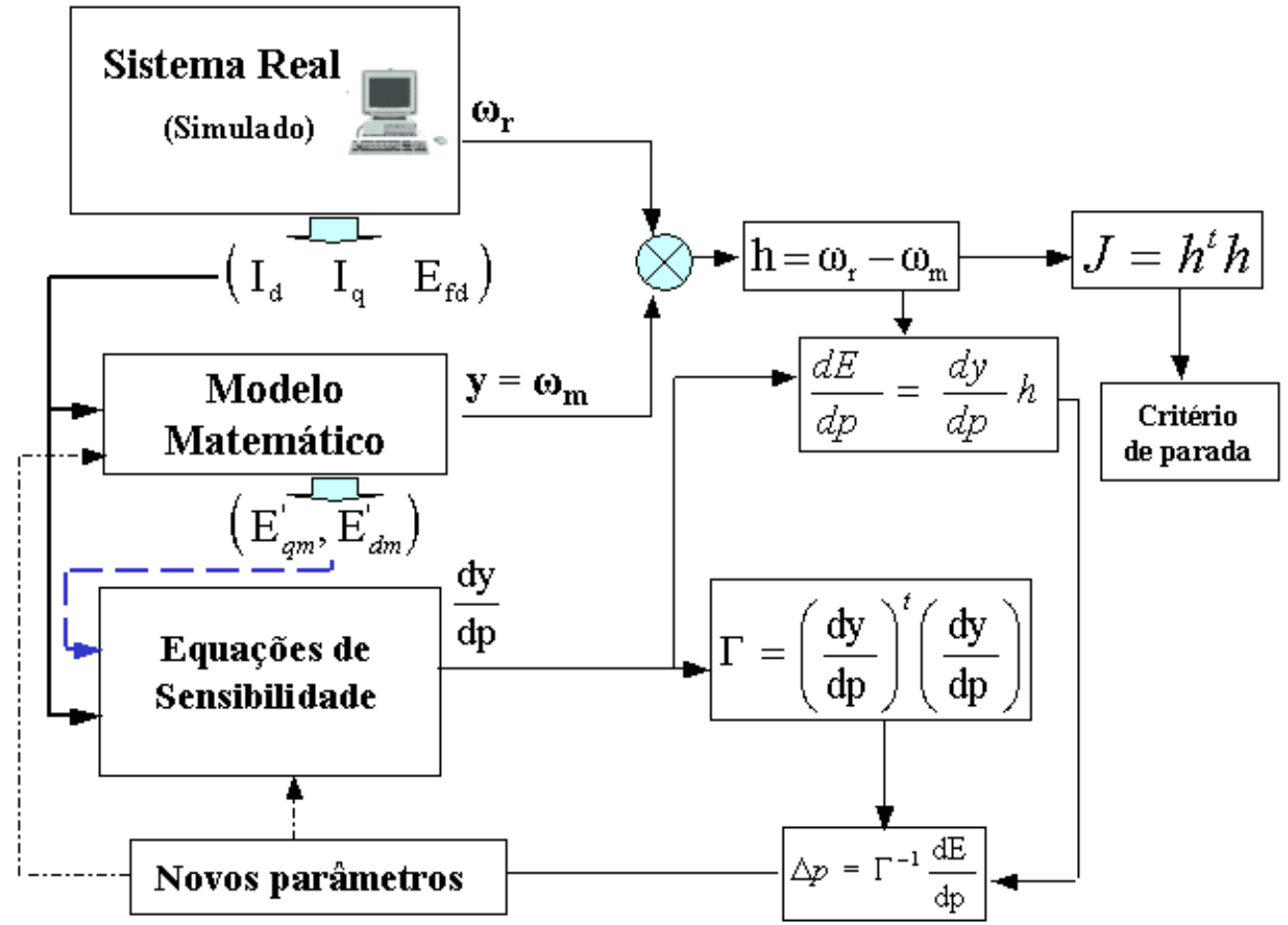

Figura 6.22: Diagrama esquemático para o teste 1 com $I_{d}$ e $I_{q}$ como entradas

Inicialmente, considera-se que os parâmetros iniciais do 'modelo matemático' estão distantes em -20\% dos valores verdadeiros. Aplicando a metodologia com a condição de perturbação descrita anteriormente os parâmetros foram estimados com sucesso.

Na figura 6.23 mostra-se a saída do sistema real e do modelo matemático antes e depois da estimação de parâmetros. Os parâmetros foram estimados após quatro iterações.

Na tabela 6.4 mostra-se o resumo do processo de estimação dos parâmetros de eixo direto.

Tabela 6.4: Estimação dos parâmetros de eixo direto no teste $\mathrm{n}^{\circ} 1$

\begin{tabular}{|c|c|c|c|c|}
\hline parâmetro & V. Inicial & V. final & V. Real & $\operatorname{Erro}(\%)$ \\
\hline$x_{d}$ & 1.4480 & 1.81 & 1.81 & $0(\%)$ \\
\hline$x_{d}^{\prime}$ & 0.2400 & 0.30 & 0.30 & $0(\%)$ \\
\hline$T_{d o}^{\prime}$ & 6.400 & 8.0 & 7.9988 & $-0.015(\%)$ \\
\hline
\end{tabular}



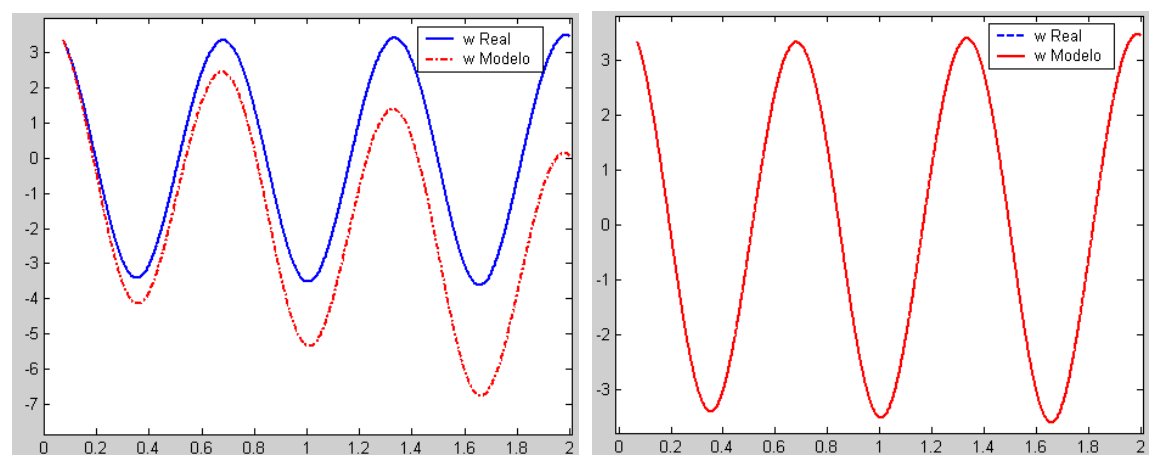

Figura 6.23: Velocidade angular antes e depois da estimação dos parâmetros do eixo d no teste $n^{\circ} 1$

\section{Estimação dos parâmetros de eixo em quadratura}

Neste teste são estimados os parâmetros do eixo em quadratura.

$$
p=\left[\begin{array}{lll}
x_{q} & x_{q}^{\prime} & T_{q o}^{\prime}
\end{array}\right]^{t}
$$

Inicialmente, considera-se que os parâmetros inicias do 'modelo matemático' estão distantes em $-30 \%$ dos valores verdadeiros. Na figura 6.24 mostra-se a saída do 'sistema real' e do 'modelo matemático' antes e depois a estimação de parâmetros. Os parâmetros foram estimados depois de quatro iterações.
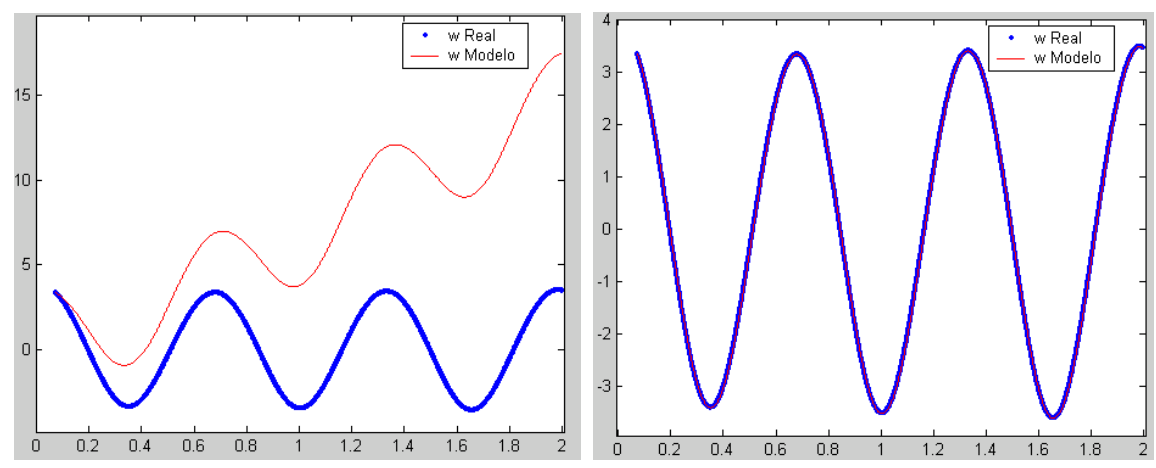

Figura 6.24: Velocidade angular w antes e depois da estimação dos parâmetros do eixo em quadratura no teste $n^{\circ} 1$

O resumo dos resultados é mostrado na tabela 6.5. Os parâmetros foram estimados com um erro inferior a $0.02 \%$ 


\begin{tabular}{|c|c|c|c|c|}
\hline parâmetro & V. Inicial & V. final & V. Real & Erro(\%) \\
\hline$x_{d}$ & 1.4480 & 1.81 & 1.81 & $0(\%)$ \\
\hline$x_{d}^{\prime}$ & 0.2400 & 0.30 & 0.30 & $0(\%)$ \\
\hline$T_{d o}^{\prime}$ & 6.400 & 7.9988 & 8.0 & $-0.015(\%)$ \\
\hline
\end{tabular}

Tabela 6.5: Estimação dos parâmetros do eixo em quadratura no teste $\mathrm{n}^{\circ} 1$

\section{Estimação simultânea dos parâmetros de eixo direto e quadratura}

Neste caso a metodologia aplicada para estimar todos os parâmetros elétricos da máquina síncrona em forma simultânea. Entretanto os resultados não foram satisfatórios, pois a matriz gama $(\Gamma)$ se torna singular e o procedimento diverge. Problemas similares foram relatados em (Sanchez et al., 1988) relacionados com a estimação simultânea dos parâmetros de eixo direto e de eixo em quadratura.

Para entender-se o problema da não convergência do processo de estimação realiza-se uma análise da primeira iteração. Neste teste o vetor dos parâmetros a serem determinados é:

$$
p=\left[\begin{array}{llllll}
x_{d} & x_{d}^{\prime} & T_{d o}^{\prime} & x_{q} & x_{q}^{\prime} & T_{q o}^{\prime}
\end{array}\right]^{t}
$$

As funções de sensibilidade calculadas na primeira iteração são mostradas na figura 6.25 .
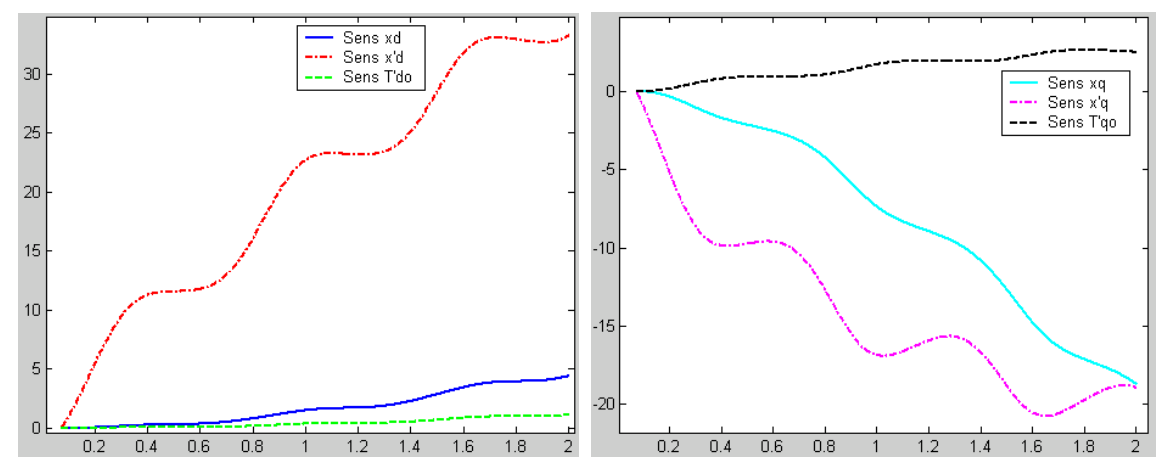

Figura 6.25: Funções de sensibilidade da saída 'w' em relação aos parâmetros para a primeira iteração.

Todas as funções de sensibilidade são diferentes de zero e, em principio, não é possível tirar alguma informação que permita entender o problema. Calcula-se 
então a matriz $\Gamma$ para a primeira iteração,

$$
\Gamma=1 * 10^{3}\left(\begin{array}{cccccc}
0.0067 & 0.0786 & 0.0011 & -0.0358 & -0.0498 & 0.0060 \\
0.0786 & 1.0032 & 0.0132 & -0.4258 & -0.6560 & 0.0760 \\
0.0011 & 0.0132 & 0.0002 & -0.0060 & -0.0083 & 0.0010 \\
-0.0358 & -0.4258 & -0.006 & 0.1911 & 0.2705 & -0.0325 \\
-0.0496 & -0.6560 & -0.0083 & 0.2705 & 0.4351 & -0.0496 \\
0.0060 & 0.0760 & 0.0010 & -0.0325 & -0.0496 & 0.0058
\end{array}\right)
$$

O posto desta matriz é cinco, isto indica que existe uma coluna linearmente dependente das outras. Uma alternativa para contornar esta dificuldade é eliminar a coluna da matriz de sensibilidade que apresenta dependência linear. O ajuste do parâmetro relacionado com a função de sensibilidade dependente deve ser realizado através de uma equação algébrica a determinar.

Fatorando a matriz $\Gamma$ aparece um pivô zero no elemento $(5,5)$, portanto, a coluna $C_{5}$ é linearmente dependente das outras. Para encontrar a dependência linear desta coluna elimina-se a linha $L_{5}$ da matriz gama e faz-se:

$$
C_{5}=\alpha_{1} C_{1}+\alpha_{2} C_{2}+\alpha_{3} C_{3}+\alpha_{4} C_{4}+\alpha_{6} C_{6}
$$

Resolvendo o sistema anterior obtém-se:

$$
\alpha_{1}=-1 \quad \alpha_{2}=-1 \quad \alpha_{3}=0 \quad \alpha_{4}=-1 \quad \alpha_{6}=0
$$

Assim a quinta coluna tem dependência linear da primeira, a segunda e a quarta coluna. Por outro lado as colunas da matriz gama estão relacionadas com as funções de sensibilidades da saída em relação aos parâmetros. A primeira coluna corresponde a sensibilidade em relação a $x_{d}$, a segunda corresponde a $x_{d}^{\prime}$, a quarta a $x_{q}$ e a quinta a $x_{q}^{\prime}$.

A análise anterior indica que mantendo-se fixo algum dos parâmetros do conjunto $\left(x_{d}, x_{d}^{\prime}, x_{q}, x_{q}^{\prime}\right)$ a matriz $\Gamma$ deixa de ser singular e o procedimento de estimação fica bem definido. Este fato foi comprovado por intermédio dos testes.

No teste a seguir mostra-se que, mantendo fixo o parâmetro $x_{d}^{\prime}$ os outros parâmetros podem ser estimados satisfatoriamente. Neste teste $E_{d o}^{\prime}$ e $E_{q o}^{\prime}$ foram 
considerados como parâmetros e estimados simultaneamente pela metodologia. Na figura 6.26 mostra-se a saída do 'modelo matemático' e do 'sistema real' antes e depois da estimação dos parâmetros, mantendo o parâmetro $x_{d}$ fixo. Os valores iniciais utilizados no modelo matemático correspondem aos valores verdadeiros alterados em $-30 \%$ (para os parâmetros de eixo direto),em $+30 \%$ (para os parâmetros de eixo em quadratura) e $+30 \%$ para $E_{d o}^{\prime}$ e $E_{q o}^{\prime}$.
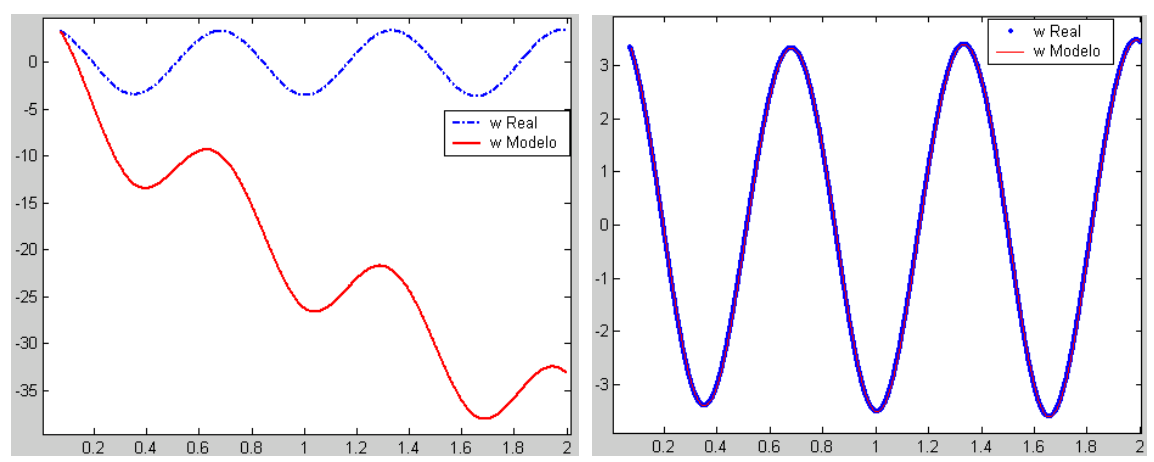

Figura 6.26: Velocidade angular antes e depois da estimação dos parâmetros com $x_{d}^{\prime}$ fixo

Na tabela 6.6 são apresentados os resultados obtidos depois da aplicação da metodologia.

Tabela 6.6: Estimação de parâmetros da máquina síncrona com mantendo $x_{d}^{\prime}=0.3$ no teste $\mathrm{n}^{\circ} 1$

\begin{tabular}{|c|c|c|c|c|}
\hline parâmetro & V. Inicial & V. final & V. Real & Erro(\%) \\
\hline$x_{d}$ & 1.2670 & 1.8100 & 1.8100 & $0(\% 0.0)$ \\
\hline$T_{d o}^{\prime}$ & 5.6000 & 7.9992 & 8.0000 & $-0.01(\%)$ \\
\hline$x_{q}$ & 2.2880 & 1.7600 & 1.7600 & $0.0(\%)$ \\
\hline$x_{q}^{\prime}$ & 0.8450 & 0.6500 & 0.6500 & $0.0(\%)$ \\
\hline$T_{q o}^{\prime}$ & 1.3000 & 1.000 & 1.000 & $0.0(\%)$ \\
\hline$E_{q o}^{\prime}$ & 1.7493 & 1.1662 & 1.1662 & $0.0(\%)$ \\
\hline$E_{d o}^{\prime}$ & -0.4666 & -0.3110 & -0.3110 & $0.0(\%)$ \\
\hline
\end{tabular}




\subsubsection{Teste $\mathrm{N}^{\circ}$ 2: Estimação de parâmetros da máquina síncrona considerando $E_{f d}$ como entrada e $w$ como saída}

Neste teste apenas $E_{f d}$ é escolhida como entrada e $w$ é escolhida como saída. Fazendo isto as equações diferenciais para o modelo matemático são dados por:

$$
\begin{aligned}
\dot{\delta_{m}} & =\omega_{m} \\
\dot{w_{m}} & =\frac{\omega_{o}}{2 H}\left[P_{m}-E_{q m}^{\prime} I_{q}-E_{d m}^{\prime} I_{d}-\left(x_{d}-x_{q}^{\prime}\right) I_{d} I_{q}-\frac{D}{\omega_{o}} \omega_{m}\right] \\
\dot{E_{q m}^{i}} & =\frac{1}{T_{d o}^{\prime}}\left[E_{f d}-E_{q m}^{\prime}+\left(x_{d}-x_{d}^{\prime}\right) I_{d}\right] \\
E_{d m}^{i} & =\frac{-1}{T_{q o}^{\prime}}\left[E_{d m}^{\prime}+\left(x_{q}-x_{q}^{\prime}\right) I_{q}\right]
\end{aligned}
$$

Neste caso, pela entrada e saída escolhida, que a dinâmica correspondente a $\delta$ é considerada. Utiliza-se sub-índice 'm' para denotar as variáveis do modelo matemático.

As correntes $I_{d}$ e $I_{q}$ neste caso são calculadas em cada iteração através das seguintes equações:

$$
\begin{aligned}
& I_{d}=\frac{E_{b} \cos \left(\delta_{m}\right)-E_{q m}^{\prime}}{x_{d}^{\prime}+Z_{I}} \\
& I_{q}=\frac{E_{b} \operatorname{sen}\left(\delta_{m}\right)+E_{d m}^{\prime}}{x_{q}^{\prime}+Z_{I}}
\end{aligned}
$$

sendo $E_{b}$ a tensão no barramento infinito e $Z_{I}$ a impedância equivalente em pósfalta.

O diagrama esquemático mostrando as entradas e saídas do 'modelo matemático' e do 'modelo de sensibilidade' é apresentado na figura 6.27. O vetor dos parâmetros a serem determinado vem dado por:

$$
p=\left[\begin{array}{llllllll}
x_{d} & x_{d}^{\prime} & T_{d o}^{\prime} & x_{q} & x_{q}^{\prime} & T_{q o}^{\prime} & E_{q o}^{\prime} & E_{d o}^{\prime}
\end{array}\right]^{t}
$$

Os valores dos parâmetros verdadeiros foram alterados em $+30 \%$ (incluindo $E_{q o}^{\prime}$ e $\left.E_{d o}^{\prime}\right)$ e utilizados como valores iniciais para o 'modelo matemático'. Aplicando 


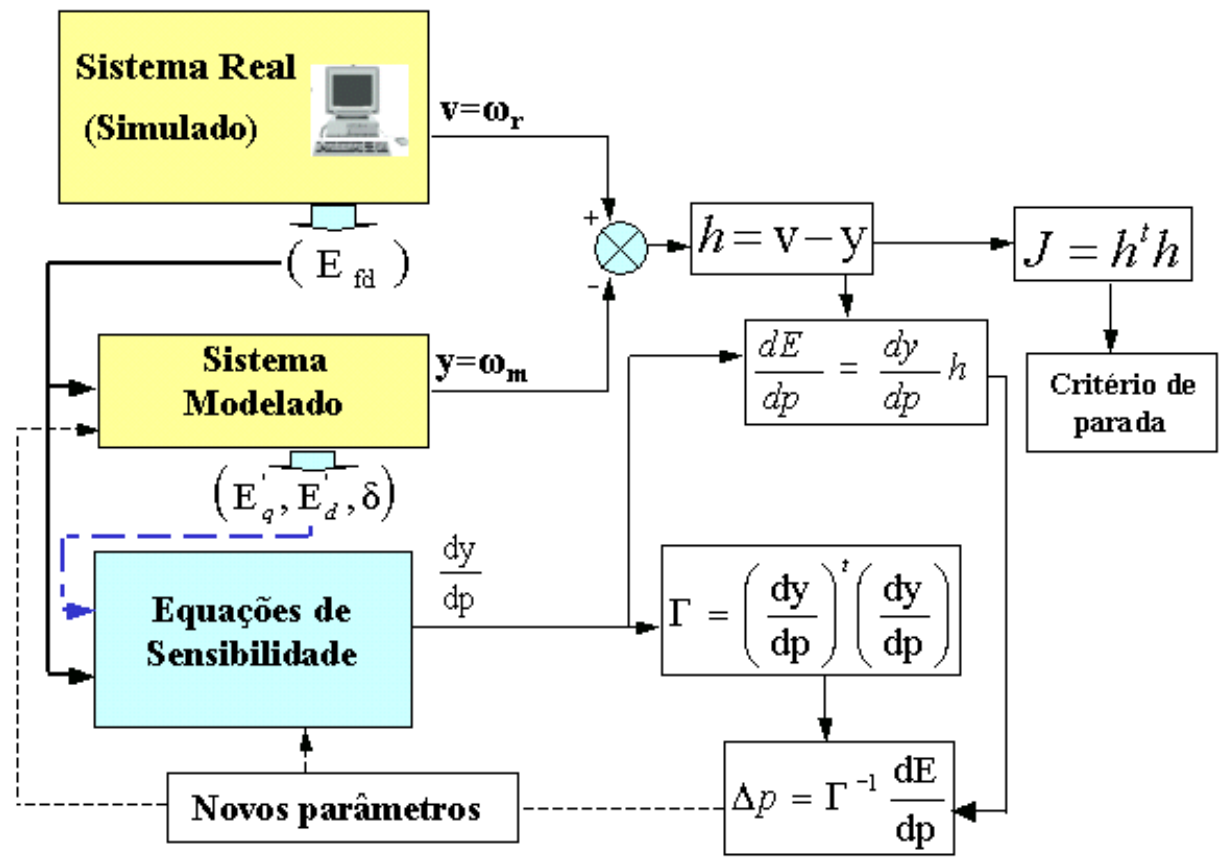

Figura 6.27: Diagrama esquemático de entradas e saídas no teste $\mathrm{n}^{\circ} 2$

a metodologia, os parâmetros convergiram para os valores verdadeiros em 9 iterações. Na tabela 6.7 mostra-se o resumo dos resultados do processo de estimação. Nesta modelagem não é necessário fixar nenhum dos parâmetros.

Tabela 6.7: Estimação dos parâmetros da máquina síncrona no teste $\mathrm{n}^{\circ} 2$, incluindo $E_{d o}^{\prime}$ e $E_{q o}^{\prime}$.

\begin{tabular}{|c|c|c|c|c|}
\hline parâmetro & V. Inicial & V. final & V. Real & Erro(\%) \\
\hline$x_{d}$ & 2.3530 & 1.8102 & 1.8100 & 0.011 \\
\hline$x_{d}^{\prime}$ & 0.3900 & 0.300 & 0.300 & 0.0 \\
\hline$T_{d o}^{\prime}$ & 10.400 & 8.0003 & 8.0000 & 0.03 \\
\hline$x_{q}$ & 2.2880 & 1.7587 & 1.7600 & -0.07 \\
\hline$x_{q}^{\prime}$ & 0.8450 & 0.6499 & 0.6500 & -0.015 \\
\hline$T_{q o}^{\prime}$ & 1.3000 & 0.9997 & 1.000 & -0.03 \\
\hline$E_{q o}^{\prime}$ & 1.5161 & 1.1661 & 1.1662 & -0.008 \\
\hline$E_{d o}^{\prime}$ & -0.4044 & -0.3110 & -0.3110 & -0.0003 \\
\hline
\end{tabular}

As estimativas iniciais para $E_{q o}^{\prime}$ e $E_{d o}^{\prime}$ podem ser calculadas através das seguintes 
equações:

$$
\begin{aligned}
V_{d o} & =-V_{t o} \operatorname{sen}\left(\delta_{o}-\theta_{o}\right) \\
V_{q o} & =-V_{t o} \cos \left(\delta_{o}-\theta_{o}\right) \\
I_{d o} & =-I_{t o} \operatorname{sen}\left(\delta_{o}-\phi_{o}\right) \\
I_{q o} & =I_{t o} \cos \left(\delta_{o}-\phi_{o}\right) \\
E_{d o}^{\prime} & =V_{q o}-x_{d}^{\prime} I_{d o} \\
E_{q o}^{\prime} & =V_{d o}-x_{q}^{\prime} I_{q o}
\end{aligned}
$$

Onde $V_{t o}, V_{q o}, V_{d o}, I_{t o}, I_{q o}, I_{d o}, \phi_{o}$ e $\delta_{o}$ são valores de regime permanente.

Para que a metodologia seja satisfatória a estimativa inicial para $E_{q o}^{\prime}$ e $E_{d o}^{\prime}$ deve ser próxima dos valores verdadeiros. Entretanto as estimativas para $E_{d o}^{\prime}$ e $E_{q o}$ obtidas através das equações 6.41 não são muito boas pois dependem dos parâmetros que são incertos $\left(x_{d}^{\prime}\right.$ e $\left.x_{q}^{\prime}\right)$ e das correntes e tensões calculadas em regime permanente. Utilizando os valores iniciais dos parâmetros distantes em $+30 \%$ dos valores reais e as estimativas iniciais de $E_{d o}^{\prime}$ e $E_{q o}^{\prime}$ em $+50 \%$ o procedimento não converge. 


\subsubsection{Teste $\mathrm{N}^{\circ}$ 3: Estimação de parâmetros da máquina síncrona considerando $E_{f d}$ como entrada e $\delta$ e $w$ como saída}

Neste teste escolheu-se a tensão do sistema de excitação como entrada do modelo matemático e o ângulo do rotor e a velocidade angular da máquina como saídas. As equações do modelo matemático e do modelo de sensibilidade são as mesmas àquelas utilizadas no teste $\mathrm{n}^{\circ} 2$, a única diferença está na matriz de sensibilidade que agora terá duas linha (a primeira linha está em relação ao ângulo e a segunda em relação à velocidade angular). Na figura 6.28 mostra-se o diagrama esquemático com as entradas e saídas do modelo matemático.

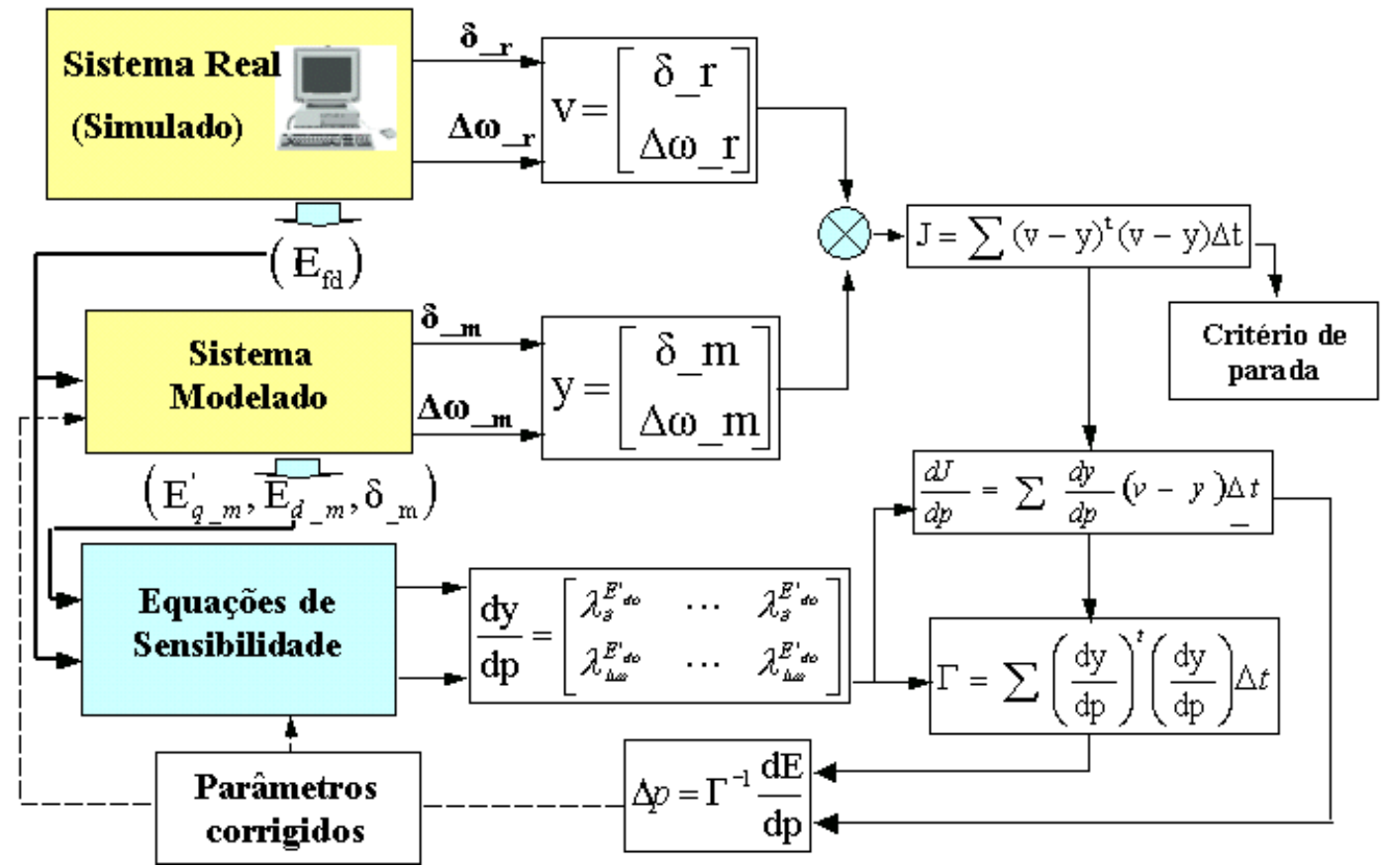

Figura 6.28: Diagrama esquemático considerando duas saídas

Na tabela 6.8 mostra-se os resultados obtidos após a aplicação da metodologia considerando que os valores iniciais dos parâmetros estão distantes em 15\% dos valores verdadeiros.

Estimativas iniciais dos parâmetros mais distantes de $15 \%$ dos valores verdadeiros levam a metodologia a divergência. No próximo teste incorpora-se a técnica de sincronização no processo de estimação de parâmetros. Com isto 
Tabela 6.8: Estimação dos parâmetros da máquina síncrona no teste $\mathrm{n}^{\circ} 3$.

\begin{tabular}{|c|c|c|c|c|}
\hline parâmetro & V. Inicial & V. final & V. Verdadeiro & Erro(\%) \\
\hline$x_{d}$ & 2.0815 & 1.8100 & 1.8100 & 0.0 \\
\hline$x_{d}^{\prime}$ & 0.3450 & 0.3000 & 0.300 & 0.0 \\
\hline$T_{d o}^{\prime}$ & 9.2000 & 7.9998 & 8.0000 & 0.0025 \\
\hline$x_{q}$ & 2.0240 & 1.7601 & 1.7600 & -0.073 \\
\hline$x_{q}^{\prime}$ & 0.7475 & 0.6500 & 0.6500 & 0.0 \\
\hline$T_{q o}^{\prime}$ & 1.1500 & 1.000 & 1.000 & 0.0 \\
\hline$E_{q o}^{\prime}$ & 1.3411 & 1.1662 & 1.1662 & 0.0 \\
\hline$E_{d o}^{\prime}$ & -0.3577 & -0.3110 & -0.3110 & 0.0 \\
\hline
\end{tabular}

os parâmetros são estimados com sucesso com maiores faixas de variação dos parâmetros.

\subsubsection{Teste $\mathrm{N}^{\circ}$ 4: Estimação considerando com o conceito de sincronização}

As condições para este teste são similares às condições do teste anterior. $E_{f d}$ é escolhida como entrada e como saídas $\delta$ e $w$. Além disso $\delta$ também é considerada como entrada de sincronização.Os sub-índices 'm' e 'r' são utilizados para diferençar as variáveis do 'modelo matemático' e do 'sistema real' respectivamente. Na figura 6.29 mostra-se o diagrama esquemático com as entradas e saídas para o 'modelo matemático'.

Dependendo da escolha das variáveis de entrada as equações diferenciais desaparecem da modelagem da máquina síncrona. Porém considerando variáveis como entradas de sincronização as equações diferenciais permanecem na modelagem. Portanto a metodologia tem um comportamento diferente. 


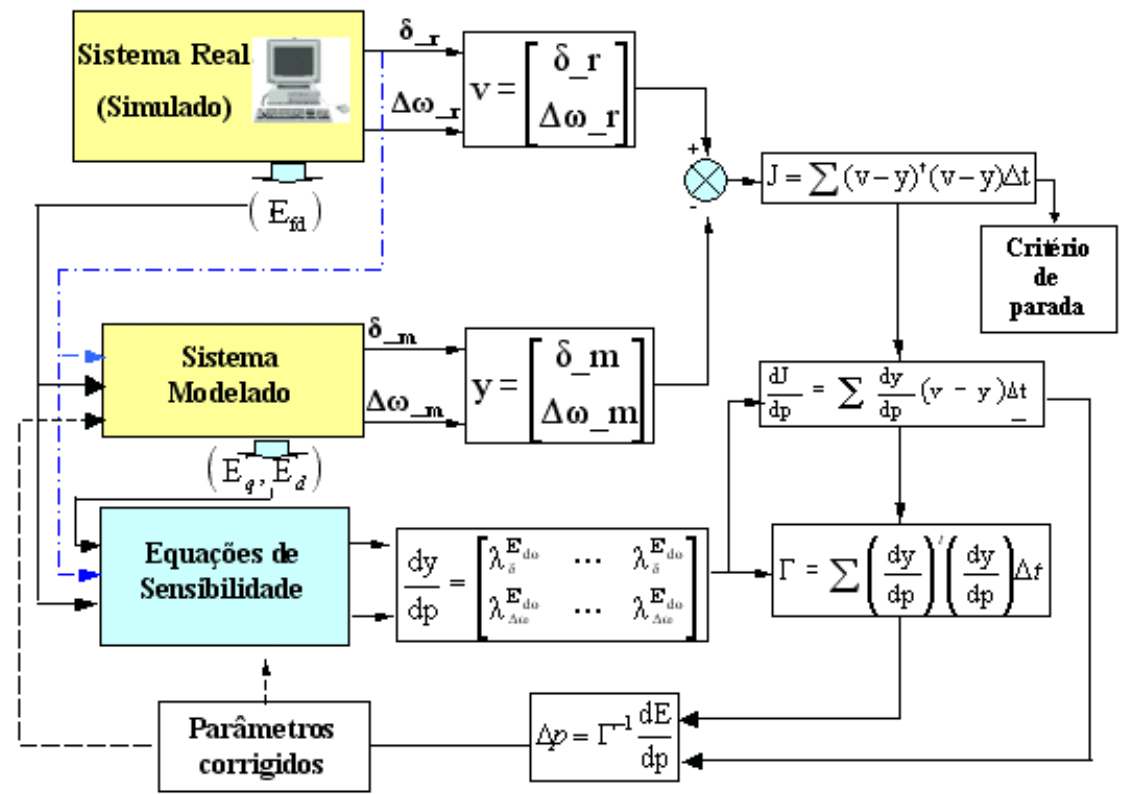

Figura 6.29: Diagrama esquemático considerando duas saídas

As equações do modelo matemático neste caso são as seguintes:

$$
\begin{aligned}
\dot{\delta_{m}} & =\omega_{m} \\
\dot{w_{m}} & =\frac{\omega_{o}}{2 H}\left[P_{m}-E_{q m}^{\prime} I_{q}-E_{d m}^{\prime} I_{d}-\left(x_{d}-x_{q}^{\prime}\right) I_{d} I_{q}-\frac{D}{\omega_{o}} \omega_{m}\right] \\
E_{q m}^{i} & =\frac{1}{T_{d o}^{\prime}}\left[E_{f d}-E_{q}^{\prime}+\left(x_{d}-x_{d}^{\prime}\right) I_{d}\right] \\
E_{d m}^{i} & =\frac{-1}{T_{q o}^{\prime}}\left[E_{d m}^{\prime}+\left(x_{q}-x_{q}^{\prime}\right) I_{q}\right]
\end{aligned}
$$

Note-se que como $\delta$ é entrada de sincronização a equação diferencial correspondente a esta variável permanece na modelagem. As correntes $I_{d}$ e $I_{q}$ são atualizadas em cada iteração através das equações do modelo matemático como:

$$
\begin{aligned}
I_{d} & =\frac{E_{b} \cos \left(\delta_{r}\right)-E_{q m}^{\prime}}{x_{d}^{\prime}+Z_{I}} \\
I_{d} & =\frac{E_{b} \operatorname{sen}\left(\delta_{r}\right)+E_{d m}^{\prime}}{x_{q}^{\prime}+Z_{I}}
\end{aligned}
$$

As correntes $I_{d}$ e $I_{q}$ no teste $\mathrm{n}^{\circ} 3$ estavam em função de $\delta_{m}$, porém, neste caso, estão em função de $\delta_{r}$.

O vetor de parâmetros é o seguinte:

$$
p=\left[\begin{array}{llllllll}
x_{d} & x_{d}^{\prime} & T_{d o}^{\prime} & x_{q} & x_{q}^{\prime} & T_{q o}^{\prime} & E_{q o}^{\prime} & E_{d o}^{\prime}
\end{array}\right]^{t} .
$$


As funções de sensibilidade são obtidas derivando-se as equações do modelo matemático em relação a cada um dos parâmetros e integrando-as no tempo. Para facilitar a análise, substitui-se $\frac{\partial f}{\partial p_{i}}$ por $\lambda_{f}^{p_{i}}$.

Derivando as equações do modelo matemático em relação a $x_{d}$ tem-se:

$$
\begin{aligned}
\dot{\lambda}_{\delta_{m}}^{x_{d}}= & \lambda_{w_{m}}^{x_{d}} \\
\dot{\lambda}_{w_{m}}^{x_{d}}= & \frac{w_{o}}{2 H}\left(-\lambda_{E_{q m}^{\prime}}^{x_{d}} I_{q}-E_{q m}^{\prime} \frac{\partial I_{q}}{\partial x_{d}}-\lambda_{E_{d m}^{\prime}}^{x_{d}} I_{d}-E_{d m}^{\prime} \frac{\partial I_{d}}{\partial x_{d}}-\left(x_{d}^{\prime}-x_{q}^{\prime}\right) \frac{\partial I_{d}}{\partial x_{d}} I_{q}\right. \\
& \left.-\left(x_{d}^{\prime}-x_{q}^{\prime}\right) \frac{\partial I_{q}}{\partial x_{d}} I_{d}-\frac{D}{w_{o}} \lambda_{w_{m}}^{x_{d}}\right) \\
\dot{\lambda}_{E_{q m}^{\prime}}^{x_{d}}= & \frac{1}{T_{d o}^{\prime}}\left(-\lambda_{E_{q m}^{\prime}}^{x_{d}}+I_{d}+\left(x_{d}-x_{d}^{\prime}\right) \frac{\partial I_{d}}{\partial x_{d}}\right) \\
\dot{\lambda}_{E_{d m}^{\prime}}^{x_{d}}= & -\frac{1}{T_{q o}^{\prime}}\left(\lambda_{E_{d m}^{\prime}}^{x_{d}}+\left(x_{q}-x_{q}^{\prime}\right) \frac{\partial I_{q}}{\partial x_{d}}\right)=0
\end{aligned}
$$

sendo:

$$
\begin{array}{ll}
\frac{\partial I_{q}}{\partial x_{d}}= & \frac{\lambda_{E_{d m}^{\prime}}^{x_{d}}}{x_{q}^{\prime}+Z_{I}}=0 \\
\frac{\partial I_{d}}{\partial x_{d}}= & -\frac{\lambda_{E_{q m}^{\prime}}^{x_{d}}}{x_{d}^{\prime}+Z_{I}}
\end{array}
$$

Derivando as equações do modelo matemático em relação a $x_{d}^{\prime}$

$$
\begin{aligned}
\dot{\lambda}_{\delta_{m}}^{x_{d}^{\prime}}= & \lambda_{w_{m}}^{x_{d}^{\prime}} \\
\dot{\lambda}_{w_{m}}^{x_{d}^{\prime}}= & \frac{w_{o}}{2 H}\left(-\lambda_{E_{q m}^{\prime}}^{x_{d}^{\prime}} I_{q}-E_{q m}^{\prime} \frac{\partial I_{q}}{\partial x_{d}^{\prime}}-\lambda_{E_{d m}^{\prime}}^{x_{d}^{\prime}} I_{d}-E_{d m}^{\prime} \frac{\partial I_{d}}{\partial x_{d}^{\prime}}-I_{d} I_{q}+\right. \\
& \left.-\left(x_{d}^{\prime}-x_{q}^{\prime}\right) \frac{\partial I_{q}}{\partial x_{d}^{\prime}} I_{d}-\left(x_{d}^{\prime}-x_{q}^{\prime}\right) \frac{\partial I_{d}}{\partial x_{d}^{\prime}} I_{q}-\frac{D}{w_{o}} \lambda_{w_{m}}^{x_{d}^{\prime}}\right) \\
\dot{\lambda}_{E_{q m}^{\prime}}^{x_{d}^{\prime}}= & \frac{1}{T_{d o}^{\prime}}\left(-\lambda_{E_{q m}^{\prime}}^{x_{d}}+I_{d}+\left(x_{d}-x_{d}^{\prime}\right) \frac{\partial I_{d}}{\partial x_{d}^{\prime}}\right) \\
\dot{\lambda}_{E_{d m}^{\prime}}^{x_{d}^{\prime}}= & -\frac{1}{T_{q o}^{\prime}}\left(\lambda_{E_{d m}^{\prime}}^{x_{d}^{\prime}}+\left(x_{q}-x_{q}^{\prime}\right) \frac{\partial I_{q}}{\partial x_{d}^{\prime}}\right)=0
\end{aligned}
$$

sendo:

$$
\begin{array}{ll}
\frac{\partial I_{q}}{\partial x_{d}^{\prime}}= & \frac{\lambda_{E_{d}^{\prime}}^{x^{\prime}}}{x_{q}^{\prime}+Z_{I}}=0 \\
\frac{\partial I_{d}}{\partial x_{d}^{\prime}}= & -\frac{\lambda_{E_{q}^{\prime}}^{x^{\prime}}}{x_{d}^{\prime}+Z_{I}}-\frac{E_{b} \cos \left(\delta_{r}\right)-E_{q}^{\prime}}{\left(x_{d}^{\prime}+Z_{I}\right)^{2}}
\end{array}
$$


Derivando as equações do modelo matemático em relação a $T_{d o}^{\prime}$

$$
\begin{aligned}
\dot{\lambda}_{\delta_{m}}^{T_{d o}^{\prime}=} & \lambda_{w_{m}}^{T_{d o}^{\prime}} \\
\dot{\lambda}_{w_{m}}^{T_{d o}^{\prime}}= & \frac{w_{o}}{2 H}\left(-\lambda_{E_{q m}^{\prime}}^{x_{d}^{\prime}} I_{q}-E_{q m}^{\prime} \frac{\partial I_{q}}{\partial T_{d o}^{\prime}}-\lambda_{E_{d m}^{\prime}}^{T_{d o}^{\prime}} I_{d}-E_{d m}^{\prime} \frac{\partial I_{d}}{\partial T_{d o}^{\prime}}-\left(x_{d}^{\prime}-x_{q}^{\prime}\right) \frac{\partial I_{q}}{\partial T_{d o}^{\prime}} I_{d}+\right. \\
& \left.-\left(x_{d}^{\prime}-x_{q}^{\prime}\right) \frac{\partial I_{d}}{\partial T_{d o}^{\prime}} I_{q}-\frac{D}{w_{o}} \lambda_{w_{m}}^{T_{d o}^{\prime}}\right) \\
\dot{\lambda}_{E_{q m}^{\prime}}^{T_{d o}^{\prime}}= & -\frac{1}{T_{d o}^{\prime 2}}\left(E_{f d}-E_{q}^{\prime}+\left(x_{d}-x_{q}^{\prime}\right) I_{d}\right)+\frac{1}{T_{d o}}\left(-\lambda_{E_{q m}^{\prime}}^{T_{d o}^{\prime}}+\left(x_{d}-x_{d}^{\prime}\right) \frac{\partial I_{d}}{\partial T_{d o}^{\prime}}\right) \\
\dot{\lambda}_{E_{d m}^{\prime}}^{T_{d o}^{\prime}}= & -\frac{1}{T_{q o}^{\prime}}\left(\lambda_{E_{d m}^{\prime}}^{T_{d o}^{\prime}}+\left(x_{q}-x_{q}^{\prime}\right) \frac{\partial I_{q}}{\partial T_{d o}^{\prime}}\right)=0
\end{aligned}
$$

sendo:

$$
\begin{array}{ll}
\frac{\partial I_{q}}{\partial T_{d o}^{\prime}}= & \frac{\lambda_{E_{d}^{\prime}}^{T_{d o}^{\prime}}}{x_{q}^{\prime}+Z_{I}}=0 \\
\frac{\partial I_{d}}{\partial T_{d o}^{\prime}}= & -\frac{\lambda_{E_{q}^{\prime}}^{T_{d o}^{\prime}}}{x_{d}^{\prime}+Z_{I}}
\end{array}
$$

Derivando as equações do modelo matemático em relação a $x_{q}$

$$
\begin{aligned}
\dot{\lambda}_{\delta_{m}}^{x_{q}}= & \lambda_{w_{m}}^{x_{q}} \\
\dot{\lambda}_{w_{m}}^{x_{q}}= & \frac{w_{o}}{2 H}\left(-\lambda_{E_{q m}^{\prime}}^{x_{q}} I_{q}-E_{q m}^{\prime} \frac{\partial I_{q}}{\partial x_{q}}-\lambda_{E_{d m}^{\prime}}^{x_{q}} I_{d}-E_{d m}^{\prime} \frac{\partial I_{d}}{\partial x_{q}}-\left(x_{d}^{\prime}-x_{q}^{\prime}\right) \frac{\partial I_{d}}{\partial x_{q}} I_{q}+\right. \\
& \left.-\left(x_{d}^{\prime}-x_{q}^{\prime}\right) \frac{\partial I_{q}}{\partial x_{q}} I_{d}-\frac{D}{w_{o}} \lambda_{w_{m}}^{x_{q}}\right) \\
\dot{\lambda}_{E_{q m}^{\prime}}^{x_{q}}= & \frac{1}{T_{d o}^{\prime}}\left(-\lambda_{E_{q m}^{\prime}}^{x_{q}}+\left(x_{d}-x_{d}^{\prime}\right) \frac{\partial I_{d}}{\partial x_{q}}\right)=0 \\
\dot{\lambda}_{E_{d m}^{\prime}}^{x_{q}}= & -\frac{1}{T_{q o}^{\prime}}\left(\lambda_{E_{d m}^{\prime}}^{x_{q}}+I_{q}+\left(x_{q}-x_{q}^{\prime}\right) \frac{\partial I_{q}}{\partial x_{q}}\right)
\end{aligned}
$$

sendo:

$$
\begin{array}{ll}
\frac{\partial I_{q}}{\partial x_{q}}= & \frac{\lambda_{E_{d m}^{\prime}}^{x_{q}}}{x_{q}^{\prime}+Z_{I}} \\
\frac{\partial I_{d}}{\partial x_{q}}= & -\frac{\lambda_{E_{q m}^{\prime}}^{x_{q}}}{x_{d}^{\prime}+Z_{I}}=0
\end{array}
$$


Derivando as equações do modelo matemático em relação a $x_{q}^{\prime}$

$$
\begin{aligned}
\dot{\lambda}_{\delta_{m}}^{x_{q}^{\prime}}= & \lambda_{w_{m}}^{x_{q}^{\prime}} \\
\dot{\lambda}_{w_{m}}^{x_{q}^{\prime}}= & \frac{w_{o}}{2 H}\left(-\lambda_{E_{q m}^{\prime}}^{x_{q}^{\prime}} I_{q}-E_{q m}^{\prime} \frac{\partial I_{q}}{\partial x_{q}^{\prime}}-\lambda_{E_{d m}^{\prime}}^{x_{q}^{\prime}} I_{d}-E_{d m}^{\prime} \frac{\partial I_{d}}{\partial x_{q}^{\prime}}+I_{q} I_{d}+\right. \\
& \left.-\left(x_{d}^{\prime}-x_{q}^{\prime}\right) \frac{\partial I_{d}}{\partial x_{q}^{\prime}} I_{q}-\left(x_{d}^{\prime}-x_{q}^{\prime}\right) \frac{\partial I_{q}}{\partial x_{q}^{\prime}} I_{d}-\frac{D}{w_{o}} \lambda_{w_{m}}^{x_{q}^{\prime}}\right) \\
\dot{\lambda}_{E_{q m}^{\prime}}^{x^{\prime}}= & \frac{1}{T_{d o}^{\prime}}\left(-\lambda_{E_{q m}^{\prime}}^{x^{\prime}}+\left(x_{d}-x_{d}^{\prime}\right) \frac{\partial I_{d}}{\partial x_{q}^{\prime}}\right)=0 \\
\dot{\lambda}_{E_{d m}^{\prime}}^{x^{\prime}}= & -\frac{1}{T_{q o}^{\prime}}\left(\lambda_{E_{d m}^{\prime}}^{x^{\prime}}-I_{q}+\left(x_{q}-x_{q}^{\prime}\right) \frac{\partial I_{q}}{\partial x_{q}^{\prime}}\right)=0
\end{aligned}
$$

sendo:

$$
\begin{aligned}
\frac{\partial I_{q}}{\partial x_{q}} & =\quad \frac{\lambda_{E_{d m}^{\prime}}^{x_{q}}}{x_{q}^{\prime}+Z_{I}}-\frac{E_{b} \operatorname{sen}\left(\delta_{r}\right)+E_{d}^{\prime}}{\left(x_{q}^{\prime}+Z_{I}\right)^{2}} \\
\frac{\partial I_{d}}{\partial x_{q}^{\prime}} & =-\frac{\lambda_{E_{q m}^{\prime}}^{x^{\prime}}}{x_{d}^{\prime}+Z_{I}}=0
\end{aligned}
$$

Derivando as equações do modelo matemático em relação a $T_{q o}^{\prime}$

$$
\begin{aligned}
\dot{\lambda}_{\delta_{m}}^{T_{q o}^{\prime}=} & \lambda_{w_{m}}^{T_{q o}^{\prime}} \\
\dot{\lambda}_{w_{m}}^{T_{q o}^{\prime}=} & \frac{w_{o}}{2 H}\left(-\lambda_{E_{q m}^{\prime}}^{T_{q o}^{\prime}} I_{q}-E_{q m}^{\prime} \frac{\partial I_{q}}{\partial T_{q o}^{\prime}}-\lambda_{E_{d m}^{\prime}}^{T_{q o}^{\prime}} I_{d}-E_{d m}^{\prime} \frac{\partial I_{d}}{\partial T_{q o}^{\prime}}+\right. \\
& \left.-\left(x_{d}^{\prime}-x_{q}^{\prime}\right) \frac{\partial I_{d}}{\partial T_{q o}^{\prime}} I_{q}-\left(x_{d}^{\prime}-x_{q}^{\prime}\right) \frac{\partial I_{q}}{\partial x_{q}^{\prime}} I_{d}-\frac{D}{w_{o}} \lambda_{w_{m}}^{T_{q o}^{\prime}}\right) \\
\dot{\lambda}_{E_{q m}^{\prime}}^{T_{q o}^{\prime}}= & \frac{1}{T_{d o}^{\prime}}\left(-\lambda_{E_{q m}^{\prime}}^{T_{q o}^{\prime}}+\left(x_{d}-x_{d}^{\prime}\right) \frac{\partial I_{d}}{\partial T_{q o}^{\prime}}\right)=0 \\
\dot{\lambda}_{E_{d m}^{\prime}}^{T_{q o}^{\prime}}= & \frac{1}{T_{q o}^{\prime 2}}\left(E_{d m}^{\prime 2}+\left(x_{q}-x_{q}^{\prime}\right) I_{q}\right)-\frac{1}{\partial T_{q o}^{\prime}}\left(\lambda_{E_{d}^{\prime}}^{T_{q}^{\prime}}+\left(x_{q}-x_{q}^{\prime}\right) \frac{\partial I_{q}}{\partial T_{q o}^{\prime}}\right)
\end{aligned}
$$

sendo:

$$
\begin{aligned}
\frac{\partial I_{q}}{\partial T_{q o}} & =\frac{\lambda_{E_{d m}^{\prime}}^{T_{q o}}}{x_{q}^{\prime}+Z_{I}} \\
\frac{\partial I_{d}}{\partial T_{q o}^{\prime}} & =-\frac{\lambda_{E_{q m}^{\prime}}^{T^{\prime}}}{x_{d}^{\prime}+Z_{I}}=0
\end{aligned}
$$

As condições iniciais em $\mathrm{t}=t_{o}$, para todas as equações diferenciais mostradas anteriormente são iguais a zero, assim as soluções de algumas equações diferenciais são zero (isto é mostrado explicitamente). As derivadas das equações do modelo 
matemático em relação ao parâmetro $E_{q o}^{\prime}$ são:

$$
\begin{aligned}
\dot{\lambda}_{\delta_{m}}^{E_{q o}^{\prime}}= & \lambda_{w_{m}}^{E_{q o}^{\prime}} \\
\dot{\lambda}_{w_{m}}^{E_{q o}^{\prime}}= & \frac{w_{o}}{2 H}\left(-\lambda_{E_{q m}^{\prime}}^{E_{q o}^{\prime}} I_{q}-E_{q m}^{\prime} \frac{\partial I_{q}}{\partial E_{q o}^{\prime}}-\lambda_{E_{d m}^{\prime}}^{E_{q o}^{\prime}} I_{d}-E_{d m}^{\prime} \frac{\partial I_{d}}{\partial E_{q o}^{\prime}}+\right. \\
& \left.-\left(x_{d}^{\prime}-x_{q}^{\prime}\right) \frac{\partial I_{d}}{\partial E_{q o}^{\prime}} I_{d}-\left(x_{d}^{\prime}-x_{q}^{\prime}\right) \frac{\partial I_{d}}{\partial E_{q o}^{\prime}} I_{q}-\frac{D}{w_{o}} \lambda_{w_{m}}^{E_{q o}^{\prime}}\right) \\
\dot{\lambda}_{E_{q m}^{\prime}}^{E_{q o}^{\prime}}= & \frac{1}{T_{d o}^{\prime}}\left(-\lambda_{E_{q m}^{\prime}}^{E_{q o}^{\prime}}+\left(x_{d}-x_{d}^{\prime}\right) \frac{\partial I_{d}}{\partial E_{q o}^{\prime}}\right)=0 \\
\dot{\lambda}_{E_{d m}^{\prime}}^{E_{q o}^{\prime}}= & -\frac{1}{T_{q o}^{\prime}}\left(\lambda_{E_{d m}^{\prime}}^{E_{q o}^{\prime}}\left(x_{q}-x_{q}^{\prime}\right) \frac{\partial I_{q}}{\partial E_{q o}^{\prime}}\right)
\end{aligned}
$$

sendo:

$$
\begin{aligned}
\frac{\partial I_{q}}{\partial E_{q o}} & =\frac{\lambda_{E_{d m}^{\prime}}^{E_{q o}}}{x_{q}^{\prime}+Z_{I}}=0 \\
\frac{\partial I_{d}}{\partial E_{q o}^{\prime}} & =-\frac{\lambda_{E_{q o}^{\prime}}^{E^{\prime}}}{x_{d}^{\prime}+Z_{I}}
\end{aligned}
$$

com condições iniciais:

$$
\lambda_{\delta_{m}}^{E_{q o}^{\prime}}=0 \quad \lambda_{w_{m}}^{E_{q o}^{\prime}}=0 \quad \lambda_{E_{q m}^{\prime}}^{E_{q o}^{\prime}}=1 \quad \lambda_{E_{d m}^{\prime}}^{E_{q o}^{\prime}}=0
$$

Em relação a $E_{d o}^{\prime}$ temos:

$$
\begin{aligned}
\dot{\lambda}_{\delta_{m}}^{E_{d o}^{\prime}}= & \lambda_{w_{m}}^{E_{d o}^{\prime}} \\
\dot{\lambda}_{w_{m}}^{E_{d o}^{\prime}}= & \frac{w_{o}}{2 H}\left(-\lambda_{E_{q m}^{\prime}}^{E_{d o}^{\prime}} I_{q}-E_{q m}^{\prime} \frac{\partial I_{q}}{\partial E_{d o}^{\prime}}-\lambda_{E_{d m}^{\prime}}^{E_{d o}^{\prime}} I_{d}-E_{d m}^{\prime} \frac{\partial I_{d}}{\partial E_{d o}^{\prime}}+\right. \\
& \left.-\left(x_{d}^{\prime}-x_{q}^{\prime}\right) \frac{\partial I_{d}}{\partial E_{d o}^{\prime}} I_{d}-\left(x_{d}^{\prime}-x_{q}^{\prime}\right) \frac{\partial I_{d}}{\partial E_{q o}^{\prime}} I_{q}-\frac{D}{w_{o}} \lambda_{w_{m}}^{E_{d o}^{\prime}}\right) \\
\dot{\lambda}_{E_{q m}^{\prime}}^{E_{d o}^{\prime}}= & \frac{1}{T_{d o}^{\prime}}\left(-\lambda_{E_{q m}^{\prime}}^{E_{d o}^{\prime}}+\left(x_{d}-x_{d}^{\prime}\right) \frac{\partial I_{d}}{\partial E_{d o}^{\prime}}\right)=0 \\
\dot{\lambda}_{E_{d m}^{\prime}}^{E_{d o}^{\prime}}= & -\frac{1}{T_{q o}^{\prime}}\left(\lambda_{E_{d m}^{\prime}}^{E_{d o}^{\prime}}\left(x_{q}-x_{q}^{\prime}\right) \frac{\partial I_{q}}{\partial E_{d o}^{\prime}}\right)
\end{aligned}
$$

sendo:

$$
\begin{aligned}
\frac{\partial I_{q}}{\partial E_{d o}} & =\frac{\lambda_{E_{d m}^{\prime}}^{E_{d o}}}{x_{q}^{\prime}+Z_{I}}=0 \\
\frac{\partial I_{d}}{\partial E_{d o}^{\prime}} & =-\frac{\lambda_{E_{q m}^{\prime}}^{E^{\prime}}}{x_{d}^{\prime}+Z_{I}}
\end{aligned}
$$

com condições iniciais:

$$
\lambda_{\delta_{m}}^{E_{d o}^{\prime}}=0 \quad \lambda_{w_{m}}^{E_{d o}^{\prime}}=0 \quad \lambda_{E_{q m}^{\prime}}^{E_{d o}^{\prime}}=1 \quad \lambda_{E_{d m}^{\prime}}^{E_{d o}^{\prime}}=0
$$


Os parâmetros verdadeiros foram alterados em $+20 \%$ e utilizados no modelo matemático como valores iniciais. As estimativas inicias para $E_{q o}^{\prime}$ e $E_{d o}^{\prime}$ foram obtidas das condições em regime permanente segundo o conjunto de equações 6.41. A estimativa obtida para $E_{q o}^{\prime}$ é aproximadamente a $+70 \%$ do valor verdadeiro e a estimativa para $E_{d o}^{\prime}$ corresponde a $+3 \%$ do valor verdadeiro (valores obtidos em pós-falta). Mesmo que a estimativa inicial para $E_{q o}^{\prime}$ esteja distante do valor verdadeiro a metodologia conseguiu estimar todos os parâmetros, porém é necessário encontrar alguma outra maneira de obter as estimativas inicias $E_{d o}^{\prime}$ e $E_{q o}$ pois o sucesso do processo de identificação depende de que estes parâmetros não fiquem muito distantes dos valores verdadeiros.

Na figura 6.30 compara-se o ângulo interno da máquina do sistema real e do modelo matemático antes do processo de estimação. Da mesma maneira, na figura 6.31, compara-se a velocidade angular do 'sistema real' e do 'modelo matemático'.
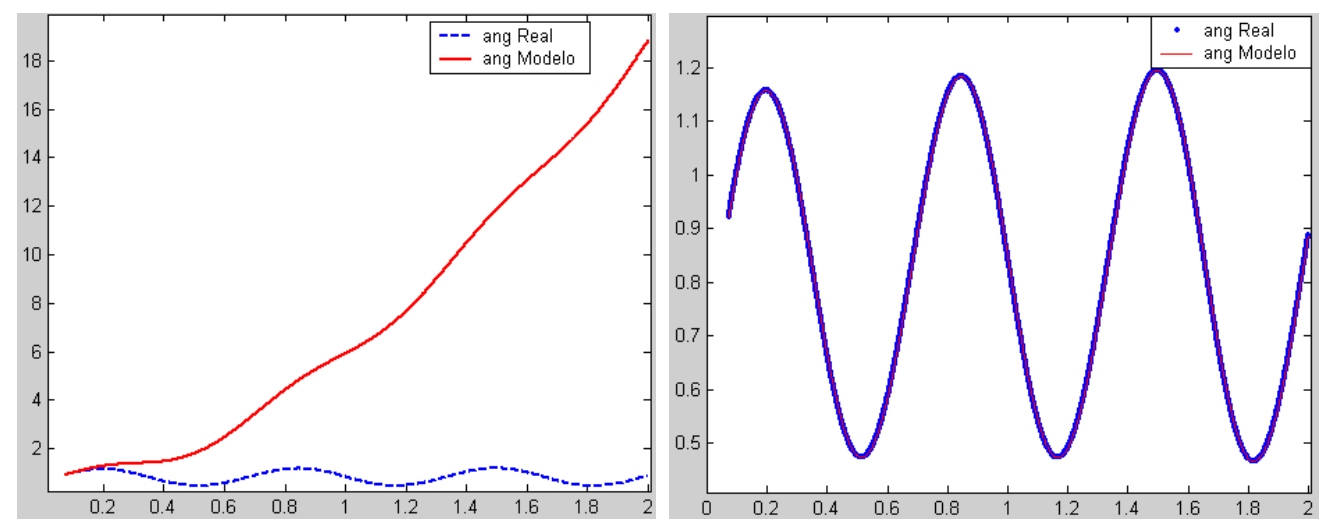

Figura 6.30: Ângulo do rotor antes e depois do processo de estimação dos parâmetros no teste $n^{\circ} 4$

Na figura 6.31, compara-se a velocidade angular do sistema real e do modelo matemático antes e depois da estimação dos parâmetros.

Na tabela 6.9 mostra-se o resumo dos resultados após a convergência dos parâmetros. Os parâmetros convergiram em 8 iterações. 

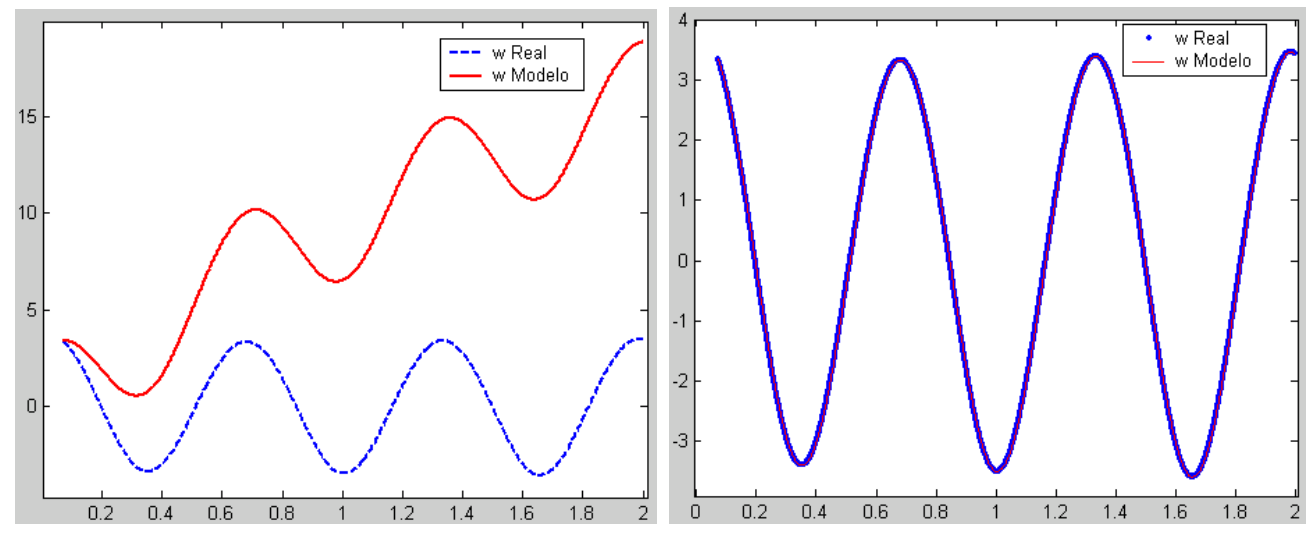

Figura 6.31: Velocidade do rotor antes e depois a estimação dos parâmetros no teste $n^{\circ} 4$

Tabela 6.9: Estimação de parâmetros da máquina síncrona no teste $\mathrm{n}^{\circ} 4$.

\begin{tabular}{|c|c|c|c|c|}
\hline parâmetro & V. Inicial & V. final & V. Real & Erro(\%) \\
\hline$x_{d}$ & 2.1720 & 1.8100 & 1.8100 & 0.0 \\
\hline$x_{d}^{\prime}$ & 0.3600 & 0.3000 & 0.300 & 0.0 \\
\hline$T_{d o}^{\prime}$ & 9.600 & 7.9998 & 8.0000 & 0.0025 \\
\hline$x_{q}$ & 2.1120 & 1.7587 & 1.7600 & -0.073 \\
\hline$x_{q}^{\prime}$ & 0.7800 & 0.6500 & 0.6500 & $0.0(\%)$ \\
\hline$T_{q o}^{\prime}$ & 1.200 & 1.000 & 1.000 & $0.0(\%)$ \\
\hline$E_{q o}^{\prime}$ & 1.1258 & 1.1662 & 1.1662 & $0.0(\%)$ \\
\hline$E_{d o}^{\prime}$ & -0.5302 & -0.3110 & -0.3110 & $0.0(\%)$ \\
\hline
\end{tabular}




\subsubsection{Estimação dos parâmetros mecânicos H e D}

A metodologia é aplicada para a estimação dos parâmetros da máquina síncrona 'H' e 'D'. Analisando as equações disponíveis viu-se que utilizando apenas a segunda equação da máquina síncrona (equação de velocidade angular) é possível identificar os dois parâmetros desejados pois esta equação depende de 'H' e 'D' que são os parâmetros a identificar. A variável de entrada escolhida é a potência elétrica e a variável de saída é a velocidade angular.

Para representar a variável de velocidade do modelo matemático é utilizado a variável ' $v$ ' para não gerar confusão com as variáveis do sistema real.

Para o 'sistema real' tem-se

$$
\dot{w}=\frac{w_{o}}{2 H}\left(P_{m}-P_{e}-\frac{D}{w_{o}} w\right)
$$

e para o modelo matemático

$$
\dot{v}=\frac{w_{o}}{2 H}\left(P_{m}-P_{e}-\frac{D}{w_{o}} v\right)
$$

Onde $w_{o}=2 \pi f$

As funções de sensibilidade são determinadas derivando a equação da saída do modelo matemático em relação aos parâmetros 'H' e 'D' e integrando-as no tempo.

Derivando a equação 6.43 e fazendo as seguintes substituições $\lambda_{v}^{H}=\frac{\partial \omega}{\partial H} \mathrm{e}$ $\lambda_{v}^{D}=\frac{\partial \omega}{\partial D}$ obtém-se:

$$
\left(\begin{array}{l}
\dot{\lambda}_{v}^{H} \\
\dot{\lambda}_{v}^{D}
\end{array}\right)=\left(\begin{array}{c}
-\frac{\omega_{o}}{H^{2}}\left(P_{m}-P_{e r}-\frac{D}{\omega_{o}} v\right)+\frac{\omega_{o}}{H}\left(-\frac{D}{\omega} \lambda_{v}^{H}\right) \\
\frac{\omega_{o}}{H}\left(-\frac{v}{\omega_{o}}\right)+\frac{\omega_{o}}{H}\left(-\frac{D}{\omega_{o}} \lambda_{v}^{D}\right)
\end{array}\right)
$$

O diagrama esquemático para a aplicação do processo de estimação é mostrado na figura 6.32 . Os parâmetros verdadeiros foram alterados em $80 \%$ e utilizados como estimativas inicias no 'modelo matemático'. A metodologia conseguiu estimar com sucesso os dois parâmetros mecânicos da máquina síncrona. Na tabela 6.10 mostram-se os valores ' $H$ ' e 'D' em cada iteração até a convergência dos parâmetros, e na tabela 6.11 apresentam-se o resumo dos resultados. 


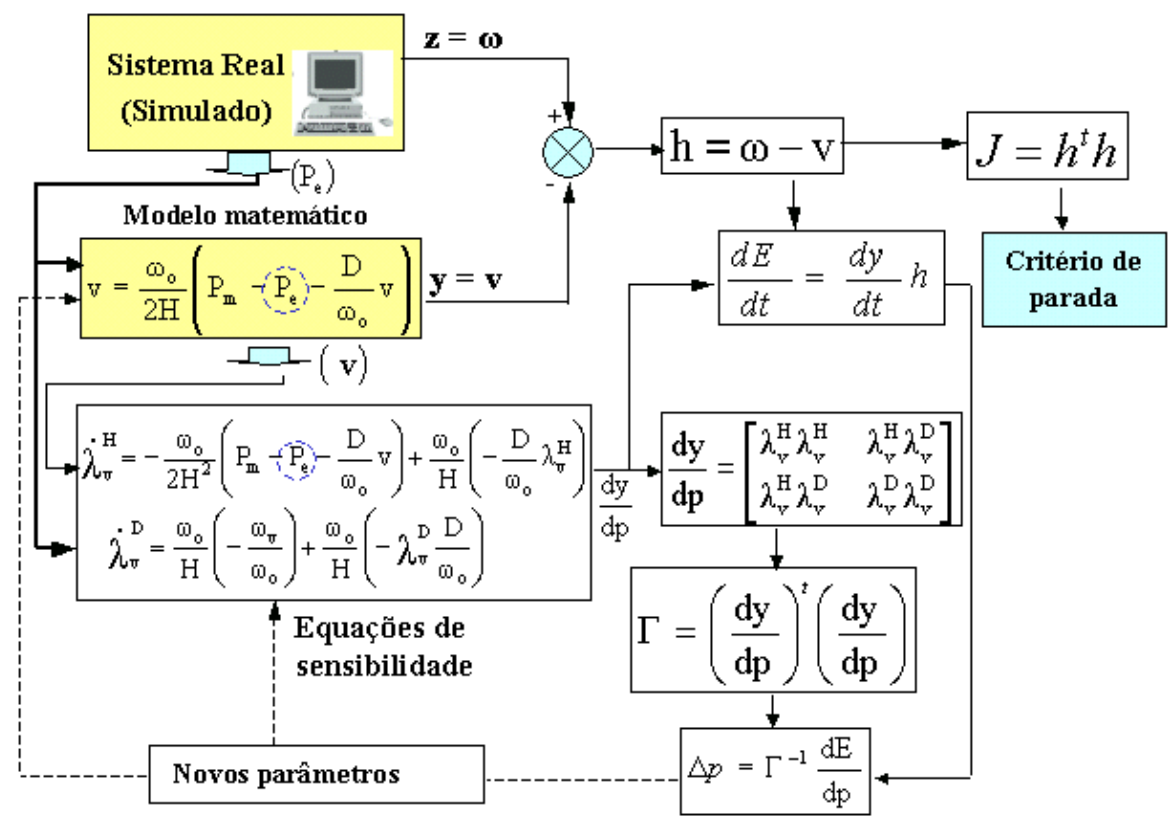

Figura 6.32: Diagrama esquemático para a estimação de H e D

Tabela 6.10: Estimação dos parâmetros mecânicos da máquina síncrona em cada iteração

\begin{tabular}{|c|c|c|}
\hline Iteração & $\mathrm{H}$ & $\mathrm{D}$ \\
\hline 0(V.Inicial) & 0.7 & 0.18 \\
\hline 1 & 1.2599 & 0.3241 \\
\hline 2 & 2.0659 & 0.5321 \\
\hline 3 & 2.9115 & 0.7521 \\
\hline 4 & 3.3995 & 0.8841 \\
\hline 5 & 3.4954 & 0.9077 \\
\hline
\end{tabular}

Tabela 6.11: Resumo dos resultados da estimação dos parâmetros da máquina síncrona

\begin{tabular}{|c|c|c|c|c|}
\hline parâmetro & V. Inicial & V. final & V. Real & Erro(\%) \\
\hline H. & 0.18 & 0.9077 & 0.9 & $0.49(\%)$ \\
\hline D. & 0.7 & 3.4954 & 3.5 & $-0.13(\%)$ \\
\hline
\end{tabular}




\subsection{Parâmetros do Sistema de Excitação}

\subsubsection{Estimação do sistema de excitação ST1A}

A metodologia de sensibilidade da trajetória é aplicada ao sistema de excitação ST1A apresentado no capítulo 4. A equação diferencial para este modelo foi apresentada na seção 5.2 e é mostrada novamente aqui para facilitar a referência

$$
\frac{d E_{f d m}}{d t}=\frac{1}{T_{R}}\left(K_{A}\left(V_{\text {ref }}-V_{t}\right)-E_{f d m}\right)
$$

A ação dos limites do sistema de excitação é considerada através das seguintes restrições:

- Se $E_{f d m}>E_{f d m a x}: E_{f d m}=E_{f d \max }$

- Se $E_{f d m}<E_{f d m i n}: E_{f d m}=E_{f d m i n}$

O sub-índice 'm' denota variável do modelo matemático. Os valores verdadeiros dos parâmetros são: $T_{R}=0.02$ e $K_{A}=200$. Estes valores são utilizados para simular computacionalmente o sistema real. Estes valores foram alterados em -40\% e utilizados para simular o modelo matemático. As equações de sensibilidade são obtidas derivando-se a equação 6.44 em relação aos parâmetros $T_{R}$ e $K_{A}$ :

$$
\begin{aligned}
& \dot{\lambda}_{E_{f d}}^{T_{R}}=-\frac{1}{T_{R}^{2}}\left(K_{A}\left(V_{\text {ref }}-V_{t}\right)-E_{f d}\right)+\frac{1}{T_{R}}\left(-\lambda_{E_{f d}}^{T_{R}}\right) \\
& \dot{\lambda}_{E_{f d}}^{K_{A}}=\frac{1}{T_{R}}\left[\left(V_{\text {ref }}-V_{t}\right)-\lambda_{E_{f d}}^{K_{A}}\right]
\end{aligned}
$$

A variável de entrada escolhida é a tensão terminal da máquina e a variável de saída é a tensão de campo. Isto é mostrado na figura (6.33). O teste é similar aos casos anteriores. Simula-se uma falta trifásica na linha 2 (L-2) perto da barra 1, a falta é eliminada pela atuação dos dispositivos de proteção isolando a linha em curto no tempo de $\mathrm{t}=0.07 \mathrm{~s}$.

A tensão do sistema de excitação antes e depois do processo de estimação dos parâmetros são mostradas na figura 6.34). O período de amostragem é $T=\left[\begin{array}{ll}0 & 2.5\end{array}\right]$ s, e o passo de integração utilizado foi de $2.5 \mathrm{~ms}$. Os resultados da 


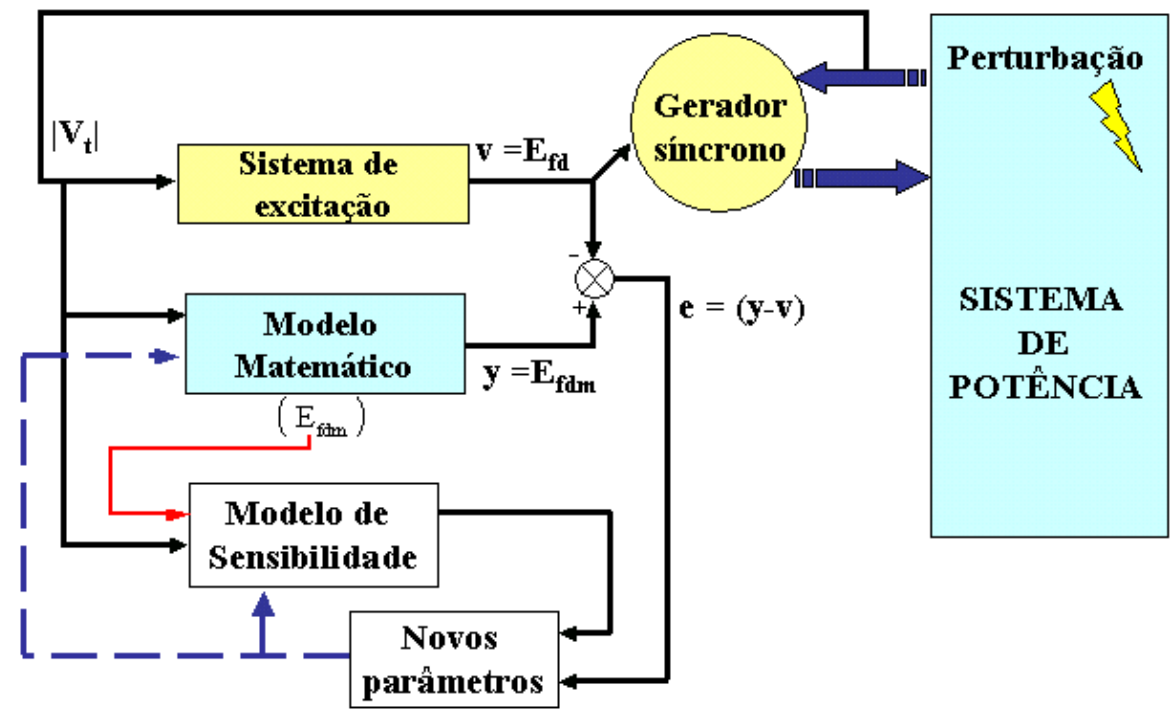

Figura 6.33: Diagrama esquemático para estimação dos parâmetros do sistema excitação ST1A.
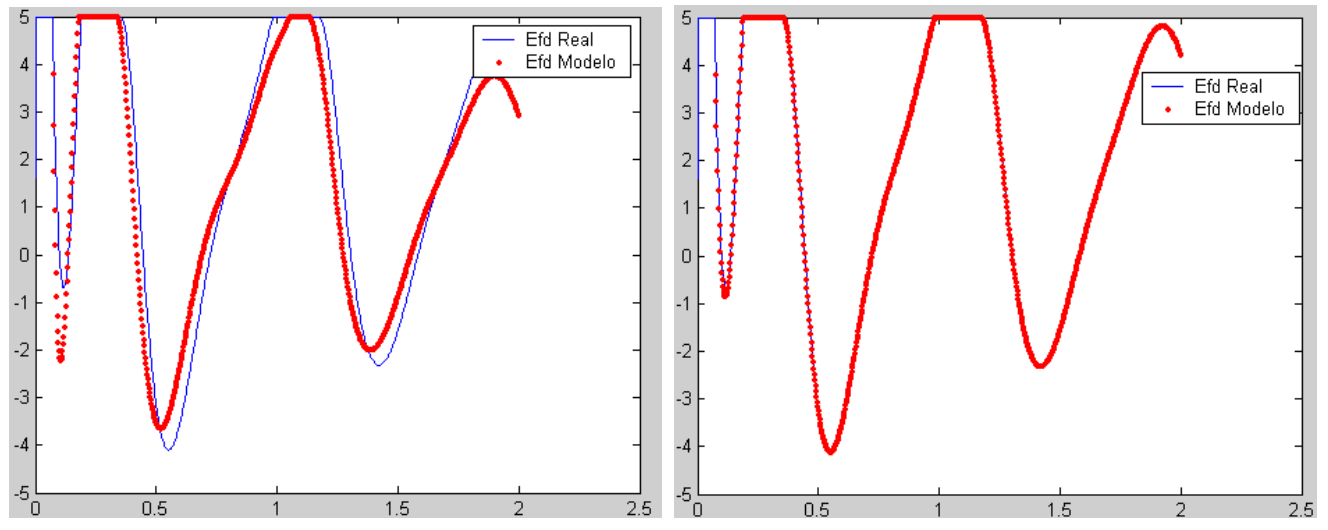

Figura 6.34: $E_{f d}$ antes e depois a estimação dos parâmetros

estimação dos parâmetros são apresentados na tabela 6.12. Os parâmetros foram estimados com um erro inferior a $3 \%$.

Tabela 6.12: Estimação dos parâmetros do sistema de excitação ST1A.

\begin{tabular}{|c|c|c|c|c|}
\hline parâmetro & V. Inicial & V. Final & V. Verdadeiro & Erro(\%) \\
\hline$K_{A}$ & 150 & 199.32 & 200 & -0.34 \\
\hline$T_{R}$ & 0.02 & 0.0487 & 0.05 & -2.6 \\
\hline
\end{tabular}




\subsubsection{Estimação dos parâmetros do sistema de excitação DC1}

A metodologia de sensibilidade da trajetória é aplicada ao sistema de excitação tipo DC1 apresentado no capítulo 4 (vide figura 5.11). As equações diferenciais para o modelo matemático são as seguintes:

$$
\begin{aligned}
\frac{d E_{v}}{d t} & =\frac{1}{T_{R}}\left(V_{t}-E_{v}\right) \\
\frac{d E_{i i i}}{d t} & =\frac{1}{T_{A}}\left(K_{A}\left(V_{r e f}-E_{i v}-E_{v}\right)-E_{i i i}\right) \\
\frac{d E_{f d m}}{d t} & =\frac{1}{T_{E}}\left(E_{i i i}-\left(A e^{B E_{f d m}}+K_{E}\right) E_{f d m}\right) \\
\frac{d E_{i v}}{d t} & =\frac{1}{T_{F}}\left(K_{F} \frac{d E_{f d}}{d t}-E_{i v}\right)
\end{aligned}
$$

O sub-índice 'm' utilizado na tensão de campo denota que é uma variável do modelo matemático. O vetor dos parâmetros a serem estimados é dado por:

$$
p=\left[\begin{array}{lllllllll}
T_{R} & T_{A} & K_{A} & T_{E} & K_{E} & A & B & T_{F} & K_{F}
\end{array}\right]^{t}
$$

O vetor de parâmetros com os valores verdadeiros é dado por:

$$
p_{r}=\left[\begin{array}{lllllllll}
0.015 & 0.06 & 50 & 0.052 & -0.0385 & 0.0012 & 1.264 & 1 & 0.0832
\end{array}\right]^{t}
$$

Derivando as equações diferenciais do modelo matemático em relação aos parâmetros e fazendo $\lambda_{f_{i}}^{p_{i}}=\frac{\partial f_{i}}{p_{i}}$ tem-se:

Derivando o conjunto de equações 6.45 em relação a $T_{R}$

$$
\begin{aligned}
\dot{\lambda}_{E_{v}}^{T_{R}} & =-\frac{1}{T_{R}^{2}}\left(V_{t}-E_{v}\right)+\frac{1}{T_{R}}\left(-\lambda_{E_{v}}^{T_{R}}\right) \\
\dot{\lambda}_{E_{i i i}}^{T_{R}} & =-\frac{1}{T_{A}}\left[K_{A}\left(\lambda_{E_{i v}}^{T_{R}}-\lambda_{E_{v}}^{T_{R}}\right)-\lambda_{E_{f d}}^{T_{R}}\right] \\
\dot{\lambda}_{E_{f d}}^{T_{R}} & =\frac{1}{T_{E}}\left[\lambda_{E_{i i i}}^{T_{R}}-\left(A e^{B E_{f d}} B \lambda_{E_{f d}}^{T_{R}}\right) E_{f d}-\left(A e^{B E_{f d}}+K_{E}\right) \lambda_{E_{f d}}^{T_{R}}\right] \\
\dot{\lambda}_{i v}^{T_{R}} & =\frac{1}{T_{R}}\left[K_{F} \dot{\lambda}_{E_{f d}}^{T_{R}}-\dot{\lambda}_{i v}^{T_{R}}\right]
\end{aligned}
$$

As condições iniciais em $t=t_{o}$ são $\lambda_{E_{v}}^{T_{R}}=\lambda_{E_{i i i}}^{T_{R}}=\lambda_{E_{f d}}^{T_{R}}=\lambda_{E_{i v}}^{T_{R}}=0$. 
Derivando o conjunto de equações 6.45 em relação a $T_{A}$

$$
\begin{aligned}
\dot{\lambda}_{E_{v}}^{T_{A}}= & \frac{1}{T_{R}}\left(-\lambda_{E_{v}}^{T_{A}}\right)=0 \\
\dot{\lambda}_{E_{i i i}}^{T_{R}}= & -\frac{1}{T_{A}^{2}}\left[K_{A}\left(V_{r e f}-E_{i v}-E v\right)-E_{i i i}\right]+ \\
& \frac{1}{T_{A}}\left[K_{A}\left(-\lambda_{E_{i v}}^{T_{A}}-\lambda_{E_{v}}^{T_{A}}\right)-\lambda_{E_{i i i}}^{T_{A}}\right] \\
\dot{\lambda}_{E_{f d}}^{T_{A}}= & \frac{1}{T_{E}}\left[\lambda_{E_{i i i}}^{T_{A}}-\left(A e^{B E_{f d}} B \lambda_{E_{f d}}^{T_{A}}\right) E_{f d}-\left(A e^{B E_{f d}}+K_{E}\right) \lambda_{E_{f d}}^{T_{A}}\right] \\
\dot{\lambda}_{i v}^{T_{A}}= & \frac{1}{T_{F}}\left[K_{F} \dot{\lambda}_{E_{f d}}^{T_{A}}-\dot{\lambda}_{i v}^{T_{A}}\right]
\end{aligned}
$$

As condições iniciais em $t=t_{o}$ são $\lambda_{E_{v}}^{T_{A}}=\lambda_{E_{i i i}}^{T_{A}}=\lambda_{E_{f d}}^{T_{A}}=\lambda_{E_{i v}}^{T_{A}}=0$.

Derivando o conjunto de equações 6.45) em relação a $K_{A}$

$$
\begin{aligned}
\dot{\lambda}_{E_{v}}^{K_{A}} & =\frac{1}{T_{R}}\left(-\lambda_{E_{v}}^{K_{A}}\right)=0 \\
\dot{\lambda}_{E_{i i i}}^{K_{A}} & =\frac{1}{T_{A}}\left[\left(V_{r e f}-E_{i v}-E v\right)-E_{i i i}+K_{A}\left(-\lambda_{E_{i v}}^{K_{A}}-\lambda_{E_{v}}^{K_{A}}\right)-\lambda_{E_{i i i}}^{K_{A}}\right] \\
\dot{\lambda}_{E_{f d}}^{K_{A}} & =\frac{1}{T_{E}}\left[\lambda_{E_{i i i}}^{T_{A}}-\left(A e^{B E_{f d}} B \lambda_{E_{f d}}^{K_{A}}\right) E_{f d}-\left(A e^{B E_{f d}}+K_{E}\right) \lambda_{E_{f d}}^{K_{A}}\right] \\
\dot{\lambda}_{E_{i v}}^{K_{A}} & =\frac{1}{T_{F}}\left[K_{F} \dot{\lambda}_{E_{f d}}^{K_{A}}-\lambda_{i v}^{T_{A}}\right]
\end{aligned}
$$

As condições iniciais em $t=t_{o}$ são $\lambda_{E_{v}}^{K_{A}}=\lambda_{E_{i i i}}^{K_{A}}=\lambda_{E_{f d}}^{K_{A}}=\lambda_{E_{i v}}^{K_{A}}=0$.

Derivando o conjunto de equações 6.45 em relação a $T_{E}$

$$
\begin{aligned}
\dot{\lambda}_{E_{v}}^{T_{E}}= & \frac{1}{T_{R}}\left(-\lambda_{E_{v}}^{T_{E}}\right)=0 \\
\dot{\lambda}_{E_{i i i}}^{T_{E}}= & \frac{1}{T_{A}}\left[K_{A}\left(-\lambda_{E_{i v}}^{T_{E}}-\lambda_{E_{v}}^{T_{E}}\right)-\lambda_{E_{i i i}}^{T_{E}}\right] \\
\dot{\lambda}_{E_{f d}}^{T_{E}}= & -\frac{1}{T_{E}^{2}}\left[E_{i i i}-\left(A e^{B E_{f d}}+K_{E}\right) E_{f d}\right]+ \\
& \frac{1}{T_{E}}\left[\lambda_{E_{i i i}}^{T_{E}}-\left(A e^{B E_{f d}} B \lambda_{E_{f d}}^{T_{E}}\right) E_{f d}-\left(A e^{B E_{f d}}+K_{E}\right) \lambda_{E_{f d}}^{T_{E}}\right] \\
\dot{\lambda}_{E_{i v}}^{T_{E}}= & \frac{1}{T_{F}}\left[K_{F} \dot{\lambda}_{E_{f d}}^{T_{E}}-\lambda_{E_{i v}}^{T_{E}}\right]
\end{aligned}
$$

As condições iniciais em $t=t_{o}$ são $\lambda_{E_{v}}^{T_{E}}=\lambda_{E_{i i i}}^{T_{E}}=\lambda_{E_{f d}}^{T_{E}}=\lambda_{E_{i v}}^{T_{E}}=0$. 
Derivando o conjunto de equações (6.45) em relação a $K_{E}$

$$
\begin{aligned}
\dot{\lambda}_{E_{v}}^{K_{E}} & =\frac{1}{T_{R}}\left(-\lambda_{E_{v}}^{K_{E}}\right)=0 \\
\dot{\lambda}_{E_{i i i}}^{K_{E}} & =\frac{1}{T_{A}}\left[K_{A}\left(-\lambda_{E_{i v}}^{K_{E}}-\lambda_{E_{v}}^{K_{E}}\right)-\lambda_{E_{i i i}}^{K_{E}}\right] \\
\dot{\lambda}_{E_{f d}}^{K_{E}} & =\frac{1}{T_{E}}\left[\lambda_{E_{i i i}}^{K_{E}}-\left(A e^{B E_{f d}} B \lambda_{E_{f d}}^{K_{E}}+1\right) E_{f d}-\left(A e^{B E_{f d}}+K_{E}\right) \lambda_{E_{f d}}^{K_{E}}\right] \\
\dot{\lambda}_{E_{i v}}^{K_{E}} & =\frac{1}{T_{F}}\left[K_{F} \dot{\lambda}_{E_{f d}}^{K_{E}}-\lambda_{E_{i v}}^{K_{E}}\right]
\end{aligned}
$$

As condições iniciais em $t=t_{o}$ são $\lambda_{E_{v}}^{K_{E}}=\lambda_{E_{i i i}}^{K_{E}}=\lambda_{E_{f d}}^{K_{E}}=\lambda_{E_{i v}}^{K_{E}}=0$.

Derivando o conjunto de equações (6.45) em relação a $A$

$$
\begin{aligned}
\dot{\lambda}_{E_{v}}^{A} & =\frac{1}{T_{R}}\left(-\lambda_{E_{v}}^{A}\right)=0 \\
\dot{\lambda}_{E_{i i i}}^{A} & =\frac{1}{T_{A}}\left[K_{A}\left(-\lambda_{E_{i v}}^{A}-\lambda_{E_{v}}^{A}\right)-\lambda_{E_{i i i}}^{A}\right] \\
\dot{\lambda}_{E_{f d}}^{A} & =\frac{1}{T_{E}}\left[\lambda_{E_{i i i}}^{A}-\left(A e^{B E_{f d}} B \lambda_{E_{f d}}^{A}\right) E_{f d}-\left(A e^{B E_{f d}}+K_{E}\right) \lambda_{E_{f d}}^{A}\right] \\
\dot{\lambda}_{E_{i v}}^{A} & =\frac{1}{T_{F}}\left[K_{F} \dot{\lambda}_{E_{f d}}^{A}-\lambda_{E_{i v}}^{A}\right]
\end{aligned}
$$

As condições iniciais em $t=t_{o}$ são $\lambda_{E_{v}}^{A}=\lambda_{E_{i i i}}^{A}=\lambda_{E_{f d}}^{A}=\lambda_{E_{i v}}^{A}=0$;

Derivando o conjunto de equações (6.45) em relação a $B$

$$
\begin{aligned}
\dot{\lambda}_{E_{v}}^{B} & =\frac{1}{T_{R}}\left(-\lambda_{E_{v}}^{B}\right)=0 \\
\dot{\lambda}_{E_{i i i}}^{B} & =\frac{1}{T_{A}}\left[K_{A}\left(-\lambda_{E_{i v}}^{B}-\lambda_{E_{v}}^{B}\right)-\lambda_{E_{i i i}}^{B}\right] \\
\dot{\lambda}_{E_{f d}}^{B} & =\frac{1}{T_{E}}\left[\lambda_{E_{i i i}}^{B}-\left(A e^{B E_{f d}} B \lambda_{E_{f d}}^{B}\right) E_{f d}-\left(A e^{B E_{f d}}+K_{E}\right) \lambda_{E_{f d}}^{B}\right] \\
\dot{\lambda}_{E_{i v}}^{B} & =\frac{1}{T_{F}}\left[K_{F} \dot{\lambda}_{E_{f d}}^{B}-\lambda_{E_{i v}}^{B}\right]
\end{aligned}
$$

As condições iniciais em $t=t_{o}$ são $\lambda_{E_{v}}^{B}=\lambda_{E_{i i i}}^{B}=\lambda_{E_{f d}}^{B}=\lambda_{E_{i v}}^{B}=0$.

Derivando o conjunto de equações (6.45) em relação a $T_{F}$

$$
\begin{aligned}
\dot{\lambda}_{E_{v}}^{T_{F}} & =\frac{1}{T_{R}}\left(-\lambda_{E_{v}}^{T_{F}}\right)=0 \\
\dot{\lambda}_{E_{i i i}}^{T_{F}} & =\frac{1}{T_{A}}\left[K_{A}\left(-\lambda_{E_{i v}}^{T_{F}}-\lambda_{E_{v}}^{T_{F}}\right)-\lambda_{E_{i i i}}^{T_{F}}\right] \\
\dot{\lambda}_{E_{f d}}^{T_{F}} & =\frac{1}{T_{E}}\left[\lambda_{E_{i i i}}^{T_{F}}-\left(A e^{B E_{f d}} B \lambda_{E_{f d}}^{T_{F}}\right) E_{f d}-\left(A e^{B E_{f d}}+K_{E}\right) \lambda_{E_{f d}}^{T_{F}}\right] \\
\dot{\lambda}_{E_{i v}}^{T_{F}} & =-\frac{1}{T_{F}^{2}}\left[K_{F} E f d-E_{i v} r\right]+\frac{1}{T_{F}}\left[K_{F} \lambda_{E_{f d}}^{T_{F}}-\lambda_{E_{i v}}^{T_{F}}\right]
\end{aligned}
$$


As condições iniciais em $t=t_{o}$ são $\lambda_{E_{v}}^{T_{F}}=\lambda_{E_{i i i}}^{T_{F}}=\lambda_{E_{f d}}^{T_{F}}=\lambda_{E_{i v}}^{T_{F}}=0$.

Derivando o conjunto de equações 6.45 em relação a $K_{F}$

$$
\begin{aligned}
\dot{\lambda}_{K_{v}}^{K_{F}} & =\frac{1}{T_{R}}\left(-\lambda_{E_{v}}^{K_{F}}\right)=0 \\
\dot{\lambda}_{E_{i i i}}^{K_{F}} & =\frac{1}{T_{A}}\left[K_{A}\left(-\lambda_{E_{i v}}^{K_{F}}-\lambda_{E_{v}}^{K_{F}}\right)-\lambda_{E_{i i i}}^{K_{F}}\right] \\
\dot{\lambda}_{E_{f d}}^{T_{F}} & =\frac{1}{T_{E}}\left[\lambda_{E_{i i i}}^{K_{F}}-\left(A e^{B E_{f d}} B \lambda_{E_{f d}}^{K_{F}}\right) E_{f d}-\left(A e^{B E_{f d}}+K_{E}\right) \lambda_{E_{f d}}^{K_{F}}\right] \\
\dot{\lambda}_{E_{i v}}^{T_{F}} & =-\frac{1}{T_{F}^{2}}\left[E f d+K_{F} \dot{\lambda}_{E_{f d}}^{K_{F}}-\dot{\lambda}_{E_{i v}}^{K_{F}}\right]
\end{aligned}
$$

As condições iniciais em $t=t_{o}$ são $\lambda_{E_{v}}^{K_{F}}=\lambda_{E_{i i i}}^{K_{F}}=\lambda_{E_{f d}}^{K_{F}}=\lambda_{E_{i v}}^{K_{F}}=0$.

A variável de entrada é a tensão terminal da máquina síncrona e a variável de saída é a tensão de campo do sistema de excitação. Isto é mostrado na figura (6.35).

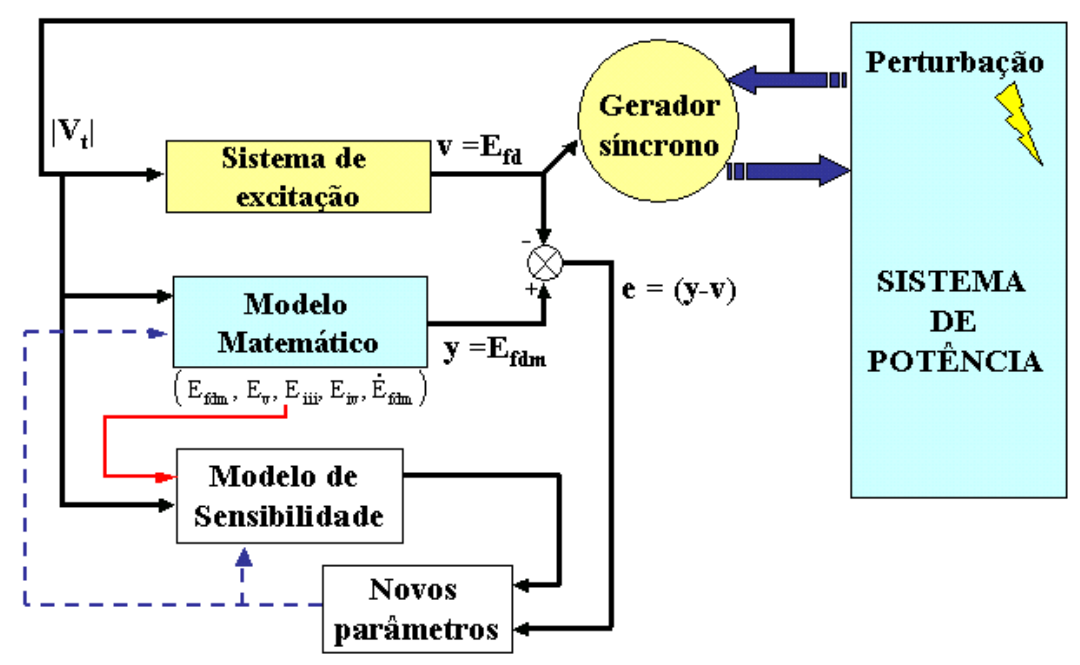

Figura 6.35: Diagrama esquemático para estimação dos parâmetros do sistema de excitação DC1.

Aplicando uma perturbação no sistema de potência com as mesmas considerações feitas no modelo anterior, a metodologia identificou satisfatoriamente os 9 parâmetros deste modelo. Também foram feitos outros testes com estimativas iniciais dos parâmetros $+/-20 \%$ dos valores verdadeiros em outras direções com resultados satisfatórios. Entretanto para estimativas iniciais mais distantes a 20\% o procedimento diverge. 
Na figura 6.36 mostra-se a tensão de campo antes e depois o processo de estimação, e na tabela 6.13 apresenta-se o resumo do processo de estimação. Os parâmetros foram estimados com excelente precisão.
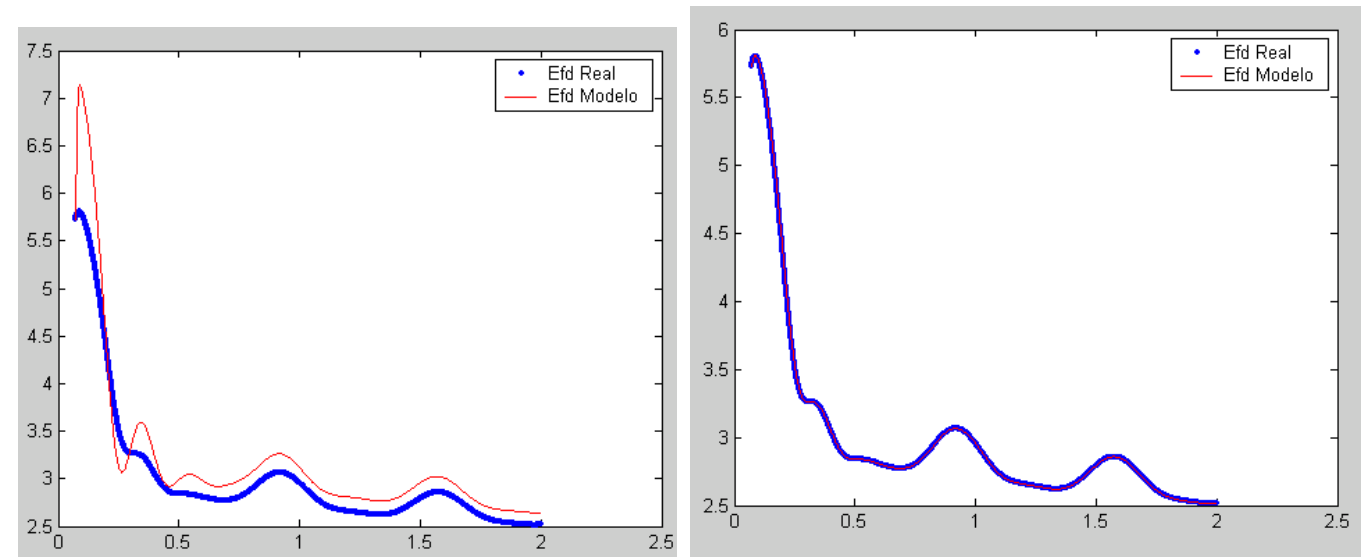

Figura 6.36: Tensão de campo antes e depois a estimação dos parâmetros

Tabela 6.13: Parâmetros do sistema de excitação DC1.

\begin{tabular}{|c|c|c|c|c|c|c|c|c|c|}
\hline Iter & $T_{R}$ & $T_{A}$ & $K_{A}$ & $T_{E}$ & $K_{E}$ & $\mathrm{~A}$ & $\mathrm{~B}$ & $T_{F}$ & $K_{F}$ \\
\hline Inicial & 0.0400 & 0.0720 & 40.00 & 0.0416 & -0.0462 & 0.0010 & 1.011 & 0.800 & 0.0666 \\
\hline 1 & 0.0496 & 0.0642 & 62.70 & 0.0643 & -0.0674 & 0.0015 & 1.176 & 0.918 & 0.0759 \\
\hline 2 & 0.0499 & 0.0601 & 54.02 & 0.0569 & -0.0397 & 0.0014 & 1.244 & 0.994 & 0.0828 \\
\hline 3 & 0.0500 & 0.0600 & 49.94 & 0.0518 & -0.0389 & 0.0012 & 1.262 & 0.999 & 0.0832 \\
\hline Final & 0.0500 & 0.0600 & 50.00 & 0.0520 & -0.0386 & 0.0012 & 1.264 & 1.000 & 0.0832 \\
\hline Verd. & 0.0500 & 0.0600 & 50.00 & 0.0520 & -0.0385 & 0.0012 & 1.264 & 1.000 & 0.0832 \\
\hline Erro(\%) & 0.0 & 0.0 & 0.00 & 0 & 0.259 & 0.0 & 0.0 & 0.00 & 0.0 \\
\hline
\end{tabular}

Neste caso, mostrou-se a possibilidade da estimativa de parâmetros 'on-line'. 


\section{Capítulo 7}

\section{Conclusões}

No presente trabalho foi apresentada a metodologia de sensibilidade de trajetória para a determinação de parâmetros do gerador síncrono e do sistema de excitação.

As principais contribuições deste trabalho foram: a avaliação do potencial desta técnica para a estimação de parâmetros do gerador síncrono e do sistema de excitação a partir de dados obtidos em testes com o gerador em operação (teste 'on-line') e a aplicação com sucesso da técnica de sincronização no processo de estimação de parâmetros.

A metodologia estima os parâmetros a partir dos dados da perturbação medidos com o gerador conectado à rede. Os medidores são instalados e ficam aguardando a ocorrência de uma perturbação externa suficientemente grande para excitar as variáveis dinâmicas do sistema cujos parâmetros desejam-se estimar. Uma vez detectada a perturbação, os dados referentes a um certo período de tempo são armazenados para serem então processados pela metodologia de sensibilidade de trajetória.

Também foram detalhadas as dificuldades, encontradas na aplicação da metodologia baseada na técnica de sensibilidade da trajetória para a identificação de parâmetros. Entre eles tem-se:

1. Estimação simultânea de vários parâmetros: alguns pesquisadores relataram dificuldades para estimar simultaneamente vários parâmetros da máquina síncrona. Em (Sanchez et al., 1988), por exemplo, estas dificuldades sur- 
giram no momento de identificar simultaneamente os parâmetros do eixo direto e eixo em quadratura da máquina síncrona, sendo necessário fixar alguns dos parâmetros para serem determinados por outras técnicas. Se o parâmetro fixado não for o suficientemente preciso, o erro do parâmetro fixo pode acarretar em estimativas erradas.

2. Indisponibilidade de medidas: algumas equações diferenciais (utilizadas no modelo matemático) incluem medidas indisponíveis ou difíceis de serem medidas (por exemplo, medidas de fluxo).

3. Indisponibilidade de condições iniciais de equações diferenciais: existem equações diferenciais com condições diferenciais com condições iniciais que dependem dos parâmetros a serem estimados. Desta forma, a metodologia não pode ser aplicada.

4. Falta de Robustez da metodologia em relação as estimativas iniciais dos parâmetros: se os valores iniciais dos parâmetros a estimar não estiverem próximos dos valores verdadeiros a metodologia não consegue estimar os parâmetros (os parâmetros divergem).

Para contornar estas dificuldades foram propostas e estudadas as seguintes alternativas :

1. A estimação por etapas pode ser utilizada como alternativa para contornar o problema de estimação simultânea de vários parâmetros, isto é, partindo de modelos simples a modelos mais detalhados. Isto foi realizado para o modelo clássico e o modelo de 'um eixo'. Esta idéia ainda precisa ser trabalhada para ser aplicada em modelos mais detalhados.

Outra alternativa para solucionar este problema é a análise das escolha das entradas e saídas. Na seção 6.3 apresenta-se vários testes considerando diferentes entradas e saídas para contornar este problema.

2. O problema de indisponibilidade de medidas pode ser resolvido transformando as equações originais por outras com variáveis mensuráveis. Esta 
proposta foi aplicada satisfatoriamente ao modelo clássico da máquina síncrona, porém, é necessário um estudo mais aprofundado para aplicar esta idéias a modelos mais detalhados.

3. O problema de indisponibilidade das condições iniciais das equações diferenciais pode ser resolvido considerando-as como parâmetros estimados simultaneamente com os outros parâmetros da máquina síncrona. Isto foi aplicado no caso do modelo de 'um eixo' e do modelo de 'dois eixos' da máquina síncrona. No caso do modelo de 'um eixo', é necessário estimar o valor inicial da tensão interna de eixo em quadratura $E_{q o}^{\prime}$ e no modelo de 'dois eixos' além deste valor é necessário estimar a condição inicial da tensão interna de eixo direto $E_{d o}^{\prime}{ }^{1}$.

4. Para melhorar a robustez da metodologia, em relação a valores iniciais dos parâmetros, foi utilizada a técnica de sincronização de sistemas no processo de estimação. Isto foi aplicado com sucesso para a estimação dos parâmetros da máquina síncrona. A técnica de sincronização , permitiu uma maior faixa de incerteza de todos os parâmetros. Mais estudos são necessários para explorar as vantagens desta técnica quando aplicadas no processo de estimação, sendo portanto um tema para futuras pesquisas.

Além disso avaliou-se também a potencialidade da metodologia para a identificação dos parâmetros mecânicos da máquina síncrona (constante de inércia H, e constante de amortecimento D). Tais parâmetros foram estimados de forma independente da identificação dos outros parâmetros da máquina síncrona. Neste caso a metodologia mostrou-se bem robusta em relação as estimativas iniciais dos parâmetros permitindo incertezas de até $+/-70 \%$ em relação aos valores verdadeiros.

A metodologia foi utilizada para estimar os parâmetros do sistema de excitação de dois tipos padronizados pelo IEEE (tipo ST1A e DC1). Em ambos os

\footnotetext{
${ }^{1}$ As tensões $E_{d o}^{\prime}$ e $E_{q o}^{\prime}$ são proporcionais aos fluxos segundo o eixo direto e quadratura
} 
casos a metodologia estimou com sucesso os parâmetros desses modelos. No caso do sistema de excitação ST1A os parâmetros foram identificados com um erro inferior a 2.6\%, e no caso do sistema de excitação DC1 a metodologia conseguiu identificar os 9 parâmetros deste modelo simultaneamente, mostrando a potencialidade desta técnica, com um erro inferior a $0.26 \%$. Mostrou-se claramente a possibilidade de estimativa de parâmetros a partir de dados de perturbações obtidos com o gerador conectado à rede.

Por fim, analisou-se a influência dos parâmetros da máquina síncrona e seu sistema de excitação na estabilidade transitória. Para tal objetivo foram realizados testes de estabilidade em um pequeno sistema de potência sujeito a incertezas nos parâmetros, destacando a importância da correta identificação dos parâmetros (vide apêndice $\mathrm{A}$ ).

Espera-se que o trabalho apresentado possa contribuir na aplicação de identificação de parâmetros da máquina síncrona e dos controladores associados. Muitas idéias surgiram ao longo da presente pesquisa para aperfeiçoar a metodologia de sensibilidade de trajetória, no sentido de viabilizar a sua aplicação em sistemas reais a partir de dados obtidos de perturbações com o gerador conectado à rede. Dentre as recomendações para melhorar a metodologia podem-se citar:

1. Explorar a utilização da teoria de sincronização na identificação de parâmetros.

2. Aplicar as transformações, como aquela realizada na representação clássica da máquina síncrona, para modelos mais detalhados.

3. Realizar uma análise de outros testes para a identificação de parâmetros como, por exemplo, aplicação de perturbações no sistema de excitação ou no circuito de campo.

4. Realizar a identificação de parâmetros de um sistema de pequeno porte para validar a metodologia proposta.

5. Aplicar a metodologia em um sistema de grande porte. 


\section{Bibliografia}

Amano, M., Watanabe, M. e Banjo, M. (1999). Self testing and self tuning of power system and stabilizers using prony analysis, Power Engineering Society 1999 Winter Meeting 1: 655-660.

Anderson, P. M. e Fouad, A. (1993). Power System Control and Stability, IEEE Press.

Benchluch, S. e Chow, J. H. (1993). A trajectory sensitivity method for the identification of nonlinear excitation system models, IEEE Transaction on Energy Conversion 8: 159-164.

Bollinger, K. E., Norum, E. e Gu, W. (1991). A device for testing power system stabilizer parameters on-line, Proceedings of the 33rd Midwest Symposium on Circuits and Systems 1: 374-376.

Bortoni, E. C. (2001). Modelagem de máquinas síncronas de pólos salientes empregando técnicas de resposta em frequência, Tese de Doutorado, Universidade de São Paulo, Escola Politécnica, Biblioteca Ep, EPEL /FT-1239.

Bortoni, E. C. e Jardini, J. A. (2002). Identification of synchronous machine parameters using load rejection test data, IEEE Transactions on Energy Conversion 17: 242-247.

Cari, E. T. e Alberto, L. F. (2003). Relatório de atividade programada: Estudo de metodologias para a estimação de parâmetros em geradores síncronos, Technical report, Universidade de São Paulo EESC Engenharia ELétrica. 
Chow, J. H., Glinkowski, M., Murphy, R. J., Cease, T. W. e Kosaka, N. (1999). Generator and exciter parameter estimation of fort patrick henry hydro unit 1, IEEE Transactions on Energy Conversion 14: 923-929.

Cruz, J. B. (1972). Feedback System, McGraw-Hill.

Dandeno, P. L., C., K. H., C., A., I., K., Salon, S. J. e Saunder, R. M. (1999). Experience with standstill frequency response (ssfr) testing and analysis of salient pole synchronous machines, IEEE Transactions on Energy Conversion 14: 1209-1217.

de Mello, F. P. e Hannett, F. P. (1998). Determination of synchronous machine stator and field leakage inductances from standstill frequency response tests, IEEE Transactions on Power Systems 3: 1625-1632.

Demitry, K. (2004). Hydro turbine-governor model validation in pacific northwest, Transaction on Power on Energy Conversion 19: 1144-1149.

Feltes, J. W., F., O. B., E., U., S., Z. e Abi, S. N. (2002). Deriving model parameter from field test measurement, IEEE Computer Application in Power Systems 15: 30-36.

Gameiro, M. e Rodrigues, H. M. (2000). Applications of robust synchronization to communication systems, Applicable Analysis $\mathbf{7 9}$.

Guo, T. Y., Liu, C., Chen, Y. T., Ko, C. e Huan, C. T. (1995). Identification of model parameters of excitation system and power system stabilizer of mingtan 6 via finalization field tests, IEEE Transaction on Power System 10: 795-802.

Hannett, L. N. e Feltes, J. W. (1993). Derivation of generator excitation system and turbine governor parameter frond test, Coloquium of CIGRE study committee 38. Florianópolis Brasil . 
Henschel, S. e Dommel, H. W. (1999). Noniterative synchronous machine standstill frequency-response test data analysis, IEEE Transactions on Energy Conversion 14: 553-560.

Hiskens, I. A. e Koeman (1998). Parameter estimation from power system disturbance measurements, International Conference on Energy Management and Power Delivery 2: 667-672.

Hiskens, I. A. e Pai, M. A. (2000). Trajectory sensitivity analysis of hybrid systems, IEEE Transactions on Fundamental Theory and Applications 47: 204220.

Huang, D. e Guo, R. (2004). Identifying parameter by identical synchronization between different systems, Chaos 14.

IEEE (1981). Excitation system models for power system stability studies, Technical report, Working Group on Computer Modelling of Excitation Systems.

IEEE (1986). Standard definitions for excitation systems for synchronous machines, Technical report, Std 421.1-1986, Power Engineering Society.

IEEE (1992). Recommended practice for excitation system models for power system stability studies, Technical report, Std 421.5-1992, Power Engineering Society.

IEEE (1995). Guide test procedures for synchronous machines, Technical report, Std 115-1995, Power Engineering Society.

IEEE (2002). Guide for synchronous generator modeling practices and applications in power system stability analyses, Technical report, Std 1110-2002, Power Engineering Society.

Karayaka, H. B. (2003). Synchronous generator model identification and parameter estimation from operation data, IEEE Transactions on Energy Conversion 18: 121-126. 
Karrari, M. e P., M. O. (2004). Identification of physical parameter of a synchronous generator from online measurements, IEEE Transactions on Energy Conversion pp. 1-9.

Kim, J., Moon, S. e Lee, J. (2001a). A new optimal avr parameter tuning method using on-line performance indices of frequency-domain, IEEE Power Engineering Society Summer Meeting 3: 1554-1559.

Kim, J., Moon, S. e Lee, J. (2001b). A new optimal avr parameter tuning method using on line performance indices of frequency domain, Power Engineering Society Summer Meeting 3: 1554-1559.

Kimbark, E. W. (1995). Power System Stability, Vol. 3, IEEE Press.

Kundur, P. (1994). Power System Stability and Control, Mc Graw Hill.

Kyriakides, E., Heydt, G. T. e Vittal, V. (2004). On line estimation of synchronous generator parameter using current observer and graphics user interface, Transaction on Power on Energy Conversion 19.

Le, L. X. e Wilson, W. (1998). Synchronous machine parameter identification: A time domain approach, IEEE Transaction on Power System 3: 241-248.

Liu, C. S., Hsu, Y. Y., Jeng, L. H., Lin, C. J., Huang, C. T., Liu, A. e Li, T. H. (1993). Identification of exciter constants using a coherence function based weighted least squares approach, IEEE Transaction on Power System 8: 460-467.

Melgoza, J. J., Heydt, G. T., Keyhany, A., Agrawal, B. L. e Selin, D. (2001). Synchronous machine parameter estimation using the hartley series, Transaction on Energy Conversion 10: 49-54.

Mello, F. P. (1994). Measuremnt of synchronous machine rotor angle from analysis of zero sequence harmonic components of machine terminal voltage, IEEE Transactions on Power Delivery 9: 1770-1776. 
Murdoch, A., Sanderson, H. C. e Lawson, R. A. (2000). Excitation systemsperformance specification to meet interconnection requirements, Power Engineeing Society Winter Meeting 1: 597-602.

Oliveira, R. V. (2003). Análise da coordenação entre estabilizadores clássicos e robustos em sistemas elétricos de potência, Tese de Mestrado, Universidade de São Paulo, Escola de Engenharia de São Carlos, Biblioteca Central, TESE-EESC O48ar.

Ong, C. M. (1998). Dynamic Simulation of Electric Machinery Using Matlab/Simulink, Prentice Hall Ptr.

Pecora, L. M. e Caroll, T. (1990). Synchronization in chaotic systems, Physical review letters 60: 821-824.

Rodrigo, A. R., Alberto, L. F. e Bretas, N. G. (2000). Modelagem de máquinas síncronas aplicada ao estudo de estabilidade de sistemas elétricos de potência, Technical report, Universidade de São Paulo EESC Engenharia ELétrica.

Rodrigues, H. M. (1996). Abstract methods for synchronization and applications, Applicable Analysis 62.

Sanchez, G. J., Bridenbaugh, C. J., Bowler, C. e Edmonds, J. (1988). Trajectory sensitivity based identification of synchronous generator and excitation system parameters, IEEE Transaction on Power System 3: 1814-1822.

Saunders, R. M. (1991). Syncrhonous machine standstill frequency response test data analysis, IEEE Transactions on Energy Conversion 6.

Shen, S., Zhu, S. e Han, B. (1991). Identification of parameters of synchronous machine and excitation system by online test, Advances in Power System Control, Operation and Management 2: 716-719. 
Shouzhen, Z., Shande, S. e Houlian, C. (1993). Effects of the excitation system parameters on power system transient stability studies, Advances in Power System Control, Operation and Management 1: 532-535.

Soliman, S. A. e Alkandari, A. (1996). Optimal parameter estimation of synchronous machines from the digitized sudden short-circuit armature current, IEEE AFRICON 4th $\mathbf{1 .}$

Tomovic, R. (1963). Sensitivity Analysis of Dynamic System, McGraw-Hill.

Touhami, O., Guesbaoni, H. e Iung, C. (1994). Synchronous machine parameter identification by a multitime scale technique, IEEE Transactions on Industry Applications 30.

Tso, S. K. e Cheung, S. P. (1994). Consideration of excitation control for transient stability assessment, Proccedings of the Third IEEE Conference on Control Application 1: 485-490.

Vas, P. (1993). Parameter Estimation, COndition Monitoring, and Diagnosis of Electrical Machines, Clarendon Press Oxford.

Vermeulen, H., Strauss, J. M. e J.M., S. (2002). Online estimation of synchronous generator parameters using prbs perturbations, IEEE Transactions on Power System 17: 694-700.

Weeber, K. (1997). Determination of dynamic parameter of large hydrogenerators by finite element simulation of three phase sudden short circuit tests, Electric Machines and Drives Conference Record 10.

Zali, S. M., Ariffin, A. E., Mohamed, A. e A., H. (2000). Implementation of synchronous machine parameter derivation in matlab, IEEE TENCON 2000. Proceedings 1: 218-223.

Zhao, Z., Zheng, F., Gao, J. e Xu, L. (1995). A dinamic on-line parameter identification and full-scale system experimental verfication for large synchronous machines, IEEE Transactions on Energy Conversion 3: 392-398. 
Zhengming, Z., Fungshi, Z., Jide, G. e Longya, X. (1995). A dinamic on-line parameter identification and full-scale system experimental verification for large synchronous machines, Transaction on Power on Energy Conversion 10: 392-398. 


\section{Apêndice A}

\section{Influência dos parâmetros da} máquina síncrona e do sistema de excitação na estabilidade

\section{transitória}

\section{A.1 Introdução}

Neste capítulo são realizadas simulações computacionais para estudar a influência dos parâmetros da máquina e do sistema de excitação na estabilidade transitória. Como se relatou no capítulo 1, por diversas razões, verifica-se muita incerteza em relação ao conhecimento dos parâmetros tanto do gerador síncrono como do sistema de excitação. Estas incertezas surgem devido à perda dos dados, reajuste do ganho de algum componente não documentado, variação de parâmetros devido ao envelhecimento de componentes do sistema, etc. Na perda de dados de algum dos dispositivos é comum utilizar os parâmetros de outros dispositivos similares. Tudo isto faz com que as simulação computacionais sejam diferentes daquilo que acontece no sistema real, e em muitos casos estas diferenças podem ser significativas podendo inclusive não detectar instabilidade no sistema.

Um exemplo onde estas incertezas ocorrem é no sistema elétrico do Peru. 
Após a interligação do sistema Centro-Norte com o sistema Sul em Setembro de 2000 apareceram muitos problemas relacionados com a estabilidade do sistema. Vários estudos foram feitos para determinar a margem de estabilidade, porém não se tem tanta confiabilidade nos resultados devido as incertezas nos parâmetros daquele sistema. No evento acontecido em nove de Junho de 2002 1 durante um teste de estabilidade (inclusão de uma pequena perturbação) após o desligamento da linha de transmissão L-1011 (vide figura A.1) o sistema apresentou oscilações inter-áreas trazendo como conseqüência o desligamento das linhas L-1005 e L-1006 e interrupção do fornecimento da energia em muitas áreas do sistema. Tais acontecimentos não puderam ser previstos através das simulações computacionais. A principal razão porque isto acontece, de acordo com o COES (comitê de operação econômica do sistema), é devido à não disponibilidade dos dados corretos de algumas unidades geradoras.

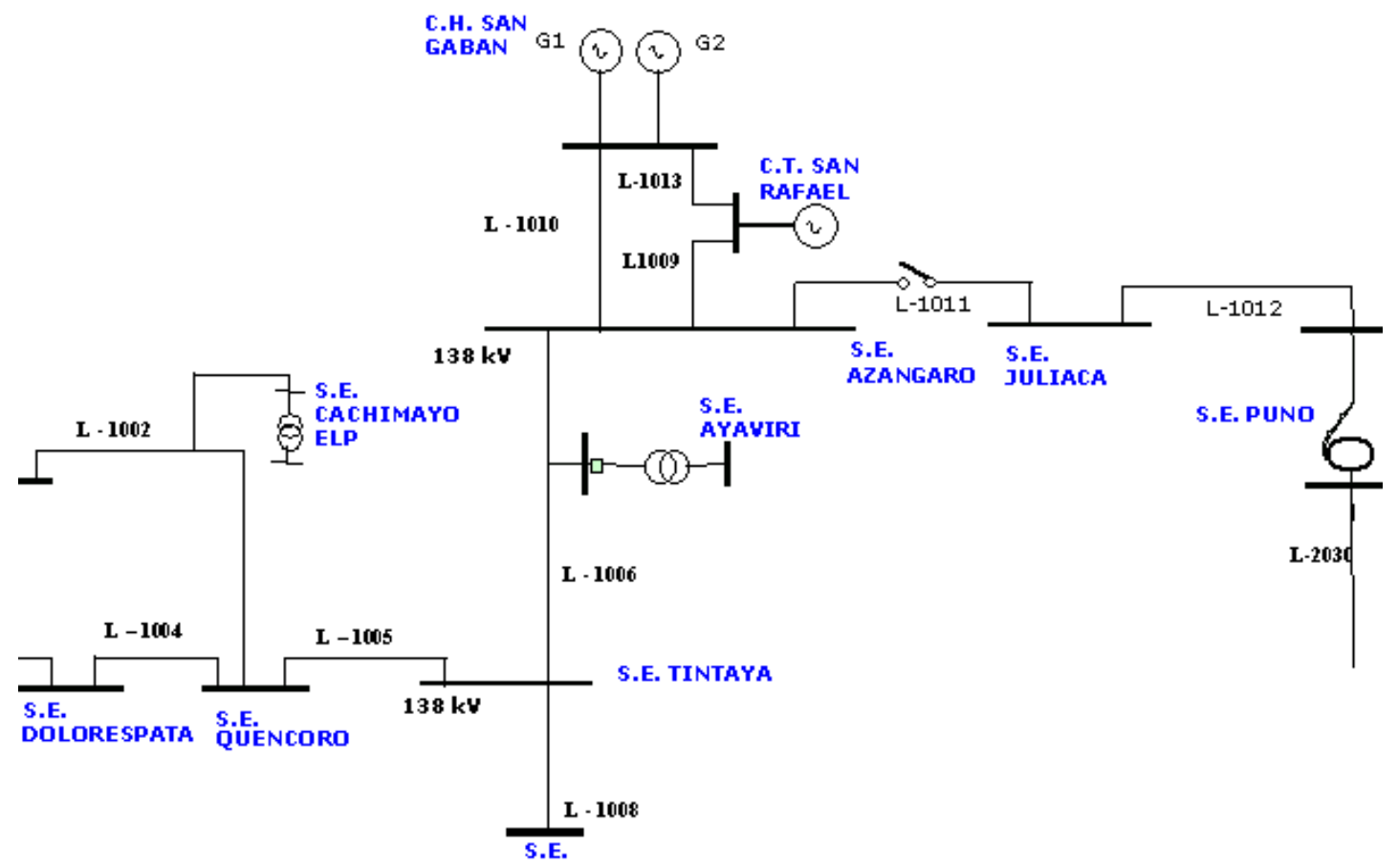

Figura A.1: Parte do Sistema Elétrico Sul do Peru.

Uma das alternativas para resolver este problema é a realização de testes em

\footnotetext{
${ }^{1}$ dados obtidos da página web do COES-PERÚ, www.coes.org.pe
} 
cada gerador e então obter os parâmetros corretos. O grande inconveniente é os testes devem ser realizados com a unidade desligada do sistema.

Outros problemas por incertezas nos parâmetros foram relatados em (Demitry, 2004). O WECC (Western Electricity Coordinating Council) 10 de agosto de 1996 relatou um colapso de tensão enquanto as simulações computacionais prediziam uma operação normal (sistema estável) nas mesmas condições. Na figura A.2 mostra-se a comparação entre as curvas de potência de transferência real (registrada) e a obtida computacionalmente (interligação Califórnia-Oregon). Após tal evento a política aprovada pelo WSCC é que todos os geradores com potência superior a 10MW deveriam ser testados e os seus modelos validados a cada cinco anos.

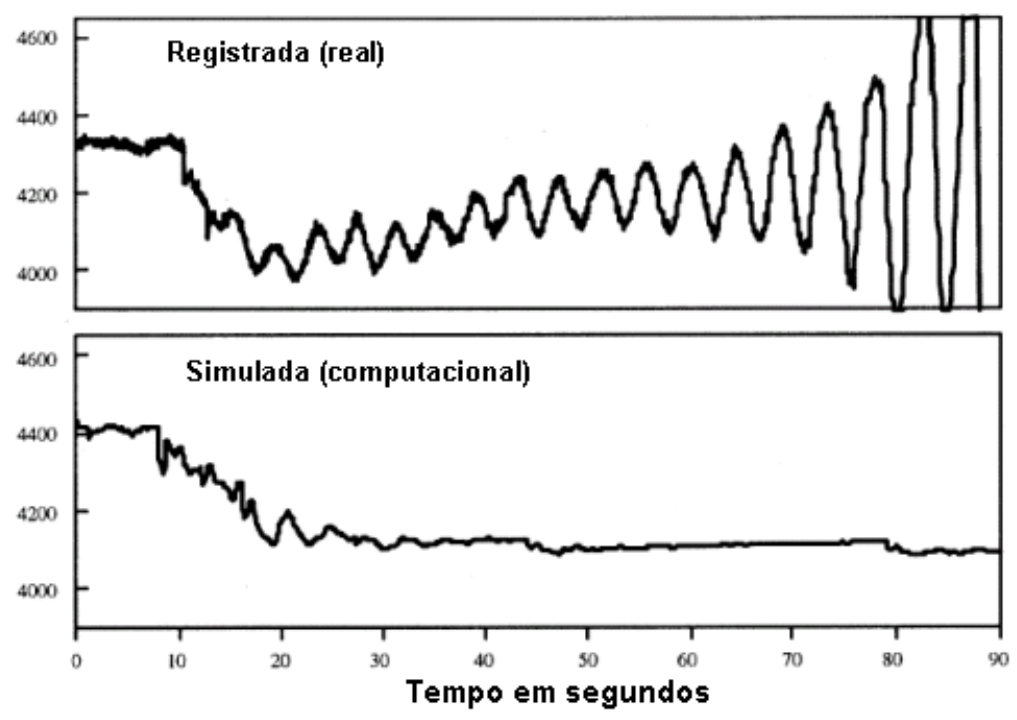

Figura A.2: Comparação entre a potências de transferência registrada e a simulada computacionalmente.

Estes exemplos mostram a grande importância de conhecer os parâmetros, da máquina síncrona e do sistema de excitação, corretamente identificados. 


\section{A.2 Testes Realizados}

Nesta seção apresentam-se vários testes com o objetivo de estudar a influência dos parâmetros na estabilidade transitória. Para isto, foram realizadas várias simulações em um sistema de potência na presença de uma falta trifásica. As simulações são realizadas com os valores verdadeiros dos parâmetros e considerando que existem incertezas em alguns dos parâmetros do gerador em estudo. Posteriormente são comparados os tempos críticos de abertura do sistema com parâmetros verdadeiros e com parâmetros com incertezas.

O sistema de potência no qual realizam-se as simulações é mostrado na figura A.3 (sistema similar apresentado no livro (Kundur, 1994)). Este sistema possui duas áreas, quatro geradores, quatro transformadores e onze barras. O gerador três é considerado como referência na simulação (barramento infinito). Os geradores G1, G2 e G4 são modeladas através do modelo de 'dois eixos' e possuem o mesmo sistema de excitação ST1A definido no capítulo quatro. Além disso G2 e G3 possuem PSS. As simulações são realizadas no programa NEPLAN2 versão demo 52.

Os dados utilizados para a simulação estão em p.u. com potência base de $S_{B}=900$ MVA e com tensão base de $230 \mathrm{kV}$ no lado de alta e $20 \mathrm{kV}$ no lado de baixa tensão.

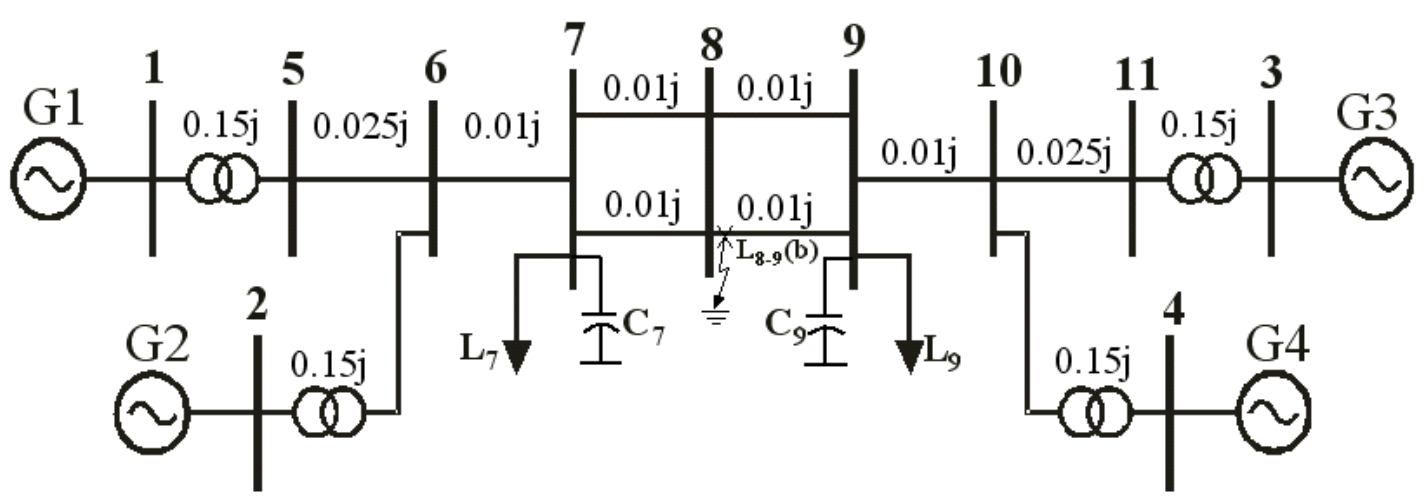

Figura A.3: Sistema de potência para a aplicação do testes de estabilidade transitória

Na tabela A.1 apresenta-se as tensões na barras de geração em regime per-

\footnotetext{
${ }^{2} \mathrm{O}$ programa está disponível no site http://www.neplan.com
} 
manente (pré-falta). A barra $n^{\circ} 3$ é considerado como barra 'slack' no cálculo de fluxo de carga e como barramento infinito no cálculo de estabilidade transitória.

Tabela A.1: Tensões nas barras terminais dos geradores na condição pré-falta.

\begin{tabular}{|c|c|c|c|c|}
\hline Barra & Tipo & $\begin{array}{c}\text { Potência Ativa } \\
\text { (p.u.) }\end{array}$ & $\begin{array}{c}\text { Potência Reativa } \\
\text { (p.u.) }\end{array}$ & $\begin{array}{c}\text { Tensão } \\
\text { (p.u.) }\end{array}$ \\
\hline 1 & PV & 0.777 & 0.206 & $1.03 \angle-0.590$ \\
\hline 2 & PV & 0.777 & 0.206 & $1.03 \angle-1.637$ \\
\hline 3 & 'slack' & 0.798 & 0.195 & $1.03 \angle 0.00$ \\
\hline 4 & PV & 0.777 & 0.224 & $1.01 \angle-2.635$ \\
\hline
\end{tabular}

Os dados de carga são mostradas na figura A.2 e os parâmetros dos geradores G1, G2, G4 são mostrados na tabela A.3.

Tabela A.2: Dados das cargas do sistema de potência.

\begin{tabular}{|c|c|c|c|}
\hline Barra & $P_{L}$ (p.u.) & $Q_{L}$ (p.u.) & $Q_{C}$ (p.u.) \\
\hline 7 & 1.074 & 0.111 & 0.388 \\
\hline 9 & 1.956 & 0.111 & 0.388 \\
\hline
\end{tabular}

Tabela A.3: Valores dos parâmetros dos geradores.

\begin{tabular}{|c|c|c|c|c|c|c|c|c|c|}
\hline Gerador & $x_{d}$ & $x_{q}$ & $x_{d}^{\prime}$ & $x_{q}^{\prime}$ & $x_{l}$ & $T_{d o}^{\prime}$ & $T_{q o}^{\prime}$ & $\mathrm{H}$ & $\mathrm{D}$ \\
\hline G1,G2,G4 & 1.81 & 1.76 & 0.30 & 0.65 & 0.15 & 8 & 1 & 3.5 & 2.2 \\
\hline
\end{tabular}

Os dados do sistema de excitação são apresentados na tabela A.4 e os parâmetros dos PSS's na tabela A.5 (o esquema do PSS pode ser obtido de (Kundur, 1994)).

Tabela A.4: Valores dos parâmetros do sistema de excitação.

\begin{tabular}{|c|c|c|c|c|}
\hline Gerador & Tipo & $T_{R}$ & $K_{A}$ & $V_{\text {ref }}$ \\
\hline G1 & ST1A & 0.05 & 200 & 1.03 \\
\hline G2 & ST1A & 0.01 & 200 & 1.01 \\
\hline G4 & ST1A & 0.01 & 200 & 1.01 \\
\hline
\end{tabular}


Tabela A.5: Parâmetros do PSS.

\begin{tabular}{|c|c|c|c|c|c|c|c|c|c|}
\hline & \multirow{2}{*}{ Gerador } & & & Ganho & \multicolumn{3}{|c|}{ washout } & \multicolumn{4}{|c|}{ blocos avanço-atraso } \\
\cline { 4 - 10 } & $f_{r e f}$ & $K_{p u}$ & $K_{S T A B}$ & $K_{w}$ & $T_{w}$ & $T_{1 N}$ & $T_{1 D}$ & $T_{2 N}$ & $T_{2 D}$ \\
\hline G2, G4 & 60 & $\frac{1}{60}$ & 20 & 1 & 10 & 0.02 & 0.05 & 5.4 & 3 \\
\hline
\end{tabular}

O SEP é testado aplicando-se uma falta trifásica na linha $L_{8-9}(b)$ (vide figura A.3 próximo a barra 8. A falta é eliminada pelo acionamento dos dispositivos de proteção isolando a linha em curto em um tempo de $t=0.07 \mathrm{~s}$. Estima-se o tempo crítico de abertura dos relés para que o sistema seja estável em tais condições.

Inicialmente calcular-se-á o tempo crítico do SEP com os parâmetros verdadeiros em seguida o mesmo é feito alterando os parâmetros do gerado G1.

\section{A.2.1 Sistema de Potência com parâmetros verdadeiros}

O SEP da figura A.3 é testado com a perturbação especificada na seção anterior e com os parâmetros verdadeiros.

Simulando o teste, observa-se que o sistema é estável com um tempo crítico de $t=0.094 \mathrm{~s}$ (figura A.4(a)) e é instável com um tempo $t=0.095 \mathrm{~s}$. (figura A.4(a))

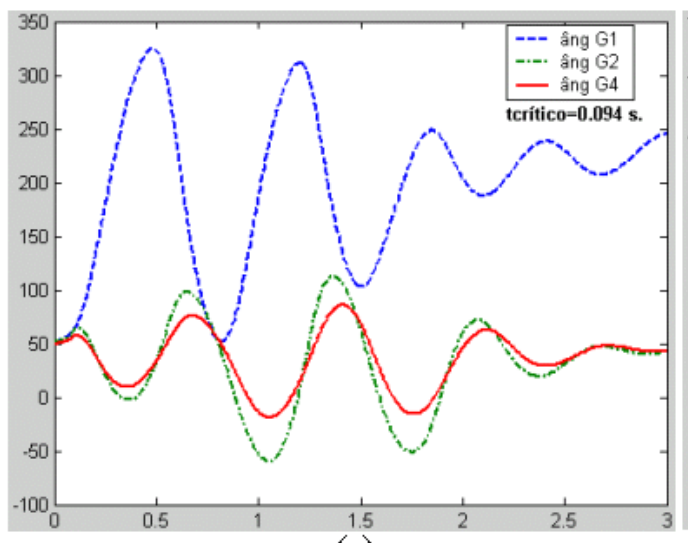

(a)

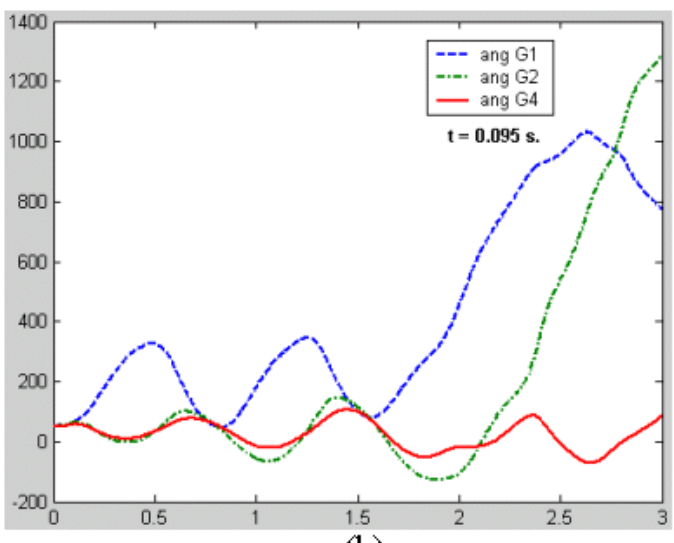

(b)

Figura A.4: Resposta do ângulo do rotor dos geradores com parâmetros verdadeiros 


\section{A.2.2 Influência dos parâmetros do sistema de excitação}

Neste teste o SEP da figura A.3 é testado considerando a mesma perturbação especificada anteriormente. Nestes caso, as simulações são realizadas considerando incertezas nos parâmetros do sistema de excitação do gerador G1. Os valores dos parâmetros de $K_{A}$ e de $T_{R}$ são alterados em +/- 20\% de seus valores verdadeiros em diferentes direções. O tempo crítico de abertura do relé para a eliminação da falta é determinado para vários casos e são mostrados na tabela A.6.

Tabela A.6: Influência dos parâmetros do sistema de excitação ST1A do Gerador G1 no cálculo do tempo crítico.

\begin{tabular}{|c|c|c|c|}
\hline Caso & Parâmetros perturbados & Tcrítico & Variação (\%) \\
\hline 1 & $K_{A} \cdot 1.2=240$ & 0.081 & 13.83 \\
\hline 2 & $K_{A} \cdot 0.8=160$ & 0.112 & -19.14 \\
\hline 3 & $T_{R} \cdot 1.2=0.06$ & 0.100 & -6.38 \\
\hline 4 & $T_{R} \cdot 0.8=0.04$ & 0.089 & 5.31 \\
\hline & $K_{A} \cdot 1.2=240$ & & \\
5 & $T_{R} \cdot 1.2=0.06$ & 0.085 & 9.57 \\
\hline 6 & $K_{A} \cdot 1.2=240$ & & \\
\hline & $T_{R} \cdot 0.8=0.04$ & 0.074 & 21.27 \\
\hline 7 & $K_{A} \cdot 0.8=160$ & 0.093 & \\
\hline & $T_{R} \cdot 0.8=0.04$ & 0.106 & -12.7 \\
\hline 8 & $K_{A} \cdot 0.8=160$ & & \\
\hline
\end{tabular}

Como se observa na tabela A.6 na maior diferença no cálculo do tempo crítico do sistema com incerteza acontece quando $K_{A}$ é considerado em -20\% e $T_{R}$ em $+20 \%$ de seus valores verdadeiros.

\section{A.2.3 Influência dos parâmetros elétricos da máquina síncrona}

Neste caso, as simulações são realizadas considerando incerteza nos parâmetros elétricos do gerador G1. Os parâmetros são alterados em +/- 20\% de seus valores 
verdadeiros em diferentes direções. As alterações nos parâmetros são realizadas respeitando que $x_{d}>x_{q}>x_{q}^{\prime}>x_{d}^{\prime}$. O tempo crítico de abertura do relé para a eliminação da falta é determinado e são mostrados na tabela A.7.

Tabela A.7: Influência dos parâmetros elétricos da máquina síncrona no tempo crítico de abertura.

\begin{tabular}{|c|c|c|c|}
\hline Caso & Parâmetros perturbados & Tcrítico & Variação (\%) \\
\hline 1 & $x_{d} \cdot 1.2=2.172$ & 0.094 & 0 \\
\hline 2 & $x_{q} \cdot 0.8=1.408$ & 0.089 & -4.26 \\
\hline 3 & $x_{q}^{\prime} \cdot 1.2=0.78$ & 0.090 & 4.25 \\
\hline 4 & $x_{q}^{\prime} \cdot 0.8=0.52$ & 0.098 & -4.25 \\
\hline 5 & $x_{d}^{\prime} \cdot 1.2=0.36$ & 0.094 & 0.00 \\
\hline 6 & $x_{d}^{\prime} \cdot 0.8=0.0 .24$ & 0.094 & 0.00 \\
\hline 7 & $T_{d o}^{\prime} \cdot 1.2=9.6$ & 0.107 & -13.82 \\
\hline 8 & $T_{d o}^{\prime} \cdot 0.8=6.4$ & 0.078 & 17.02 \\
\hline 9 & $T_{q o}^{\prime} \cdot 1.2=1.2$ & 0.093 & 1.06 \\
\hline 10 & $T_{q o}^{\prime} \cdot 0.8=0.8$ & 0.094 & 0 \\
\hline & $x_{q} \cdot 0.8, x_{q}^{\prime} \cdot 1.2$ & & \\
\hline 11 & $T_{d o}^{\prime} \cdot 0.8, T_{q o}^{\prime} \cdot 1.2$ & 0.070 & 25.53 \\
\hline
\end{tabular}

Segundo a tabela A.7, os parâmetros mais influentes no cálculo do tempo crítico são $T^{\prime} d o$ e $x_{q}^{\prime}$. Uma variação de $-20 \%$ do valor real de $T_{d o}^{\prime}$ implica em uma variação de aproximadamente $17 \%$ no tempo crítico de abertura. Na mesma tabela também é mostrado que, alterando vários parâmetros (caso $\left.n^{\circ} 11\right)$, o tempo crítico de abertura pode diferir até em $25 \%$ do valor verdadeiro. O tempo crítico permaneceu praticamente insensível à alteração dos valores dos parâmetro $x_{d}^{\prime} \mathrm{e}$ $T_{q o}^{\prime}$. 


\section{A.2.4 Influência dos parâmetros mecânicos da máquina síncrona}

Neste caso as simulações são realizadas considerando incerteza nos parâmetros mecânicos (H e D) do gerador G1. Os parâmetros são alterados em +/- 20\% de seus valores verdadeiros em diferentes direções. O tempo crítico de abertura do relé para a eliminação da falta é determinado e são mostrados na tabela A.8.

Tabela A.8: Influência dos parâmetros mecânicos da máquina síncrona no tempo crítico de abertura.

\begin{tabular}{|c|c|c|c|}
\hline Caso & Parâmetros perturbados & Tcrítico & Variação (\%) \\
\hline 1 & $H \cdot 1.2=4.2$ & 0.106 & -12.76 \\
\hline 2 & $H \cdot 0.8=2.8$ & 0.083 & 11.70 \\
\hline 3 & $D \cdot 1.2=0.78$ & 0.095 & -1.06 \\
\hline 4 & $D \cdot 0.8=2.64$ & 0.093 & 1.06 \\
\hline & $H \cdot 0.8=2.64$ & & \\
5 & $D \cdot 0.8=0.78$ & 0.081 & 13.83 \\
\hline
\end{tabular}

Neste caso o parâmetro que mais influência tem na determinação do tempo crítico de abertura é a constante de inércia H. Uma alteração de $-20 \%$ do seu valor verdadeiro produz uma variação do tempo crítico de abertura de $11.7 \%$. A influência da constante de amortecimento D é menor no cálculo do tempo crítico de abertura.

Nos casos analisados anteriormente viu-se a importância da estimação dos parâmetros tanto da máquina síncrona como do sistema de excitação na estabilidade transitória. Destaca-se também que apenas os parâmetros do gerador G1 foram alterados e que mudando os parâmetros dos outros geradores a influência pode ser maior. 


\section{Apêndice B}

\section{Publicações Originadas desta}

\section{Pesquisa}

- E.P.T. Cari, L.F.C. Alberto, A. C. P. Martins, N. G. Bretas "Methodology for the parameter estimation of synchronous generator and excitation system." ACEITO para apresentação no IEEE St.Petersburg Power Tech Conference, que será realizado de 27 a 30 de junho de 2005, em St. Petersburg, Rusia. 
